\title{
Emotion and cognition in Parkinson's disease
}

Citation for published version (APA):

Moonen, A. J. H. (2016). Emotion and cognition in Parkinson's disease: etiology and neurobiological mechanisms. [Doctoral Thesis, Maastricht University]. https://doi.org/10.26481/dis.20160324am

Document status and date:

Published: 01/01/2016

DOI:

10.26481/dis.20160324am

Document Version:

Publisher's PDF, also known as Version of record

\section{Please check the document version of this publication:}

- A submitted manuscript is the version of the article upon submission and before peer-review. There can be important differences between the submitted version and the official published version of record.

People interested in the research are advised to contact the author for the final version of the publication, or visit the DOI to the publisher's website.

- The final author version and the galley proof are versions of the publication after peer review.

- The final published version features the final layout of the paper including the volume, issue and page numbers.

Link to publication

\footnotetext{
General rights rights.

- You may freely distribute the URL identifying the publication in the public portal. please follow below link for the End User Agreement:

www.umlib.nl/taverne-license

Take down policy

If you believe that this document breaches copyright please contact us at:

repository@maastrichtuniversity.nl

providing details and we will investigate your claim.
}

Copyright and moral rights for the publications made accessible in the public portal are retained by the authors and/or other copyright owners and it is a condition of accessing publications that users recognise and abide by the legal requirements associated with these

- Users may download and print one copy of any publication from the public portal for the purpose of private study or research.

- You may not further distribute the material or use it for any profit-making activity or commercial gain

If the publication is distributed under the terms of Article $25 \mathrm{fa}$ of the Dutch Copyright Act, indicated by the "Taverne" license above, 


\section{EMOTION AND COGNITION IN PARKINSON’S DISEASE}

ETIOLOGY AND NEUROBIOLOGICAL MECHANISMS 
(C) Anja J.H. Moonen, Maastricht 2016

No part of this book may be reproduced or transmitted in any form or by any means, without prior permission in writing by the author, or when appropriate, by the publishers of the publication.

Cover design: $\quad$ studio sanne dijkstra, met dank aan Shelley Verhoef

Layout: $\quad$ Anja Moonen, Mark Proosten

Printing: Gildeprint

Publisher: $\quad$ NeuroPsych Publishers

ISBN: $\quad$ 978-94-6233-223-2 


\title{
EMOTION AND COGNITION IN PARKINSON’S DISEASE
}

\author{
ETIOLOGY AND NEUROBIOLOGICAL MECHANISMS
}

\author{
ProefschrifT \\ Ter verkrijging van de graad van doctor aan de Universiteit Maastricht, \\ op gezag van de Rector Magnificus, Prof. dr. Luc Soete, \\ volgens het besluit van het College van Decanen, \\ in het openbaar te verdedigen op donderdag \\ 24 maart 2016 om 16:00uur \\ door
}

Anja Julia Hendrik Moonen

Geboren op 27 mei 1987 te Geleen 


\section{Promotor}

Prof. dr. F.R.J. Verhey

\section{Co-promotor}

Dr. A.F.G. Leentjens

\section{Beoordelingscommissie}

Prof. dr. R.W.H.M. Ponds (voorzitter)

Prof. dr. I.Y.R. Myin-Germeys (Universiteit van Leuven, België)

Prof. dr. R.J. van Oostenbrugge (Maastricht UMC+)

Prof. dr. Y. Temel (Maastricht UMC+)

Prof. dr. O.A van den Heuvel (VU Medisch Centrum)

The research described in this thesis was performed at the School for Mental Health and Neuroscience, Department of Psychiatry and Neuropsychology, Maastricht University, the Netherlands. Part of the research described in Chapter 3 was performed at the Institute for Neuroscience and Medicine (INM-3), Research Centre Jülich, Germany. The research presented in Chapter 4 and 5 was carried out in collaboration with the Department of Neurology and Movement Disorders, University of Lille, France.

Stichting Parkinson Fonds sponsored the research described in Chapter 3 and the Michael J. Fox Foundation for Parkinson's research sponsored the research presented in Chapter 4 and 5.

Printing of this thesis was kindly supported by Lundbeck.

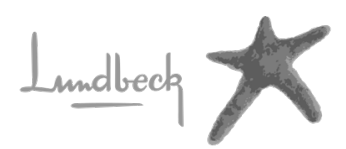


Voor jou, klein wonder 



\section{CONTENTS}

ChAPTER 1 General Introduction

ChAPTER 2 Neurobiological correlates of emotional processing in

Parkinson's disease: a systematic review of experimental studies

Chapter 3 An fMRI study into the neurobiology of emotional processing in Parkinson's disease: does increased medial prefrontal cortex activation compensate for striatal dysfunction?

Chapter 4 Cognitive disorders in Parkinson's disease: confirmation of a spectrum of severity

(Parkinsonism and Related Disorders, 2015)

ChAPTER 5 Reduced grey matter density associated with early cognitive decline in Parkinson's disease: a voxel-based morphometry study

Chapter 6 Modeling depression in Parkinson's disease: disease-specific and non-specific risk factors

(Neurology, 2013)

ChAPTER 7 Severity of depression and anxiety are predictors of response to antidepressant treatment in Parkinson's disease

(Parkinsonism and Related Disorders, 2014)

Chapter 8 General Discussion

Summary

Samenvatting

Knowledge valorization

Dankwoord/Acknowledgments

Curriculum Vitae

Publications

Thesis defenses from MHeNS - School for Mental Health and Neuroscience 



\section{ChAPTER 1}

GenerAL INTRODUCTION 


\section{PARKINSON'S DISEASE}

Parkinson's disease (PD) is one of the most prevalent neurodegenerative disorders among the elderly population. The estimated prevalence of PD is approximately $1 \%$ among people of 65 years or older and increases with age. ${ }^{1}$ According to the Queens Square Brain Bank criteria, a clinical diagnosis of PD requires the presence of the core feature 'bradykinesia' together with at least one of the following motor symptoms: resting tremor, rigidity, or postural instability. ${ }^{2}$ However, in over $20 \%$ of PD patients the non-motor symptoms form the clinical picture of the disease. ${ }^{3}$ Examples of non-motor symptoms are neuropsychiatric disorders, sleep disorders, autonomic symptoms, and sensory symptoms (see Table 1).,5

Table 1. Non-motor symptoms in PD and their underlying neural substrates ${ }^{*}$

\begin{tabular}{ll}
\hline Non-motor symptoms in PD & Presumed underlying brain structures \\
\hline $\begin{array}{l}\text { Olfactory loss impairments in odor } \\
\text { detection, identification and discrimination }\end{array}$ & $\begin{array}{l}\text { Olfactory bulb, anterior olfactory nucleus, } \\
\text { amygdala, perirhinal cortex }\end{array}$ \\
$\begin{array}{l}\text { Dysautonomia gastrointestinal } \\
\text { disturbances, urinary dysfunction, sexual } \\
\text { dysfunction, orthostatic hypotension }\end{array}$ & $\begin{array}{l}\text { Amygdala, dorsal IX/X motor complex, } \\
\text { intermediolateral column of the spinal cord, } \\
\text { sympathetic ganglia, enteric and autonomic } \\
\text { plexuses }\end{array}$ \\
$\begin{array}{l}\text { Mood disorders/emotional dysfunction } \\
\text { depression, anxiety }\end{array}$ & $\begin{array}{l}\text { Locus coeruleus, raphe nuclei, amygdala, } \\
\text { mesolimbic, mesocortical cortex }\end{array}$ \\
$\begin{array}{l}\text { Sleep disorders REM behavior disorder, } \\
\text { excessive daytime sleepiness, insomnia }\end{array}$ & $\begin{array}{l}\text { Nucleus subcoeruleus, pedunculopontine } \\
\text { nucleus, thalamus, hypothalamus }\end{array}$ \\
$\begin{array}{l}\text { Hallucinations, psychosis } \\
\text { Other non-motor symptoms pain, } \\
\text { apathy, fatigue, mid-life obesity, impaired } \\
\text { color discrimination, restless legs } \\
\text { syndrome, rhinorrhea }\end{array}$ & Amygdala, limbic cortex \\
\hline
\end{tabular}

Although many non-motor symptoms emerge in advanced stages of the disease, some symptoms such as olfactory dysfunction, Rapid Eye Movement (REM) sleep behaviour disorder (RBD), cognitive impairment, and depression can be present already in early stages of PD or even years before the diagnosis. ${ }^{6}$ However, regardless of which disease stage, non-motor symptoms severely affect the quality of life of PD patients and their caregivers and have a negative impact on the prognosis of the disease. ${ }^{7-10}$ Both motor and non-motor symptoms are differently represented 
among PD patients and can change along the course of the disease progression, making PD an extremely complex illness. Moreover, due to existing overlap with motor symptoms, some of the non-motor symptoms are still under-recognized and consequently undertreated. ${ }^{11}$

\section{Neuropathology of PD}

The clinical motor symptoms of PD present themselves only when $40-50 \%$ of dopaminergic neurons in the substantia nigra pars compacta (SNpc) have been lost leading to a massive dopaminergic denervation and depletion. ${ }^{12-14}$ Together with the formation of Lewy neurites and intraneuronal Lewy bodies, which mainly consist of aggregations of the misfolded protein alpha-synuclein, the progressive loss of dopaminergic neurons in the SNpc is considered as the pathological hallmark of $\mathrm{PD} .{ }^{15}$ Concurrent with damage to the SNpc, the ventral tegmental area and subregions of the amygdala become affected, followed by damage in the hippocampus and parts of the neocortex (i.e., prefrontal cortex and higher association areas). ${ }^{13}$

However, the staging procedure of PD pathology as proposed by Braak and colleagues ${ }^{13}$ suggests that even before damage to the SNpc becomes apparent and before PD is diagnosed, Lewy body pathology is usually already present in the dorsal IX/X motor complex in the brainstem, the olfactory bulb, the locus coeruleus, and the caudal raphe nuclei. ${ }^{16}$ These phenomena fit well with the fact that several 'non-motor symptoms', such as olfactory dysfunction, REM sleep behavior disorder, autonomic dysfunction, and visual dysfunction often precede the clinical diagnose of PD. The proposed Lewy body pathology by Braak and colleagues does, however, not explain the presence of other preclinical symptoms, such as restless legs, or the presence of other non-motor symptoms such as cognitive impairment, depression, and hallucinations, as these symptoms are more closely associated with neural degeneration rather than Lewy body distribution., ${ }^{4,17}$

As such, the underlying neurobiological mechanisms of many non-motor symptoms still remain unclear. Several neuroimaging studies in PD have highlighted the role of dopamine in non-motor symptoms, ${ }^{18,19}$ yet the serotonergic, noradrenergic, and cholinergic neurotransmitter systems appear to be involved as well. ${ }^{20,21}$ According to the inflammatory hypothesis, the release of pro-inflammatory cytokines through activated microglia in the brain can initiate neurotransmissive 
alterations in these systems similar to those seen in affective disorders such as depression. $^{22,23}$

With this thesis we aim to expand our understanding of neurobiological mechanisms for at least a subgroup of non-motor symptoms in PD, namely emotional disturbances and cognitive symptoms.

\section{NEUROPSYCHIATRY IN PD}

Non-motor symptoms of PD are now more and more recognized as being an integral part of the disease, with a significant impact on the quality of life of patients and their caregivers, leading to greater health care expenses. ${ }^{24,25}$ This thesis focuses on a subgroup of non-motor symptoms that are particularly disabling for patients: neuropsychiatric symptoms. The majority of PD patients experience one or more of these non-motor symptoms during the course of the disease. ${ }^{26}$ Most prevalent are anxiety (40-50\%), depression (20-40\%), dementia (20-30\%), hallucinations (20-40\%), apathy (17-40\%), and impulse control disorders (36-60\%). ${ }^{11,27}$ High comorbidity exists among these disorders, making it increasingly difficult to recognize them. ${ }^{28}$ This thesis will be mainly focused on emotional disturbances and cognitive disorders.

Although the impact of these disorders on a patient's health status has been widely recognized, treatments are still scarce and non-optimal. The efficacy of pharmacological treatment for depression has been demonstrated in multiple clinical trials, although this may be limited to PD patients with moderate-to-severe depression. ${ }^{29}$ Non-pharmacological treatments such as Cognitive Behavioral Therapy (CBT) have been shown to be more promising for milder cases of depression, given that the intervention is tailored to the needs and capabilities of PD patients. ${ }^{30-32}$ The beneficial effects for anxiety were less clear, possibly because most treatments are mainly focused on depression and consider anxiety as a comorbid symptom of depression rather than a separate disorder. So far, there have been no pharmacological nor psychotherapeutic trials that specifically aim at treating anxiety disorders in PD. Apathy also remains a challenging disorder to treat. Although a dopaminergic basis involving depleted limbic areas has been suggested, ${ }^{33}$ apathy can be unresponsive to dopaminergic therapy. Furthermore, masking of apathy by depression and the lack of motivation may further complicate treatment. ${ }^{34-36}$ Dopaminergic treatment that targets motor symptoms in PD may improve some cognitive deficits, yet prolonged use of levodopa or the use of specific dopamine- 
agonists can have detrimental effects on cognition. ${ }^{37}$ Cholinergic treatment of cognitive deficits is available, but more appropriate for early stages of PD dementia. ${ }^{38}$

The search for effective treatments is still ongoing. However, in order to develop effective interventions, both pharmacological and psychotherapeutic, it is essential that we first unravel the underlying neurobiological mechanisms of neuropsychiatric disorders in PD. With respect to anxiety, depression, and apathy, an overlying deficit in emotional processing may provide a neural basis for these disorders. A large network of subcortical limbic areas and cortical frontal areas appear to be involved and a disbalance in this circuit may lead to disturbances in emotion regulation (see Chapter 2 for an extensive literature review). For cognition, we see a large spectrum of cognitive disorders where PD patients with different cognitive phenotypes show specific cognitive deficits. ${ }^{39}$ By exploring the neural correlates of these cognitive phenotypes we may be able to promptly identify patients who are at risk for developing more severe cognitive decline and PD dementia. As such, more specific and patient tailored pharmacotherapy and cognitive training options can be developed and provided in early stages of cognitive decline.

\section{AIMS OF THIS THESIS}

The main aim of this thesis was to explore the neurobiological mechanisms and correlates of emotional disorders and cognitive decline in PD. For this we used blood-oxygen-level-dependent functional magnetic resonance imaging (BOLD-fMRI) to investigate emotional processing in PD patients and healthy controls (Chapter 3) and we used analytic tools and structural MRI in a large sample of PD patients for respectively the identification of cognitive phenotypes (Chapter 4) and the their anatomical correlates (Chapter 5). A secondary aim was to identify risk factors for developing depression in PD (Chapter 6) and to search for specific conditions that determine response to treatment of depression with antidepressants (Chapter 7) by using advanced statistical approaches. Within these chapters, the following research questions will be addressed:

1. Which neurobiological mechanisms underlie emotional processing deficits in PD?

2. How can we explain intact emotional processing at the behavioral level in PD patients despite deficient neural activation patterns in striatal and limbic areas involved in emotional processing? 
3. Which cognitive phenotypes can be distinguished in PD and to what extent can neuroanatomical markers predict early cognitive decline?

4. What is the relative contribution of disease-specific and non-specific markers for developing depression in PD?

5. Which factors can predict improvement in depressive symptoms during antidepressant treatment in PD patients?

\section{OUTLINE OF THIS THESIS}

Chapter 2 provides a systematic review on the neurobiology of emotional processing deficits in PD. By including only experimental studies that assessed both behavioral and neurobiological aspects of emotional processing, we aimed at increasing our understanding of the pathophysiology of this non-motor aspect of PD. We have further tried to put our results in the broader context of a functional-anatomic model of affective neurocircuitry, and highlighted the role of dopamine within this circuitry. PRISMA guidelines were followed, including an extensive quality assessment of included papers.

In Chapter 3 we cross-sectionally investigated implicit emotional processing and its functional correlates in PD patients and matched healthy control subjects using BOLD-fMRI. Previous studies report an intact ability in PD patients to explicitly recognize and categorize emotions, despite neurobiological alterations, such as reduced grey matter volume ${ }^{40,41}$ or region-specific alterations of activity seen on electroencephalogram $(E E G)^{42-45}$ or fMRI. ${ }^{18,19,46,47}$ Especially regions within ventral frontal-subcortical limbic circuitry including the amygdala, the orbitofrontal cortex (OFC), the ventral anterior cingulate cortex (ACC), the ventral striatum, and the ventrolateral prefrontal cortex (VIPFC) appear to be affected in PD. For a correct function of these structures intact dopaminergic projections are required (Moonen et al. submitted). However, the reported intact emotional recognition abilities despite neural abnormalities in the ventral part of emotional neurocircuitry, suggests the involvement of compensational neural mechanisms or the use of behavioral strategies in PD patients.

In Chapter 4 we aimed to confirm prospectively a previously performed exploratory cluster analysis based on retrospective neuropsychological data that identified five cognitive phenotypes, ranging from cognitively intact PD patients with intact performance in every cognitive domain to very severely impaired patients, with 
a progressive gradient. ${ }^{39}$ Validation of the obtained classification would be essential for exploring the anatomical and functional correlates of these clusters. Chapter 5 is therefore based on the results from Chapter 4 as our aim was here to identify the neuroanatomical correlates of each of the cognitive phenotypes using structural MRI. More specifically, we were looking for regional differences in grey matter atrophy between these clusters. Considering the progressive gradient we observed for cognitive performance, we expected to see a similar progression of grey matter loss when comparing the healthy cluster to the other clusters. We also hoped to identify specific markers of each group as well as clinical risk factors for developing dementia in PD.

Chapter 6 provides a model for depression in PD in which we compare the relative contribution of $\mathrm{PD}$-specific and nonspecific risk factors to this model. Existing cross-sectional studies have largely focused on PD-specific risk factors for depression, such as more severe motor symptoms, longer disease duration, more advanced disease stage, greater limitations in disease-related activities of daily living $(A D L)$, higher daily levodopa equivalent dose, and the presence of other non-motor symptoms such as hallucinations, sleep disturbances, and dysautonomia. ${ }^{48-51}$ However, few studies have examined the role of general risk factors for depression in PD, such as gender, age, level of education, cognitive impairment, and physical disease.

In Chapter 7 we aimed to identify factors that predict improvement in depressive symptoms during antidepressant treatment in depressed PD patients. Although many PD patients receive antidepressants, in only half of them the treatment appeared to effective. ${ }^{52}$ Recently, the randomized controlled Study of Antidepressants in PD (SAD-PD) demonstrated the effectiveness of paroxetine (a selective serotonin reuptake inhibitor) and venlafaxine extended release (a selective serotonin and norepinephrine reuptake inhibitor) in the active treatment of depressive syndromes in PD patients as compared to a placebo treatment. ${ }^{53}$ Identifying factors that predict response during antidepressant treatment of PD patients may have important implications for the treatment of depression in this population.

Chapter 8 summarizes and discusses the main findings of all previous chapters in the light of current scientific research. Clinical implications and recommendations for future research are provided. 


\section{REFERENCES}

1. Wirdefeldt K, Adami H-O, Cole P, Trichopoulos D, Mandel J. Epidemiology and Etiology of Parkinson's Disease: A Review of the Evidence. European journal of epidemiology 2011;26:1-58.

2. Hughes AJ, Daniel SE, Kilford L, Lees AJ. Accuracy of Clinical Diagnosis of Idiopathic Parkinson's Disease: A Clinico-Pathological Study of 100 Cases. J Neurol, Neurosur Ps 1992;55:181-184.

3. Pfeiffer RF. Non-Motor Symptoms in Parkinson's Disease. Parkinsonism Relat Disord 2016;22:S119-S122.

4. Chaudhuri KR, Healy DG, Schapira AH. Non-Motor Symptoms of Parkinson's Disease: Diagnosis and Management. The Lancet Neurology 2006;5:235-245.

5. Tolosa E, Gaig C, Santamaría J, Compta Y. Diagnosis and the Premotor Phase of Parkinson Disease. Neurology 2009;72:S12-S20.

6. Chaudhuri KR, Naidu Y. Early Parkinson's Disease and Non-Motor Issues. Journal of neurology 2008;255:33-38.

7. Martinez-Martin P, Rodriguez-Blazquez C, Kurtis MM, Chaudhuri K. The Impact of Non-Motor Symptoms on Health-Related Quality of Life of Patients with Parkinson's Disease. Mov Disord 2011;26:399-406.

8. Schrag A. Quality of Life and Depression in Parkinson's Disease. J Neurol Sci 2006;248:151-157.

9. Slawek J, Derejko M, Lass P. Factors Affecting the Quality of Life of Patients with Idiopathic Parkinson's Disease-a Cross-Sectional Study in an Outpatient Clinic Attendees. Parkinsonism Relat Disord 2005;11:465-468.

10. Weintraub D, Moberg PJ, Duda JE, Katz IR, Stern MB. Effect of Psychiatric and Other Nonmotor Symptoms on Disability in Parkinson's Disease. Journal of the American Geriatrics Society 2004;52:784-788.

11. Aarsland D, Kramberger M. Neuropsychiatric Symptoms in Parkinson's Disease. Journal of Parkinson's Disease 2015;5:659-667.

12. Ferrer I. Early Involvement of the Cerebral Cortex in Parkinson's Disease: Convergence of Multiple Metabolic Defects. Progress in neurobiology 2009;88:89-103.

13. Braak H, Ghebremedhin E, Rüb $U$, Bratzke $H$, Del Tredici K. Stages in the Development of Parkinson's Disease-Related Pathology. Cell Tissue Res 2004;318:121-134.

14. Fearnley JM, Lees AJ. Ageing and Parkinson's Disease: Substantia Nigra Regional Selectivity. Brain 1991;114:2283-2301.

15. Spillantini MG, Schmidt ML, Lee VM-Y, Trojanowski JQ, Jakes R, Goedert M. ASynuclein in Lewy Bodies. Nature 1997;388:839-840.

16. Del Tredici K, Rüb U, de Vos RA, Bohl JR, Braak H. Where Does Parkinson Disease Pathology Begin in the Brain? J Neuropath Exp Neur 2002;61:413-426.

17. Jellinger KA. A Critical Reappraisal of Current Staging of Lewy-Related Pathology in Human Brain. Acta neuropathologica 2008;116:1-16.

18. Tessitore A, Hariri AR, Fera F, Smith WG, Chase TN, Hyde TM, et al. Dopamine Modulates the Response of the Human Amygdala: A Study in Parkinson's Disease. J Neurosci 2002;22:9099-9103.

19. Delaveau P, Salgado-Pineda P, Fossati P, Witjas T, Azulay JP, Blin O. Dopaminergic Modulation of the Default Mode Network in Parkinson's Disease. Eur 
Neuropsychopharm 2010;20:784-792.

20. Remy P, Doder M, Lees A, Turjanski N, Brooks D. Depression in Parkinson's Disease: Loss of Dopamine and Noradrenaline Innervation in the Limbic System. Brain 2005;128:1314-1322.

21. Barone P. Neurotransmission in Parkinson's Disease: Beyond Dopamine. European Journal of Neurology 2010;17:364-376.

22. Pessoa Rocha N, Reis HJ, Vanden Berghe P, Cirillo C. Depression and Cognitive Impairment in Parkinson's Disease: A Role for Inflammation and Immunomodulation? Neuroimmunomodulation 2014;21:88-94.

23. Leentjens AF. Parkinson Disease: Depression-Risk Factor or Early Symptom in Parkinson Disease? Nat Rev Neurol 2015;11:432-433.

24. Schenkman M, Zhu CW, Cutson TM, Whetten-Goldstein K. Longitudinal Evaluation of Economic and Physical Impact of Parkinson's Disease. Parkinsonism Relat D 2001;8:41-50.

25. Weintraub D. Neuropsychiatric Symptoms in Parkinson Disease and Dementia with Lewy Bodies: What Geriatric Psychiatry Can Learn. The American Journal of Geriatric Psychiatry 2013;21:497-500.

26. Aarsland D, Larsen JP, Lim NG, Janvin C, Karlsen K, Tandberg E, et al. Range of Neuropsychiatric Disturbances in Patients with Parkinson's Disease. Journal of Neurology, Neurosurgery \& Psychiatry 1999;67:492-496.

27. Papapetropoulos S, Mash D. Psychotic Symptoms in Parkinson's Disease. Journal of neurology 2005;252:753-764.

28. Shulman LM, Taback RL, Bean J, Weiner WJ. Comorbidity of the Nonmotor Symptoms of Parkinson's Disease. Movement Disorders 2001;16:507-510.

29. Schrag A, Sauerbier A, Chaudhuri KR. New Clinical Trials for Nonmotor Manifestations of Parkinson's Disease. Movement Disorders 2015;30:1490-1504.

30. Dobkin RD, Menza M, Allen LA, Gara MA, Mark MH, Tiu J, et al. Cognitive-Behavioral Therapy for Depression in Parkinson's Disease: A Randomized, Controlled Trial. American Journal of Psychiatry 2011;168:1066-1074.

31. Troeung L, Egan SJ, Gasson N. A Waitlist-Controlled Trial of Group Cognitive Behavioural Therapy for Depression and Anxiety in Parkinson's Disease. BMC psychiatry 2014;14:19.

32. Calleo JS, Amspoker AB, Sarwar AI, Kunik ME, Jankovic J, Marsh L, et al. A Pilot Study of a Cognitive-Behavioral Treatment for Anxiety and Depression in Patients with Parkinson Disease. Journal of geriatric psychiatry and neurology 2015;28:210-217.

33. Levy R, Dubois B. Apathy and the Functional Anatomy of the Prefrontal Cortex-Basal Ganglia Circuits. Cerebral cortex 2006;16:916-928.

34. Chaudhuri KR, Schapira AH. Non-Motor Symptoms of Parkinson's Disease: Dopaminergic Pathophysiology and Treatment. The Lancet Neurology 2009;8:464-474.

35. Devos D, Moreau C, Maltête D, Lefaucheur R, Kreisler A, Eusebio A, et al. Rivastigmine in Apathetic but Dementia and Depression-Free Patients with Parkinson's Disease: A Double-Blind, Placebo-Controlled, Randomised Clinical Trial. Journal of Neurology, Neurosurgery \& Psychiatry 2014;85:668-674.

36. Aarsland D, Marsh L, Schrag A. Neuropsychiatric Symptoms in Parkinson's Disease. Movement Disorders 2009;24:2175-2186.

37. Rezak M. Current Pharmacotherapeutic Treatment Options in Parkinson's Disease. 
Disease-a-month 2007;53:214-222.

38. Maidment I, Fox C, Boustani M. Cholinesterase Inhibitors for Parkinson's Disease Dementia. The Cochrane Library 2006.

39. Dujardin K, Leentjens AF, Langlois C, Moonen AJ, Duits AA, Carette AS, et al. The Spectrum of Cognitive Disorders in Parkinson's Disease: A Data-Driven Approach. Movement Disorders 2013;28:183-189.

40. Ibarretxe-Bilbao N, Junque C, Tolosa E, Marti MJ, Valldeoriola F, Bargallo N, et al. Neuroanatomical Correlates of Impaired Decision-Making and Facial Emotion Recognition in Early Parkinson's Disease. Eur J Neurosci 2009;30:1162-1171.

41. Baggio HC, Segura B, Ibarretxe-Bilbao N, Valldeoriola F, Marti MJ, Compta Y, et al. Structural Correlates of Facial Emotion Recognition Deficits in Parkinson's Disease Patients. Neuropsychologia 2012;50:2121-2128.

42. Schroder C, Mobes J, Schutze M, Szymanowski F, Nager W, Bangert M, et al. Perception of Emotional Speech in Parkinson's Disease. Mov Disord 2006;21:1774-8.

43. Yoshimura N, Kawamura M, Masaoka Y, Homma I. The Amygdala of Patients with Parkinson's Disease Is Silent in Response to Fearful Facial Expressions. Neuroscience 2005;131:523-534.

44. Wieser MJ, Klupp E, Weyers P, Pauli P, Weise D, Zeller D, et al. Reduced Early Visual Emotion Discrimination as an Index of Diminished Emotion Processing in Parkinson's Disease? - Evidence from Event-Related Brain Potentials. Cortex 2012;48:1207-1217.

45. Garrido-Vásquez P, Pell MD, Paulmann S, Strecker K, Schwarz J, Kotz SA. An Erp Study of Vocal Emotion Processing in Asymmetric Parkinson's Disease. Social Cognitive and Affective Neuroscience 2013;8:918-927.

46. Lotze M, Reimold M, Heymans U, Laihinen A, Patt M, Halsband U. Reduced Ventrolateral Fmri Response During Observation of Emotional Gestures Related to the Degree of Dopaminergic Impairment in Parkinson Disease. J Cogn Neurosci 2009;21:1321-1331.

47. Delaveau P, Salgado-Pineda P, Witjas T, Micallef-Roll J, Fakra E, Azulay JP, et al. Dopaminergic Modulation of Amygdala Activity During Emotion Recognition in Patients with Parkinson Disease. J Clin Psychopharmacol 2009;29:548-554.

48. Dissanayaka NN, Sellbach A, Silburn PA, O'Sullivan JD, Marsh R, Mellick GD. Factors Associated with Depression in Parkinson's Disease. J Affect disorders 2011;132:82-88.

49. Riedel O, Heuser I, Klotsche J, Dodel R, Wittchen H-U. Occurrence Risk and Structure of Depression in Parkinson Disease with and without Dementia: Results from the Gepad Study. Journal of geriatric psychiatry and neurology 2010;23:27-34.

50. Becker C, Brobert G, Johansson S, Jick S, Meier C. Risk of Incident Depression in Patients with Parkinson Disease in the Uk. European Journal of Neurology 2011;18:448-453.

51. Gallagher DA, Schrag A. Psychosis, Apathy, Depression and Anxiety in Parkinson's Disease. Neurobiology of disease 2012;46:581-589.

52. Weintraub D, Moberg PJ, Duda JE, Katz IR, Stern MB. Recognition and Treatment of Depression in Parkinson's Disease. Journal of geriatric psychiatry and neurology 2003;16:178-183.

53. Richard I, McDermott M, Kurlan R, Lyness J, Como P, Pearson N, et al. A Randomized, Double-Blind, Placebo-Controlled Trial of Antidepressants in Parkinson Disease. Neurology 2012;78:1229-1236. 


\section{CHAPTER 2}

Neurobiological correlates of emotional Processing in Parkinson's DISEASE: A SYSTEMATIC REVIEW OF EXPERIMENTAL STUDIES

Anja J.H. Moonen, Anke Wijers, Kathy Dujardin, Albert F.G. Leentjens 


\begin{abstract}
Deficits in emotional processing in patients with Parkinson's disease (PD) have received increasing interest over the past decades. In this systematic review, we present the results of 16 behavioral studies that have examined the neurobiological base of emotional processing in PD. Multiple aspects of emotional processing have been studied, using a variety of research methods. Deficits in PD are mainly related to autonomic and perceptive processing of intense emotional stimuli, which is accompanied by structural and functional neurobiological abnormalities in predominantly ventral regions of affective neurocircuitry. These structures are more strongly dependent on dopaminergic neurotransmission than the dorsal structures of affective neurocircuitry, which are more related to the cognitive and regulatory aspects of emotion and appear to remain largely intact in PD patients. Considering the importance of active dopaminergic neurotransmission, PD can serve as a prolific model for studying the neurobiological correlates of normal human emotional behavior as well as psychiatric disorders such as anxiety, depression, and apathy. Moreover, the fact that PD patients are able to cognitively regulate or modulate their emotional responses despite reduced dopamine supplies, can have important implications for the treatment of affective disorders not only in PD patients but in the general population likewise.
\end{abstract}




\section{INTRODUCTION}

Non-motor manifestations in Parkinson's disease (PD) have been the focus of a growing number of scientific studies over the past decades. Apart from the characteristic motor symptoms such as tremor, hypokinesia, rigidity, and postural instability, PD patients frequently suffer from psychopathological syndromes, including affective disorders, cognitive deterioration, sleep disturbances and hallucinations. ${ }^{1}$ Moreover, several studies have shown that these non-motor symptoms affect the quality of life of PD patients to a greater extent than the motor symptoms and have a negative impact on the prognosis of the disease..$^{2-5}$

Even in the absence of clinical disturbances in mood and motivation, patients with PD encounter difficulties in generating and experiencing emotions. For instance, they often have difficulties in interpreting emotions in facial expressions, ${ }^{6-9}$ which may affect interaction with other people. Considering the disease-related neurological damage to the dopaminergic systems, which are thought to be critical in the processing of emotions, ${ }^{10}$ the possibility of a more extended emotional deficit in PD patients was raised. Experimental studies on this topic find rather mixed results. Several studies reported altered emotional functioning in PD patients, ${ }^{11-13}$ whereas other studies do not. ${ }^{14,15}$ Moreover, some studies report modality-specific deficits instead of a general dysfunction of emotional processing in PD patients ${ }^{11,13,16}$ which suggests the possible involvement of multiple neural substrates.

The present review aims to expand our understanding of the neurobiological base of emotional processing in PD by providing a systematic overview of experimental studies that have incorporated both behavioral and neurobiological correlates (e.g., brain activity, structural volume, sympathetic arousal).

\section{METHODS}

Search strategy

A systematic literature search was conducted in PubMed and PsycINFO, which was extended with searches of references listed in the reviewed papers. The entire timescale was used, which comprised all literature between 1965 and June 2015 (included). There were no language restrictions, so articles from any language were included. Animal studies were excluded. Our search resulted in a total of 3871 articles (without duplicates). For an overview of the Medical Subject Headings and free text words that were used in the search strategy, see Box 1. 
Box 1. Search strategy

\section{Search terms}
PubMed Parkinson*(title/abstract) OR Parkinson's disease (MeSH) AND (emotion* OR facial* OR arousal OR prosody OR subjective*) AND Humans (MeSH) NOT "review"(filter)

PsycINFO Parkinson*[title/abstract] AND (emotion* OR facial* OR arousal OR prosody OR subjective*)

\section{Selection of studies}

Papers were selected according to the following inclusion criteria: i) patients were diagnosed with idiopathic Parkinson's disease ii) emotional processing, measured in a behavioral task was the main outcome iii) the study included a neurobiological measure of emotional processing, iv) data analyses incorporated both the behavioral and neurobiological measurements of emotional processing. Papers dealing with the emotional effects of deep brain stimulation (DBS) or ablative brain surgery (e.g., pallidotomy, thalamotomy) were excluded from the present review. We only included papers from completed studies, hence papers reporting on interim analyses were excluded.

The abstracts from all 3871 articles were screened by two authors (AM and AW) based on the above-mentioned criteria. Full copies were screened from articles that could not be classified by abstract or title alone. All potential relevant articles were read in full and screened by two authors (AM and AW). In case of discrepancies between the two authors, consensus was reached after discussion, or by consulting a third author $(A L)$ who made the final decision.

For the selection process the authors followed the guidelines from the PRISMA statement (Preferred Reporting of Systematic reviews and Metaanalyses). ${ }^{17}$ Figure 1 illustrates the PRISMA Flow Diagram, which summarizes each step in the selection process (see Supplementary Table 1 for the PRISMA checklist). From the 3871 papers, 3825 could be excluded after reading the title and abstract alone. Further screening for eligibility resulted in 15 studies that were included for extensive review. One additional study was included after checking the reference lists of the included papers. 


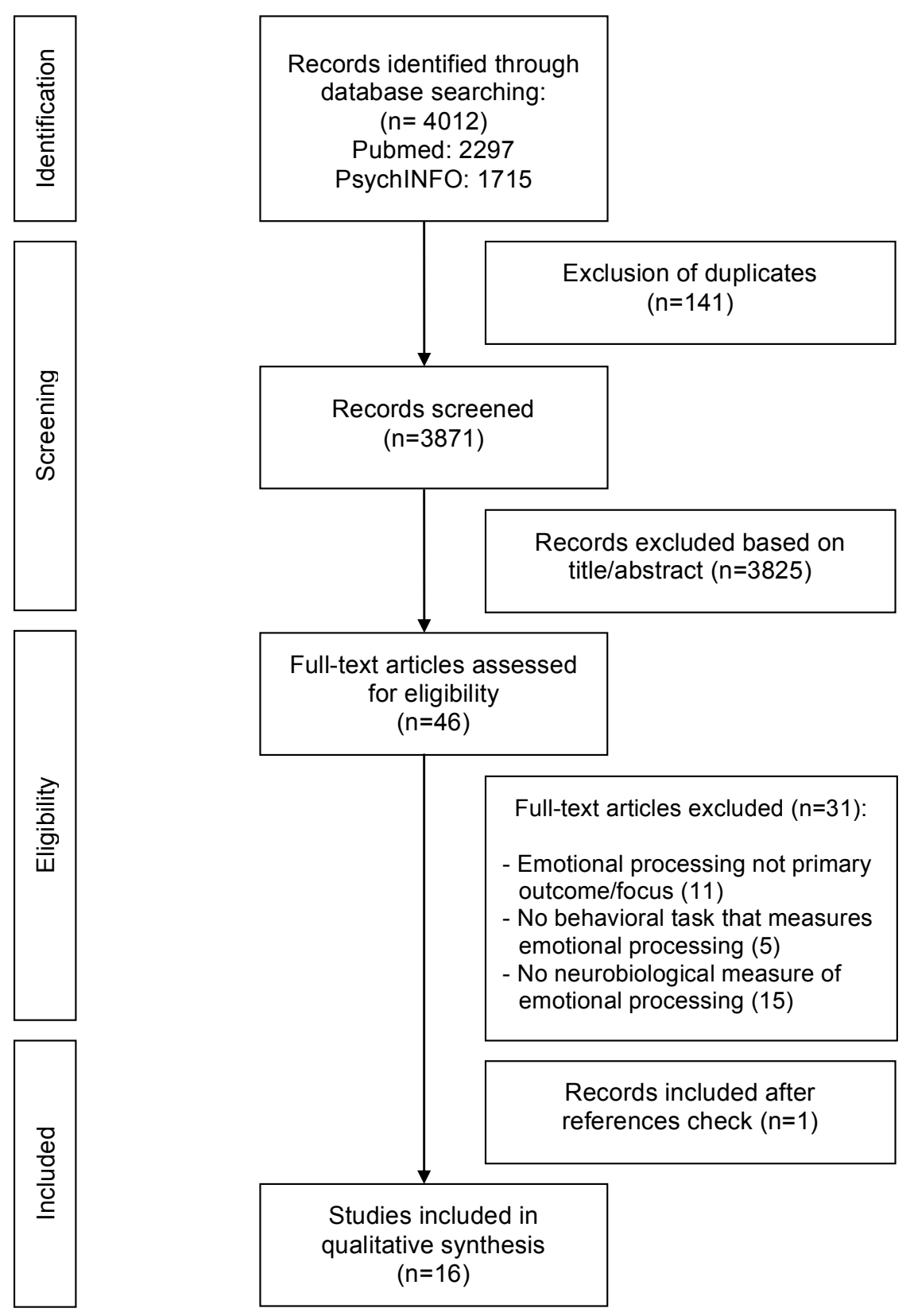

Figure 1. PRISMA Flow Diagram of search strategy

\section{Quality assessment}

At present, there is no 'gold standard' available for assessing the quality of nonrandomized quasi-experimental research. We therefore combined items from two checklists ${ }^{18,19}$ that have been systematically reviewed. ${ }^{20}$ We selected the items that specifically rated descriptive, statistical and internal validity, leaving out those items that were not relevant for our study (e.g., items on specific pharmaceutical issues). We then expanded our selection with four additional items from the STROBE 
statement, concerning study design, statistical interpretation of data, and power calculation. ${ }^{21}$ Items were scored as either good (2), moderate (1), or inadequate/undefined (0), which enabled us to compare the quality of, included studies mutually, despite the fact that we combined items from different checklists. The full checklist is included as supplementary material (Supplementary Table 2).

Two authors (AM and AW) reviewed the methodological quality of the included studies according to the selected items. In case of disagreement, consensus was reached after discussion, or by consulting a third author (AL) who made the final decision.

\section{RESULTS}

\section{Study characteristics}

The literature search resulted in 16 articles that were included for further review. All studies investigated emotional processing in patients with Parkinson's disease (PD) at a neurobiological level. Table 1 illustrates the characteristics and outcomes of the included studies. All studies were cross-sectional. They varied widely in experimental paradigms with multiple aspects of emotional processing being researched. A total of 10 studies investigated facial emotion recognition, 2 studies measured physiological arousal, 2 studies looked at emotional prosody recognition, and 2 studies used a multimodal audio-visual approach. Seven studies measured neurobiological correlates of emotional processing by using electro-encephalogram (EEG) and measuring the event-related potentials (ERP) or spectral modifications (1 study), 4 used blood-oxygen-level dependent functional magnetic resonance imaging (BOLDfMRI), 2 used structural magnetic resonance imaging (MRI), 2 used positron emission tomography (PET), and 1 used single photon emission CT (SPECT).

\section{Methodological quality}

Table 2 shows that the methodological quality varied among the included studies and did neither increase nor decrease with time. The mean quality score was 25.8 (range 11-32) out of 36 , with the majority of scores lying above 20 . Studies with a lower quality score poorly described their sample, design and outcomes and their subject groups were not adequately matched in terms of age, sex and education. Sample sizes ranged from 9 to 39 and none of the studies included a formal sample size calculation. Moreover, 8 studies did not report any indicators for strength of evidence and 12 studies lacked adequate adjustment for important confounding factors such 
as dopaminergic or psychiatric medication, disease severity, or presence of psychiatric disorders. However, the majority of the studies clearly specified their aim, sample characteristics, experimental design and outcomes, and provided extensive and clear interpretations of the reported findings. Given the limited number of included studies we decided not to exclude studies on the basis of an arbitrary cut-off on the quality score.

In the next section, the main results for each modality of emotional processing will be discussed. Per modality results are further subdivided by research method and are presented chronologically.

\section{Facial emotion recognition (FER)}

Functional imaging (fMRI)

Tessitore and colleagues conducted a blocked fMRI-experiment in which 9 early PD patients under dopamine replacement therapy (DRT) and 9 matched healthy controls had to match angry and fearful facial expressions. ${ }^{22}$ Despite equal task performances, the robust bilateral amygdala response found in healthy subjects was absent in PD patients who were temporarily depleted from DRT. After dopamine repletion, the amygdala response was partially restored, yet remained diminished compared to the healthy subjects, which indicates that reduced dopamine availability in the amygdala is associated with FER deficits.

In a more recent fMRI study, Lotze and colleagues reported that a sample of 9 early to advanced PD patients, assessed during their off state, made more errors than 9 age-matched healthy controls in an emotion recognition task (i.e., emotional vs. neutral gestures, faces included. ${ }^{23}$ In addition, PD patients showed decreased functional activity for observing emotional gestures in the left ventrolateral prefrontal cortex (VLPFC) and right superior temporal sulcus (STS). Additional PET scanning quantified less striatal dopamine transporter availability (DAT) in PD patients compared to healthy controls, with a specific association between reduced DAT in the left putamen and more severe motor impairment as well as more recognition errors in PD patients. Moreover, left putaminal DAT appeared to be positively correlated with left VLPFC activity, which indicates how disturbed dopaminergic neurotransmission can affect functional activity in this area during emotional gesture 


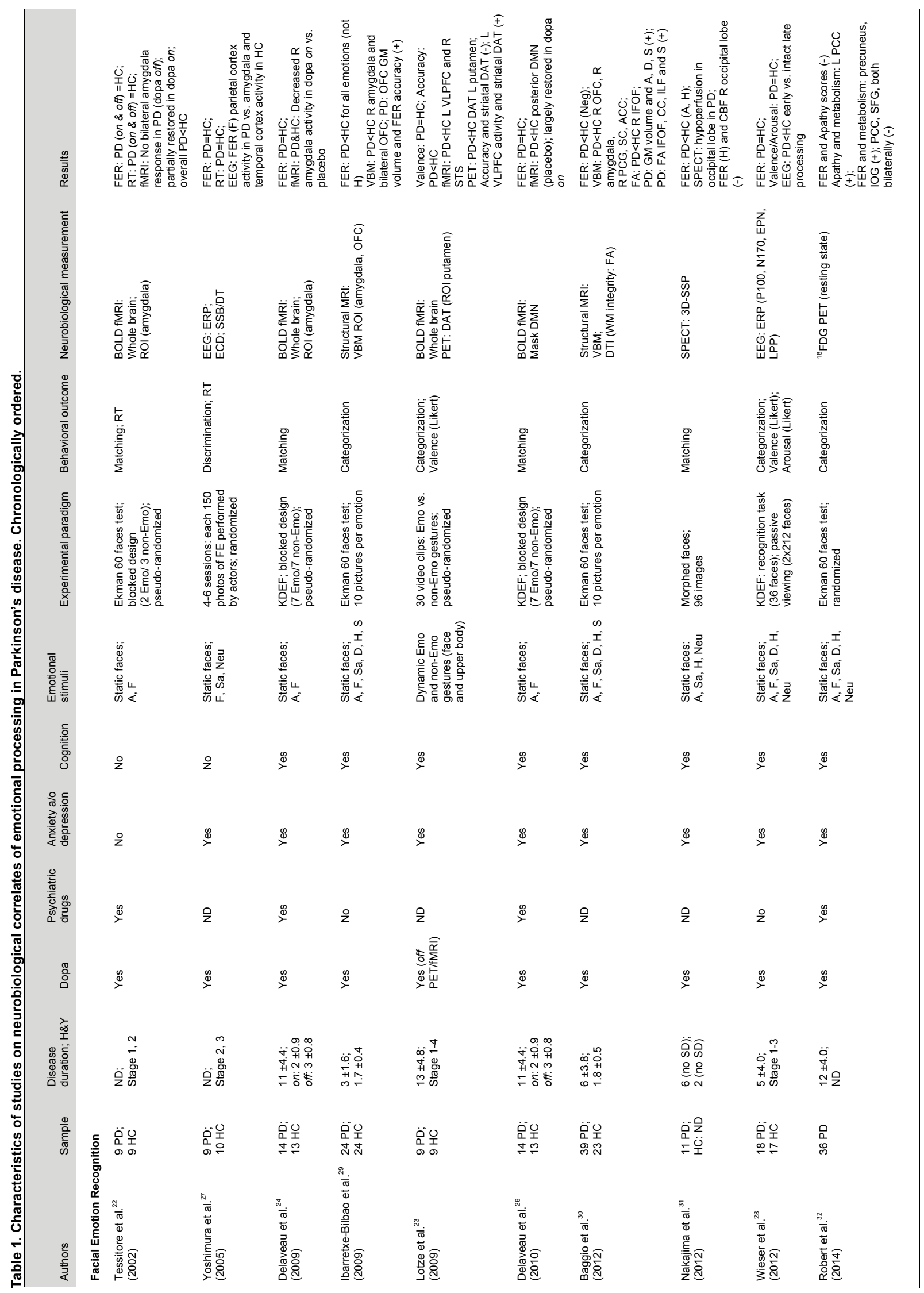




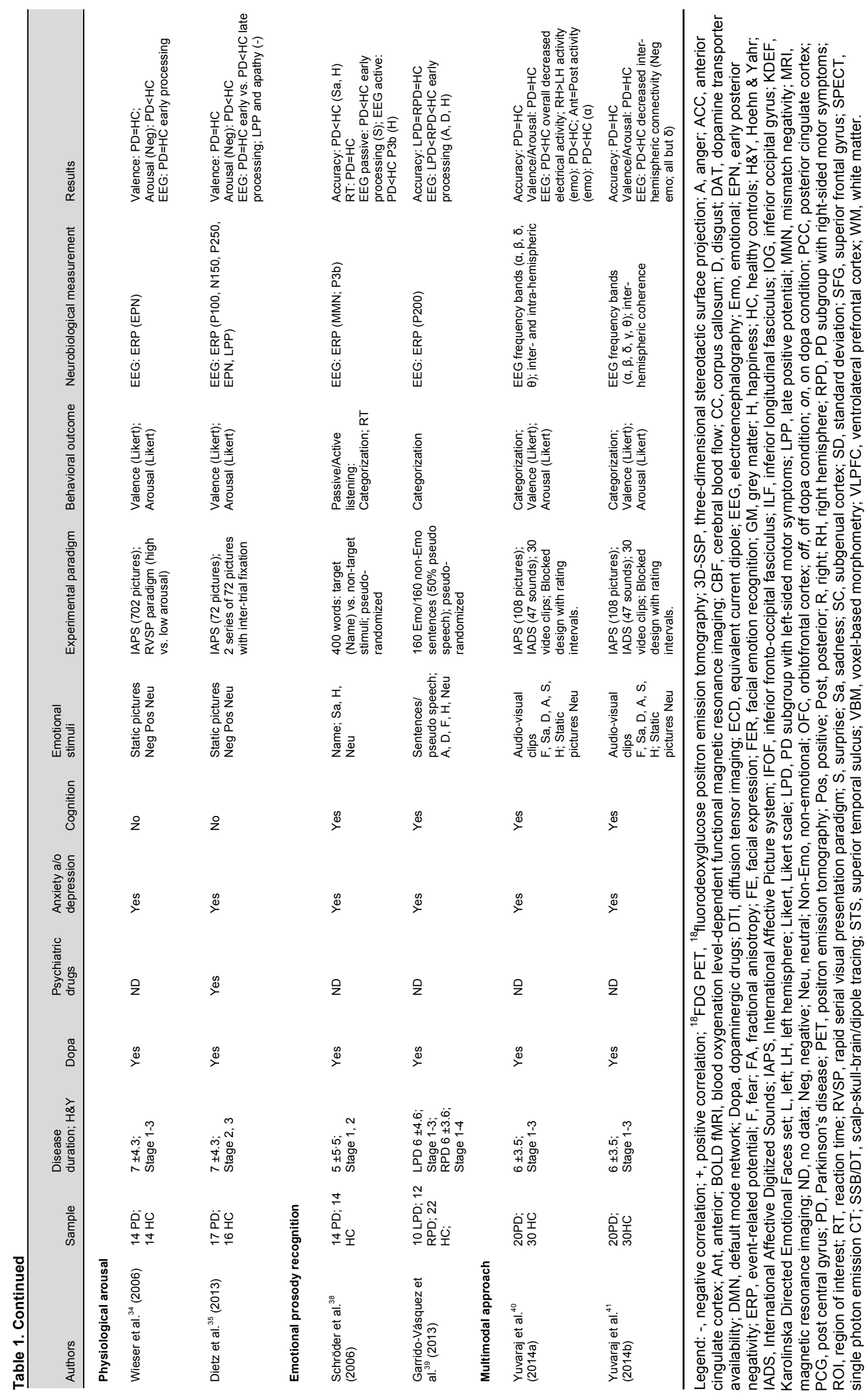


Table 2. Checklist methodological quality of included quasi-experimental studies

\begin{tabular}{|c|c|c|c|c|c|c|c|c|c|c|c|c|c|c|c|c|}
\hline \multirow[b]{2}{*}{ Items } & \multicolumn{16}{|c|}{ Author/Year } \\
\hline & 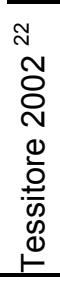 & 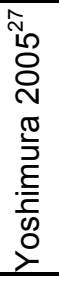 & 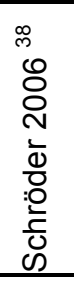 & 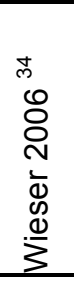 & 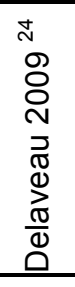 & 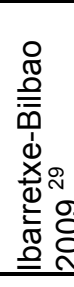 & 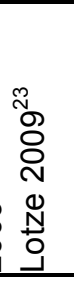 & 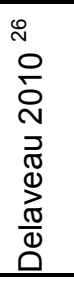 & 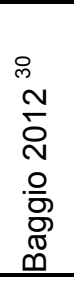 & 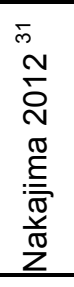 & 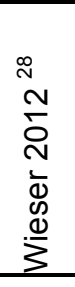 & 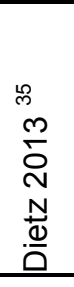 & 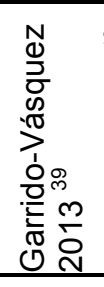 & 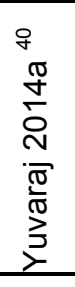 & 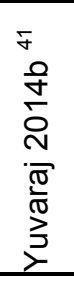 & 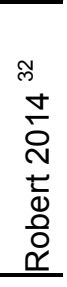 \\
\hline \multicolumn{17}{|l|}{ Reporting } \\
\hline $\begin{array}{l}\text { Hypothesis/aim/objective } \\
\text { clearly described }^{1}\end{array}$ & 2 & 2 & 2 & 2 & 2 & 2 & 2 & 2 & 2 & 1 & 2 & 2 & 2 & 2 & 1 & 2 \\
\hline $\begin{array}{l}\text { Main outcomes clearly } \\
\text { described Intro/Methods }{ }^{1}\end{array}$ & 2 & 2 & 2 & 2 & 2 & 2 & 1 & 2 & 2 & 1 & 2 & 2 & 2 & 2 & 1 & 2 \\
\hline $\begin{array}{l}\text { Subject characteristics } \\
\text { clearly described }\end{array}$ & 1 & 0 & 2 & 2 & 1 & 2 & 1 & 1 & 2 & 0 & 2 & 2 & 2 & 2 & 2 & 1 \\
\hline $\begin{array}{l}\text { Description study } \\
\text { design }^{3}\end{array}$ & 2 & 2 & 2 & 2 & 2 & 2 & 2 & 2 & 2 & 1 & 2 & 2 & 2 & 2 & 2 & 2 \\
\hline $\begin{array}{l}\text { Description/explanation } \\
\text { for drop-outs }\end{array}$ & 2 & 2 & 2 & 2 & 2 & 2 & 2 & 2 & 2 & 0 & 2 & 2 & 2 & 2 & 2 & 2 \\
\hline $\begin{array}{l}\text { Main findings clearly } \\
\text { described (no statistics) }\end{array}$ & 2 & 1 & 2 & 2 & 2 & 2 & 1 & 2 & 2 & 0 & 2 & 2 & 2 & 1 & 1 & 1 \\
\hline $\begin{array}{l}\text { Statistical methods } \\
\text { clearly described }^{2}\end{array}$ & 2 & 1 & 1 & 1 & 2 & 2 & 1 & 1 & 2 & 1 & 2 & 2 & 2 & 1 & 1 & 2 \\
\hline $\begin{array}{l}\text { Actual probability values } \\
\text { for main outcomes }{ }^{1}\end{array}$ & 0 & 0 & 1 & 1 & 2 & 2 & 1 & 1 & 2 & 2 & 2 & 1 & 1 & 2 & 2 & 1 \\
\hline \multicolumn{17}{|l|}{ Comparability } \\
\hline $\begin{array}{l}\text { Comparison/control } \\
\text { group included }^{2}\end{array}$ & 2 & 2 & 2 & 2 & 2 & 2 & 2 & 2 & 2 & 1 & 2 & 2 & 2 & 2 & 2 & 0 \\
\hline Adequate groupmatching ${ }^{2}$ & 2 & 0 & 2 & 2 & 0 & 2 & 1 & 0 & 1 & 0 & 1 & 0 & 2 & 1 & 1 & NA \\
\hline \multicolumn{17}{|c|}{$\begin{array}{l}\text { Presentation and } \\
\text { analyses of data }\end{array}$} \\
\hline $\begin{array}{l}\text { Appropriate statistical } \\
\text { tests }\end{array}$ & 2 & 2 & 2 & 2 & 2 & 2 & 2 & 2 & 2 & 1 & 2 & 2 & 2 & 2 & 2 & 2 \\
\hline $\begin{array}{l}\text { Adequate adjustment for } \\
\text { confounding }\end{array}$ & 1 & 0 & 0 & 0 & 1 & 2 & 0 & 1 & 2 & 0 & 1 & 2 & 0 & 1 & 0 & 2 \\
\hline $\begin{array}{l}\text { All comparisons have sam } \\
\text { number of subjects }{ }^{2}\end{array}$ & 1 & 2 & 2 & 1 & 1 & 1 & 1 & 1 & 0 & 0 & 1 & 1 & 1 & 1 & 1 & 1 \\
\hline $\begin{array}{l}\text { Descriptive measures } \\
\text { identified }^{2}\end{array}$ & 2 & 1 & 2 & 2 & 2 & 2 & 2 & 2 & 2 & 1 & 2 & 2 & 2 & 2 & 2 & 2 \\
\hline $\begin{array}{l}\text { Unplanned sub analyses } \\
\text { explained }\end{array}$ & 1 & 2 & 2 & 2 & 2 & 2 & 2 & 2 & 2 & 0 & 2 & 2 & 2 & 2 & 2 & 2 \\
\hline \multicolumn{17}{|c|}{ Interpretation } \\
\hline $\begin{array}{l}\text { Appropriate interpretation } \\
\text { of findings }{ }^{3}\end{array}$ & 2 & 1 & 1 & 2 & 2 & 2 & 1 & 2 & 2 & 1 & 2 & 2 & 2 & 2 & 1 & 2 \\
\hline $\begin{array}{l}\text { Assessment of strength } \\
\text { of evidence } \\
3\end{array}$ & 0 & 0 & 0 & 1 & 0 & 1 & 0 & 0 & 1 & 1 & 2 & 0 & 1 & 1 & 1 & 0 \\
\hline \multicolumn{17}{|l|}{ Power calculation } \\
\hline $\begin{array}{l}\text { Appropriate sample size } \\
\text { determination }{ }^{3}\end{array}$ & 0 & 0 & 0 & 0 & 0 & 0 & 0 & 0 & 0 & 0 & 0 & 0 & 0 & 0 & 0 & 0 \\
\hline Total quality score & 26 & 20 & 27 & 28 & 27 & 32 & 22 & 25 & 30 & 11 & 31 & 28 & 29 & 28 & 24 & 24 \\
\hline
\end{tabular}

Scoring: $0=$ inadequate/undefined, $1=$ moderate, $2=$ good, NA $=$ not applicable

${ }^{1}$ (Downs \& Black, 1998), ${ }^{2}$ (Reisch, et al., 1989), ${ }^{3}$ (Von Elm, et al., 2008) 
recognition. However, these patients were not re-assessed during their on state, hence the influence of levodopa treatment remains unknown here.

Delaveau and colleagues further investigated the role of dopaminergic modulation in FER in a sample of 14 non-depressed PD patients, treated with levodopa, and 13 non-matched healthy controls. ${ }^{24}$ Since non-depressed PD patients appear to have relatively spared mesolimbic dopaminergic projections, administration of levodopa as treatment of motor symptoms may result in overdosing limbic regions and consequently disrupt normal amygdala functioning, as shown previously in healthy subjects. ${ }^{25} \mathrm{PD}$ patients showed similar right amygdala activation as the controls during a FER task when administered under placebo. However, amygdala activation was indeed decreased in both groups after administration of levodopa.

In the same population, Delaveau and colleagues investigated the effect of levodopa on default mode network (DMN) deactivation during the same FER task. ${ }^{26}$ Whereas the control group showed the classical deactivation of cortical areas (e.g., medial prefrontal cortex and posterior cingulate cortex), PD patients under placebo did not. However, after levodopa administration, DMN deactivation improved significantly, which again indicates that dopamine levels can modulate brain activation in regions that are essential for perceptual emotional processing.

\section{Event-Related Potentials (ERP)}

Yoshimura and colleagues studied FER by measuring ERPs in a sample of 9 mild to moderate PD patients under DRT and 10 non-matched healthy controls. ${ }^{27}$ Although PD patients performed the task equally well as healthy controls, the ERP results showed that PD patients appeared to use different neural substrates for recognizing emotions in fearful facial expressions. More specifically, 7 out of 10 healthy subjects showed an increase in initial negative response (N1) of which the equivalent current dipoles were concentrated in the amygdala. In PD patients, on the other hand, N1 was centered bilaterally in the angular gyrus and supramarginal gyrus, and notably there was no neuronal activity in the amygdala. One could however question whether localization of generators with a dipole fit is suitable to identify activity in subcortical structures.

In a more recent ERP study by Wieser and colleagues, emotional recognition accuracy and affective ratings of facial expressions were again not impaired in a sample of 18 mild to moderate PD patients under DRT. ${ }^{28}$ The authors did, however, find indirect evidence for diminished early visual discrimination of emotional facial 
expressions in $\mathrm{PD}$, as reflected by the lack of increased early posterior activity in occipital regions that was present in 17 matched healthy controls. Yet, late cortical evaluative processing seemed to be intact, as PD patients showed no deficits in late components of the ERPs (i.e., late positive potentials) localized in parietal regions. The authors point to possible compensatory effects by appealing to other cortical regions, such as somatosensory and prefrontal areas, in order to successfully discriminate emotional facial expressions. They further hypothesize that the disturbed early perceptual processing of emotional facial expressions may be due to dopaminergic dysfunction in striato-thalamo-cortical circuits, which may evolve into a broader emotional deficit in more advanced stages of the disease.

\section{Structural imaging (MRI)}

Ibarretxe-Bilbao and colleagues found that 24 early PD patients under DRT scored significantly lower than 24 matched healthy controls in recognizing facial expressions for all basic emotions except happiness (i.e., fear, anger, sadness, surprise and disgust). ${ }^{29}$ In addition, voxel-based morphometry (VBM) region of interest analyses of grey matter (GM) volume revealed that PD patients showed significant GM volume loss in the right amygdala and bilateral OFC compared to healthy subjects. Interestingly, after correcting for total GM volume, a strong positive correlation between total FER performance in patients and OFC volume became apparent.

In a more recent structural imaging study by Baggio and colleagues, 39 early PD patients under DRT performed significantly worse than 23 age-matched healthy controls in recognizing negative facial expressions (i.e., fear, anger, disgust, sadness). ${ }^{30}$ VBM analyses further showed positive correlations between recognition accuracy and GM volume in the right OFC, amygdala, and dorsal postcentral gyrus for sadness; in the right fusiform gyrus, ventral striatum, and subgenual cortex for anger; and in the dorsal anterior cingulate cortex (ACC) for disgust identification. In addition, diffusion tensor imaging (DTI) data revealed a positive correlation between sadness identification and white matter density in the right frontal lobe, of which the latter was significantly reduced in PD patients.

\section{Single photon emission CT (SPECT)}

Nakajima and colleagues used SPECT in order to investigate brain perfusion in structures related to FER deficits in early to advanced PD. ${ }^{31}$ A sample of 11 PD patients under DRT performed worse than healthy subjects (sample size not 
reported) in recognizing angry and happy faces. The authors reported reduced cerebral blood flow in the occipital lobe in PD patients compared to healthy controls. However, results of this study were difficult to interpret due to incomplete description of subject characteristics, statistical methods, and their main findings (see Table 2).

\section{${ }^{18}$ Fluorodeoxyglucose positron emission topography $\left({ }^{18} \mathrm{FDG} P E T\right)$}

Robert and colleagues investigated the relation between severity of apathy and FER impairment in a sample of 36 early PD patients under DRT. ${ }^{32}$ Apathy scores appeared to be negatively correlated to overall FER performance, irrespective of emotional category. Furthermore, ${ }^{18} \mathrm{FDG}$ PET measurements showed that severity of apathy was positively correlated with increased activity in the left posterior cingulate gyrus (PCG), whereas FER impairment was correlated with low metabolic activity in the bilateral posterior cingulate cortex and the superior frontal gyrus. Conjunction analysis revealed the involvement of the right premotor cortex, right OFC, left middle frontal gyrus, and left PCG in both networks, supporting the association between apathy and FER impairment. However, as these results are based on correlation analyses only, they will need to be verified by group comparisons between PD patients with and without apathy. ${ }^{33}$

\section{Physiological arousal}

\section{Event-Related Potentials (ERP)}

Wieser and colleagues measured physiological arousal in response to a wide range of emotional and neutral pictures in 14 mild to moderate PD patients under DRT and 14 matched healthy controls. ${ }^{34}$ Patient subjective ratings revealed a blunted emotional response for highly arousing negative pictures. In contrast, ERP data showed that the early perceptual stages of emotional information processing did not differ between PD patients and healthy controls, as reflected by similar early posterior parietal-occipital negativity. The results indicate that PD patients can adequately receive input regarding visual emotional stimuli, but experience highly arousing stimuli at the behavioral level as less intense than healthy controls.

In a more recent ERP study, Dietz and colleagues found similar results in a sample of 17 mild to moderate PD patients under DRT. Patients again appeared to be less sensitive to highly arousing negative pictures (i.e., lower arousal ratings) compared to 16 significantly older healthy controls. ${ }^{35}$ Moreover, although emotionally 
arousing pictures usually elicit an increase in amplitude in late positive potentials (LPPs), ${ }^{36,37}$ in PD this modulation was only present in response to positive pictures (compared to neutral) and not for negative pictures. Interestingly, higher levels of apathy appeared to be associated with reduced centro-parietal LPP modulation in response to negative pictures. This finding implies that emotional dysfunction may only be apparent in the presence of a certain degree of apathy. Early occipital and frontal components of emotional processing were not impaired in PD patients, a finding that is in line with the results from Wieser and colleagues. ${ }^{34}$

\section{Emotional prosody recognition}

\section{Event-Related Potentials (ERP)}

Schröder and colleagues investigated the concept of emotional prosody (i.e., recognizing the emotional content of spoken language) in 14 mild PD patients under DRT and 14 matched healthy controls. ${ }^{38}$ They measured early and late ERP components of vocal emotion processing during an oddball experiment in which subjects had to categorize the emotional prosody of each target word (i.e., happy, sad, neutral). PD patients made more errors than healthy controls in recognizing sadly spoken words in a vocal emotional processing experiment. In addition, early ERP components showed disturbed preattentive processing of sad words, whereas late ERP components showed reduced reactivity in response to happy words. The authors point to striatal dopamine deficiency as a possible explanation for the impaired early preattentive processing of emotional prosody.

A more recent ERP study by Garrido-Vásquez and colleagues elaborated on this hypothesis by focusing on a possible functional lateralization of the striatum in processing affective vocal stimuli. ${ }^{39}$ The PD sample consisted of 10 PD patients with predominantly left-sided (LPD) and 12 PD patients with predominantly right-sighted (RPD) motor symptoms (all mild to moderate and under DRT), and 22 matched healthy controls. Contrary to Schröder and colleagues, ${ }^{38}$ PD patients performed equally well compared to healthy controls in categorizing different vocal emotions, although sad sounds were not included here. Data from early ERP components showed deviated ERP patterns (i.e., no P200 reduction) in LPD patients for almost all emotional categories. RPD patients and healthy controls showed no such deficit, which suggests that especially right striatal neurodegeneration might affect emotional prosody recognition. 


\section{Multimodal approach}

\section{Electro-encephalogram (EEG)}

Yuvaraj and colleagues performed an EEG power analysis in different frequency bands in 20 mild to moderate PD patients under DRT and 30 matched healthy controls who were presented audio-visual emotionally arousing stimuli. ${ }^{40,41}$ Subjective ratings of the valence and intensity of emotional stimuli did not differ between groups, nor was there a difference in recognition performance. EEG results revealed that both groups showed greater right than left hemisphere activity during emotional processing, yet patients showed an overall reduction of EEG power across all 6 basic emotions compared to healthy controls. ${ }^{41}$ In addition, PD patients showed reduced interhemispheric functional connectivity for negative emotions. ${ }^{40}$

\section{Discussion}

In this paper we systematically reviewed the evidence base of changes in emotional processing and its neurobiological correlates in patients with Parkinson's disease (PD). The included studies have investigated multiple aspects of emotional processing in PD such as facial emotion recognition, physiological arousal, and emotional prosody. In the majority of studies, PD patients showed no behavioral deficits in the ability to accurately recognize and respond to emotional stimuli. ${ }^{22,24,26-}$ 28,39-41 However, some studies did find impairments, ${ }^{23,29,30,34,38}$ which seemed to be highly dependent on sample characteristics and study designs, as well as on the specific aspect or phase of emotional processing that was measured.

Measurements of both structural and functional correlates of emotional processing have provided direct and indirect evidence for the involvement of a number of brain structures, including the orbitofrontal cortices, amygdala, anterior cingulate cortices, and the basal ganglia. Moreover, studies that used a dopaminergic intervention highlighted the importance of disrupted dopamine neurotransmission in emotional processing $\mathrm{PD} .^{22,24,26}$ In addition, ERP recordings showed that certain cortical processing stages were impaired in PD during emotional processing, whereas others were still intact ${ }^{28,34,35,38,39}$ or may even have switched to alternative brain regions. ${ }^{27}$ Which processing stages are affected was largely dependent on the experimental paradigm. Emotional processing deficits were not restricted to either positive or negative emotions, which again strongly depended on the type of stimuli or emotion investigated. It seems safe though to conclude that in 
general, emotional processing deficits in PD are related to highly arousing stimuli, rather than neutral or low arousing stimuli, independent of the valence of the emotion (positive or negative).

In the next section, the contribution of specific brain structures, mechanisms, and pathways in emotional processing will be described in more detail. In the final section, multiple neuropathological changes related to emotional dysfunction in PD will be incorporated into a theoretical framework on emotional processing in general.

\section{Amygdala}

Studies that investigated the involvement of the amygdala in emotional processing in PD found both structural and functional abnormalities. Amygdala volume appeared to be significantly reduced in PD patients ${ }^{29,30}$ and functional activity was disturbed in case of decreased dopaminergic input associated with degenerated parts of the nigrostriatal and mesolimbic dopaminergic pathways. ${ }^{22,24}$ Amygdala activity in response to emotional stimuli is known to be highly dependent on dopaminergic projections $^{10}$ and dopamine repletion can restore these deficiencies and subsequently improve perceptual emotion processing. ${ }^{22}$ In case of a novel or threatening stimulus, dopamine is released into the basolateral part of the amygdala, which in turn leads to suppression of prefrontal cortex inhibition. ${ }^{42}$ In PD, reduced dopamine projections to the prefrontal cortex prevent the amygdala from being disinhibited and subsequently may decrease its activity in response to intense emotional stimuli. ${ }^{43}$ In addition, amygdala projections to structures that mediate physiological levels of arousal, such as the locus coeruleus in the brainstem and the hypothalamus, may be reduced likewise, just as the eventual response to the emotional stimulus. ${ }^{34,35}$

\section{Orbitofrontal cortices}

Grey matter (GM) volume of the orbitofrontal cortex (OFC) appeared to be significantly reduced in PD patients, and was shown to be strongly correlated with worse facial emotion recognition. ${ }^{29,30}$ These findings are in line with results from both lesion and neuroimaging studies in healthy subjects showing that orbitofrontal cortices were heavily involved in the processing of intense emotional stimuli. ${ }^{44-48}$ Moreover, emotion recognition difficulties in PD patients may arise from reduced dopaminergic input from structures that have close interconnections with orbital prefrontal regions, such as the ventromedial caudate nucleus and substantia nigra 
pars reticulata $(\mathrm{SNpr}){ }^{49}$ As the disease progresses and the dopamine supply to orbital prefrontal regions further declines, PD patients may experience more severe abnormalities in emotional behavior, being particularly problematic for their social environment. ${ }^{50}$

\section{Anterior cingulate circuit}

Reduced ventral anterior cingulate cortex (VACC) and subgenual cortex GM volume was related to impaired recognition of negative emotions in $\mathrm{PD} .{ }^{30}$ Furthermore, reduced striatal dopamine transporter availability (DAT) in the ventral striatum was associated with decreased ventrolateral PFC (VLPFC) activity and impaired emotion recognition in $\mathrm{PD}$ patients. ${ }^{23}$ Together, these regions operate as an 'anterior cingulate circuit' in which the ventral regions in particular are heavily involved in the generation of emotional reactions, whereas the dorsal parts control active labeling of emotions and top-down cognitive regulation of the affective state. ${ }^{47,51-53}$ Functioning of this circuit is mainly dependent on dopaminergic input and output from the VACC and VLPFC to the ventral striatum. ${ }^{49,54}$ The ventral striatum, in turn, innervates the SNpr, ventral pallidum (VP), medial subthalamic nucleus (STN), and the internal section of the globus pallidus (GPi). ${ }^{49}$ Structures within this circuit have close connections to other parts of the limbic system, such as the amygdala, nucleus accumbens, hypothalamus, insula, hippocampus, and OFC. ${ }^{55}$ Neuropathological deficits within this circuit, such as reduced dopaminergic neurotransmission or decreased GM volume, both of which are seen in PD, evidently lead to remarkable impairments in emotional functioning.

\section{A neurobiological model for emotional processing deficits in $P D$}

The confrontation with an emotional stimulus encompasses different processes that together generate an appropriate behavioral response. According to Phillips and colleagues ${ }^{47}$ three main processes can be distinguished: 1) identification and appraisal of the emotional relevance of the stimulus; 2) production of a specific affective state and behavior (i.e., physiological arousal and emotional feelings); 3) top-down regulation of the affective state and emotional behavior (Figure 2). In addition, Ochsner and colleagues proposed an interaction between bottom-up emotional subcortical appraisal systems and top-down cortical cognitive control processes that eventually results in the production of an emotional response that is appropriate in terms of significance and context. ${ }^{52}$ 
Although a number of studies included in this review provide clear evidence for impaired autonomic emotional response generation, the more active identification and conscious labeling of emotions at the behavioral level is not necessarily disturbed likewise. The neurobiological models on emotional processing as described above may provide an explanation for this discrepancy, ${ }^{47,52,53}$ as they link the different aspects of emotional processing to the involvement of two separate neural systems: a ventral and dorsal system. Here, specific parts of a predominantly ventral system are critical for perceiving emotions and generation of an appropriate behavioral emotional response. These regions include the amygdala, ventral striatum, ventral ACC, OFC, and VLPFC, as well as regions that receive input from the aforementioned structures, such as the hypothalamus and locus coeruleus. On the other hand, the more dorsal regions, including the dorsolateral PFC, dorsomedial PFC, dorsal ACC, and hippocampus, are involved in top-down regulation of the emotional appraisal and affective state, which requires several cognitive processes. Hence, whenever early autonomic aspects of emotional processing are compromised, involvement of dorsal regions may serve as a compensatory top-down cognitive control mechanism in order to modulate our emotional responses. However, several studies included in this review highlighted the critical role of dopamine in perceptual emotional processing. In fact, significant evidence was found for dopaminergic modulation of brain activity, predominantly in the ventral structures of affective neurocircuitry. As such, the progressive degeneration of nigrostriatal, mesocortical and mesolimbic dopaminergic pathways in PD can affect emotional functionality of those structures remarkably, while the more dorsal regions remain relatively spared. Therefore, when incorporating findings from studies reviewed here into existing neurobiological models, we believe that dopamine operates as a critical component of emotional processing in PD (Figure 2).

Nevertheless, although there seems to be consensus on the involvement of a ventral and dorsal system in emotional processing, an open mind must be kept regarding the different theories on the exact disposition of the different neuronal structures within these systems. Morishita and colleagues, ${ }^{56}$ for instance, consider the amygdala and hippocampus as modulatory components in the circuit, rather than being part of the ventral or dorsal systems, respectively. ${ }^{47,53}$ Another unresolved issue concerns the role of the ventral PFC, the OFC in particular, in emotional processing. Apart from being involved in emotion recognition and the generation of an affective response, ${ }^{47}$ the OFC is also thought to be important for outcome-based 
regulation strategies, ${ }^{52,53}$ although the latter view seems to be more related to reward learning and decision-making rather than perceptual emotional processing. Finally, as some of the reviewed studies point to possible compensatory effects by appealing to other cortical regions, even outside the proposed network for emotional processing, specific pathways and functional interactions between brain structures as well as the involvement of different neurotransmitters needs more clarification.

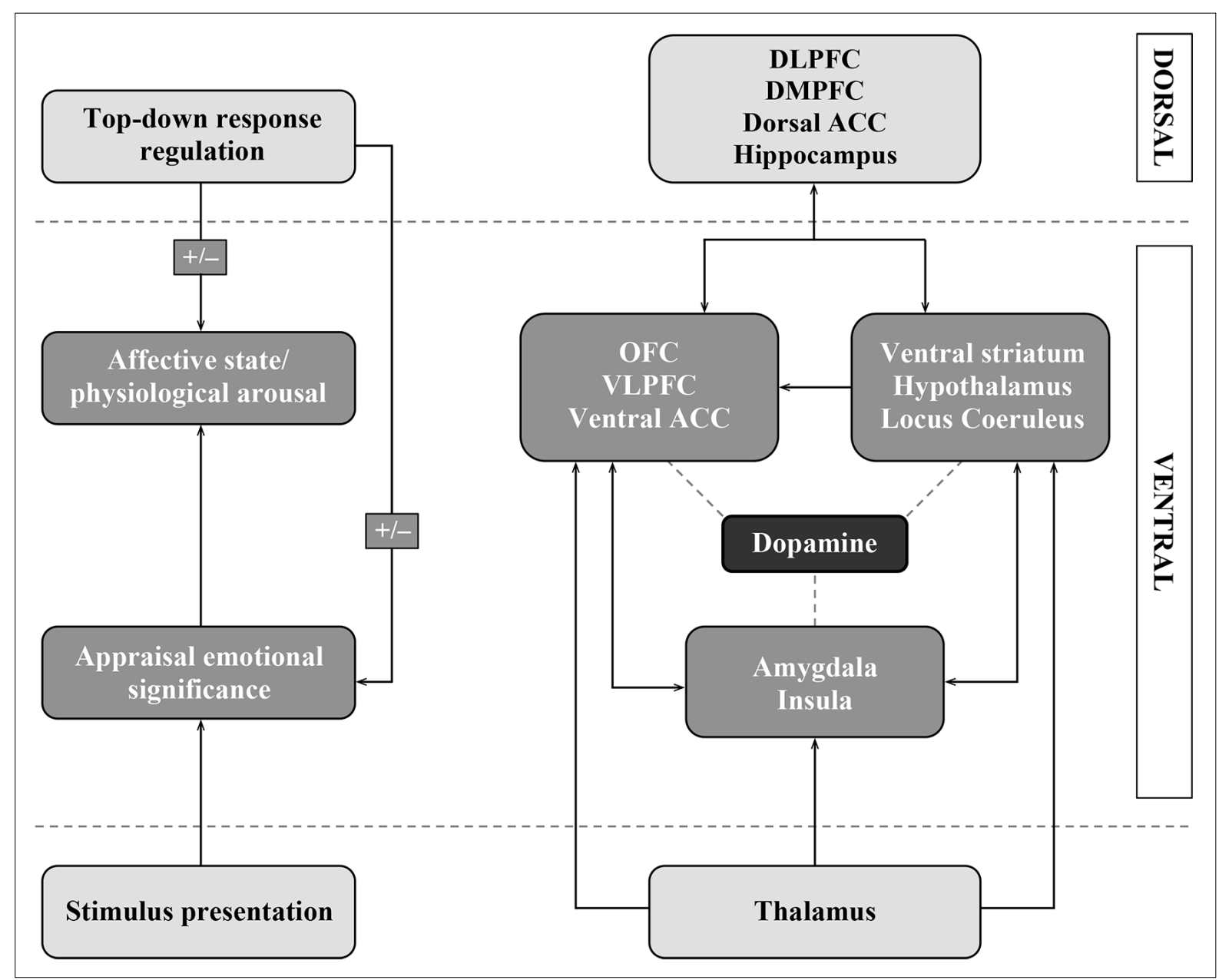

Figure 2. Schematic representation of emotional processing and its neurobiological base. We adjusted the schematic model that was initialized by Phillips and colleagues ${ }^{47}$ and included dopamine as the key element in two important emotional processes: accurate appraisal of the emotional significance of a stimulus and the production of an affective state and autonomic response. These processes are driven by predominantly ventral neural systems that strongly depend on sufficient dopamine supply. Top-down response regulation is controlled by a predominantly dorsal system. Both systems have reciprocal connections and therefore operate as a neuronal emotional circuit responsible for appropriate human emotional behavior.

Legend: DLPFC, dorsolateral prefrontal cortex; DMPFC, dorsomedial prefrontal cortex; ACC, anterior cingulate cortex; OFC, orbitofrontal cortex; VLPFC, ventrolateral prefrontal cortex. 


\section{Conclusions and future perspectives}

We reviewed studies on emotional processing in relation to its neurobiological correlates in Parkinson's disease (PD). These studies varied widely in methodology and clinical profiles, but presented clear evidence that several structural and functional correlates of emotional processing are disturbed in PD. The involvement of either ventral or dorsal neural systems of affective neurocircuitry may explain why certain aspects of emotional functioning are impaired in PD, whereas other aspects are not. Disturbance of dopamine neurotransmission, as we see in PD, can induce neurobiological abnormalities within this emotional circuit, particularly in the ventral regions.

Future studies on emotional processing in PD should consider several aspects. Optimal study designs require larger sample sizes, careful group matching, and accurate correction for confounding factors such as psychiatric medication, mood symptoms (i.e., depression, anxiety, apathy), and cognitive deterioration as they can have a substantial influence on a subjects' emotional response. Furthermore, considering the critical role of dopamine, dopaminergic replacement therapy is possibly the most determining factor for emotional processing in PD. Ideally, future studies should include both patient and control groups that are on and off dopaminergic medication in order to accurately define and control for the role of dopamine. Related to this, the stage and severity of the disease should be taken into account when interpreting study results. Medicated PD patients who are in more advanced stages of the disease, do not necessarily show more impairments in emotional processing than early PD patients who do not receive dopaminergic replacement therapy. ${ }^{8}$

We further suggest the use of standardized experimental paradigms on emotional processing, which makes it easier to correctly explain any inconsistencies across studies regarding behavioral results. Moreover, many neuroimaging designs seem to focus on specific brain areas such as the amygdala and OFC, which rules out the possibility to explore disturbances or even compensatory mechanisms elsewhere in the brain. Future EEG studies should consider the use of appropriate localization methods in order to draw informative conclusions on neural correlates of emotional processing. Together, these recommendations may increase reliability of findings and subsequently improve the quality of a study dramatically, especially since the latter here varied widely among studies. 
This review has some limitations. First our search was limited to Pubmed and PsycINFO, as we anticipated that due to the nature of the topic, studies would be published in journals with a Pubmed or PsycINFO listing. Also, we excluded studies in patients who received ablative surgery or deep brain stimulation. Although these studies may provide important information on affective deficits in PD, this may be more specific to changes associated with the intervention. Another limitation is that we only discussed the role of dopamine, whereas other neurotransmitter systems are likely to be involved as well. ${ }^{57}$ However, literature on the involvement of neurotransmitters other than dopamine in emotional processing in PD is very scarce. Finally, our quality assessment of the included quasi-experimental studies was based on a structured assessment that has not been validated. We do not expect that this led to a less critical appraisal of the studies since the list was more extensive than other available checklists.

In conclusion, this review reported important evidence that multiple aspects of emotional processing are disturbed in patients with PD. In general, PD patients show emotional processing deficits primarily for highly arousing stimuli. Deficits in PD are mainly related to autonomic and perceptive processing of intense emotional stimuli, which is accompanied by structural and functional neurobiological abnormalities in predominantly ventral regions of affective neurocircuitry. Regions within the dorsal system on the other hand that are more responsible for the cognitive and regulatory aspects of emotion, seem to remain largely intact in PD patients. Considering the fact that the ventral structures within this neuronal emotional circuit are strongly dependent on active dopaminergic neurotransmission, Parkinson's disease can serve as a prolific model for studying the neurobiological correlates of normal human emotional behavior. Moreover, the fact that PD patients are able to cognitively regulate or modulate their emotional responses despite reduced dopamine supplies, can have important implications for the treatment of affective disorders not only in this specific population but in the general population likewise. 


\section{REFERENCES}

1. Chaudhuri KR, Healy DG, Schapira AHV. Non-Motor Symptoms of Parkinson's Disease: Diagnosis and Management. The Lancet Neurology 2006;5:235-245.

2. Martinez-Martin P, Rodriguez-Blazquez C, Kurtis MM, Chaudhuri K. The Impact of Non-Motor Symptoms on Health-Related Quality of Life of Patients with Parkinson's Disease. Mov Disord 2011;26:399-406.

3. Reijnders JSAM, Ehrt U, Weber WEJ, Aarsland D, Leentjens AFG. A Systematic Review of Prevalence Studies of Depression in Parkinson's Disease. Mov Disord 2008;23:183-189.

4. Schrag A. Quality of Life and Depression in Parkinson's Disease. J Neurol Sci 2006;248:151-157.

5. Slawek J, Derejko M, Lass P. Factors Affecting the Quality of Life of Patients with Idiopathic Parkinson's Disease-a Cross-Sectional Study in an Outpatient Clinic Attendees. Parkinsonism Relat Disord 2005;11:465-468.

6. Ariatti A, Benuzzi F, Nichelli P. Recognition of Emotions from Visual and Prosodic Cues in Parkinson's Disease. Neurol Sci 2008;29:219-227.

7. Lawrence AD, Goerendt IK, Brooks DJ. Impaired Recognition of Facial Expressions of Anger in Parkinson's Disease Patients Acutely Withdrawn from Dopamine Replacement Therapy. Neuropsychologia 2007;45:65-74.

8. Sprengelmeyer R, Young AW, Mahn K, Schroeder U, Woitalla D, Buttner T, et al. Facial Expression Recognition in People with Medicated and Unmedicated Parkinson's Disease. Neuropsychologia 2003;41:1047-1057.

9. Yip JT, Lee TM, Ho SL, Tsang KL, Li LS. Emotion Recognition in Patients with Idiopathic Parkinson's Disease. Mov Disord 2003;18:1115-1122.

10. Hariri AR, Mattay VS, Tessitore A, Fera F, Weinberger DR. Neocortical Modulation of the Amygdala Response to Fearful Stimuli. Biological psychiatry 2003;53:494-501.

11. Clark US, Neargarder S, Cronin-Golomb A. Specific Impairments in the Recognition of Emotional Facial Expressions in Parkinson's Disease. Neuropsychologia 2008;46:2300-2309.

12. Dujardin K, Blairy S, Defebvre L, Duhem S, Noel Y, Hess U, et al. Deficits in Decoding Emotional Facial Expressions in Parkinson's Disease. Neuropsychologia 2004;42:239250.

13. Hillier A, Beversdorf DQ, Raymer AM, Williamson DJ, Heilman KM. Abnormal Emotional Word Ratings in Parkinson's Disease. Neurocase 2007;13:81-85.

14. Adolphs R, Schul R, Tranel D. Intact Recognition of Facial Emotion in Parkinson's Disease. Neuropsychology 1998;12:253-258.

15. Pell MD, Leonard CL. Facial Expression Decoding in Early Parkinson's Disease. Brain Res Cogn Brain Res 2005;23:327-340.

16. Kan Y, Kawamura M, Hasegawa Y, Mochizuki S, Nakamura K. Recognition of Emotion from Facial, Prosodic and Written Verbal Stimuli in Parkinson's Disease. Cortex 2002;38:623-630.

17. Moher D, Liberati A, Tetzlaff J, Altman DG. Preferred Reporting Items for Systematic Reviews and Meta-Analyses: The Prisma Statement. Annals of internal medicine 2009;151:264-269.

18. Reisch JS, Tyson JE, Mize SG. Aid to the Evaluation of Therapeutic Studies. Pediatrics 1989;84:815-827. 
19. Downs SH, Black N. The Feasibility of Creating a Checklist for the Assessment of the Methodological Quality Both of Randomised and Non-Randomised Studies of Health Care Interventions. Journal of epidemiology and community health 1998;52:377-384.

20. Deeks JJ, Dinnes J, D,Äôamico R, Sowden A, Sakarovitch C, Song F, et al. Evaluating Non-Randomised Intervention Studies. Health technology assessment 2003;7:1-179.

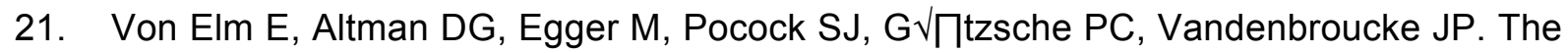
Strengthening the Reporting of Observational Studies in Epidemiology [Strobe] Statement: Guidelines for Reporting Observational Studies. Gaceta Sanitaria 2008;22:144-150.

22. Tessitore A, Hariri AR, Fera F, Smith WG, Chase TN, Hyde TM, et al. Dopamine Modulates the Response of the Human Amygdala: A Study in Parkinson's Disease. J Neurosci 2002;22:9099-9103.

23. Lotze M, Reimold M, Heymans U, Laihinen A, Patt M, Halsband U. Reduced Ventrolateral Fmri Response During Observation of Emotional Gestures Related to the Degree of Dopaminergic Impairment in Parkinson Disease. J Cogn Neurosci 2009;21:1321-1331.

24. Delaveau P, Salgado-Pineda P, Witjas T, Micallef-Roll J, Fakra E, Azulay JP, et al. Dopaminergic Modulation of Amygdala Activity During Emotion Recognition in Patients with Parkinson Disease. J Clin Psychopharmacol 2009;29:548-554.

25. Delaveau P, Salgado-Pineda P, Micallef-Roll J, Blin O. Amygdala Activation Modulated by Levodopa During Emotional Recognition Processing in Healthy Volunteers: A Double-Blind, Placebo-Controlled Study. J Clin Psychopharmacol 2007;27:692-697.

26. Delaveau P, Salgado-Pineda P, Fossati P, Witjas T, Azulay JP, Blin O. Dopaminergic Modulation of the Default Mode Network in Parkinson's Disease. Eur Neuropsychopharmacol 2010;20:784-792.

27. Yoshimura N, Kawamura M, Masaoka Y, Homma I. The Amygdala of Patients with Parkinson's Disease Is Silent in Response to Fearful Facial Expressions. Neuroscience 2005;131:523-534.

28. Wieser MJ, Klupp E, Weyers P, Pauli P, Weise D, Zeller D, et al. Reduced Early Visual Emotion Discrimination as an Index of Diminished Emotion Processing in Parkinson's Disease? - Evidence from Event-Related Brain Potentials. Cortex 2012;48:1207-1217.

29. Ibarretxe-Bilbao N, Junque C, Tolosa E, Marti MJ, Valldeoriola F, Bargallo N, et al. Neuroanatomical Correlates of Impaired Decision-Making and Facial Emotion Recognition in Early Parkinson's Disease. Eur J Neurosci 2009;30:1162-1171.

30. Baggio HC, Segura B, Ibarretxe-Bilbao N, Valldeoriola F, Marti MJ, Compta Y, et al. Structural Correlates of Facial Emotion Recognition Deficits in Parkinson's Disease Patients. Neuropsychologia 2012;50:2121-2128.

31. Nakajima H, Tsujino, A., Doi, H., Tateishi, Y., Motomura, M., Shinohara, K., Satoh, A., Tsujihata, M., Kawakami, A. An Impairment of Recognizing Emotional Facial Expressions in Parkinson's Disease. Nagasaki University School of Medicine 2012; Volume 57.

32. Robert G, Le Jeune F, Dondaine T, Drapier S, Péron J, Lozachmeur C, et al. Apathy and Impaired Emotional Facial Recognition Networks Overlap in Parkinson's Disease: A Pet Study with Conjunction Analyses. J Neurol, Neurosurg Ps 2014;85:1153-1158.

33. Dujardin K, Lopes R. Apathy and Impaired Recognition of Emotion: Are They Related in Parkinson's Disease? J Neurol, Neurosurg Ps 2014;85:1061. 
34. Wieser MJ, Muhlberger A, Alpers GW, Macht M, Ellgring H, Pauli P. Emotion Processing in Parkinson's Disease: Dissociation between Early Neuronal Processing and Explicit Ratings. Clin Neurophysiol 2006;117:94-102.

35. Dietz J, Bradley MM, Jones J, Okun MS, Perlstein WM, Bowers D. The Late Positive Potential, Emotion and Apathy in Parkinson's Disease. Neuropsychologia 2013;51:960966.

36. Bradley MM. Natural Selective Attention: Orienting and Emotion. Psychophysiology 2009;46:1-11.

37. Cuthbert BN, Schupp HT, Bradley MM, Birbaumer N, Lang PJ. Brain Potentials in Affective Picture Processing: Covariation with Autonomic Arousal and Affective Report. Biological psychology 2000;52:95-111.

38. Schröder C, Mobes J, Schutze M, Szymanowski F, Nager W, Bangert M, et al. Perception of Emotional Speech in Parkinson's Disease. Mov Disord 2006;21:1774-8.

39. Garrido-Vásquez P, Pell MD, Paulmann S, Strecker K, Schwarz J, Kotz SA. An Erp Study of Vocal Emotion Processing in Asymmetric Parkinson's Disease. Social Cognitive and Affective Neuroscience 2013;8:918-927.

40. Yuvaraj R, Murugappan M, Ibrahim NM, Sundaraj K, Omar MI, Mohamad K, et al. Inter-Hemispheric Eeg Coherence Analysis in Parkinson's Disease: Assessing Brain Activity During Emotion Processing. Journal of Neural Transmission 2014a;1-16.

41. Yuvaraj R, Murugappan M, Ibrahim NM, Iqbal M, Sundaraj K, Mohamad K, et al. On the Analysis of Eeg Power, Frequency and Asymmetry in Parkinson's Disease During Emotion Processing. Behav. brain Funct 2014b;10:12.

42. Marowsky A, Yanagawa Y, Obata K, Vogt KE. A Specialized Subclass of Interneurons Mediates Dopaminergic Facilitation of Amygdala Function. Neuron 2005;48:1025-1037.

43. Bowers D, Miller K, Mikos A, Kirsch-Darrow L, Springer U, Fernandez H, et al. Startling Facts About Emotion in Parkinson's Disease: Blunted Reactivity to Aversive Stimuli. Brain 2006;129:3356-3365.

44. Adolphs R. Neural Systems for Recognizing Emotion. Curr Opin Neurobiol 2002a;12:169-177.

45. Aldhafeeri FM, Mackenzie I, Kay T, Alghamdi J, Sluming V. Regional Brain Responses to Pleasant and Unpleasant laps Pictures: Different Networks. Neuroscience letters 2012;512:94-98.

46. Öngür D, Price J. The Organization of Networks within the Orbital and Medial Prefrontal Cortex of Rats, Monkeys and Humans. Cerebral cortex 2000;10:206-219.

47. Phillips ML, Drevets WC, Rauch SL, Lane R. Neurobiology of Emotion Perception I: The Neural Basis of Normal Emotion Perception. Biol Psychiat 2003;54:504-514.

48. Vuilleumier P, Armony JL, Driver J, Dolan RJ. Effects of Attention and Emotion on Face Processing in the Human Brain: An Event-Related Fmri Study. Neuron 2001;30:829-841.

49. Alexander GE, DeLong MR, Strick PL. Parallel Organization of Functionally Segregated Circuits Linking Basal Ganglia and Cortex. Annual review of neuroscience 1986;9:357-381.

50. Adolphs R. Recognizing Emotion from Facial Expressions: Psychological and Neurological Mechanisms. Behav Cogn Neurosci R 2002b;1:21-62.

51. Bush G, Luu P, Posner MI. Cognitive and Emotional Influences in Anterior Cingulate Cortex. Trends in cognitive sciences 2000;4:215-222. 
52. Ochsner KN, Gross JJ. The Neural Architecture of Emotion Regulation. Handbook of emotion regulation 2007;1:87-109.

53. Phillips ML, Ladouceur CD, Drevets WC. A Neural Model of Voluntary and Automatic Emotion Regulation: Implications for Understanding the Pathophysiology and Neurodevelopment of Bipolar Disorder. Molecular psychiatry 2008;13:833-857.

54. Bonelli RM, Cummings JL. Frontal-Subcortical Circuitry and Behavior. Dialogues in clinical neuroscience 2007;9:141.

55. Devinsky O, Morrell MJ, Vogt BA. Review Article Contributions of Anterior Cingulate Cortex to Behaviour. Brain 1995;118:279-306.

56. Morishita T, Fayad S, Higuchi M-a, Nestor K, Foote K. Deep Brain Stimulation for Treatment-Resistant Depression: Systematic Review of Clinical Outcomes. Neurotherapeutics 2014;11:475-484.

57. Ring H, Serra-Mestres J. Neuropsychiatry of the Basal Ganglia. Journal of Neurology, Neurosurgery \& Psychiatry 2002;72:12-21. 



\section{ChAPTER 3}

AN FMRI STUDY INTO THE NEUROBIOLOGY OF EMOTIONAL PROCESSING IN

PARKINSON'S DISEASE: DOES INCREASED MEDIAL PREFRONTAL CORTEX ACTIVATION COMPENSATE FOR STRIATAL DYSFUNCTION?

Anja J.H. Moonen, Peter H. Weiss, Michael Wiesing, Gereon R. Fink, Jennifer S.A.M. Reijnders, Wim M. Weber, Ralph Weidner, Albert F.G. Leentjens 


\section{ABSTRACT}

Introduction: Parkinson's disease (PD) is a complex neurodegenerative disorder. Apart from a progressive decline of motor functions, PD is also characterized by nonmotor symptoms, including disturbed processing of emotions. To date, however, the wealth of studies exploring the pathophysiological mechanisms underlying motor dysfunction is contrasted by the dearth of studies investigating emotional dysfunction in PD. This study aims at assessing emotional processing and its neurobiological correlates in patients suffering from PD by using blood-oxygen level-dependent functional magnetic resonance imaging (BOLD-fMRI) with the focus on how medicated PD patients may achieve normal emotional responsiveness despite basal ganglia dysfunction.

Methods: Nineteen medicated patients with mild to moderate PD (without dementia or depression) and 19 matched healthy controls (HCs) passively viewed positive, negative, and neutral pictures in an event-related BOLD-fMRI study. Individual subjective ratings of valence and arousal levels for these pictures were obtained right after the scanning.

Results: PD showed no deficits in emotional processing at the behavioral level, as their valence and arousal ratings were similar to HCs. Yet, PD patients showed decreased activation of the bilateral posterior putamen and increased activation in the right dorsomedial prefrontal cortex (dmPFC), compared to control subjects, most pronounced for highly arousing emotional stimuli. Regression analyses revealed that these differences in functional activity were independent of symptoms of apathy, depression, and anxiety, and there was no significant influence of the use of levodopa, disease duration, or severity of motor symptoms on the functional reactivity in patients.

Conclusion: While the reduced striatal activity is likely to be intimately related to the pathophysiology of PD, the increase in medial PFC activity may well underlie a compensatory top-down mechanism utilising cognitive control to restore emotional processing at the behavioural level, despite striatal dysfunction. These results may impact upon current treatment strategies of affective disorders in PD. Data suggest that therapeutic interventions that rely on cognitive control mechanisms may be particularly useful for PD who are cognitively intact, as they may benefit from a compensatory influence of prefrontal areas. Future neuroimaging studies into the neurobiology of emotional processing in PD should further explore functional connectivity patterns between frontal and subcortical limbic structures. 


\section{INTRODUCTION}

In addition to the characteristic motor symptoms such as tremor, hypokinesia, rigidity, and postural instability, patients suffering from Parkinson's disease (PD) frequently encounter neuropsychiatric syndromes, such as affective disorders, cognitive deterioration, sleep disturbances, and hallucinations. ${ }^{1}$ Several studies have shown that non-motor symptoms in PD can have an even larger impact on the patients' quality of life and her/his prognosis than the motor symptoms. ${ }^{2-5}$

Even in the absence of a psychopathological diagnosis, PD patients may exhibit disturbed emotional processing. Emotional processing refers to the conscious and unconscious processes of recognizing, experiencing, and expressing emotions. ${ }^{6}$ Recent studies, which examined whether emotional processing is impaired in PD patients, provided conflicting data. The majority of studies report an intact ability at the behavioral level of PD patients to explicitly recognize and categorize emotions, but an impaired ability to generate autonomic emotional responses. ${ }^{7-14}$ However, other studies reported behavioral impairments, often especially for negative emotions. With respect to the neural underpinnings of disturbed emotion processing in $\mathrm{PD}$, some of the studies failed to observe structural or functional deficits, ${ }^{15}$ while others reported reduced grey matter volume, ${ }^{16,17}$ or region-specific alterations of activity on electroencephalogram (EEG) or functional magnetic resonance imaging $(\mathrm{fMRI}) .^{7-11,18-24}$ Especially regions within frontal-subcortical limbic circuitry including the amygdala, the orbitofrontal cortex (OFC), the ventral anterior cingulate cortex ( $\mathrm{AACC}$ ), the ventral striatum, and the ventrolateral prefrontal cortex (VIPFC) have been described to be affected in PD. All these structures are part of the "emotional circuitry" and depend upon dopaminergic projections and are therefore specifically affected by reduced dopaminergic neurotransmission in PD.

Findings of a largely intact ability in PD patients to explicitly recognize and categorize emotions (despite the above mentioned neurobiological alterations in emotion processing related brain regions) raise the question whether compensational neural mechanisms or behavioral strategies are effective in PD. The present study aimed to address this question by investigating (implicit) emotional processing and its neurobiological correlates in 19 medicated PD patients and 19 matched healthy controls using event-related blood-oxygen-level dependent fMRI (BOLD-fMRI). We hypothesized that (compared to healthy controls) PD patients would show intact explicit emotional processing at the behavioural level. Furthermore, by comparing the neural activation patterns of PD patients and controls using BOLD-fMRI, we expected 
to find evidence for deficient neural processing in striatal and limbic brain areas involved in emotional processing, such as the amygdala and other structures of the affective neurocircuitry. ${ }^{6}$ We further hypothesized that compensatory neural mechanisms during implicit emotional processing in PD occur, thereby enabling intact emotion recognition abilities at the behavioural level.

\section{Methods}

\section{Participants}

Twenty-one patients with idiopathic PD and 21 matched healthy controls participated in the study. Patients were recruited from the Movement Disorders Clinic of the Maastricht University Medical Centre (MUMC). Controls were recruited from a preexisting database of healthy volunteers generated at the Department of Psychiatry of the MUMC to match the patient group with respect to age, gender, and education. All assessments and scanning sessions took place at the Institute for Neuroscience and Medicine, Research Centre Jülich, Germany.

To be eligible for participation, patients had to fulfill the Queen Square Brain Bank diagnostic criteria for PD. ${ }^{25}$ Patients had to be on stable doses of antiparkinsonian medication for at least one month. Patients with neurodegenerative disorders other than PD or controls with neurodegenerative disorders were excluded. For all subjects, the following additional exclusion criteria were applied: major depressive disorder, as defined by the criteria of the fourth edition of the Diagnostic and Statistical Manual (DSM-IV) of the American Psychiatric Association (APA), ${ }^{26}$ cognitive deterioration operationalized as a score of $<26$ on the Mini Mental State Examination (MMSE), ${ }^{27}$ contra-indications for MRI, and the presence of alcoholand/or drugs abuse. Written informed consent was obtained prior to participation and according to the guidelines of the Declaration of Helsinki. This study was approved by the ethics committee of the Ärztekammer Nordrhein (2012188).

Careful inspection of the imaging data resulted in the exclusion of two PD patients; one due to excessive head motion resulting in movement-related artifacts, and one because of a structural brain abnormality (i.e., a lacunar infarct in the right striatum). Subsequently, the two matching controls were excluded likewise. The remaining study sample thus consisted of 19 PD patients (13 male; mean age $=60.2$ \pm 9.6 years) and 19 matched healthy controls (13 male, mean age $=60.8 \pm 9.9$ years). All patients were assessed in their 'on' state and were taking stable doses of antiparkinsonian medication, consisting of different combinations of L-dopa $(n=17)$, L- 
dopa with COMT inhibitor $(n=1)$, dopamine agonists $(n=11)$, MAO inhibitors $(n=5)$, amantadine $(n=3)$, and anticholinergic drugs $(n=2)$. Levodopa equivalent daily doses (LEDD) were calculated in order to control for possible medication effects. ${ }^{28}$ None of the controls were taking antidepressants, compared to only one patient, yet in the absence of a major depressive disorder or another current psychiatric disorder.

Table 1 summarizes the demographic and clinical characteristics of the subjects. PD patients and controls did not differ in terms of age, gender, and education, which indicates that groups were well matched. In addition, both groups showed comparable scores for global cognition (MMSE) and executive functioning (Frontal Assessment Battery, $\mathrm{FAB}^{29}$ ). Compared to controls, PD patients showed significantly higher scores, yet still on a subclinical level, for depression (Hamilton Depression Rating Scale, HAMD ${ }^{30}$ ), anxiety (Hamilton Anxiety Rating Scale, HARS ${ }^{31}$; Parkinson Anxiety Scale total score, PAS ${ }^{32}$ ), particularly more episodic anxiety (PAS episodic), and apathy (Lille Apathy Rating Scale, LARS ${ }^{33}$ ). However, based on the proposed criteria for Apathy ${ }^{34}$, five patients (26.3\%) could be diagnosed with clinical apathy, compared to zero in the control group.

Table 1 further shows that the average disease duration in PD patients (postdiagnosis) was $5.3 \pm 3.9$ years (range $1-15$ years), with patients exhibiting mild to moderate severity of motor symptoms (Hoehn\&Yahr ${ }^{35}$ stages 1-3, median 2.5) and an average motor score of $23.8 \pm 8.6$ (Unified Parkinson Disease Rating Scale section 3, UPDRS-III ${ }^{36}$ ).

\section{Experimental design}

\section{Stimulus material}

In this cross-sectional study, the stimuli consisted of 195 color pictures that were selected from the International Affective Picture System (IAPS) ${ }^{37}$ based on their normative valence and arousal ratings, the two dimensions that define emotional stimuli. 'Valence' refers to the nature of emotional activation: positive, neutral or negative, whereas 'arousal' refers to the intensity of emotional activation, ranging from low to high arousal. ${ }^{38}$ The paradigm consisted of three emotional conditions: 65 highly arousing negative, 65 highly arousing positive, and 65 low arousing neutral pictures. The emotional conditions were balanced with respect to stimulus complexity 
Table 1. Demographic and clinical characteristics of Parkinson patients and matched healthy controls

\begin{tabular}{|c|c|c|c|}
\hline Characteristics & Parkinson $(n=19)$ & Controls $(n=19)$ & $P$-value \\
\hline Age & $60.2(9.6)$ & $60.8(9.9)$ & 0.86 \\
\hline Gender (\% male) & $68.4 \%$ & $68.4 \%$ & 1.00 \\
\hline Education (years) & $13.2(2.7)$ & $13.95(4.0)$ & 0.48 \\
\hline Handedness (right) & 17 & 17 & 1.00 \\
\hline HAMD & $5.2(4.5)$ & $2.5(2.6)$ & $0.03^{*}$ \\
\hline HARS & $5.7(4.3)$ & $2.2(2.2)$ & $0.01^{*}$ \\
\hline Total score & $8.3(6.7)$ & $4.3(4.7)$ & $0.04^{*}$ \\
\hline Persistent & $5.4(4.4)$ & $3.3(3.5)$ & 0.11 \\
\hline Episodic & $1.5(1.8)$ & $0.5(1.1)$ & $0.04^{*}$ \\
\hline Avoidance & $1.4(2.1)$ & $0.5(1.0)$ & 0.19 \\
\hline MMSE & $29.1(1.2)$ & $29.4(0.8)$ & 0.35 \\
\hline FAB & $16.8(1.3)$ & $16.5(1.3)$ & 0.55 \\
\hline LARS & $-23.6(7.7)$ & $-28.7(5.1)$ & $0.04^{*}$ \\
\hline Apathy Criteria (\% yes) & $26.3 \%$ & $0.0 \%$ & $0.02^{*}$ \\
\hline UPDRS II & $12.0(5.3)$ & -- & -- \\
\hline UPDRS III & $23.8(8.6)$ & -- & -- \\
\hline UPDRS IV & $2.3(2.2)$ & -- & -- \\
\hline Schwab \& England & $84.7(7.4)$ & -- & -- \\
\hline Hoehn-Yahr (median) & $2.5(0.5 ;$ range $1-3)$ & -- & -- \\
\hline LEDD (mg/day) & $448.4(213.6)$ & -- & -- \\
\hline
\end{tabular}

Note: Means and standard deviations. HAMD, Hamilton Rating Scale for Depression; HARS, Hamilton Anxiety Rating Scale; PAS, Parkinson Anxiety Scale; MMSE, Mini Mental State Examination; FAB, Frontal Assessment Battery; LARS, Lille Apathy Rating Scale; UPDRS, Unified Parkinson's Disease Rating Scale (section II-IV); LEDD, levodopa equivalent daily dose. * Significant group difference at $P<0.05$.

colors, presence of human beings and animals. Selected negative pictures included mutilations, threatening animals, human violence etc., with a mean valence rating of 2.3 (SD 0.6) and a mean arousal rating of 6.4 (SD 0.5). Positive pictures included babies, couples, food, sports activities etc., with a mean valence rating of 7.3 (SD 0.5 ) and a mean arousal rating of 6.0 (SD 0.6). Neutral pictures included buildings, plants, furniture etc., with a mean valence rating of 5.1 (SD 0.3) and arousal rating of 3.0 (SD 0.4). Mean valence and arousal ratings are based on IAPS normative ratings. ${ }^{37}$ Pictures were selected in such a way that negative and positive pictures were significantly more arousing than the neutral stimuli, based on the IAPS normative arousal ratings ( $t=39.0$ and 31.2 for negative and positive respectively, $P<0.001)$. Negative pictures were slightly more arousing than positive pictures $(t=-$ $4.3, P<0.001)$. For a list of the selected IAPS pictures, see Supplementary Table 1. 


\section{Functional MRI paradigm}

The experimental task was programmed using Presentation ${ }^{\circledR} 16.3$ (Neurobehavioral Systems, Inc.). Stimuli were presented on a 30 inch shielded LCD monitor $(60 \mathrm{~Hz})$ at a distance of $245 \mathrm{~cm}$ and were seen via a mirror system installed on top of the head coil. Vision correction was applied when necessary. Participants were instructed to passively view the 195 colored IAPS images during scanning. An event-related design was chosen since the arousal response is sensitive to habituation and stimulus predictability, and may actually decrease (instead of building up) in case of consecutively presented pictures of the same or similar intensity, as in a block design. Moreover, in order to avoid long-lasting mood states the pictures were presented in a randomized order, with no more than two pictures of the same valence or arousal level in a row. Thirty 'null-events' (i.e., black screen with a white fixation cross) were added to the paradigm, leading effectively to variable stimulusonset asynchronies.

Figure 1 represents a schematic illustration of the experimental design. The experiment consisted of one session that lasted for approximately 25 minutes. Each trial started with a black screen with a white fixation cross presented for 2500 ms, followed by a picture or null-event that was presented for $3000 \mathrm{~ms}$. After an interstimulus interval of $500 \mathrm{~ms}$ a prompt appeared on the screen for $2000 \mathrm{~ms}$ asking the participants whether the pictured contained a (part of a) human. They were instructed to answer by pressing with the index and middle finger of their dominant/operating hand corresponding to the left/right position of the 'human/non-human' word on the screen. This task was included in order to control for attention without referring to the emotional content of the picture. The error rates for this task were merely used for observatory purposes and not included in the analyses.

The onsets of both the pictures and the prompts were jittered in order to achieve optimal sampling of the hemodynamic response. ${ }^{39} \mathrm{~A}$ practice session was held prior to the $\mathrm{fMRI}$ experiment and outside the scanner, including 7 pictures from each valence category alternated by the prompt. Again, participants were asked to answer with a self-paced button press. The practice task followed the same script as the fMRI task, yet no jittering was added.

\section{Post-scanning subjective ratings}

Directly after fMRI scanning, participants viewed the same set of pictures on a computer outside the scanner. The individual order of pictures was kept identical to 


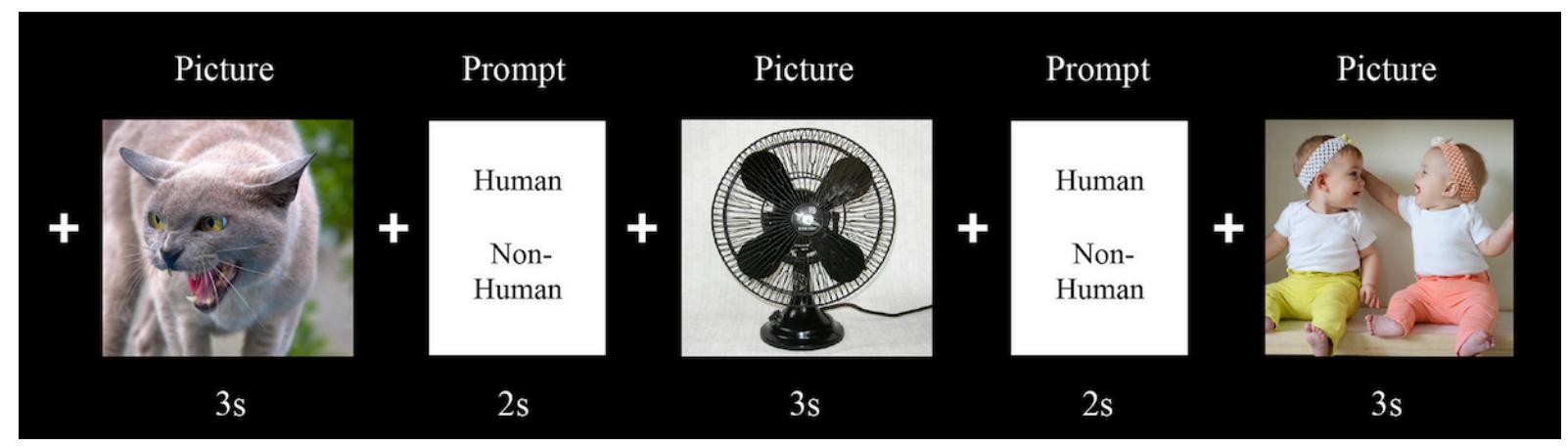

Figure 1. Experimental design of the event-related emotional processing task. Each trial started with a black screen with a white fixation cross, followed by a picture or null-event (fixation cross). After an inter-stimulus interval (fixation cross) a prompt appeared on the screen asking the participants whether the pictured contained a (part of a) human or not. The onsets of both the pictures and prompt were jittered in order to achieve optimal sampling of the hemodynamic response. Note that the pictures depicted in Figure 1 are not from the IAPS. In the actual experiment the words human/nonhuman were placed next to each other.

the order during the fMRI experiment. Participants were asked to rate every picture along the dimensions of valence and arousal using two independent ordinal scales from a paper-and-pencil version of the Self-Assessment Manikin (SAM). ${ }^{40}$ The scales ranged from negative (1) to neutral to positive (9) for valence, and from calm (1) to highly arousing (9) for arousal. The post-scanning image ratings were deliberately held outside the scanner, as the emotional evaluation of pictures requires explicit cognitive processes, which may suppress or alter implicit functional emotional reactivity. ${ }^{41,42}$

\section{fMRI data acquisition}

The images were acquired with a 3-T TRIO MRI system (Siemens, Erlangen, Germany) using a T2*-weighted EPI sequence (repetition time $=2200 \mathrm{~ms}$, echo time $=30 \mathrm{~ms}$ ). In total, 810 images were acquired, each of which consisted of 36 axial slices with a thickness of $3.1 \mathrm{~mm}$ (flip angle $=90^{\circ}$, distance factor $=10 \%$, field of view $(F O V)=200 \mathrm{~mm}, 64 \times 64$ matrix resulting in a voxel size of $3.1 \times 3.1 \times 3.0$ $\mathrm{mm}^{2}$ ). The slices covered the whole brain and were acquired parallel to the anteriorposterior commissure line. High-resolution, anatomical images (voxel size $1 \times 1 \times 1$ $\mathrm{mm}^{3}$ ) were acquired for anatomical reference using a standard T1-weighted 3D MP. RAGE sequence.

\section{Behavioral data analysis}

Statistical analyses were performed with the Statistical Package for Social Sciences (SPSS, version 21). The chi-square technique was used to compare proportions. 
Demographic and clinical data were compared with $t$-tests/ANOVA or Mann-Whitney $\mathrm{U} /$ Friedman's ANOVA test, whenever homogeneity of variances was violated $(P<0.05 ; 2$ tailed).

For the subjective ratings of valence and arousal, within group differences were calculated using paired $t$-tests. Comparisons between groups were performed using multivariate ANOVAs for valence and arousal separately, with picture category (positive/neutral/negative valence; positive high arousal/neutral low arousal/negative high arousal) as dependent variable, and group as independent or between group variable. Pillai's Trace $F$ approximations are reported. The Bonferroni correction was used to correct for multiple comparisons where necessary. Correlation analyses were conducted to investigate associations between clinical scores, task performance, and the BOLD signal in the maximally activated voxels in the significant clusters.

\section{fMRI data analysis}

The fMRI data were analyzed using the Statistical Parametric Mapping (SPM) Program (Wellcome Department of Imaging Neuroscience, London, United Kingdom www.fil.ion.ucl.ac.uk/spm/software/spm8). The first nine images were excluded from the analysis as these were acquired within the time period the MR signal needs to reach a steady state. All images were first spatially realigned to correct for inter-scan movement. Then the mean EPI image for each subject was computed and spatially normalized to the Montreal Neurological Institute (MNI) template using the "unified segmentation" function. ${ }^{43}$ The data were then smoothed using a Gaussian kernel (full width at half maximum of $8 \mathrm{~mm}$ ).

Overall, three onset regressors were defined, one for the negative, one for the neutral, and one for the positive pictures, indicating the onset times of individual trials (each considered as a single event). For each onset regressor, we included one additional regressor into the design matrix with parametric modulations representing the individual subjective rating of arousal (including parametric modulation). The hemodynamic response to each event type was modeled using a canonical synthetic hemodynamic response function (HRF) and its first derivative. The six head movement parameters were included as confounds.

\section{Within Group Comparisons}

First-level linear baseline contrasts were calculated comparing each regressor with the implicit baseline (i.e., those time periods that were not explicitly modeled and 
those during which no event occurred) by setting the regressors of interest to 1 and all other regressors to zero. These contrasts were then taken to the second level where they were subjected to within-subject analysis of variance (ANOVA, flexible factorial design in SPM8) with a single factor condition (positive, neutral, negative) using a family-wise error corrected threshold of $P_{\mathrm{FWE}}<0.05$ at the voxel-level.

\section{Between Group Comparisons}

In order to identify the main effect of the experimental task, first level linear contrasts were calculated, comparing events of the different conditions (e.g., negative>neutral, positive>neutral etc.). These contrasts were taken to the second level where they were subjected to two-sample $t$ tests (between group) again using a family-wise error corrected threshold of $P_{\mathrm{FWE}}<0.05$ at the voxel-level.

In addition to the analyses at the whole brain level, region-of-interest (ROI) between group analyses were performed following differences in the BOLD signal during implicit emotional processing in the current experiment between PD patients and controls subjects as triggered by the IAPS pictures. As such, we used previous imaging studies on emotional processing in PD patients ${ }^{19}$ and healthy volunteers ${ }^{44}$ to identify the coordinates for the bilateral putamen (left putamen: $-24,-3,+3$; right putamen: $+24,+6,-6)^{19}$ and the right medial PFC $(+3,+54,+27)^{44}$ as ROls. The ROI analyses of the BOLD signal revealed differences between PD patients and control subjects during implicit emotional processing triggered by the IAPS pictures. For the ROI analyses, significant activations are reported at $P_{\text {svc }}<0.05$ (small volume correction, i.e., family-wise error correction within the search volume). Beta values representing estimates of BOLD signal amplitudes were extracted for the maximally activated voxels within the significant clusters revealed by the ROI analyses.

\section{Results}

\section{Behavioural results}

Valence and arousal ratings for PD patients and controls are presented in Table 2. In both groups, positive/negative pictures were considered to be more positive/negative (PD: $t(18)=15.38 /-21.74, P<0.001$; Controls: $t(18)=12.76 /-18.15, P<0.001)$ and more arousing (PD: $t(18)=18.25 / 13.13, P<0.001$; Controls: $t(18)=8.33 / 9.29, P<0.001)$ than neutral pictures. Also, both groups considered negative pictures to be more negative (PD: $t(18)=-21.12, P<0.001$; Controls: $t(18)=-17.43, P<0.001)$ and more arousing (PD: $t(18)=8.42, P<0.001$; Controls: $t(18)=3.66, P<0.001)$ than positive pictures. All 
pairwise comparisons were corrected for multiple comparisons (Bonferroni). PD patients and controls did not show significant between group differences for both valence and arousal ratings.

In PD patients, valence ratings for positive and neutral pictures were negatively correlated with levels of persistent anxiety (i.e., PAS persistent; $r=-.49$ /$.61, P=0.033 / 0.005$ respectively). In addition, neutral valence ratings were negatively correlated with general and episodic levels of anxiety (i.e., PAS total and episodic; $r=-.63 /-.54, P=0.004 / 0.018$ respectively) and clinically relevant levels of apathy (i.e., Apathy Criteria; $r=-.46, P=0.048$ ). Arousal ratings for negative pictures were positively correlated with levels of anxiety (i.e., HARS; $r=.47, P=0.043$,), positive arousal ratings were positively associated with executive functioning (i.e., $\mathrm{FAB} ; r=.56, P=0.013$ ), and neutral or low arousal ratings were negatively correlated with levels of persistent anxiety (i.e., PAS persistent; $r=-.46, P=0.046$ ). In controls, valence ratings for negative pictures were negatively correlated with anxiety scores (i.e., HARS; $r=-.53, P=0.019$ ) and negative high arousal ratings showed a negative correlation with episodic levels of anxiety (i.e., PAS episodic; $r=$ $.47, P=0.041$ ).

Table 2. Subjective valence and arousal ratings for Parkinson patients $(n=19)$ and healthy controls $(n=19)$

\begin{tabular}{lrrrr}
\hline & Positive/high & Neutral/low & Negative/high & $P$ - value \\
Valence ratings & & & & \\
PD & $6.8(0.7)$ & $5.3(0.3)$ & $2.2(0.4)$ & $<0.001^{*}$ \\
Controls & $7.0(0.8)$ & $5.4(0.4)$ & $2.2(0.6)$ & $<0.001^{*}$ \\
PD vs. Controls & $\mathrm{ns}$ & $\mathrm{ns}$ & $\mathrm{ns}$ & \\
Arousal ratings & & & & \\
PD & $4.7(1.2)$ & $2.1(0.8)$ & $6.9(1.1)$ & $<0.001^{*}$ \\
Controls & $4.6(1.7)$ & $2.1(1.1)$ & $6.1(1.6)$ & $<0.001^{*}$ \\
PD vs. Controls & $\mathrm{ns}$ & $\mathrm{ns}$ & $\mathrm{ns}$ & \\
\hline
\end{tabular}

Note: Depicted are means and standard deviations. The 0-9 SAM Scale was used: valence ratings, 0 is most negative and 9 is most positive; arousal ratings, 0 is lowest arousal and 9 is highest arousal. ns: no significant group difference. *Significant within-group differences between conditions. 


\section{BOLD functional MRI results}

Within group differences

Multiple significant within group differences of activated brain regions and structures were found in PD patients and controls for the different emotional categories (all $P_{\mathrm{FWE}}<0.05$, family-wise error corrected, see Supplementary Tables 2 and 3). Here, we only present within group differences when comparing pictures depicting high levels of arousal (i.e., positive and negative collapsed) with those depicting low levels of arousal (i.e., high>low). PD patients and controls both showed increased activity in the left and right middle temporal gyrus (MTG), left and right fusiform gyrus, left and right middle occipital gyrus (MOG), left OFC, and left and right vIPFC. However, controls showed more pronounced activity in the left thalamus and the brainstem, while PD patients showed more pronounced activity in the right OFC, and left and right dorsomedial prefrontal cortex (dmPFC). For the opposite contrast (i.e., low>high), controls only showed increased activity in the left fusiform gyrus. For within group differences for all emotional categories, see Supplementary Table 2 and 3.

\section{Between group differences}

At the predefined whole brain threshold of $P_{\mathrm{FWE}}<0.05$ (family-wise error correction at the voxel-level), there were no significant between-group differences. However, Table 3 summarizes the significant differences in brain activation between PD patients and controls for the modulation of valence and arousal as revealed by the ROI-analyses $\left(P_{\mathrm{svc}}<0.05\right.$, small volume correction/ region-of-interest [ROI], i.e., family-wise error correction within the search volume/ROI).

For stimuli with positive valence, PD patients (compared to controls) showed increased activity in the dmPFC, while controls (compared to patients) showed increased activity in the left putamen (both $P_{\text {svc }}<0.05$, see Table 3 ). With respect to the analysis of the factor arousal, negative and positive pictures were collapsed as high arousing pictures, while neutral pictures served as low arousing pictures. During the (implicit) processing of both the low and high arousing pictures, controls showed differential activity in the left (and right) posterior putamen when compared to PD patients $\left(P_{\mathrm{svc}}<0.05\right.$, see Figure 2 and Table 3$)$. However, close inspection of the activated clusters (i.e., the BOLD signal changes as indexed by the beta values of the most active voxels) revealed that left (posterior) putamen activity was reduced in 
PD patients, as reflected by the negative beta values. Moreover, although the reduced activity was found for both low and high arousing stimuli, it appeared to be most pronounced for high arousing stimuli (Supplementary Fig. 1a).

In contrast to controls, PD patients showed differential activity in the right dmPFC ( $P_{\mathrm{svc}}<0.05$, see Figure 2 and Table 3$)$ when processing low and high arousing pictures. The beta values of the most active voxels within the dmPFC clusters indexing the BOLD signal changes during the processing of low and high arousing stimuli revealed increased right dmPFC activity in PD patients, but also a pronounced decrease of right dmPFC activity in controls, again the difference between PD patients and controls was most prominent for the high arousing stimuli (Supplementary Fig. 1b).

All between group differences remained significant after correction for symptoms of apathy, depression, anxiety, and use of antidepressants. Neural activation patterns in patients were also not significantly influenced by the use of levodopa, disease duration, and severity of motor symptoms.

Table 3. Functional differences between Parkinson patients (PD) and healthy controls (HC) as revealed by region-of-interest analyses $(\mathrm{ROI})$

\begin{tabular}{|c|c|c|c|c|c|c|c|}
\hline \multirow{2}{*}{$\begin{array}{l}\text { Contrasts } \\
\text { VALENCE }\end{array}$} & \multirow[t]{2}{*}{ Brain region } & \multirow[t]{2}{*}{ Cluster size } & \multicolumn{3}{|c|}{$\begin{array}{c}\text { MNI Coordinates } \\
x / y / z\end{array}$} & \multirow[t]{2}{*}{$\mathbf{T}$} & \multirow[t]{2}{*}{$P_{\text {value }}$} \\
\hline & & & & & & & \\
\hline \multicolumn{8}{|l|}{ Positive } \\
\hline $\mathrm{HC}>\mathrm{PD}$ & L Putamen & 21 & -24 & -6 & 10 & 3.81 & .010 \\
\hline $\mathrm{PD}>\mathrm{HC}$ & R Dorsomedial prefrontal cortex & 177 & 4 & 62 & 20 & 5.25 & .002 \\
\hline \multicolumn{8}{|l|}{ AROUSAL } \\
\hline \multicolumn{8}{|l|}{ Low arousal } \\
\hline $\mathrm{HC}>\mathrm{PD}$ & L Putamen & 201 & -20 & 10 & -2 & 4.23 & .018 \\
\hline $\mathrm{PD}>\mathrm{HC}$ & R Dorsomedial prefrontal cortex & 75 & 8 & 62 & 24 & 4.10 & .022 \\
\hline \multicolumn{8}{|l|}{ High Arousal } \\
\hline \multirow[t]{2}{*}{$\mathrm{HC}>\mathrm{PD}$} & L Putamen & 131 & -24 & -6 & 10 & 4.49 & .002 \\
\hline & R Putamen & 122 & 24 & 10 & 2 & 4.06 & .008 \\
\hline $\mathrm{PD}>\mathrm{HC}$ & R Dorsomedial prefrontal cortex & 104 & 8 & 64 & 26 & 4.63 & .009 \\
\hline
\end{tabular}

Cluster size denotes the extent of the activation cluster by number of significant voxels $\left(\mathrm{k}_{\mathrm{E}}\right)$. MNI coordinates refer to the location of the maximally activated voxel (peak) within an activation cluster. Results are considered significant at $P_{\mathrm{svc}}<0.05$ (FWE-corrected for small volume/ region-of-interest). Note: high arousal means positive and negative pictures collapsed. Low arousing stimuli are equivalent to neutral pictures. 
A) HC > PD Low Arousal

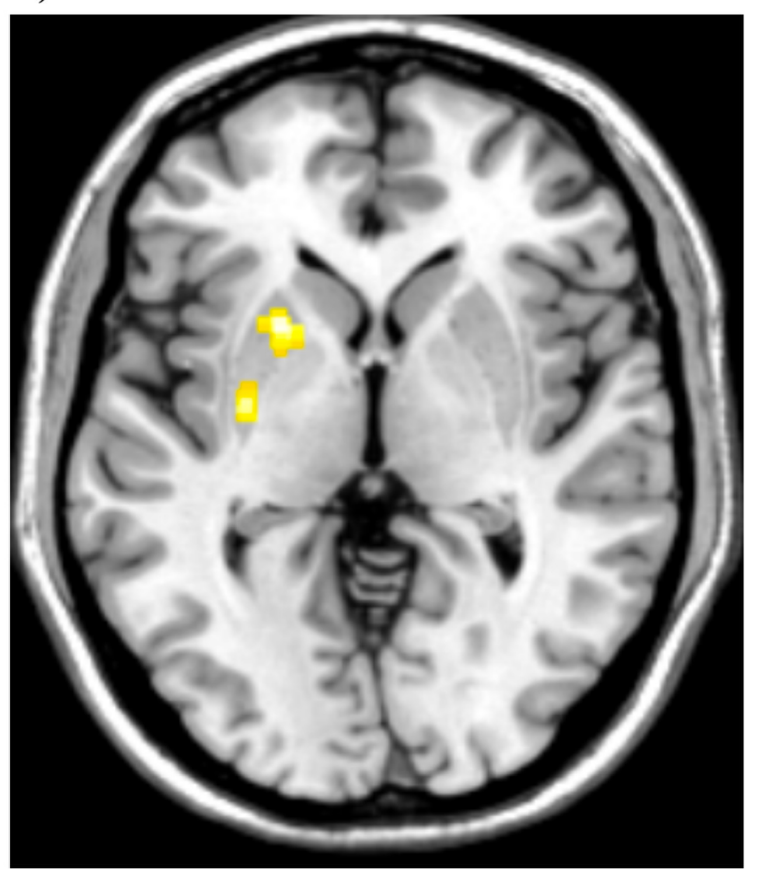

C) PD > HC Low Arousal

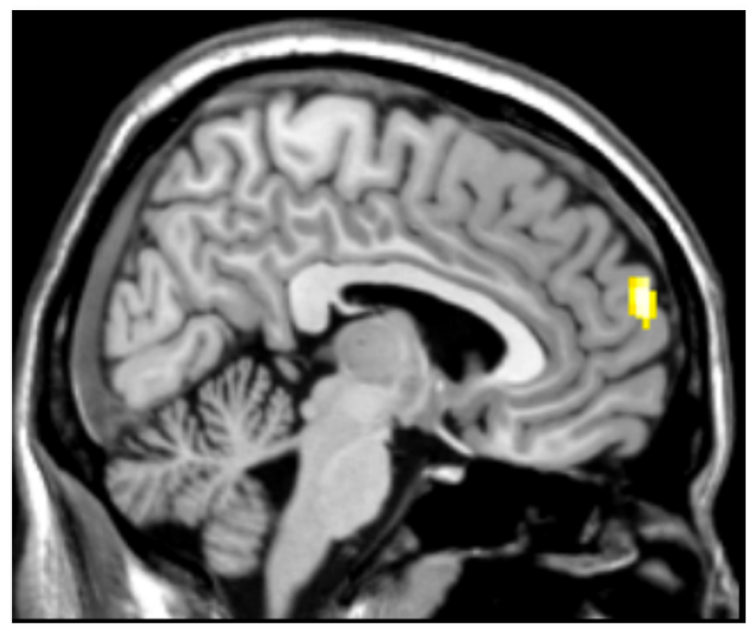

\section{B) HC > PD High Arousal}

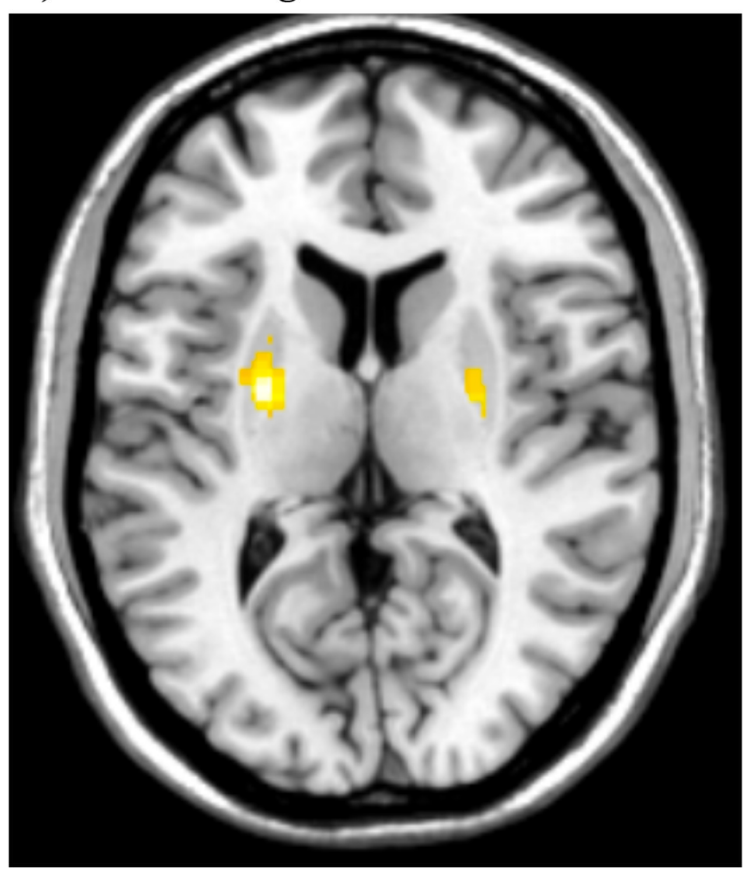

D)PD > HC High Arousal

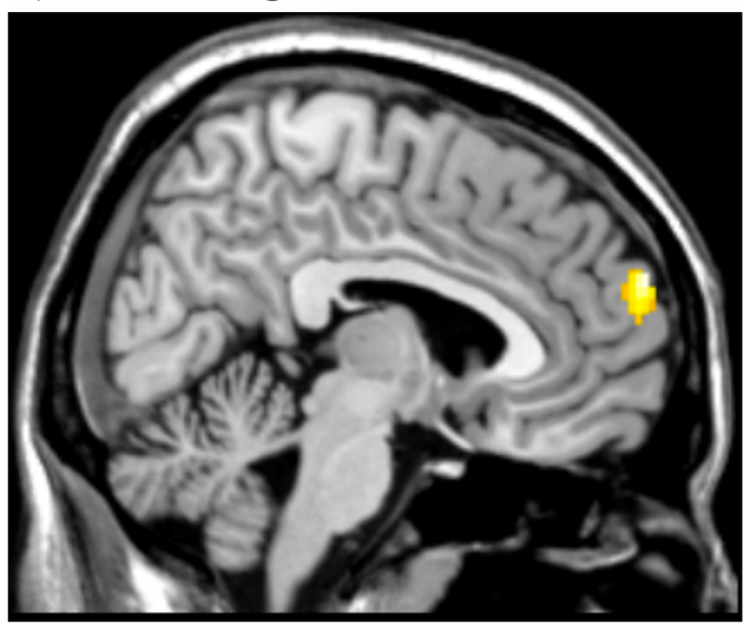

$P<.05$

Figure 2. Results of the region-of-interest (ROI) analysis on the processing of low and highly arousing stimuli in PD patients and healthy controls $(\mathrm{HC})$. Contrasting $\mathrm{HC}$ with PD patients showed a differential increase of BOLD signal for $\mathrm{HC}$ in the left (posterior) putamen for low arousal $(\mathbf{A})$ and bilaterally in the putamen for high arousal $\left(\mathbf{B}\right.$, all $\left.P_{\mathrm{SvC}}<0.05\right)$. Contrasting PD patients with $\mathrm{HC}$ revealed an increased BOLD signal for $\mathrm{PD}$ patients in the right dorsomedial prefrontal cortex (dmPFC) for low (C) arousal and high arousal (D, all $\left.P_{\mathrm{svc}}<0.05\right)$. 
Correlation analyses of BOLD fMRI data with behavioral and clinical data In PD patients, higher scores for avoidance anxiety (PAS) were positively correlated with lower left (posterior) putamen activity (i.e., more negative beta values) for low arousing stimuli and positive stimuli ( $r=.52 / r=.53, P=0.02$, respectively), and with lower right (posterior) putamen activity (i.e., more negative beta values) for highly arousing stimuli $(r=-.48, P=0.04)$. Higher valence ratings for both neutral and positive pictures were correlated with increased activity in the right dmPFC for highly arousing stimuli ( $r=.61 / r=.57, P<0.01$, respectively). In contrast, higher scores for total anxiety, persistent anxiety and episodic anxiety (PAS) were inversely correlated with decreased right dmPFC activity for highly arousing stimuli $(r=-.65 / r=-.58 / r=-$ $.72, P<0.01$, respectively), as was a higher apathy score (LARS dichotomized) $(r=-$ $.47, P=0.042)$. Also, higher episodic anxiety scores in PD patients were correlated with increased right dmPFC activity (i.e., less negative beta values) for low arousing neutral stimuli $(r=-.47, P=0.041)$. Higher arousal ratings for neutral pictures were correlated with increased right dmPFC activity for positive pictures $(r=-.50, P=$ 0.03). Healthy controls showed an inverse correlation between lower left posterior putamen activity and higher scores for cognition (MMSE) for low arousing stimuli ( $r=$ -.64, $P<0.01)$ and higher scores for executive function $(F A B)$ and episodic anxiety (PAS) for positive stimuli $(r=-.63 / r=-.56, P=0.004 / 0.013$, respectively).

\section{Discussion}

We investigated the neurobiology of emotional processing in Parkinson's disease (PD) with event-related BOLD-fMRI in patients with mild to moderate PD and matched healthy controls during a standardized implicit emotional processing task. PD patients were capable of explicitly discriminating and rating the intensity of emotions after the scanning, as shown by similar stimuli ratings as controls. However, PD patients showed reduced functional activity of the left (and right) posterior putamen, and an increase of right-sided activity in the dorsal medial frontal regions (i.e., the right dmPFC), both most pronounced in response to highly arousing emotional stimuli. These results are in line with prior neuroimaging studies in PD on emotional processing that showed an intact ability to recognize and categorize emotions in the presence of frontal-subcortical limbic circuitry abnormalities. ${ }^{7,8,10}$ Importantly, our findings extend previous work by revealing for the first time a possible compensatory neural mechanism in PD patients, with an increased medial 
frontal activity that may compensate for striatal dysfunction during emotional processing.

Several explanations are possible for the reduced (bilateral) posterior putaminal activity in PD patients. The patients in this study were all examined under dopamine replacement therapy (DRT), with the exception of one patient who did not receive any antiparkinsonian drugs. Whereas DRT can improve depleted dopamine levels in the striatum for the benefit of improving motor symptoms, the effect on nonmotor functions such as reversal learning, reward-based decision-making, and emotional processing may be adverse when mesolimbic dopaminergic projections are still relatively intact. ${ }^{45-47}$ Animal lesion studies demonstrate dopamine D1 receptor super-sensitivity due to denervation of dopamine neurons in the substantia nigra. ${ }^{48}$ As the putamen is thought to have the highest density of dopamine D1 receptors in the human brain, ${ }^{49}$ the use of dopamine agonists may improve motor symptoms on one hand, but possibly prevents this structure from normal functioning during nonmotor processes such as emotional reactivity. Although we did not find a direct relation between the use of DRT in general or the use of dopamine agonists and reduced (posterior) putaminal activity in our patient sample, we did find a significant association between higher levels of (subclinical) social phobia (i.e., PAS avoidance subscale) and decreased (posterior) putaminal activity in PD patients. This finding suggests that reduced activity in the putamen may be associated with early disturbances in emotion regulation (i.e., increased anxiety) in these patients.

Another explanation, and one that we consider relevant, is more directly related to the pathology of PD. Several studies have shown that putaminal volume is significantly reduced in PD patients, and exhibits increasing atrophy as the disease progresses. $^{50,51}$ Resting state functional connectivity research showed reduced correlations of striatal activity with activity in the thalamus, midbrain, pons, and brainstem in PD patients, most pronounced for the posterior putamen. ${ }^{52}$ Also, loss of dopaminergic neurons in the substantia nigra subsequently results in reduced striatal dopamine transporter availability (DAT), amongst others in the putamen. ${ }^{19}$ From a theoretical point of view, Phillips and colleagues ${ }^{6}$ proposed a neurobiological framework of emotional processing where the putamen has strong connections with a ventral system of affective neurocircuitry that is particularly involved in the implicit perception of emotional stimuli and the generation of a physiological response. Considering the critical role of intact dopaminergic neurotransmission within this network, ${ }^{53}$ reduced putaminal DAT may disturb emotional reactivity remarkably. 
PD patients also showed an activity increase in the right dmPFC compared to controls, in particular for high arousing emotional stimuli. Together with the vmPFC and OFC, the dmPFC is part of the medial prefrontal network, which has been shown to play a significant role in the interaction between cognitive and emotional processes in the brain. ${ }^{54}$ Both the OFC and medial PFC have strong direct and indirect interconnections with subcortical limbic structures such as (subregions of) the amygdala, the ventral striatum, and autonomic brain regions such as the thalamus and brainstem. ${ }^{55-57}$ By means of feedforward and feedback projections, the medial prefrontal network is able to modulate (bottom-up) automatic processes arising from activated subcortical limbic regions by applying top-down cognitive control in order to regulate our emotions. ${ }^{6,58,59}$ An imbalance as reflected by either impaired cortical cognitive control or overactive subcortical bottom-up processes may contribute to several psychiatric disorders such as depression or anxiety. ${ }^{60}$ Considering the importance of intact dopaminergic neurotransmission for this emotional circuit to operate accurately, one may expect to see functional emotional processing deficits whenever dopamine supplies are reduced, as we see in PD. ${ }^{53}$

How can we explain this co-occurrence of reduced striatal activity and increased dmPFC activity in Parkinson patients? Several hypotheses about increased involvement of prefrontal structures in the elderly have emerged from brain imaging studies, such as the hemispheric asymmetry reduction in older age (HAROLD; ${ }^{61}$ ) and the compensation-related utilization of neural circuits hypothesis $\left(\mathrm{CRUNCH} ;{ }^{62}\right)$. According to $\mathrm{CRUNCH}$, older adults' brains are capable of addressing alternative neural resources in order to compensate for functional decline elsewhere in the brain. Especially at lower levels of task demand where executive control functions are required for processes as attention selection or context processing, increased activation is observed in regions of the prefrontal cortex. Although this hypothesis focuses mainly on differences in functional activity between older and younger adults, the same principle of compensational neural activity may be applicable to functional decline due to neurodegenerative brain disease. Findings on functional connectivity between prefrontal and subcortical regions support the view of a direct excitatory influence of the dmPFC on both amygdalar and autonomic brain regions. ${ }^{55,56,63}$ The increased activity in the right dmPFC in our PD sample may therefore have served as a compensatory (top-down) cognitive control mechanism, compensating for disturbed subcortical activity due to the loss of dopamine. 
This view is in line with our behavioral results showing that PD patients were unimpaired in rating both valence and arousal levels of pictures, which requires prefrontal cognitive control processes such as context processing and decisionmaking. In addition, we found that increased right dmPFC activity for intense emotional stimuli was associated with lower levels of anxiety in PD patients, which may provide another indication that the dmPFC is able the control subcortical limbic functioning. Nevertheless, in order to confirm our hypothesis on increased compensatory prefrontal (top-down) cognitive control mechanisms in PD patients, neuroimaging studies are warranted that measure functional connectivity patterns between frontal and subcortical limbic structures during emotional processing.

An alternative approach regarding the increased activity in dorsomedial prefrontal regions may involve the application of emotion regulation strategies. Although we instructed our participants to passively view the pictures in order to measure implicit processing of emotions, PD patients may have attempted to modulate their emotional response to high arousing stimuli and therewith reducing feelings of sadness, disgust, or sexual arousal. As the majority of PD patients in our study consistently reported that they have become more emotional since the onset of disease, it may be possible that the emotional content of the high arousing stimuli (both positive and negative) triggered them to neutralize their emotions, either consciously or unconsciously. In our PD sample, we only found a relation between anxiety scores and right dmPFC activity for high arousing stimuli (positive and negative collapsed) while this association was absent for intense positive stimuli only. This may suggest that the regulatory role of the right dmPFC is more involved in intense emotional stimuli with a negative value. Unfortunately, our BOLD signal for right dmPFC activity in response to negative stimuli only was not strong enough to confirm this hypothesis.

Both hypotheses indicate, though, that medial prefrontal cortex functioning may be an important target when treating affective disorders such as anxiety or depression. Recent functional imaging studies in patients without PD have shown that nonpharmacological treatments such as Cognitive Behavioral Therapy (CBT) can restore impaired neuronal affective processing by increasing functional connectivity between limbic and prefrontal cortices. ${ }^{64,65}$ Hence, PD patients who are cognitively intact may take advantage of the preserved and potential compensatory involvement of medial prefrontal cortices in emotion regulation, despite the neurobiological deficits in affective circuitry related to the pathology of PD. 
Therapeutic interventions, such as CBT, that rely on frontal cognitive control mechanisms or emotion regulation strategies may therefore be particularly useful when treating affective disorders in this specific population.

The following limitations of the present study need to be considered. All patients were assessed in their 'on' state and were taking stable doses of antiparkinsonian medication, with the exception of one patient without medication. As DRT can partially restore the loss of dopamine, and therewith improve functional reactivity, ${ }^{7,10}$ one might argue that from a methodological point of view it would be more informative to study patients off medication. However, in unmedicated PD patients, motor symptoms like tremor or dystonia can cause movement artifacts that may confound neuroimaging findings. Moreover, despite receiving DRT the patients in our sample still showed significant motor impairments (i.e., UPDRS-III), which indicates that the DRT did not fully compensate for the loss of dopamine. Furthermore, the main findings of the current study, i.e., differential neural activation patterns in PD patients, were robust against the effects of levodopa dose, disease duration, and severity of motor symptoms.

Another putative limitation concerns the use of a set of emotional and neutral stimuli (i.e., IAPS) that were normalized in a non-European sample, whereas all of our participants were of European origin and may have different standards when it comes to rating a picture as negative or positive. However, the inclusion of a large number of pictures of each category most likely ruled out this issue, as the behavioral analyses were based on group averages and not on ratings of individual pictures. This may, however, also explain why our fMRI results showed similar trends for high and low arousing stimuli. Both PD patients and control subjects tended to evaluate neutral pictures as slightly more positive compared to the normative IAPS ratings. Nonetheless, by calculating the beta values for each arousal category, we were able to reveal that the differences in functional activity between patients and controls were most pronounced for high arousing stimuli.

Finally, our additional attention check in which participants had to decide whether or not they saw a human in the picture may have elicited some frontal cognitive activation related to decision-making, and therewith possibly suppressed limbic area activity for instance in the amygdala. This, however, seems unlikely as most limbic regions were equally active in both patients and controls, except for the posterior putamen. 
In conclusion, our data on emotional processing showed decreased striatal reactivity in PD patients in response to intense emotional stimuli, without any deficit in actively discriminating and rating the intensity of emotions compared to matched healthy controls. We further observed increased prefrontal activation in right dorsomedial regions that may have served as a compensatory top-down cognitive control mechanism in two possible ways: i) to either restore dysfunction in subcortical limbic circuitry related to the pathology of the disease or ii) to modulate emotional responses for intense stimuli by the conscious or unconscious application of emotion regulation strategies. Both views can have important implications for the treatment of affective disorders in PD patients. Therapeutic interventions that rely on such cognitive control mechanisms, such as CBT, may be particularly useful for PD patients who are cognitively intact, as they can benefit from the intact or even compensatory influence of prefrontal areas in the treatment of affective disorders. 


\section{REFERENCES}

1. Chaudhuri KR, Healy DG, Schapira AHV. Non-Motor Symptoms of Parkinson's Disease: Diagnosis and Management. The Lancet Neurology 2006;5:235-245.

2. Martinez-Martin P, Rodriguez-Blazquez C, Kurtis MM, Chaudhuri K. The Impact of Non-Motor Symptoms on Health-Related Quality of Life of Patients with Parkinson's Disease. Mov Disord 2011;26:399-406.

3. Reijnders JSAM, Ehrt U, Weber WEJ, Aarsland D, Leentjens AFG. A Systematic Review of Prevalence Studies of Depression in Parkinson's Disease. Movement Disorders 2008;23:183-189.

4. Schrag A. Quality of Life and Depression in Parkinson's Disease. J Neurol Sci 2006;248:151-157.

5. Slawek J, Derejko M, Lass P. Factors Affecting the Quality of Life of Patients with Idiopathic Parkinson's Disease-a Cross-Sectional Study in an Outpatient Clinic Attendees. Parkinsonism Relat Disord 2005;11:465-468.

6. Phillips ML, Drevets WC, Rauch SL, Lane R. Neurobiology of Emotion Perception I: The Neural Basis of Normal Emotion Perception. Biological psychiatry 2003;54:504514.

7. Tessitore A, Hariri AR, Fera F, Smith WG, Chase TN, Hyde TM, et al. Dopamine Modulates the Response of the Human Amygdala: A Study in Parkinson's Disease. J Neurosci 2002;22:9099-9103.

8. Yoshimura N, Kawamura M, Masaoka Y, Homma I. The Amygdala of Patients with Parkinson's Disease Is Silent in Response to Fearful Facial Expressions. Neuroscience 2005;131:523-534.

9. Delaveau P, Salgado-Pineda P, Fossati P, Witjas T, Azulay JP, Blin O. Dopaminergic Modulation of the Default Mode Network in Parkinson's Disease. Eur Neuropsychopharmacol 2010;20:784-792.

10. Delaveau P, Salgado-Pineda P, Witjas T, Micallef-Roll J, Fakra E, Azulay JP, et al. Dopaminergic Modulation of Amygdala Activity During Emotion Recognition in Patients with Parkinson Disease. J Clin Psychopharmacol 2009;29:548-554.

11. Wieser MJ, Klupp E, Weyers P, Pauli P, Weise D, Zeller D, et al. Reduced Early Visual Emotion Discrimination as an Index of Diminished Emotion Processing in Parkinson's Disease? - Evidence from Event-Related Brain Potentials. Cortex 2012;48:1207-1217.

12. Miller KM, Okun MS, Marsiske M, Fennell EB, Bowers D. Startle Reflex Hyporeactivity in Parkinson's Disease: An Emotion-Specific or Arousal-Modulated Deficit? Neuropsychologia 2009;47:1917-1927.

13. Yuvaraj R, Murugappan M, Ibrahim NM, Sundaraj K, Omar MI, Mohamad K, et al. Inter-Hemispheric Eeg Coherence Analysis in Parkinson's Disease: Assessing Brain Activity During Emotion Processing. Journal of Neural Transmission 2014;1-16.

14. Yuvaraj R, Murugappan M, Ibrahim NM, Iqbal M, Sundaraj K, Mohamad K, et al. On the Analysis of Eeg Power, Frequency and Asymmetry in Parkinson's Disease During Emotion Processing. Behav. brain Funct 2014;10:12.

15. Wieser MJ, Muhlberger A, Alpers GW, Macht $M$, Ellgring $H$, Pauli $P$. Emotion Processing in Parkinson's Disease: Dissociation between Early Neuronal Processing and Explicit Ratings. Clin Neurophysiol 2006;117:94-102. 
16. Baggio HC, Segura B, Ibarretxe-Bilbao N, Valldeoriola F, Marti MJ, Compta Y, et al. Structural Correlates of Facial Emotion Recognition Deficits in Parkinson's Disease Patients. Neuropsychologia 2012;50:2121-2128.

17. Ibarretxe-Bilbao N, Junque C, Tolosa E, Marti MJ, Valldeoriola F, Bargallo N, et al. Neuroanatomical Correlates of Impaired Decision-Making and Facial Emotion Recognition in Early Parkinson's Disease. Eur J Neurosci 2009;30:1162-1171.

18. Delaveau P, Salgado-Pineda P, Micallef-Roll J, Blin O. Amygdala Activation Modulated by Levodopa During Emotional Recognition Processing in Healthy Volunteers: A Double-Blind, Placebo-Controlled Study. J Clin Psychopharmacol 2007;27:692-697.

19. Lotze M, Reimold M, Heymans U, Laihinen A, Patt M, Halsband U. Reduced Ventrolateral Fmri Response During Observation of Emotional Gestures Related to the Degree of Dopaminergic Impairment in Parkinson Disease. J Cogn Neurosci 2009;21:1321-1331.

20. Dietz J, Bradley MM, Jones J, Okun MS, Perlstein WM, Bowers D. The Late Positive Potential, Emotion and Apathy in Parkinson's Disease. Neuropsychologia 2013;51:960-966.

21. Schroder C, Mobes J, Schutze M, Szymanowski F, Nager W, Bangert M, et al. Perception of Emotional Speech in Parkinson's Disease. Mov Disord 2006;21:17741778.

22. Garrido-Vasquez P, Pell MD, Paulmann S, Strecker K, Schwarz J, Kotz SA. An ERP study of vocal emotion processing in asymmetric Parkinson's disease. Soc Cogn Affect Neurosci 2013; 8:918-927.

23. Yuvaraj R, Murugappan M, Ibrahim NM, Sundaraj K, Omar MI, Mohamad K, et al. Inter-Hemispheric Eeg Coherence Analysis in Parkinson's Disease: Assessing Brain Activity During Emotion Processing. Journal of Neural Transmission 2014a;1-16.

24. Yuvaraj R, Murugappan M, Ibrahim NM, Iqbal M, Sundaraj K, Mohamad K, et al. On the Analysis of Eeg Power, Frequency and Asymmetry in Parkinson's Disease During Emotion Processing. Behav. brain Funct 2014b;10:12.

25. De Rijk M, Rocca W, Anderson D, Melcon M, Breteler M, Maraganore D. A Population Perspective on Diagnostic Criteria for Parkinson's Disease. Neurology 1997;48:12771281.

26. Association AP. Diagnostic and Statistical Manual of Mental Disorders (Dsm). Washington, DC: American psychiatric association 1994;143-147.

27. Folstein MF, Folstein SE, McHugh PR. Mini-Mental State. A practical method for grading the cognitive state of patients for 1975;

28. Tomlinson CL, Stowe R, Patel S, Rick C, Gray R, Clarke CE. Systematic Review of Levodopa Dose Equivalency Reporting in Parkinson's Disease. Movement Disorders 2010;25:2649-2653.

29. Dubois B, Slachevsky A, Litvan I, Pillon B. The Fab a Frontal Assessment Battery at Bedside. Neurology 2000;55:1621-1626.

30. Hamilton M. A Rating Scale for Depression. Journal of neurology, neurosurgery, and psychiatry 1960;23:56.

31. Hamilton M. The Assessment of Anxiety States by Rating. British journal of medical psychology 1959;32:50-55. 
32. Leentjens AF, Dujardin K, Pontone GM, Starkstein SE, Weintraub D, Martinez-Martin P. The Parkinson Anxiety Scale (Pas): Development and Validation of a New Anxiety Scale. Movement Disorders 2014;29:1035-1043.

33. Sockeel P, Dujardin K, Devos D, Deneve C, Destée A, Defebvre L. The Lille Apathy Rating Scale (Lars), a New Instrument for Detecting and Quantifying Apathy: Validation in Parkinson's Disease. Journal of Neurology, Neurosurgery \& Psychiatry 2006;77:579-584.

34. Robert P, Onyike CU, Leentjens AFG, Dujardin K, Aalten P, Starkstein S, et al. Proposed Diagnostic Criteria for Apathy in Alzheimer's Disease and Other Neuropsychiatric Disorders. European Psychiatry 2009;24:98-104.

35. Hoehn MM, Yahr MD. Parkinsonism: Onset, Progression, and Mortality. Neurology 1998;50:318-318.

36. Fahn S, Jenner P, Marsden C, Teychenne P. Fahn S, Elton Ri, and Members of the Updrs Development Committee. The Unified Parkinson's Disease Rating Scale. Recent developments in Parkinson's disease. Florham Park, NJ: Macmillan Healthcare Information 1987.

37. Lang PJ, Bradley MM, Cuthbert BN. International Affective Picture System (laps): Affective Ratings of Pictures and Instruction Manual. Technical Report A-8 2008;

38. Brucke C, Kupsch A, Schneider GH, Hariz MI, Nuttin B, Kopp U, et al. The Subthalamic Region Is Activated During Valence-Related Emotional Processing in Patients with Parkinson's Disease. The European journal of neuroscience 2007;26:767-774.

39. Kehoe EG, Toomey JM, Balsters JH, Bokde ALW. Personality Modulates the Effects of Emotional Arousal and Valence on Brain Activation. Social cognitive and affective neuroscience 2012;7:858-870.

40. Bradley MM, Lang PJ. Measuring Emotion: The Self-Assessment Manikin and the Semantic Differential. Journal of behavior therapy and experimental psychiatry 1994;25:49-59.

41. Hariri AR, Bookheimer SY, Mazziotta JC. Modulating Emotional Responses: Effects of a Neocortical Network on the Limbic System. Neuroreport 2000;11:43-48.

42. Taylor SF, Phan KL, Decker LR, Liberzon I. Subjective Rating of Emotionally Salient Stimuli Modulates Neural Activity. Neuroimage 2003;18:650-659.

43. Ashburner J, Friston KJ. Unified Segmentation. Neuroimage 2005;26:839-851.

44. Phan KL, Taylor SF, Welsh RC, Decker LR, Noll DC, Nichols TE, et al. Activation of the Medial Prefrontal Cortex and Extended Amygdala by Individual Ratings of Emotional Arousal: A Fmri Study. Biological psychiatry 2003;53:211-215.

45. O'Callaghan $\mathrm{C}$, Bertoux $\mathrm{M}$, Hornberger $\mathrm{M}$. Beyond and Below the Cortex: The Contribution of Striatal Dysfunction to Cognition and Behaviour in Neurodegeneration. Journal of Neurology, Neurosurgery \& Psychiatry 2014;85:371-378.

46. Jellinger KA. The Pathology of Parkinson's Disease. Adv Neurol 2001;86:55-72.

47. Nestler EJ, Carlezon WA. The Mesolimbic Dopamine Reward Circuit in Depression. Biological psychiatry 2006;59:1151-1159.

48. Gerfen CR, Surmeier DJ. Modulation of Striatal Projection Systems by Dopamine. Annual review of neuroscience 2011;34:441.

49. Breese GR, Mueller RA, Celeste T, Neurobiology of Central D1-Dopamine Receptors (Springer Science \& Business Media, 2013), p. 197. 
50. Geng D-y, Li Y-X, Zee C-S. Magnetic Resonance Imaging-Based Volumetric Analysis of Basal Ganglia Nuclei and Substantia Nigra in Patients with Parkinson's Disease. Neurosurgery 2006;58:256-262.

51. Lisanby SH, McDonald WM, Massey EW, Doraiswamy PM, Rozear M, Boyko OB, et al. Diminished Subcortical Nuclei Volumes in Parkinson's Disease by Mr Imaging. Journal of neural transmission. Supplementum 1992;40:13-21.

52. Hacker C, Perlmutter J, Criswell S, Ances B, Snyder A. Resting State Functional Connectivity of the Striatum in Parkinson's Disease. Brain 2012;aws281.

53. Remy P, Doder M, Lees A, Turjanski N, Brooks D. Depression in Parkinson's Disease: Loss of Dopamine and Noradrenaline Innervation in the Limbic System. Brain 2005;128:1314-1322.

54. Ray RD, Zald DH. Anatomical Insights into the Interaction of Emotion and Cognition in the Prefrontal Cortex. Neuroscience \& Biobehavioral Reviews 2012;36:479-501.

55. Barbas H, Zikopoulos B. Sequential and Parallel Circuits for Emotional Processing in Primate Orbitofrontal Cortex. The orbitofrontal cortex 2006;1:57-93.

56. Price JL. Connections of Orbital Cortex. The orbitofrontal cortex 2006;39-55.

57. Ochsner KN, Gross JJ. The Neural Architecture of Emotion Regulation. Handbook of emotion regulation 2007;1:87-109.

58. Gross JJ. Emotion Regulation. Handbook of emotions 2008;3:497-513.

59. Gross JJ. Antecedent-and Response-Focused Emotion Regulation: Divergent Consequences for Experience, Expression, and Physiology. Journal of personality and social psychology 1998;74:224.

60. Rottenberg J, Gross JJ. When Emotion Goes Wrong: Realizing the Promise of Affective Science. Clinical Psychology: Science and Practice 2003;10:227-232.

61. Cabeza R. Hemispheric Asymmetry Reduction in Older Adults: The Harold Model. Psychology and aging 2002;17:85.

62. Reuter-Lorenz PA, Cappell KA. Neurocognitive Aging and the Compensation Hypothesis. Current directions in psychological science 2008;17:177-182.

63. Barbas H, Saha S, Rempel-Clower N, Ghashghaei T. Serial Pathways from Primate Prefrontal Cortex to Autonomic Areas May Influence Emotional Expression. BMC neuroscience 2003;4:25.

64. Okai D, Samuel M, Askey-Jones S, David AS, Brown RG. Impulse Control Disorders and Dopamine Dysregulation in Parkinson's Disease: A Broader Conceptual Framework. European Journal of Neurology 2011;18:1379-1383.

65. Mansson KN, Carlbring P, Frick A, Engman J, Olsson C-J, Bodlund O, et al. Altered Neural Correlates of Affective Processing after Internet-Delivered Cognitive Behavior Therapy for Social Anxiety Disorder. Psychiatry Research: Neuroimaging 2013;214:229-237. 
Supplementary Table 1. Pictures from the International Affective Picture System (IAPS) included in the present study.

\begin{tabular}{|c|c|c|}
\hline Neutral & Positive & Negative \\
\hline 2002 & 1650 & 1120 \\
\hline 2102 & 1710 & 1525 \\
\hline 2191 & 1811 & 1930 \\
\hline 2214 & 2045 & 2053 \\
\hline 2215 & 2058 & 2095 \\
\hline 2273 & 2071 & 2345.1 \\
\hline 2357 & 2075 & 2683 \\
\hline 2377 & 2216 & 2688 \\
\hline 2382 & 2345 & 2691 \\
\hline 2383 & 2347 & 2692 \\
\hline 2393 & 4220 & 2703 \\
\hline 2446 & 4520 & 2800 \\
\hline 2518 & 4597 & 2811 \\
\hline 2570 & 4598 & 2981 \\
\hline 2745.1 & 4599 & 3001 \\
\hline 2880 & 4607 & 3030 \\
\hline 2890 & 4608 & 3053 \\
\hline 5040 & 4623 & 3060 \\
\hline 5130 & 4626 & 3071 \\
\hline 5390 & 4660 & 3102 \\
\hline 5471 & 4676 & 3103 \\
\hline 5520 & 5260 & 3110 \\
\hline 5731 & 5450 & 3170 \\
\hline 5740 & 5470 & 3195 \\
\hline 6150 & 5480 & 3266 \\
\hline 7001 & 5621 & 3350 \\
\hline 7002 & 5623 & 3400 \\
\hline 7003 & 5626 & 3500 \\
\hline 7004 & 5629 & 6021 \\
\hline 7009 & 5700 & 6190 \\
\hline 7012 & 5825 & 6212 \\
\hline 7014 & 5833 & 6231 \\
\hline 7017 & 7220 & 6243 \\
\hline 7020 & 7230 & 6260 \\
\hline 7025 & 7270 & 6300 \\
\hline 7026 & 7330 & 6312 \\
\hline 7032 & 7405 & 6315 \\
\hline 7034 & 7451 & 6540 \\
\hline 7035 & 7502 & 6550 \\
\hline 7036 & 7650 & 6560 \\
\hline 7040 & 8030 & 6821 \\
\hline 7041 & 8034 & 6830 \\
\hline 7045 & 8080 & 9075 \\
\hline 7050 & 8090 & 9163 \\
\hline 7052 & 8158 & 9183 \\
\hline 7053 & 8161 & 9250 \\
\hline 7056 & 8163 & 9252 \\
\hline 7059 & 8170 & 9254 \\
\hline 7060 & 8178 & 9332 \\
\hline 7080 & 8179 & 9410 \\
\hline 7100 & 8180 & 9412 \\
\hline
\end{tabular}


CHAPTER 3 EMOTIONAL PROCESSING IN PARKINSON'S DISEASE: AN FMRI STUDY

\begin{tabular}{|l|l|l|}
\hline 7140 & 8185 & 9413 \\
\hline 7150 & 8186 & 9414 \\
\hline 7175 & 8190 & 9424 \\
\hline 7179 & 8210 & 9433 \\
\hline 7190 & 8370 & 9495 \\
\hline 7205 & 8380 & 9570 \\
\hline 7217 & 8420 & 9600 \\
\hline 7290 & 8470 & 9622 \\
\hline 7491 & 8490 & 9635.1 \\
\hline 7547 & 8496 & 9810 \\
\hline 7705 & 8499 & 9902 \\
\hline 7710 & 8501 & 9908 \\
\hline 7950 & 8502 & 9921 \\
\hline 8312 & 8531 & 9940 \\
\hline
\end{tabular}

The numbers refer to the pictures included in this study. 
Supplementary Table 2. Within group differences for PD patients $(n=19)$

\begin{tabular}{|c|c|c|c|c|c|c|}
\hline \multirow{2}{*}{$\begin{array}{l}\text { Contrasts } \\
\text { VALENCE }\end{array}$} & \multirow[t]{2}{*}{ Cluster size $\left(k_{E}\right)$} & \multicolumn{3}{|c|}{ MNI Coordinates $x / y / z$} & \multirow[t]{2}{*}{ T-value } & \multirow[t]{2}{*}{$P$-value } \\
\hline & & & & & & \\
\hline \multicolumn{7}{|l|}{ Positive > Neutral } \\
\hline R Middle temporal gyrus & 4851 & 52 & -54 & 4 & 8.87 & .000 \\
\hline $\begin{array}{l}\text { R Fusiform gyrus (posterior) } \\
\mathrm{R} \text { Inferior temporal/occipital } \\
\text { gyrus }\end{array}$ & & $\begin{array}{l}46 \\
50\end{array}$ & $\begin{array}{l}-48 \\
-74\end{array}$ & $\begin{array}{r}-26 \\
-4\end{array}$ & 8.78 & .000 \\
\hline $\begin{array}{l}\text { L Middle occipital gyrus } \\
\text { L Middle temporal gyrus } \\
\text { (posterior) } \\
\text { L Middle temporal gyrus }\end{array}$ & 3540 & $\begin{array}{l}-50 \\
-62 \\
-56\end{array}$ & $\begin{array}{l}-80 \\
-58 \\
-68\end{array}$ & $\begin{array}{l}6 \\
6\end{array}$ & $\begin{array}{l}5.94 \\
5.82\end{array}$ & $\begin{array}{l}.019 \\
.026\end{array}$ \\
\hline \multicolumn{7}{|l|}{ Neutral > Positive } \\
\hline \multicolumn{7}{|l|}{ No significant difference } \\
\hline \multicolumn{7}{|l|}{ Positive $>$ Negative } \\
\hline \multirow[t]{2}{*}{ R Precentral gyrus } & 5706 & 20 & -18 & 64 & 5.78 & .028 \\
\hline & & 14 & -32 & 56 & 5.71 & .034 \\
\hline R Superior frontal gyrus & & 14 & -2 & 60 & 5.70 & .034 \\
\hline \multicolumn{7}{|l|}{ Negative $>$ Positive } \\
\hline R Lateral Occipital cortex & 1366 & 46 & -72 & -6 & 5.90 & .021 \\
\hline R Fusiform gyrus (posterior) & & 44 & -46 & -24 & 5.71 & .034 \\
\hline L Lateral Occipital cortex & 1249 & -40 & -80 & -6 & 6.89 & .002 \\
\hline L Fusiform gyrus (posterior) & & -42 & -48 & -20 & 6.75 & .002 \\
\hline $\mathrm{R}$ Ventrolateral prefrontal cortex & 1039 & 52 & 30 & 10 & 6.88 & .002 \\
\hline $\mathrm{R}$ Ventrolateral prefrontal cortex & & 46 & 18 & 26 & 6.59 & .003 \\
\hline R Precentral gyrus & & 38 & 10 & 26 & 5.60 & .044 \\
\hline \multicolumn{7}{|l|}{ Neutral > Negative } \\
\hline L Precentral gyrus & 4034 & -10 & -20 & 56 & 6.27 & .008 \\
\hline L Postcentral gyrus & & -44 & -26 & 58 & 6.09 & .013 \\
\hline L Precentral gyrus & & -28 & -16 & 52 & 5.61 & .044 \\
\hline R Superior temporal gyrus & 2217 & 54 & 2 & 2 & 5.91 & .020 \\
\hline $\begin{array}{l}\text { R Superior temporal gyrus } \\
\text { (posterior) }\end{array}$ & & 62 & -22 & 0 & 5.84 & .025 \\
\hline L Superior temporal gyrus & 2008 & -48 & -4 & 8 & 5.89 & .022 \\
\hline $\begin{array}{l}\mathrm{L} \text { Superior temporal gyrus } \\
\text { (posterior) }\end{array}$ & & -48 & -32 & 12 & 5.81 & .026 \\
\hline L Planum temporale & & -66 & -12 & 0 & 5.73 & .032 \\
\hline R Inferior parietal lobule & 519 & 60 & -58 & 40 & 6.25 & .008 \\
\hline R Hippocampus & 321 & 22 & -42 & 10 & 5.56 & .049 \\
\hline L Middle occipital gyrus & 7620 & -50 & -78 & 8 & 12.59 & .000 \\
\hline L Fusiform gyrus & & -42 & -48 & -20 & 12.27 & .000 \\
\hline L Inferior occipital gyrus & & -40 & -80 & -6 & 11.68 & .000 \\
\hline
\end{tabular}


Supplementary Table 2 (continued). Within group differences for PD patients $(n=19)$

\begin{tabular}{|c|c|c|c|c|c|c|}
\hline \multirow{2}{*}{$\begin{array}{l}\text { Contrasts } \\
\text { VALENCE }\end{array}$} & \multirow[t]{2}{*}{ Cluster size $\left(\mathrm{k}_{\mathrm{E}}\right)$} & \multicolumn{3}{|c|}{ MNI Coordinates $x / y / z$} & \multirow[t]{2}{*}{ T-value } & \multirow[t]{2}{*}{$P$-value } \\
\hline & & & & & & \\
\hline \multicolumn{7}{|l|}{ Neutral > Negative } \\
\hline R Fusiform gyrus & 7040 & 44 & -48 & -24 & 14.38 & .000 \\
\hline R Inferior occipital gyrus & & 50 & -74 & -4 & 13.77 & .000 \\
\hline R Middle temporal gyrus & & 54 & -54 & 4 & 13.43 & .000 \\
\hline \multicolumn{7}{|l|}{ Negative $>$ Neutral } \\
\hline L Ventrolateral prefrontal cortex & 3209 & -44 & 22 & 18 & 7.16 & .001 \\
\hline L Dorsomedial prefrontal cortex & & -42 & 16 & 24 & 7.12 & .001 \\
\hline L Orbitofrontal cortex & & -28 & 32 & -20 & 6.35 & .006 \\
\hline R Dorsomedial prefrontal cortex & 3006 & 46 & 18 & 26 & 10.62 & .000 \\
\hline $\mathrm{R}$ Ventrolateral prefrontal cortex & & 52 & 32 & 10 & 8.43 & .000 \\
\hline $\mathrm{R}$ Inferior frontal gyrus & & 44 & 32 & 8 & 8.07 & .000 \\
\hline $\begin{array}{l}\text { R Superior dorsomedial } \\
\text { prefrontal cortex }\end{array}$ & 2700 & 6 & 52 & 22 & 7.87 & .000 \\
\hline $\begin{array}{l}\text { R Superior dorsomedial } \\
\text { prefrontal cortex }\end{array}$ & & 4 & 50 & 30 & 6.89 & .002 \\
\hline R Posterior cingulate gyrus & 1147 & 2 & -52 & 24 & 6.18 & .010 \\
\hline L Superior parietal lobule & 706 & -24 & -54 & 44 & 7.52 & .000 \\
\hline R Superior parietal lobule & 649 & 28 & -50 & 48 & 8.43 & .000 \\
\hline R Orbitofrontal cortex & 287 & 4 & 48 & -24 & 5.95 & .018 \\
\hline R Middle temporal gyrus & 229 & 54 & 0 & -24 & 6.17 & .011 \\
\hline R Brainstem & 202 & 8 & -28 & -8 & 5.68 & .037 \\
\hline \multicolumn{7}{|l|}{ AROUSAL } \\
\hline \multicolumn{7}{|l|}{ High Arousal > low arousal } \\
\hline L Middle occipital gyrus & 7351 & -50 & -78 & 8 & 12.00 & .000 \\
\hline L Fusiform gyrus & & -42 & -48 & -20 & 10.27 & .000 \\
\hline L Inferior occipital gyrus & & -42 & -80 & -4 & 9.63 & .000 \\
\hline R Fusiform gyrus & 7034 & 44 & -48 & -24 & 13.34 & .000 \\
\hline R Inferior occipital gyrus & & 50 & -74 & -4 & 12.93 & .000 \\
\hline R Middle temporal gyrus & & 54 & -54 & 4 & 12.85 & .000 \\
\hline R Posterior cingulate gyrus & 1789 & 2 & -52 & 24 & 5.92 & .020 \\
\hline R Dorsomedial prefrontal cortex & 1752 & 44 & 18 & 26 & 8.55 & .000 \\
\hline $\mathrm{R}$ Inferior frontal gyrus & & 44 & 32 & 8 & 6.28 & .008 \\
\hline $\mathrm{R}$ Ventrolateral prefrontal cortex & & 52 & 32 & 10 & 5.85 & .024 \\
\hline $\begin{array}{l}\text { R Superior dorsomedial } \\
\text { prefrontal cortex }\end{array}$ & 1677 & 6 & 56 & 20 & 6.22 & .009 \\
\hline L Dorsomedial prefrontal cortex & 1240 & -42 & 16 & 24 & 6.12 & .012 \\
\hline L Ventrolateral prefrontal cortex & & -44 & 22 & 18 & 5.67 & .038 \\
\hline L Superior parietal lobule & 817 & -24 & -54 & 44 & 7.03 & .001 \\
\hline
\end{tabular}


Supplementary Table 2 (continued). Within group differences for PD patients ( $n=19)$

\begin{tabular}{lcrrrrr}
\hline Contrasts & Cluster size $\left(\mathbf{k}_{\mathrm{E}}\right)$ & \multicolumn{1}{c}{ MNI Coordinates $\mathbf{x} / \mathbf{y} / \mathbf{z}$} & T-value & $\boldsymbol{P}$-value \\
\hline R Superior parietal lobule & 710 & 28 & -52 & 50 & 6.94 & .001 \\
R Orbitofrontal cortex & 387 & 4 & 48 & -22 & 5.67 & .038 \\
Low arousal > high arousal & & & & & & \\
No significant difference & & & & & & \\
\hline
\end{tabular}

Cluster size denotes the extent of the activation cluster by number of significant voxels $\left(k_{E}\right)$. $\mathrm{MNI}$ coordinates refer to the location of the maximally activated voxel (peak) within an activation cluster. Results are considered significant at $P<0.05$ (FWE corrected at the peak/voxel level).

Supplementary Table 3. Within group differences for Healthy Controls $(n=19)$

\begin{tabular}{|c|c|c|c|c|c|c|}
\hline \multirow{2}{*}{$\begin{array}{l}\text { Contrasts } \\
\text { VALENCE }\end{array}$} & \multirow[t]{2}{*}{ Cluster size $\left(k_{E}\right)$} & \multicolumn{3}{|c|}{ MNI Coordinates $x / y / z$} & \multirow[t]{2}{*}{ T-value } & \multirow[t]{2}{*}{$P$-value } \\
\hline & & & & & & \\
\hline \multicolumn{7}{|l|}{ Positive > Neutral } \\
\hline R Middle temporal gyrus & 3502 & 56 & -66 & 4 & 12.85 & .000 \\
\hline $\begin{array}{l}\text { R Fusiform gyrus } \\
\text { R Middle occipital/temporal } \\
\text { gyrus }\end{array}$ & & 44 & -44 & -22 & 7.18 & .025 \\
\hline L Middle occipital gyrus & 2421 & -50 & -76 & 4 & 11.66 & .000 \\
\hline L Fusiform gyrus & & -40 & -70 & -12 & 6.45 & .004 \\
\hline L Middle temporal gyrus & & -50 & -62 & 8 & 6.31 & .005 \\
\hline \multicolumn{7}{|l|}{ Neutral > Positive } \\
\hline \multicolumn{7}{|l|}{ No significant difference } \\
\hline \multicolumn{7}{|l|}{ Positive $>$ Negative } \\
\hline \multicolumn{7}{|l|}{ No significant difference } \\
\hline \multicolumn{7}{|l|}{ Negative > Positive } \\
\hline $\mathrm{R}$ Middle temporal gyrus & 2368 & 56 & -66 & 4 & 8.33 & .000 \\
\hline $\mathrm{R}$ Inferior temporal gyrus & & 46 & -52 & -18 & 6.91 & .001 \\
\hline R Fusiform gyrus & & 44 & -60 & -14 & 6.63 & .002 \\
\hline L Inferior occipital gyrus & 1965 & -50 & -78 & 4 & 7.00 & .001 \\
\hline L Fusiform gyrus & & -42 & -68 & -12 & 6.16 & .008 \\
\hline L Middle temporal gyrus & & -52 & -60 & 6 & 5.70 & .026 \\
\hline R Superior frontal gyrus & 978 & 8 & 56 & 40 & 6.17 & .008 \\
\hline $\mathrm{R}$ Ventrolateral prefrontal cortex & 678 & 56 & 30 & 14 & 8.33 & .000 \\
\hline \multicolumn{7}{|l|}{ Neutral > Negative } \\
\hline L Caudate Nucleus & 718 & -26 & -10 & 28 & 5.69 & .027 \\
\hline L Parahippocampal gyrus & 681 & -34 & -50 & 0 & 5.58 & .000 \\
\hline L Postcentral gyrus & 511 & -46 & -28 & 60 & 6.02 & .011 \\
\hline $\mathrm{R}$ Inferior parietal lobule & 291 & 54 & -64 & 42 & 5.81 & .020 \\
\hline
\end{tabular}


Supplementary Table 3 (continued). Within group differences for Healthy Controls $(n=19)$

\begin{tabular}{lcrrrrr}
\hline Negative > Neutral & & & & & & \\
R Middle temporal gyrus & 6599 & 56 & -66 & 4 & 21.18 & .000 \\
R Fusiform gyrus & & 44 & -50 & -18 & 13.30 & .000 \\
L Middle occipital gyrus & 6186 & -50 & -76 & 4 & 18.40 & .000 \\
L Fusiform gyrus & & -40 & -70 & -12 & 12.48 & .000 \\
L Middle temporal gyrus & & -50 & -60 & 8 & 11.97 & .000 \\
R Superior frontal gyrus & 3532 & 6 & 50 & 30 & 7.19 & .001 \\
R Superior medial frontal gyrus & & 8 & 44 & 40 & 6.64 & .002 \\
L Superior frontal gyrus & & -6 & 48 & 34 & 6.44 & .004 \\
L Orbitofrontal cortex & 2069 & -40 & 24 & -20 & 9.50 & .000 \\
L Ventrolateral prefrontal cortex & & -52 & 34 & 4 & 7.47 & .000 \\
R Ventrolateral prefrontal cortex & \multirow{2}{*}{1732} & 54 & 32 & 8 & 11.75 & .000 \\
R Inferior frontal gyrus & & 42 & 14 & 24 & 8.85 & .000 \\
R Posterior cingulate gyrus & \multirow{2}{*}{1723} & 2 & -52 & 30 & 7.04 & .001 \\
R Posterior parietal lobule & & 28 & -52 & 46 & 5.87 & .017 \\
R Amygdala & & 16 & -2 & -22 & 5.55 & .038 \\
Orbitofrontal cortex & \multirow{2}{*}{363} & 0 & 56 & -20 & 6.03 & .011 \\
L Orbitofrontal cortex & & -2 & 48 & -22 & 5.72 & .024 \\
\hline
\end{tabular}

AROUSAL

\begin{tabular}{lcrrrrr} 
High Arousal > Low Arousal & & & & & & \\
R Middle temporal gyrus & 5984 & 56 & -66 & 4 & 19.65 & .000 \\
R Fusiform gyrus & & 44 & -50 & -20 & 11.61 & .000 \\
R Middle occipital/temporal & & & & & & \\
gyrus & & 40 & -70 & 20 & 8.48 & .000 \\
L Middle occipital gyrus & 5621 & -50 & -76 & 4 & 17.36 & .000 \\
L Fusiform gyrus & & -40 & -70 & -12 & 10.93 & .000 \\
L Middle temporal gyrus & & -50 & -60 & 8 & 10.54 & .000 \\
L Superior frontal gyrus & 2049 & -10 & 50 & 36 & 5.59 & .034 \\
R Posterior cingulate gyrus & 1484 & 2 & -52 & 30 & 6.66 & .002 \\
Precuneus & & 0 & -50 & 48 & 5.61 & .032 \\
R Precuneus & & 8 & -52 & 14 & 5.61 & .033 \\
L Orbitofrontal cortex & 1282 & -40 & 24 & -20 & 7.68 & .000 \\
L Ventrolateral prefrontal cortex & & -52 & 32 & 4 & 5.78 & .021 \\
R Ventrolateral prefrontal cortex & 911 & 54 & 32 & 8 & 9.25 & .000 \\
L Thalamus & 400 & 16 & -28 & 0 & 5.63 & .031 \\
Low Arousal > High Arousal & & & & & & \\
\hline L Fusiform gyrus & 270 & -34 & -52 & 0 & 6.57 & .003 \\
\hline
\end{tabular}

Cluster size denotes the extent of the activation cluster by number of significant voxels $\left(\mathrm{k}_{\mathrm{E}}\right)$. MNI coordinates refer to the location of the maximally activated voxel (peak) within an activation cluster. Results are considered significant at $P<0.05$ (FWE corrected at the peak/voxel level). 
Supplementary Figure 1a. Mean beta values derived from the ROI analysis for the left posterior putamen in Parkinson patients (PD) and healthy controls (HC)

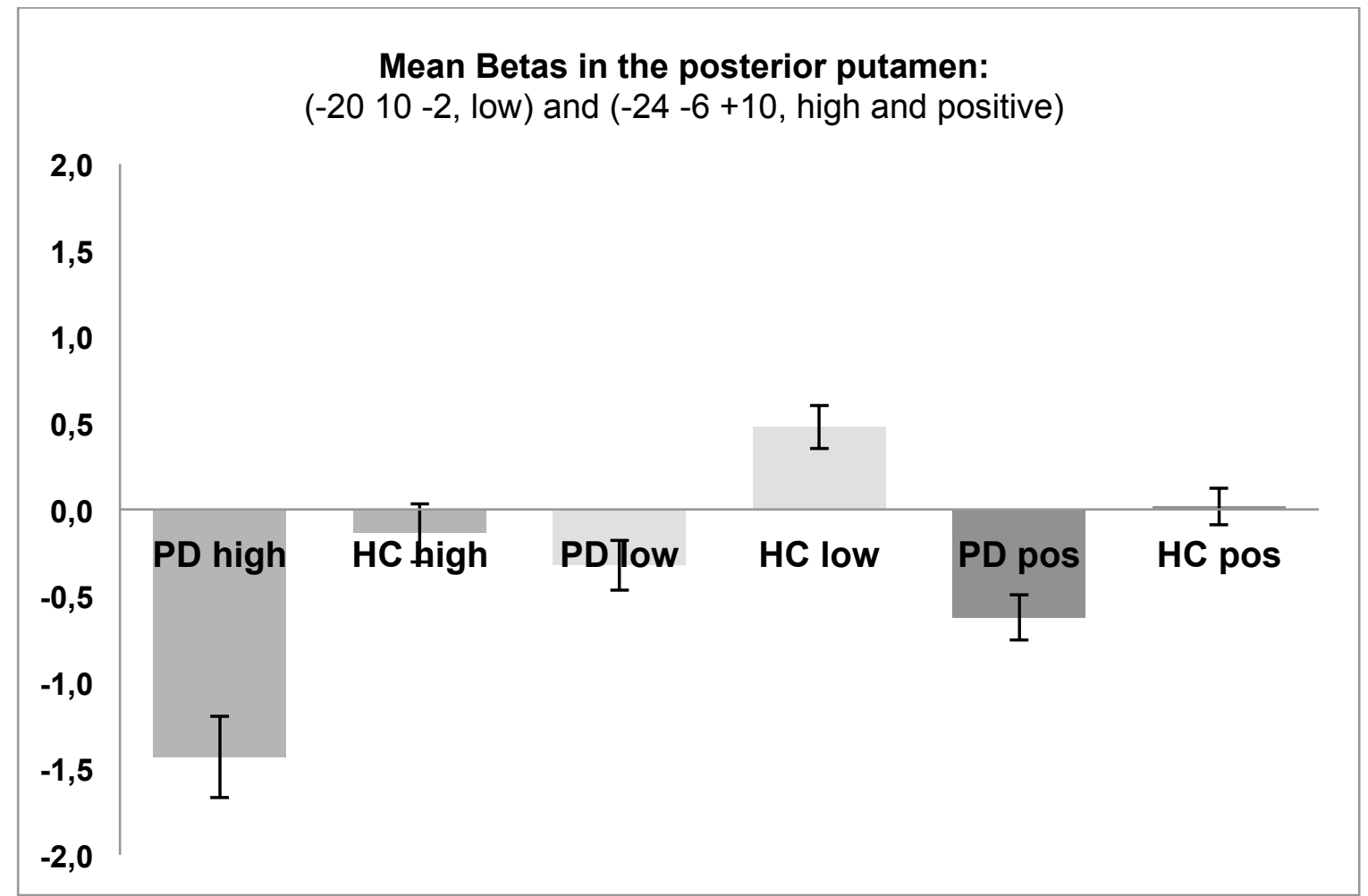

MNI coordinates refer to the location of the maxima. Results are considered significant at $P_{\mathrm{FWE}}<0.05$ small volume correction.

Supplementary Figure 1b. Mean beta values of ROI analyses right dorsomedial prefrontal cortex in Parkinson patients (PD) and healthy controls (HC)

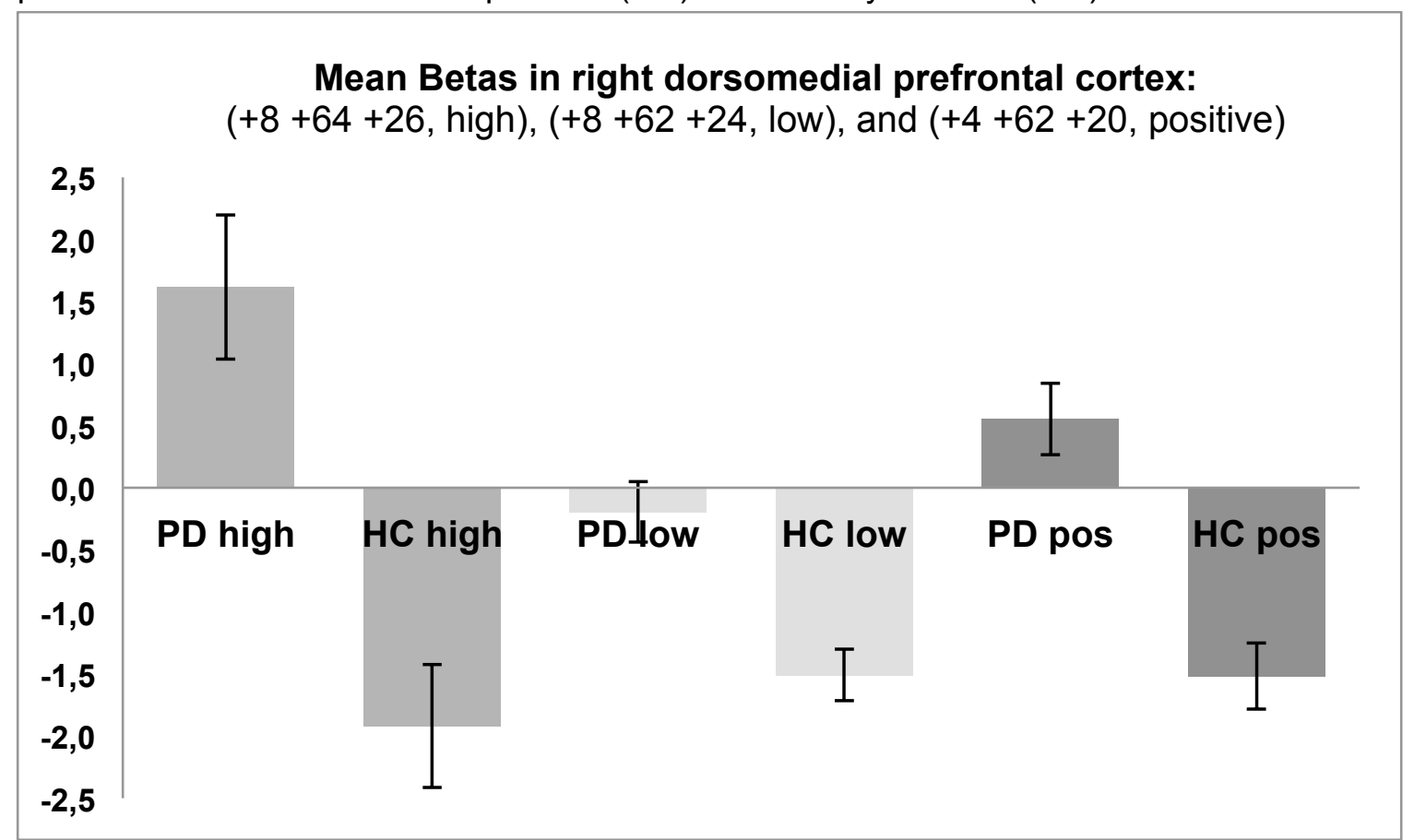

MNI coordinates refer to the location of the maxima. Results are considered significant at $P_{\mathrm{FWE}}<0.05$ small volume correction. 



\section{CHAPTER 4}

Cognitive disorders in PARKINSON'S disease: ConfiRmation of A SPECTRUM OF SEVERITY

Parkinsonism and Related Disorders, 21: 1299-1305, 2015

Kathy Dujardin, Anja J.H. Moonen, Hélène Behal, Luc Defebvre, Alain Duhamel, Annelien A. Duits, Lucie Plomhause, Celine Tard, Albert F.G. Leentjens 


\section{ABstract}

Introduction: Clinical presentation and progression of cognitive disorders in Parkinson's disease (PD) is heterogeneous. Our objective was to confirm prospectively a previous exploratory cluster analysis based on retrospective data that identified five cognitive phenotypes in PD.

Methods: A model-based confirmatory cluster analysis was conducted on the results of neuropsychological tests administered in 156 PD patients from two European movement disorder centers (Lille, $n=81$; Maastricht, $n=75$ ). The number of clusters was determined on the basis of statistical criteria as well as clinical plausibility. A factorial discriminant analysis assessed the quality of the clusters' separation.

Results: A five-cluster model was statistically superior and clinically the most relevant. These clusters can be described as follows: 1) cognitively intact patients with high levels of performance in all cognitive domains $(25.64 \%), 2)$ cognitively intact patients who were slightly slower than those in cluster $1(26.92 \%), 3)$ patients with deficits in executive functions $(37.18 \%), 4)$ patients with severe deficits in all cognitive domains, particularly executive functions $(3.20 \%), 5)$ patients with severe deficits in all cognitive domains, particularly working memory and recall in verbal episodic memory (7.05\%). The groups differed in terms of age, apathy and frequency of hallucinations that were all higher in the clusters with cognitive deficits, and the duration of formal education was lower in those groups.

Conclusion: We confirm our previous exploratory analysis. Cognitive disorders in PD patients are heterogeneous and can be separated in five clusters ranging from patients with performance in the normal range to patients with severe disorders in all cognitive domains. 


\section{INTRODUCTION}

Cognitive deficits are common in Parkinson's disease (PD), even in non-demented patients. The prevalence of mild cognitive impairment in PD patients ranges from 20 to $50 \%{ }^{1}$ and those patients have a higher risk of developing dementia. However, some patients have no cognitive impairment at all and their prevalence ranges from 40 to $75 \%{ }^{2,3}$ There is thus a substantial heterogeneity in the clinical presentation and progression of cognitive disorders in PD. Only few studies have used a data-driven approach, such as cluster analysis, to identify the phenotypic profiles that arise from performance at a broad range of tests without a priori assumptions (for a review, see Ref 4 ). In a previous study, ${ }^{4}$ we used an exploratory cluster analysis based on retrospective data in a large group $(n=557)$ of $P D$ patients who underwent a standardized neuropsychological assessment. We identified five well-separated clusters, ranging from cognitively intact patients with rather high levels of performance in each cognitive domain to very severely impaired patients, with a progressive severity gradient. Recall in episodic verbal memory, speed of processing and executive functions contributed the most in separating the clusters. However, our study had several limitations. Namely, as data collection was based on standardized clinical practice, some cognitive domains were assessed very briefly or not at all (e.g., language). Hence, the obtained classification needed validation in an independent sample with data collected in a prospective way. Such validation is an essential prerequisite for the investigation of anatomical and functional correlates of these clusters.

In the present study, an extensive neuropsychological assessment was performed in a different group of PD patients with the aim of confirming the previously found five cognitive phenotypes. If our previous results are replicated, this classification will provide a solid support for investigation of the anatomical and functional correlates of each of the identified profiles.

\section{METHODS}

\section{Participants}

One hundred fifty-six PD patients participated in the study. All patients met the United Kingdom Brain Bank criteria for idiopathic $P D^{5}$ and none was suffering from a neurological disease other than PD. To avoid missing data, patients with moderate and severe dementia (defined as a score $>1$ at the Clinical Dementia Rating ${ }^{6}$ and 
according to the Movement Disorders criteria ${ }^{7}$ ) and those older than 80 years were excluded. In order to achieve a sample as representative as possible of the entire PD population, there were no additional exclusion criteria.

Patients were recruited among the outpatients of two independent European movement disorder centers, in Lille, France $(n=81)$ and Maastricht, the Netherlands $(n=75)$. Data were collected from March 2013 to August 2014. All participants gave their informed consent to participation in the study, which had been approved by the local institutional review boards (CPP Nord-Ouest IV, 2012-A 01317-36, ClinicalTrials.gov Identifier: NCT01792843).

\section{Neuropsychological assessment}

Subjects underwent an extensive neuropsychological assessment including the Mini Mental State Examination (MMSE) and the Mattis dementia rating scale (MDRS) ${ }^{8}$ for global cognition and standardized tests representing five cognitive domains: 1) attention and working memory (Digit span forward and backward, ${ }^{9}$ Symbol Digit Modalities Test $(\mathrm{SDMT})^{10}$ 2) executive functions (Trail Making Test B/A ratio ${ }^{11}$, the interference index and the number of errors in the interference condition of a 50-item version of the Stroop word color test and a 1-minute phonemic word generation task performed in single and alternating conditions) 3) verbal episodic memory (Hopkins verbal learning test ${ }^{12}$ ), 4) language (the 15-item short form of the Boston naming test $^{13}$ and animal names generation task in 1 minute), 5) visuospatial functions (the short version of the judgment of line orientation test ${ }^{14}$ ). Scores at these neuropsychological tests constitute the cluster variables, which were used to build the clusters.

\section{Clinical and demographical variables}

Detailed demographic and disease related variables were recorded likewise. All the patients' medications were checked and doses of antiparkinsonian medication were converted to levodopa equivalent daily dose (LEDD) according to the algorithm by Tomlinson et al. ${ }^{15}$ The Movement Disorders Society - Unified Parkinson Disease Rating Scale (MDS-UPDRS) ${ }^{16}$ was administered to assess non-motor and motor experiences of daily living (part I and II), severity of motor symptoms (part III), complications of therapy (part IV), and disease stage (Hoehn \& Yahr score). The severity of depression, apathy and anxiety symptoms was quantified with the 17-item Hamilton Depression Rating Scale (HAMD) $)^{17}$, the Lille Apathy Rating Scale (LARS) $)^{18}$ 
and the Parkinson Anxiety Scale (PAS) ${ }^{19}$, respectively. Instrumental activities of daily living (IADL) were scored with the Lawton IADL scale. ${ }^{20}$

We also checked for the presence (yes/no) of high blood pressure (defined as systolic/diastolic numbers higher than $140 / 90 \mathrm{~mm} \mathrm{Hg}$ ), hypercholesterolemia, diabetes, history of myocardial infarction, of lower limb arteriopathy, and cerebrovascular disease. The presence of sleep apnea and of clinical signs of REM sleep behavior disorders (standard patient interview) was also recorded. All patients were assessed after having received their usual antiparkinsonian medication and were in their "best on" state.

\section{Power calculation}

As no standardized method exists to calculate the number of subjects required to perform cluster analysis, the number of subjects was defined according to the number of the clusters identified in our previous study and the proportion of subjects in each cluster. ${ }^{4}$ As the smallest cluster included $13 \%$ of subjects, we decided to enroll at least 150 subjects in order to reach a minimum of 20 subjects in each category.

\section{Statistical Analysis}

The numerical variables were described as means and standard deviations and the categorical variables as frequencies and percentages.

\section{Cluster analysis}

The 19 variables derived from the neuropsychological tests were considered for the cluster analysis (CA). The CA was based on the k-means method in which similarity between individuals is measured using the usual Euclidian distance. We performed several analyses with different numbers of clusters and the optimal number was determined by consensus among three statistical properties: local peaks of the cubic cluster criterion (CCC) and pseudo F combined to a bend in the $\mathrm{R}^{2}$ (ratio between the inter-cluster variation and the total variation) increase. The number of subjects in each cluster and face validity as judged by experts (K.D., A.L., A.M., A.A.D) were also considered. All the patients were included in the CA. 


\section{Characterization of clusters}

To compare the clusters on quantitative variables, an analysis of variance with generalized linear model with Tukey correction for multiple testing was performed when data distribution was normal and a Kruskal-Wallis test was used for non-normal distributions. Pairwise comparisons were applied. Categorical variables were compared with Chi-square test or a Fisher's exact test when the expected cell frequency was $<5$. A p-value $<0.05$ was considered significant. Cluster comparisons were performed for the cognitive variables (the cluster variables) as well as for the clinical and demographical variables (the descriptive variables).

To evaluate the quality of clusters separation, a first factorial discriminant analysis was performed with the clusters as dependent and the cognitive features as independent variables. The correlations between these variables and the discriminant scores were computed to identify the variables contributing most to the separation of clusters. A second factorial discriminant analysis was performed on the clinical and demographical variables to illustrate the clusters profile.

\section{RESULTS}

A five-cluster model was both statistically superior (see Table 1) and clinically the most relevant. The characteristics of the identified clusters and results of group comparisons are shown in Table 2.

Table 1. K-means clustering: testing subsequent clustering analysis models.

\begin{tabular}{|c|c|c|c|}
\hline Number of classes & Cubic Custer Criterion & Pseudo F & $\mathbf{R}^{2}$ \\
\hline 2 & 5.53 & 112.59 & 0.42 \\
\hline 3 & 7.14 & 78.97 & 0.51 \\
\hline 4 & 7.31 & 61.95 & 0.55 \\
\hline 5 & 10.53 & 60.46 & 0.62 \\
\hline 6 & 12.02 & 54.96 & 0.65 \\
\hline 7 & 11.63 & 49.71 & 0.67 \\
\hline 8 & 10.20 & 43.75 & 0.67 \\
\hline
\end{tabular}

Clusters 1 and 2 were formed by cognitively intact patients. Patients in cluster 1 $(n=40)$ had high levels of performance in all the assessed cognitive domains. Patients in cluster $2(n=42)$ had no deficits but performed slightly slower and named fewer animals than those in cluster 1. Patients in cluster $3(n=58)$ had a lower score at the MDRS than patients in the two first clusters but had preserved overall cognitive 


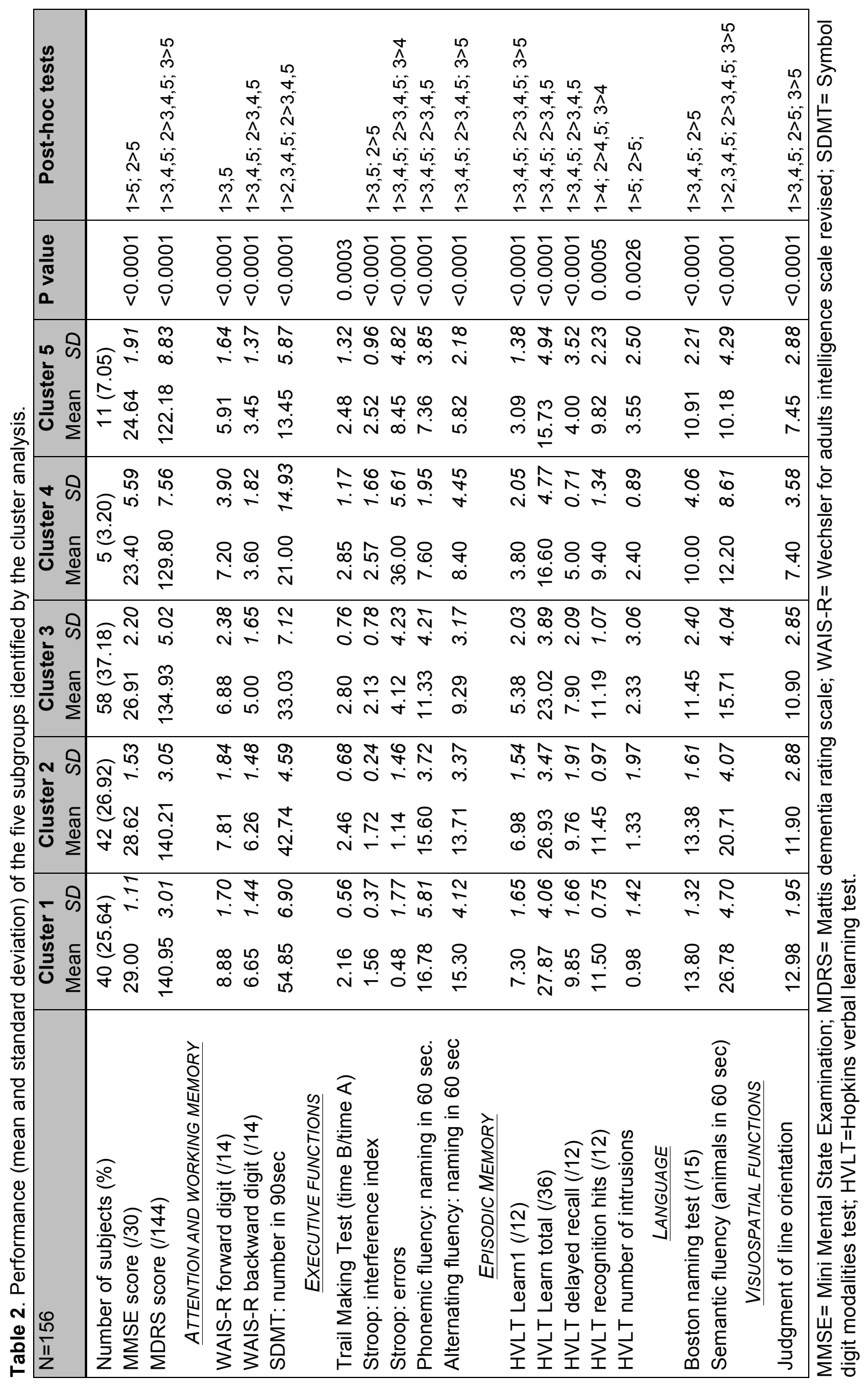


efficiency. They showed lower scores than the patients in clusters 1 and 2 in attention, working memory and executive functions. In episodic verbal memory, they recalled less items but recognition was in the normal range. For language and visuospatial functions, they performed lower than patients in cluster 1. Patients in cluster $4(n=5)$ and cluster $5(n=11)$ had impaired overall efficiency and severe deficits in all cognitive domains. Pairwise comparisons with cluster 4 were open to criticism due to the small number of subjects in that group but were provided for information purposes. Although we observed no significant difference between cluster 4 and 5 , patients in cluster 4 seemed to have more severe deficits in executive functions. Conversely, those in cluster 5 performed slower and had lower scores in tests assessing working memory and recall in verbal episodic memory. The descriptive variables for the different clusters are shown in Table 3.

No demographic or clinical parameters differentiated clusters 1 and 2. Patients in cluster 1 were significantly younger and received more years of formal education than those in clusters 3, 4 and 5. The five clusters did not significantly differ regarding disease duration, severity of motor symptoms, frequency of axial signs, frequency of dyskinesia, antiparkinsonian medication, frequency of REM sleep behavior disorders, frequency of vascular risk factors, and severity of depressive symptoms. Dementia and hallucinations were more frequent in cluster 4 and 5 than in the others. Regarding anxiety symptoms, we only found a difference between cluster 1 and 3 , with more avoidance symptoms in cluster 3 .

The results of the discriminant analysis performed on the neuropsychological data are shown in Figure 1. The separation of the clusters on the two first discriminant scores $\left(S_{1}\right.$ and $\left.S_{2}\right)$ was mainly explained by the number of correct responses at the SDMT (correlation with $S_{1}: r_{\mathrm{S} 1}=0.84$, correlation with $S_{2}: r_{\mathrm{S} 2}=0.46$ ), the number of errors at the Stroop test $\left(r_{\mathrm{S} 1}=-0.85, r_{\mathrm{S} 2}=0.49\right)$ and animal fluency $\left(r_{\mathrm{S} 1}=0.71, r_{\mathrm{S} 2}=0.43\right)$. On the basis of these cognitive variables, $87 \%$ of patients were correctly classified. The discriminant analysis performed on the demographic and clinical variables revealed that dementia $\left(r_{\mathrm{S} 1}=0.66\right)$, IADL $\left(r_{\mathrm{S} 1}=-0.61\right)$, score on the LARS $\left(r_{\mathrm{S} 1}=0.43\right)$, age $\left(r_{\mathrm{S} 1}=0.41\right)$, and education duration $\left(r_{\mathrm{S} 1}=-0.46\right)$ best discriminated the clusters according to the first discriminant score. The discriminant power of the second score was not significant. 


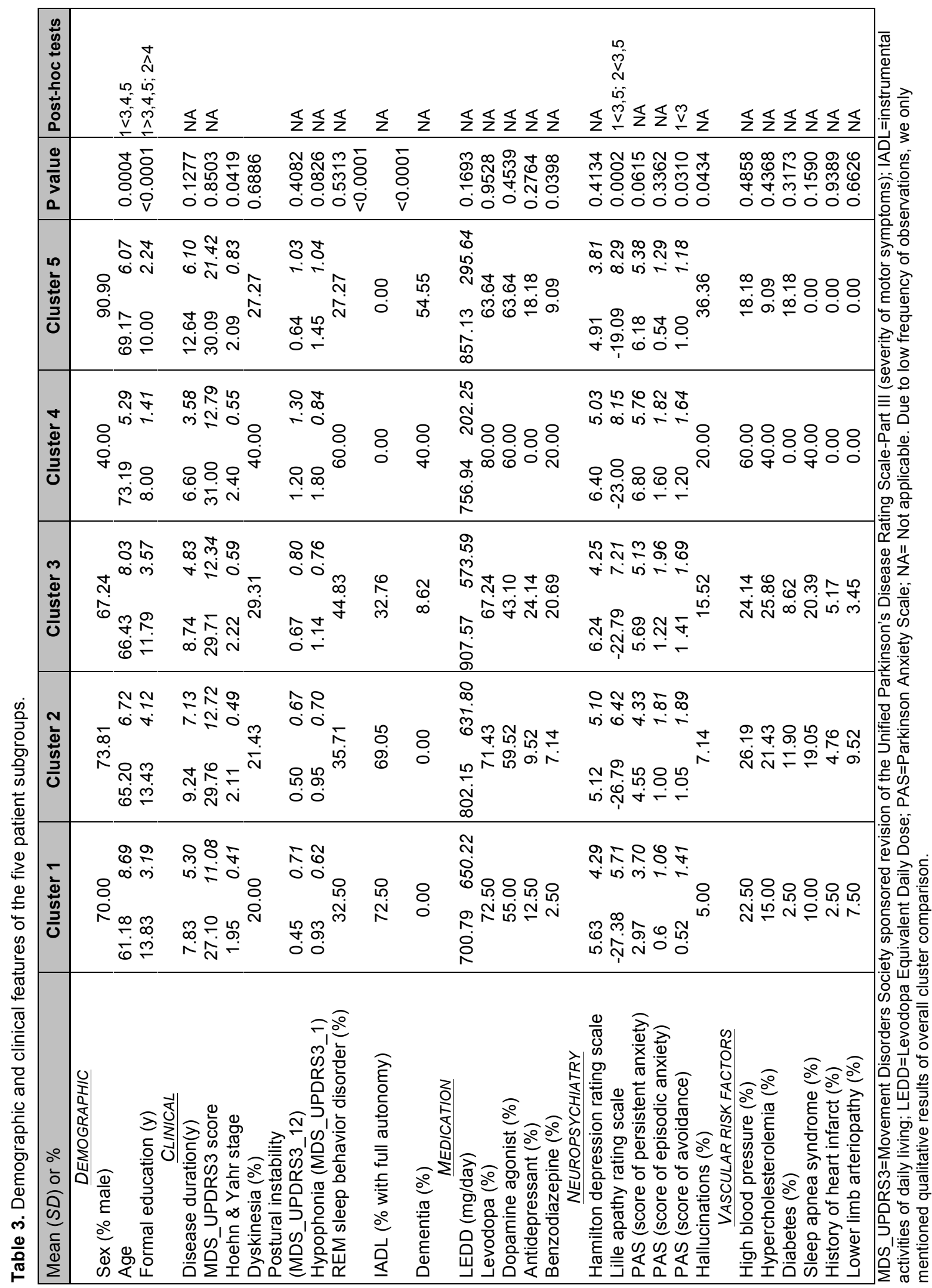




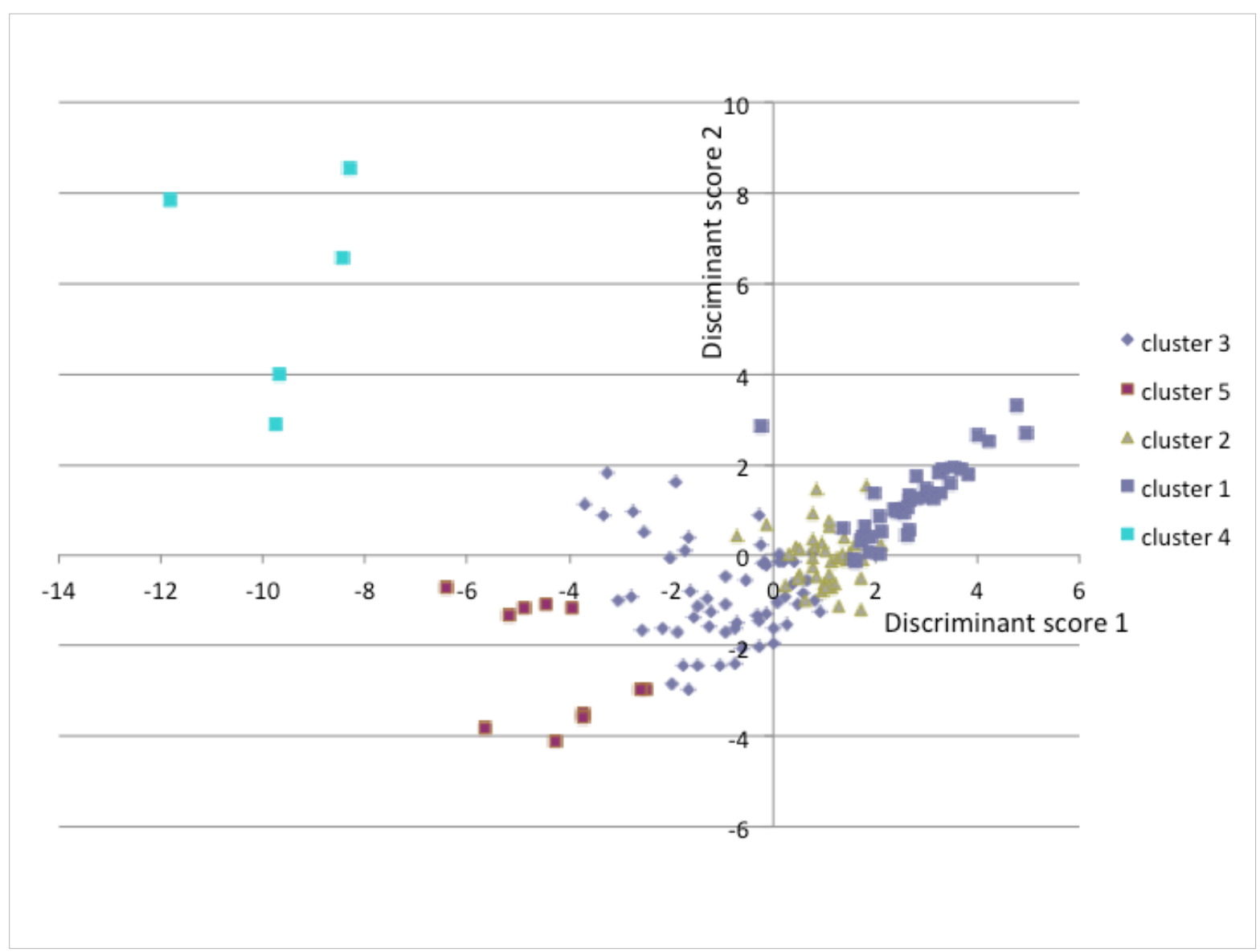

Figure 1. Graphic representation of the cluster separation by the factorial discriminant analysis on the cognitive variables (scatter plot for the two first discriminant scores: $S_{1}$ and $\left.\mathrm{S}_{2}\right)$.

\section{Discussion}

Our data-driven approach in a group of 156 patients with PD confirmed that presentation of cognitive disorders in PD is heterogeneous and can be separated into five different phenotypes with a spectrum going from patients with high levels of performance in the main cognitive domains to patients with severe deficits in all cognitive domains.

Compared to our previous study, the proportion of patients in each group is slightly different. There are less severely cognitively impaired patients (10\% vs. $25 \%$ ) and more patients with mild cognitive symptoms (37\% vs. $13 \%)$. These differences probably reflect differences in the inclusion criteria: in the first study, we considered all patients with PD irrespective of their cognitive status but in the present study, we did not enroll patients with moderate and severe dementia in order to avoid incomplete data or threshold effects at the neuropsychological tests. The identified clusters though are an exact replication of our previous results. Moreover, the 
discriminant analysis on the cognitive variables showed that although we did not use exactly the same tests as before, the most discriminant variables correspond to exactly the same cognitive domains: speed of processing, inhibition and semantic fluency. With these discriminant variables and the mahalanobis distance, $83 \%$ of the patients were well classified. The identified cognitive profiles thus seem to be very stable. Considering this stability, predictive scores could be built for classifying the patients in the different clusters on the basis of their cognitive characteristics. It also provides a solid support for investigation of the anatomical and functional markers of each of the identified cognitive profiles.

Regarding the cognitive variables, the only differences between the two clusters of cognitively intact patients concerned speed of processing and semantic fluency. The profile of patients in cluster 3 is close to that described in PD with dysexecutive mild cognitive impairment $(\mathrm{MCl})$ with low performance at tests assessing attention and executive functions but performance in the normal range in the other cognitive domains. ${ }^{21}$ In both last clusters, eight patients (two in cluster 4 and six in cluster 5) met the criteria for mild PD dementia after clinical and neuropsychological assessment and the others had a profile close to that described in multiple domains $\mathrm{MCl}$ with low performance in several cognitive domains. Although patients in these two groups have global cognitive decline, those in cluster 5 have predominant memory deficits, which may reflect more comorbid Alzheimer disease pathology. A more systematic search for cerebrospinal fluid markers and genetic characterization could also interestingly contribute to further define cognitive phenotypes. Although the low number of subjects prevented post-hoc comparisons of clusters 4 and 5 , one could hypothesize that cluster 4 is characterized by faster disease progression and cognitive decline than cluster 5 (shorter disease duration for similar severity of motor and cognitive symptoms). Older age at onset and shorter education duration also seem to play a role in this faster progression of cognitive decline. The specificity of this group is supported by the results of the factorial discriminant analysis.

According to the dual syndrome hypothesis ${ }^{22}$ we found patients with deficits reflecting fronto-striatal dysfunction (cluster 3 ) but did not find a cluster of patients exhibiting only deficits indicative of posterior cortical and temporal lobe dysfunction. When such deficits were observed, they seem to be in addition to fronto-striatal dysfunction as in clusters 4 and 5 . However, this would require verification by classifying each patient on the characteristics of the dual syndrome. 
Regarding the demographic and clinical variables, the groups did not differ in terms of disease duration, severity of the motor symptoms, and frequency of dyskinesia, frequency of axial signs or medication. There was also no difference in the frequency of the vascular risk factors. The main differences concerned age, apathy and frequency of hallucinations that were higher in the clusters with cognitive deficits as well as duration of formal education that was lower in those groups. This agrees with previous studies showing that age, ${ }^{23-25}$ years of formal education, ${ }^{25,26}$ apathy ${ }^{27,28}$ and hallucinations ${ }^{23,25,28}$ are associated with a higher risk of cognitive decline in patients with PD. However, compared with our previous study ${ }^{4}$ and several other studies, ${ }^{3,23}$ cognitive impairment was not associated with longer disease duration, more severe motor symptoms or postural instability. However, this association was not observed systematically $^{29}$ and may vary depending on the proportion of patients with dementia. ${ }^{25}$

Several limitations of the current study should be considered. Firstly, the number of subjects in clusters 4 and 5 was low and prevented pairwise group comparisons, although descriptive statistics suggest differences on several cognitive and clinical parameters. Moreover, the discriminant analysis (see Figure 1) clearly suggests differences between these two clusters that were difficult to identify in absence of pairwise comparisons. Secondly, patients were recruited from tertiary referral centers and therefore, they cannot be considered as fully representative of the general population. However, recruiting from movement disorders centers has the advantage to provide reliable records containing PD specific information relevant for inclusion/exclusion criteria. Moreover, replication of our previous results is a strength of the present study and is an important support for research of specific anatomical and functional markers of the identified cognitive profiles. Thirdly, center differences were observed on some neuropsychological variables despite similar inclusion criteria in the both centers and we cannot exclude a center effect on the identified clusters. However, we repeated the analyses considering only the data from the center of Lille and also found five clusters with similar cognitive characteristics and frequencies. Fourthly, we used a cross-sectional design to describe the cognitive profiles and a follow-up of the identified groups is now essential to determine whether the identified profiles differ in terms of disease progression and prognosis. Nevertheless, even though no conclusion on the course of cognitive decline can be drawn from this cross-sectional study, replication of our 
results justifies the view that slowed mental speed could be considered as an early marker of cognitive decline in PD.

In summary, through a data-driven approach on an independent sample, we have replicated the identification of five well-separated cognitive phenotypes in PD. The stability of the identified profiles prompts to investigate their anatomical and functional correlates in order to identify specific markers of each group. 


\section{REFERENCES}

1. I. Litvan, D. Aarsland, C.H. Adler, J.G. Goldman, J. Kulisevsky, B. Mollenhauer, et al., MDS Task Force on mild cognitive impairment in Parkinson's disease: critical review of PD-MCl, Mov. Disord. 26 (2011) 1814-1824.

2. D. Muslimovic, B. Post, J.D. Speelman, B. Schmand, Cognitive profile of patients with newly diagnosed Parkinson disease, Neurology. 65 (2005) 1239-1245.

3. C. Janvin, D. Aarsland, J.P. Larsen, K. Hugdahl, Neuropsychological profile of patients with Parkinson's disease without dementia, Dement Geriatr Cogn Disord. 15 (2003) 126-131.

4. K. Dujardin, A.F.G. Leentjens, C. Langlois, A.J.H. Moonen, A.A. Duits, A.-S. Carette, A. Duhamel. The spectrum of cognitive disorders in Parkinson's disease: a data-driven approach, Mov. Disord. 28 (2013) 183-189.

5. W.R. Gibb, A.J. Lees, The relevance of the Lewy body to the pathogenesis of idiopathic Parkinson's disease, J. Neurol. Neurosurg. Psychiatr. 51 (1988) 745-752.

6. J.C. Morris, The Clinical Dementia Rating (CDR): current version and scoring rules, Neurology. 43 (1993) 2412-2414.

7. M. Emre, D. Aarsland, R. Brown, D.J. Burn, C. Duyckaerts, Y. Mizuno, et al., Clinical diagnostic criteria for dementia associated with Parkinson's disease, Mov. Disord. 22 (2007) 1689-707-quiz 1837.

8. S. Mattis, Mental status examination for organic mental syndrome in the elderly patient, in: L. Bellak, K. T (Eds.), Geriatric Psychiatry, Grune and Stratton, New York, 1976: pp. 77-121.

9. D. Wechsler, WAIS-R: Wechsler adult intelligence scale, revised, 1986.

10. A. Smith, Symbol Digits Modalities Test, Western Psychological Services, Los Angeles, 1982.

11. R.M. Reitan, TMT, Trail Making Test A \& B, South Tucson, 1992.

12. J. Brandt, R. Benedict, Hopkins Verbal Learning Test-Revised. Lutz, FL: Psychological Assessment Resources, 2001.

13. R.E. Graves, S.C. Bezeau, J. Fogarty, R. Blair, Boston naming test short forms: a comparison of previous forms with new item response theory based forms, J Clin Exp Neuropsychol. 26 (2004) 891-902.

14. A.L. Benton, N.R. Varney, K.D. Hamsher, Visuospatial judgment. A clinical test, Arch. Neurol. 35 (1978) 364-367.

15. C.L. Tomlinson, R. Stowe, S. Patel, C. Rick, R. Gray, C.E. Clarke, Systematic review of levodopa dose equivalency reporting in Parkinson's disease, Mov. Disord. 25 (2010) 2649-2653.

16. C.G. Goetz, B.C. Tilley, S.R. Shaftman, G.T. Stebbins, S. Fahn, P. Martinez-Martin, et al., Movement Disorder Society-sponsored revision of the Unified Parkinson's Disease Rating Scale (MDS-UPDRS): scale presentation and clinimetric testing results, Mov. Disord. 23 (2008) 2129-2170.

17. M. Hamilton, A rating scale for depression, J. Neurol. Neurosurg. Psychiatr. 23 (1960) 56-62.

18. P. Sockeel, K. Dujardin, D. Devos, C. Deneve, A. Destée, L. Defebvre, The Lille apathy rating scale (LARS), a new instrument for detecting and quantifying apathy: validation in Parkinson's disease, J. Neurol. Neurosurg. Psychiatr. 77 (2006) 579-584.

19. A.F.G. Leentjens, K. Dujardin, G.M. Pontone, S.E. Starkstein, D. Weintraub, P. 
Martinez-Martin, The Parkinson Anxiety Scale (PAS): development and validation of a new anxiety scale, Mov. Disord. 29 (2014) 1035-1043.

20. M.P. Lawton, E.M. Brody, Assessment of older people: self-maintaining and instrumental activities of daily living, Gerontologist. 9 (1969) 179-186.

21. I. Litvan, J.G. Goldman, A.I. Tröster, B.A. Schmand, D. Weintraub, R.C. Petersen, et al., Diagnostic criteria for mild cognitive impairment in Parkinson's disease: Movement Disorder Society Task Force guidelines, Mov. Disord. 27 (2012) 349-356.

22. A.A. Kehagia, R.A. Barker, T.W. Robbins, Cognitive Impairment in Parkinson's Disease: The Dual Syndrome Hypothesis, Neurodegener Dis. 11 (2013) 79-92.

23. E.Y. Uc, M.P. McDermott, K.S. Marder, S.W. Anderson, I. Litvan, P.G. Como, et al., Incidence of and risk factors for cognitive impairment in an early Parkinson disease clinical trial cohort, Neurology. 73 (2009) 1469-1477.

24. J.B.M. Anang, J.-F. Gagnon, J.-A. Bertrand, S.R. Romenets, V. Latreille, M. Panisset, et al., Predictors of dementia in Parkinson disease: a prospective cohort study, Neurology. 83 (2014) 1253-1260.

25. K. Zhu, J.J. van Hilten, J. Marinus, Predictors of dementia in Parkinson's disease; findings from a 5-year prospective study using the SCOPA-COG, Parkinsonism Relat. Disord. 20 (2014) 980-985.

26. M.T.M. Hu, K. Szewczyk-Królikowski, P. Tomlinson, K. Nithi, M. Rolinski, C. Murray, et al., Predictors of cognitive impairment in an early stage Parkinson's disease cohort, Mov. Disord. 29 (2014) 351-359.

27. K. Dujardin, P. Sockeel, M. Delliaux, A. Destée, L. Defebvre, Apathy may herald cognitive decline and dementia in Parkinson's disease, Mov. Disord. 24 (2009) 23912397.

28. D. Aarsland, J.-P. Taylor, D. Weintraub, Psychiatric issues in cognitive impairment, Mov. Disord. 29 (2014) 651-662.

29. A. McKinlay, R.C. Grace, J.C. Dalrymple-Alford, D. Roger, Cognitive characteristics associated with mild cognitive impairment in Parkinson's disease, Dement Geriatr Cogn Disord. 28 (2009) 121-129. 



\section{CHAPTER 5}

REDUCED GREY MATTER DENSITY ASSOCIATED WITH EARLY COGNITIVE DECLINE IN PARKINSON'S DISEASE: A VOXEL-BASED MORPHOMETRY STUDY

Anja J.H. Moonen, Albert F.G. Leentjens, Annelien A. Duits, Luc Defebvre, Christine

Delmaire, Renaud Lopes, Paul A. Hofman, Frank C. van Bussel, Kathy Dujardin 


\section{ABSTRACT}

Introduction: Cognitive symptoms occur already in early stages of Parkinson's disease (PD). Here we aimed to identify patterns of grey matter (GM) atrophy in PD patients with different cognitive profiles.

Methods: Patient subgroups (total $\mathrm{N}=124$ ) were previously defined by a data-driven approach, resulting in four cognitive profiles ranging from cognitively intact patients, to patients with mental slowing but otherwise cognitively intact, to a dysexecutive syndrome, to patients with severe cognitive dysfunction in all cognitive domains. We used optimized voxel-based morphometry (VBM-Dartel) to analyze whole brain structural T1-weighted MRI scans and compared GM volume in all groups compared to the cognitively intact group.

Results: Patients with slight mental slowing, but overall intact cognition, showed reduced GM density in temporal, parietal and frontal regions, whereas patients with a profile close to dysexecutive mild cognitive impairment showed increased GM atrophy in the parietal-temporal lobe, posterior cingulate, bilateral hippocampus and bilateral amygdala. Patients with severe cognitive deficits in multiple domains showed a similar pattern of GM atrophy with additional GM loss in the left medial temporal lobe and left parahippocampal gyrus. These results were robust for differences in center, gender, and level of education. Only patients with severe cognitive deficits showed differences in GM atrophy independent of the effect of age.

Conclusion: Our results revealed neuroanatomical markers of early cognitive decline in PD patients with reduced mental speed but otherwise intact cognition. Age at evaluation appeared to be an important predictor of differences in GM loss. However, in patients with severe cognitive deficits we found GM abnormalities unrelated to age that may resemble more widespread Lewy body pathology with potential comorbid Alzheimer's disease pathology. 


\section{INTRODUCTION}

Cognitive impairment in Parkinson's disease (PD) already occurs at early stages of the disease. Up to $20 \%$ of PD patients report mild cognitive impairment at time of diagnosis and approximately $30 \%$ of PD patients convert to dementia during the course of the disease. ${ }^{1}$ Yet, a substantial part of PD patients are cognitively intact, even years after being diagnosed. ${ }^{2-4}$ Recent studies support the large heterogeneity in the range of cognitive deficits in PD, and several have attempted to identify distinctive cognitive profiles. ${ }^{5}$ However, many studies investigated cognitive dysfunction using previously defined categories such as PD patients without cognitive impairment (PD-NC), with mild cognitive impairment (PD-MCl), and PD patients with dementia (PDD). In a previous study, ${ }^{6}$ we identified five cognitive clusters through an exploratory cluster analysis using retrospective neuropsychological test data from a large sample $(n=557)$ of PD patients. More recently, we replicated and confirmed the identification of the same five cognitive phenotypes by performing a model-based confirmatory cluster analysis on prospective neuropsychological data from an independent sample of PD patients $(n=156){ }^{7}$ The five clusters ranged from cognitively intact patients, to patients with mental slowing but otherwise cognitively intact, to a dysexecutive syndrome, to patients with severe cognitive dysfunction in all cognitive domains.

In the present study, we aimed to identify the neuroanatomical correlates of each of the cognitive phenotypes using structural magnetic resonance imaging (MRI). More specifically we were looking for regional differences in the rate of grey matter (GM) atrophy between these groups. We hypothesized that all groups would show increased loss of GM compared to the cognitively intact group, with a progressive severity gradient. An additional hypothesis concerned specific GM atrophy reflecting comorbid Alzheimer's disease (AD) pathology (i.e., hippocampal or medial temporal lobe atrophy) in those patients groups with more profound memory deficits. We followed an explorative whole brain approach, with post hoc region-ofinterest analyses. Investigating neuroanatomical correlates of cognitive profiles may identify markers of early cognitive decline in PD. 


\section{Methods}

Participants

One hundred fifty-six patients with idiopathic Parkinson's disease participated in the study. ${ }^{7}$ Patients were recruited from two independent European movement disorders clinics in Maastricht, the Netherlands $(n=75)$ and Lille, France $(n=81)$. The diagnosis of idiopathic PD was established by means of the United Kingdom Brain Bank diagnostic criteria for PD. ${ }^{8}$ Patients with moderate and severe dementia (defined as a score $>1$ at the Clinical Dementia Rating ${ }^{9}$ and following the Movement Disorders criteria, ${ }^{10}$ patients older than 80 years and patients with neurodegenerative disorders other than PD were excluded from the study. Patients treated with deep brain stimulation or those meeting contra-indications for MRI were excluded likewise. All patients were using stable doses of antiparkinsonian medication and were tested in their "on" state. All patients gave informed consent prior to participation, which has been approved by the local institutional review boards (ClinicalTrials.gov Identifier: NCT01792843, CPP Nord-Ouest IV, 2012-A 01317-36).

\section{Clinical assessment}

All patients underwent a thorough neuropsychiatric examination ${ }^{7}$ consisting of the Movement Disorder Society - Unified Parkinson's disease Rating Scale ${ }^{11}$ (MDSUPDRS, sections I-IV) to measure severity and experiences of non-motor and motor symptoms, the Hoehn and Yahr staging scale to measure disease stage (Hoehn \& Yahr score), the 17-item Hamilton Depression Rating Scale ${ }^{12}$ (HAMD) to assess depressive symptoms, the Parkinson Anxiety Scale ${ }^{13}$ (PAS) to measure anxiety symptoms, the Lille Apathy Rating Scale ${ }^{14}$ (LARS) to measure levels of apathy, and the Lawton Instrumental Activities of Daily Living ${ }^{15}$ (IADL) to score activities of daily living. In addition, we checked for the presence of several medical conditions such as high blood pressure, hypercholesterolemia, diabetes, history of myocardial infarction, history of lower limb arteriopathy, cerebrovascular disease, sleep apnea, and symptoms of Rapid Eye Movement (REM) sleep behavior disorders.

\section{Neuropsychological assessment}

In addition to the neuropsychiatric examination, all patients underwent an extensive neuropsychological test battery ${ }^{7}$ measuring global cognition (Mini Mental State Examination, ${ }^{16}$ MMSE; Mattis dementia rating scale, ${ }^{17}$ MDRS) as well as multiple cognitive domains. Attention and working memory was assessed with the Digit span forward and backward ${ }^{18}$ and the Symbol Digit Modalities Test ${ }^{19}$ (SDMT). Executive 
functioning was measured with the Trial Making Test ${ }^{20}$ (TMT, B/A ratio), the interference index of the Stroop word color test, and the word generation task (i.e., single and alternating phonemic conditions). Verbal episodic memory was measured by means of the Hopkins Verbal Learning Test $^{21}$ (HVLT). We used the Boston Naming test ${ }^{22}$ and a categorical word generation task (i.e., animals) to assess language, and the short version of the Benton Judgment Line Orientation test ${ }^{23}$ (BJLO) to measure visuospatial functioning.

\section{Cognitive clusters}

Model-based confirmatory cluster analysis based on the neuropsychological test scores from all 156 patients revealed the following five cognitive clusters (see ref $\left.{ }^{7}\right]$ for a detailed description of the statistical procedure): 1) Cognitively intact patients with above average performance in all cognitive domains (25.64\%), 2) Cognitively intact patients with slight mental slowing (26.92\%), 3) Patients showing mild cognitive deficits in attention, working memory, executive functioning, language and visuospatial functioning and slight deficits in episodic verbal memory with intact recognition $(37.18 \%)$, 4) Patients with impaired overall cognitive efficiency and severe deficits in all cognitive domains, particularly executive functioning (3.2\%), and 5) Patients with impaired overall cognitive efficiency and severe deficits in all cognitive domains, particularly working memory and recall in verbal episodic memory (7.05\%). Out of the whole sample of 156 patients, 133 patients have had an MRI scan. An extensive data quality check resulted in the exclusion of 9 patients ( 7 due to large motion artifacts and 2 due to lesions). Also, due to the small number of subjects in cluster 4 and 5 , these were collapsed and considered as one cluster for further imaging analyses. Due to these adjustments, the remaining four groups were not completely identical to the original clusters, and we will use the terms 'patient group' or 'cognitive profile' rather than 'clusters' from now on.

\section{MRI Imaging Data Acquisition and Analysis}

Magnetic resonance images were acquired with a 3T whole-body system (Philips Achieva, Philips Medical Systems, Best, the Netherlands) with an eight-channel SENSE head coil. High-resolution 3-dimensional T1-weighted images were acquired in the sagittal plane (TR 7200 ms. TE 3300 ms, TI 900 ms; 256 × 256 matrix, FOV $256 \times 240 \mathrm{~mm}^{2}, 1 \mathrm{~mm}$ isotropic voxel), yielding 176 contiguous slices through the head. 


\section{Optimized Voxel-Based Morphometry}

Images were processed using the voxel-based morphometry (VBM) toolbox version 8 (VBM8; http://dbm.neuro.uni-jena.de/vbm/) within SPM8 (Welcome Trust Centre for Neuroimaging, London, UK; http://fil.ion.ucl.ac.uk/spm/software/spm8/) in MATLAB 7.14 (Math-works Inc., Natick, MA, USA). Default settings were used unless otherwise specified. Prior to image analyses, all images were screened for artifacts and large anatomical abnormalities and manually re-orientated in SPM8, such that they were centered on the anterior commissure. For spatial normalization we applied the high-dimensional Dartel normalization approach (VBM-Dartel). ${ }^{24}$ In order to obtain regional grey matter values and account for individual differences in global brain size, the next step involved modulated normalization followed by segmentation of images into GM, white matter (WM) and cerebrospinal fluid (CSF). After checking the homogeneity of the sample, the normalized modulated images were smoothed with a standard Gaussian kernel of $8 \mathrm{~mm}$, full width at half maximum.

\section{Statistical analysis}

The smoothed, modulated, normalized grey matter data were analyzed in SPM8. Ttests were conducted in order to identify differences in whole brain gray matter volume between the patient groups. An absolute threshold of 0.1 was used, by which only voxels with $\mathrm{GM}$ values $>0.1$ were counted. Correction for global volume change was not necessary, since we already applied this correction directly to the data as part of the (non-linear) modulation step (recommended by the VBM8 manual). We ran the analysis twice, once with center, gender and education entered as covariates into the design matrix and once including age as an additional covariate in order to study age related effects on GM volume differences.

An exploratory whole brain analysis was performed with a threshold for statistical significance of $P<0.001$ without correction for multiple comparisons. Clusters were considered significant using the family-wise error (FWE) threshold of $P_{\mathrm{FWE}}<0.05$, corrected for multiple comparisons based on the random field theory. In addition, post-hoc regions of interest (ROI) analyses were performed using mask images for these regions from the SPM8 Anatomy Toolbox. Significant group differences are reported at $P_{\mathrm{Scv}}<0.05$ (small volume correction, i.e., family wise error correction within the search volume of $8 \mathrm{~mm}$ ). The $\mathrm{x}, \mathrm{y}$, and $\mathrm{z}$ coordinates of areas displaying significant whole brain or ROI group differences in GM were identified using the Brede Database, ${ }^{25}$ the Nonlinear Yale MNI to Talairach Conversion Algorithm, ${ }^{26}$ and the SPM Anatomy Toolbox. 
Statistical analysis of the behavioral data was performed using the Statistical Package for Social Sciences version 22 (SPSS, Chicago). The numerical variables were described as means and standard deviations and the categorical variables as frequencies and percentages. Group comparisons were performed for demographical and clinical variables, as well as for the cognitive variables, using an analysis of variance with generalized linear model with Tukey correction for multiple testing when data distribution was normal and a Kruskal-Wallis test was used for non-normal distributions. Categorical variables were compared with Chi-square test. A $P$ value $<0.05$ was considered significant.

\section{Results}

\section{Demographics}

Table 1 shows the demographic and clinical characteristics of the 4 groups. Patients in group 1 were significantly younger than those in the three other groups and received more years of formal education than patients in group 3 and 4. Patients from group 4 showed more disease-related speech abnormalities than those in the other three groups and more frequent and more severe hallucinations than patients in group 1 and 2. The four groups did not significantly differ with respect to disease duration, severity of motor symptoms, postural instability, frequency of dyskinesia, antiparkinsonian medication, frequency of REM sleep behavioral disorders, frequency of vascular risk factors, and severity of depressive symptoms. Dementia was more frequent in group 4 than in the other groups, as was a reduced level of autonomy. Group 3 showed more symptoms of persistent and avoidance anxiety and more symptoms of apathy than group 1 .

\section{Neuropsychological data}

Table 2 shows the neuropsychological data of the 4 patient groups. Patients from group 1 and 2 were cognitively intact, with high/normal levels of performance in group 1 for all cognitive domains, and only slight slowing of information processing speed and fewer animals named in group 2. Group 3 scored lower on global cognition (i.e., MMSE and MDRS) than the cognitively intact groups (i.e., group 1 and 2 ), yet still within the normal range. Patients in group 3 further scored lower on tests measuring attention, working memory, episodic verbal memory (except recognition), executive functions, language and visuospatial functions. Patients in group 4 showed impaired global cognition and severe deficits in all cognitive domains. 


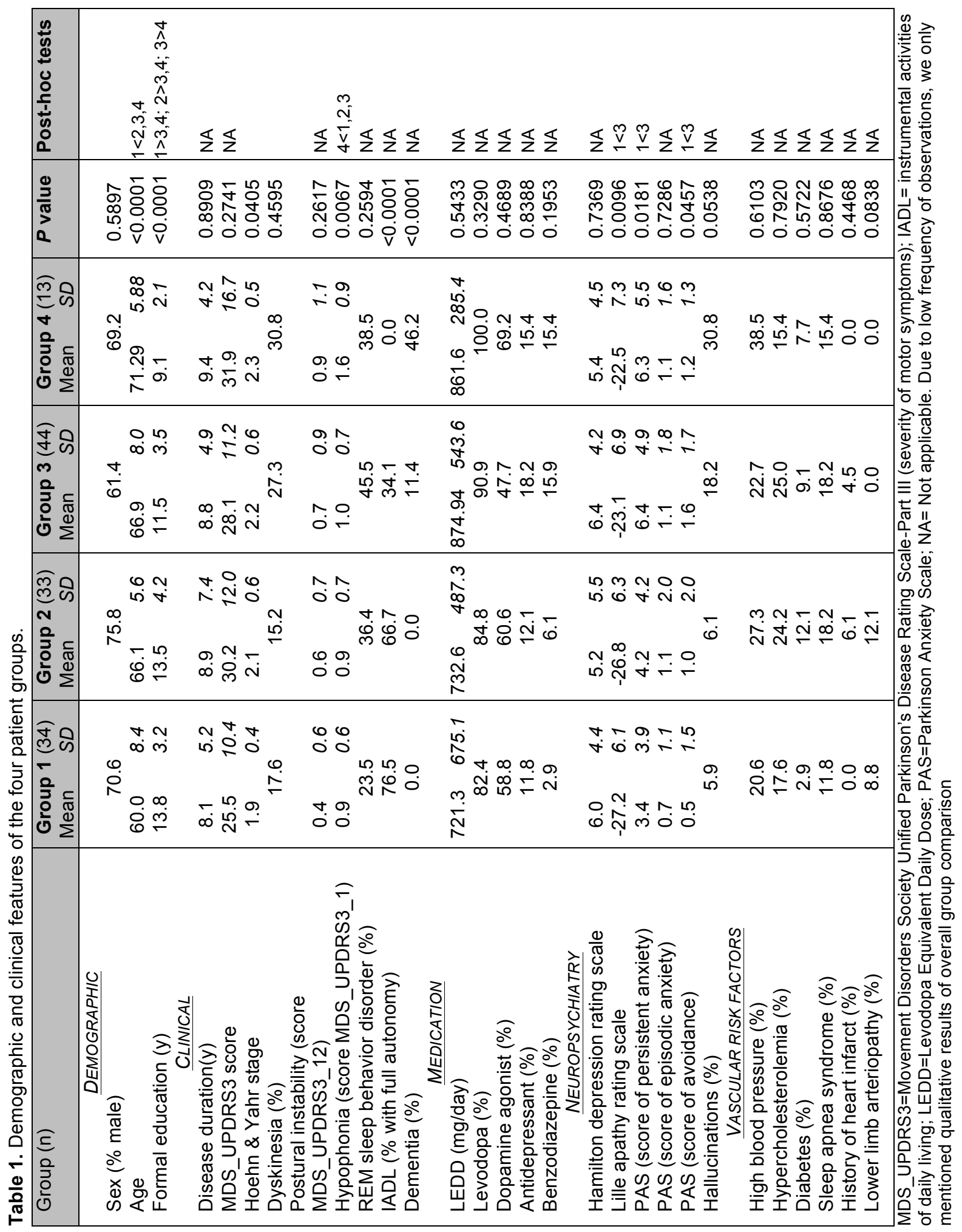




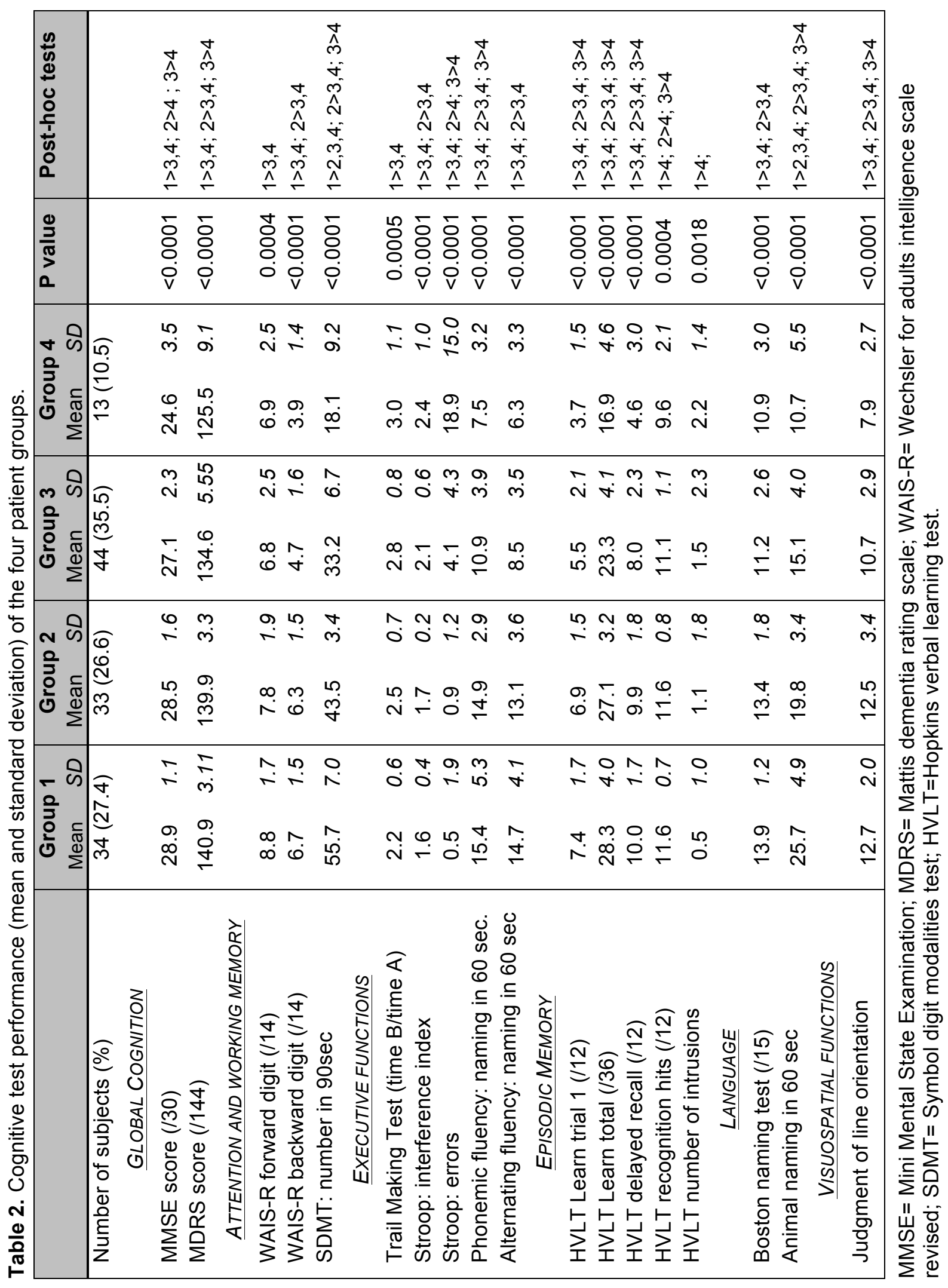




\section{VBM results}

Figure 1 demonstrates the areas where group $2(A)$, group $3(B)$, and group $4(C)$ showed differences in grey matter volume compared to group 1 . The reversed contrasts with group 1 or comparisons among group 2, 3 and 4 did not yield any significant results. All significant results are corrected for effects of center, gender and level of education.

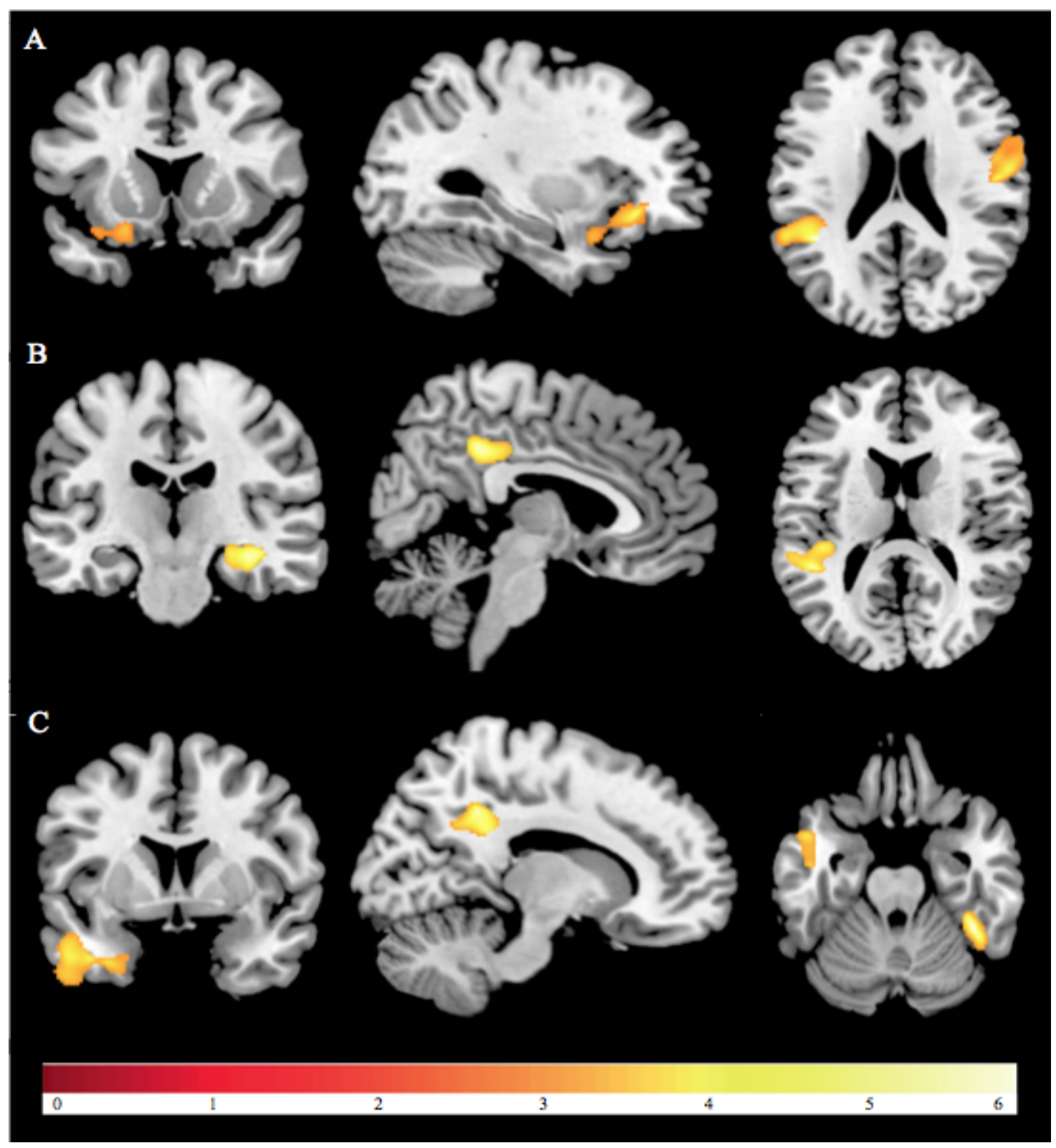

Figure 1. VBM group comparisons of grey matter (GM) density with reduced A) left superior temporal, left orbitofrontal, right inferior frontal, and left inferior parietal lobe GM density in group 2 vs. group 1; B) right hippocampal, right amygdala, posterior cingulate, and left inferior parietal GM density in group 3 vs. group 1; C) left medial/ inferior temporal and right posterior cingulate GM density in group 4 vs. group 1 . The threshold was set at $P_{\mathrm{FWE}}<0.05$, corrected for multiple comparisons based on the random field theory. The color scale represents the T-value corresponding to the significant cluster. 
Compared to group 1, group 2 shows more atrophy in the temporal (Brodmann area (BA) 22, 38) and parietal (BA 3a, 3b, 40) lobe areas and the middle, orbital and inferior frontal gyrus (BA 11, 44, 47). Group 3 showed more atrophy than group 1 in the temporal (BA 22, 41) and parietal (BA 31, 40) lobe areas as well as in the right hippocampus and right amygdala. Small volume corrected region-of-interest (ROI) analyses showed reduced grey matter density in the hippocampus (mainly CA2) and the amygdala (both bilaterally) in group 3 compared to group 1 (Figure 2). Group 4 showed more grey matter atrophy in the temporal (BA 20, 21, 36, 38) and parietal (BA 23, 31) lobe areas, compared to group 1. Table 3 contains information regarding the location and $\mathrm{MNI}$ coordinates of regions that yielded significant results.

After including age as a covariate into the model as well, no differences in grey matter volume between any of the groups remained significant. A more liberal statistical threshold of $P<0.005$ revealed more grey matter atrophy in group 4 compared to group 1 within the right posterior cingulate gyrus (BA 31) and right precuneus (BA 7), and a trend within the left medial temporal pole (BA 38, $P_{\mathrm{FWE}}=0.052$ ). See Table 3 for specific details about location and MNI coordinate

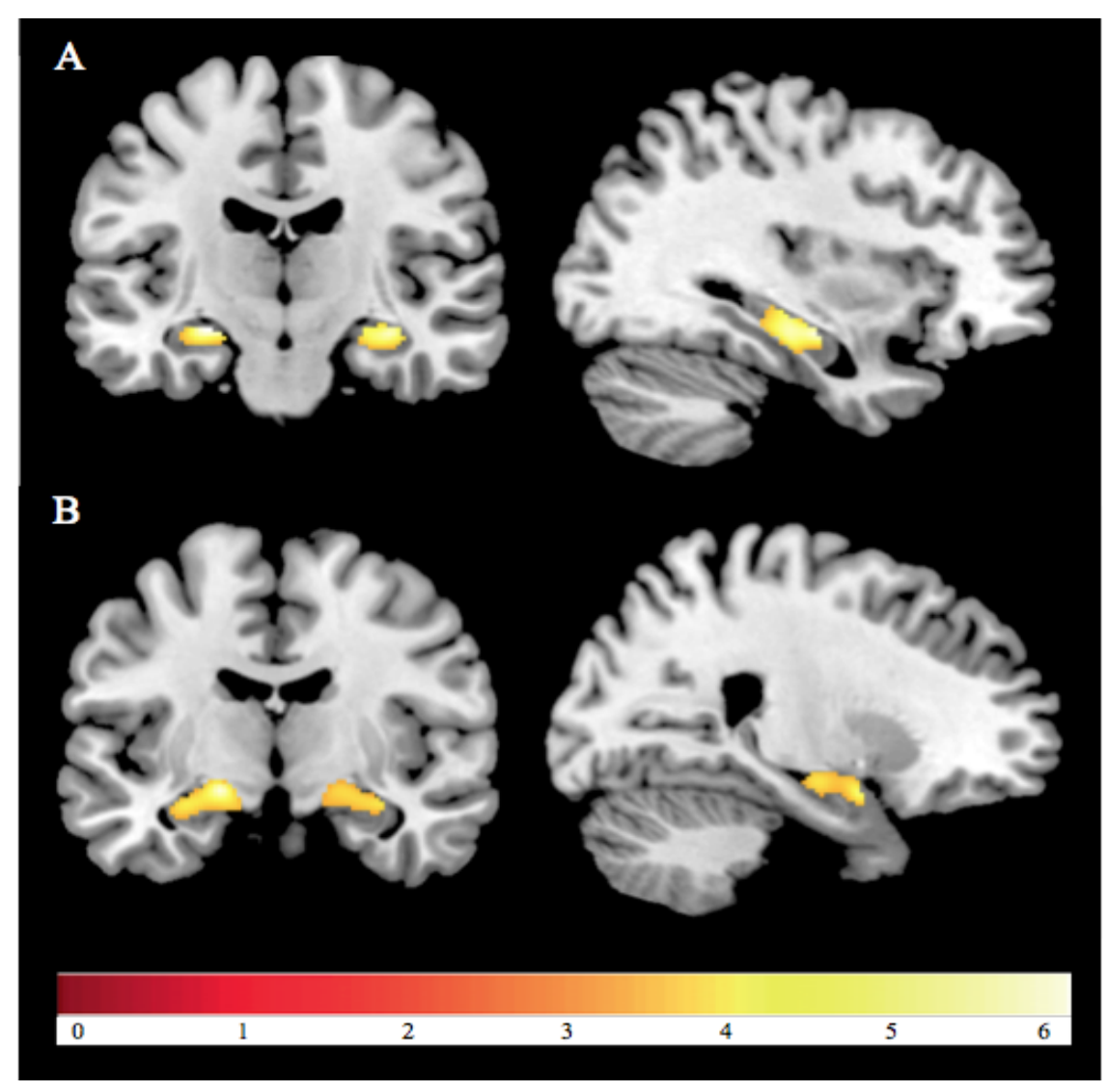

Figure 2. ROI analysis for the bilateral hippocampus (A) and bilateral amygdala (B) showing reduced grey matter density in group 3 vs. group 1. The threshold was set at $P_{\mathrm{FWE}}<0.05$, corrected for multiple comparisons using small volume correction (svc). The color scale represents the T-value corresponding to the significant cluster. 


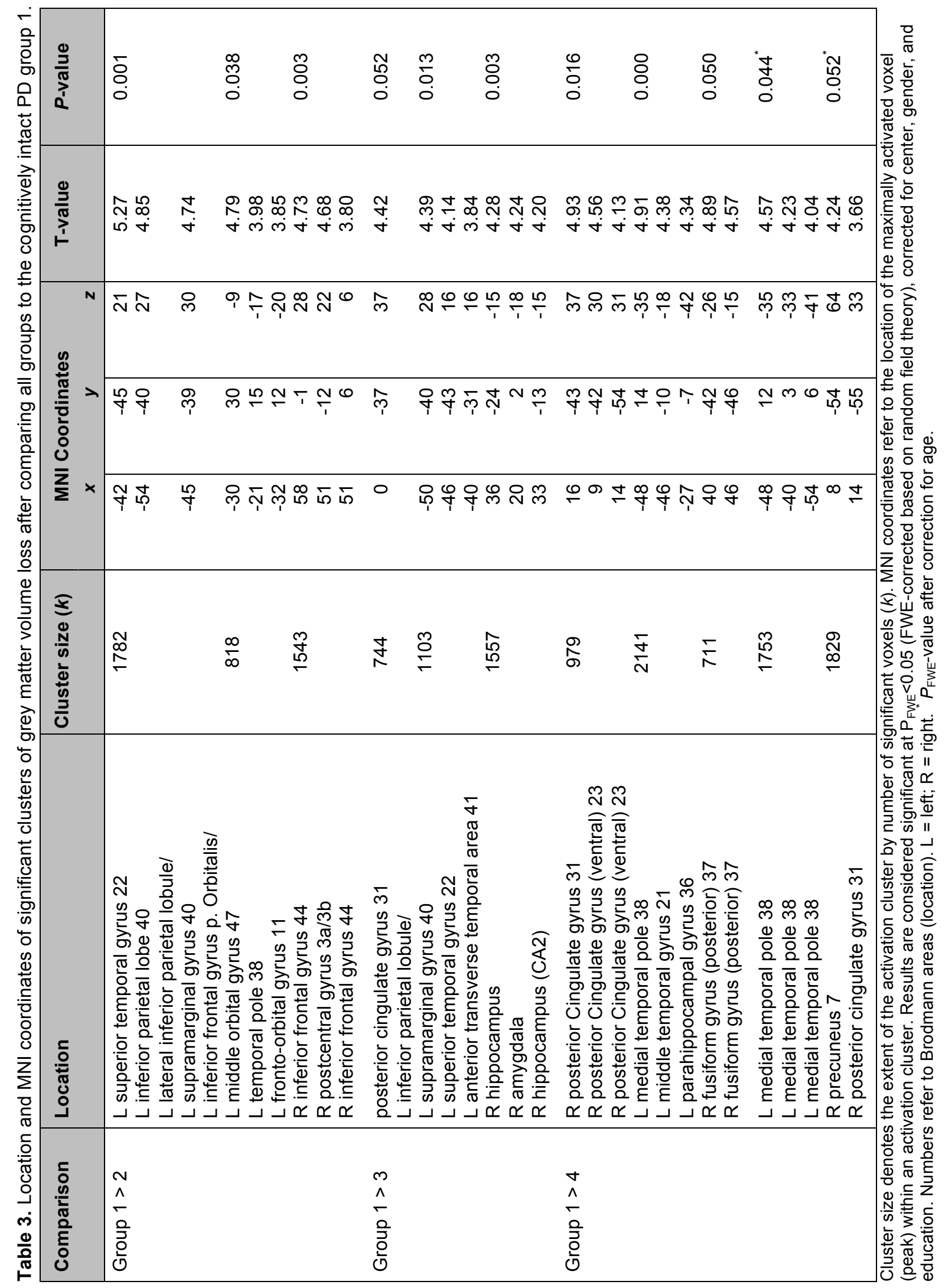




\section{Discussion}

In a previous study, we found evidence for the heterogeneity of cognitive decline in Parkinson's disease (PD) and identified five cognitive profiles. ${ }^{7}$ In the present study, we aimed to identify the neuroanatomical correlates of four of these cognitive phenotypes using structural MRI and analyzed differences in grey matter (GM) density using optimized voxel-based morphometry (VBM-Dartel). The results confirmed our hypothesis that all groups would show reduced regional GM density compared to the cognitively intact group. Our results gave no indication for a regional progressive severity gradient of GM atrophy. Instead, we observed an extension of affected areas indicating more widespread pathological changes associated with progressive cognitive decline.

Probably the most remarkable result concerns the amount of GM atrophy already visible in PD patients that were still cognitively intact (group 2), with only slight mental slowing and a lower score for semantic fluency. These patients showed a similar pattern of GM atrophy that is characteristic for PD patients with dementia, including the parietal-temporal lobe, prefrontal cortex, and posterior cingulate., ${ }^{4,27,28}$ These results provide important support for the view that slowed mental speed and its corresponding pattern of GM atrophy can be considered as a marker of early cognitive decline in PD. It also emphasizes the need to clinically follow those patients and offer them treatment options in an early phase in order to prevent further cognitive decline.

Patients in group 3 represent a cognitive profile that fits largely with the criteria for dysexecutive mild cognitive impairment $(\mathrm{MCl})$, showing cognitive deficits in the field of attention and executive functions. ${ }^{29}$ Similar to group 2, patients from group 3 showed reduced GM volume in the parietal-temporal lobe and posterior cingulate. In addition, we observed a significant increase in GM atrophy in the bilateral hippocampus (CA2 region) and amygdala compared to group 1. This is in line with results from Weintraub and colleagues, ${ }^{4}$ who suggest that hippocampal neurodegeneration can be associated with early cognitive decline in PD-MCl.

Group 4 is characterized by global cognitive decline and severe cognitive deficits in multiple cognitive domains, including verbal episodic memory and visuospatial deficits. VBM analyses revealed an extension of the atrophy pattern seen in the other groups, with additional atrophy in the left medial temporal lobe and left parahippocampal gyrus, yet significant differences in GM density were limited to group 1. This extension to medial temporal areas is in line with other VBM studies, ${ }^{4,27}$ 
and possibly reflects more widespread Lewy body pathology as well as a marker for conversion to PD dementia (PDD). Within group 4, six patients already met the criteria for mild PDD, while the remaining patients could be classified as multiple domains $\mathrm{MCl}^{29}$ Another peculiarity within the clinical profile of this group was the high rate of visual hallucinations. The association between visual hallucinations and severe cognitive decline ${ }^{30}$ as well as a higher risk for developing dementia, ${ }^{31}$ has been related to both Lewy body pathology in the temporal lobe ${ }^{32}$ and cholinergic deficits. $^{33}$

Alternatively, left medial temporal lobe atrophy and GM loss in the parahippocampal gyrus have been shown to be consistent neurostructural biomarkers for conversion to Alzheimer's disease (AD). ${ }^{27,34}$ In addition, autopsy studies revealed that a substantial number of PD patients appear to have neuropathological changes related to $A D$ in addition to diffuse Lewy body pathology ${ }^{35-37}$ As such, more advanced stages of cognitive decline in PD may reflect an interaction of different neurodegenerative processes such as comorbid AD-related neurodegenerative changes.$^{38}$ Moreover, the combination of amyloid- and tau-related pathological changes and limbic and cortical Lewy body abnormalities appear to be the strongest correlate of dementia in $\mathrm{PD}^{39}$ Other factors, such as neuroinflammation, synaptic pathological changes, and neurotransmitter and vascular changes may be involved as well. In our study we did not find evidence for a contribution of vascular changes to cognitive decline, neither did we investigate the role of different neurotransmitter systems here. However, some evidence has been reported that dopaminergic deficits in frontostriatal circuits may be more involved in milder executive-attention profiles of cognitive decline, ${ }^{5}$ while cholinergic deficits and Lewy body abnormalities in non-dopaminergic cortical structures are thought to contribute to more severe cognitive decline ${ }^{40}$ including memory encoding deficits and visuospatial deficits as seen in PDD.

Age appeared to have a substantial influence on our results. This is not surprising, given that age is the most important risk factor for cognitive disorders and for developing dementia in the general population. Our groups were defined through a data-driven approach based on cognitive performance. As expected, the progressive decline in cognition was accompanied by an increase in age and as a result, all groups were significantly older than the cognitively intact group (group 1). We aimed to reveal disease-related atrophy associated with cognitive status independent of age by adding age as covariate into the model. However, differences 
in GM atrophy were no longer visible when comparing group 2 and 3 with group 1, suggesting that age was the main predictor of cognitive decline in these groups. Moreover, age at onset and disease duration did not differ between groups, supporting evidence from previous studies that age at evaluation is a more important predictor of rate of cognitive decline than age at onset or disease duration (for review see ref $\left[{ }^{41}\right]$ ). The group with severe cognitive deficits (group 4) still showed increased regional GM atrophy compared to group 1 after correction for age, possibly revealing effects of the disease process in addition to effects of aging. Levy ${ }^{41}$ proposed a model for the relationship between PD and advanced aging, stating that advanced age is associated with a faster rate of motor progression, decreased levodopa responsiveness, more severe gait and postural impairments, reduced autonomy, more severe cognitive impairment and a higher risk for developing dementia in PD. The model further suggests a substantial role for aging in the pathogenesis of PD, by means of a biologic interaction between the effects of the disease process and aging in non-dopaminergic structures, which may indeed denote the involvement of monoaminergic and cholinergic structures in more advanced stages of PD.

Our study has a number of limitations. First, due to our study design (datadriven approach in order to identify cognitive phenotypes in PD), we did not include a healthy control group and group 1 (cognitively intact PD patients) was considered as our reference group. Hence, we were not able to identify early GM loss in this group compared to healthy controls. Also, with our design we were not able to disentangle the absolute contribution of disease to cognitive decline independently of age. Age appeared to be an overpowering factor in the model, possibly masking other factors. Secondly, the number of subjects in the original clusters 4 and 5 was low, probably because we did not include subjects with moderate or severe dementia in order to limit imaging artifacts and missing data due to misunderstanding of instructions. However, despite collapsing these two clusters, the remaining group 4 still remained very small $(n=13)$. Results for this group after correction for age would probably have reached the level of significance without a more liberal threshold if the sample had been larger. Thirdly, patients were recruited from tertiary referral centers, making the sample possibly not representative for the general population. However, the advantage of recruiting from movement disorders centers is that reliable records are provided with PD specific information regarding in- and exclusion criteria. Fourthly, spatial normalization and image segmentation in VBM is fully automated, which may lead to difficulties in detecting subtle changes/atrophy in areas that show high 
variability among elderly and diseased groups (e.g., the hippocampus) and may lead to misclassification of voxels due to reduced tissue contrast. However, the use of a strict threshold for significance reduced the possibility for such errors substantially. Moreover, the use of automated whole brain analysis methods for evaluating brain structure in diseased brains is less prone to subjectivity associated with ROI based methods and is not restricted by a priori assumptions about specific regions of interest. Another important aspect concerns the use of a cross-sectional design, which limited us to merely describe the clinical characteristics and neural substrates of the separate cognitive profiles. A follow-up of the identified groups would be essential to explore differences in disease progression. Moreover, this may reveal the extent to which neural substrates associated with early markers of cognitive impairment (e.g., slowed mental speed) can be used as a predictor of severe cognitive decline and conceivable conversion to dementia. Also, theories about underlying pathology remain speculative here and need to be confirmed in future studies. However, an important strength of our study design relates to the use of a data-driven approach. Groups were not previously defined based on diagnostic criteria for mild cognitive impairment and PDD, which enabled us to reveal a phenotype reflecting intact cognition with slight mental slowing, despite clear GM abnormalities compared to PD patients who showed no cognitive deficits at all. The combination of a data-driven approach to define groups and whole brain analysis turned out to be very useful for identifying markers of early cognitive decline in PD.

In conclusion, our results revealed a pattern of cerebral grey matter atrophy indicating more widespread pathological changes associated with progressive cognitive decline in PD. Remarkably, this pattern could already be detected in patients with intact cognition and may therefore be considered as a marker of early cognitive decline in PD. Moreover, slowed mental speed is probably a first, yet crucial, sign of cognitive deterioration in PD and regular MRI assessments could inform clinicians about the progressive course of cerebral atrophy associated with cognitive impairment. Although age appeared to be an important predictor of early cognitive decline in PD patients, we found GM abnormalities unrelated to age in patients with severe cognitive deficits. This may resemble effects of disease processes in addition to aging, such as more widespread Lewy body pathology potentially with comorbid AD pathology. Longitudinal studies are needed to disentangle the specific contribution of neurochemical and neuropathological factors related to the course of cognitive decline in PD. 


\section{REFERENCES}

1. Svenningsson $P$, Westman E, Ballard C, Aarsland D. Cognitive Impairment in Patients with Parkinson's Disease: Diagnosis, Biomarkers, and Treatment. The Lancet Neurology 2012;11:697-707.

2. Foltynie T, Brayne CE, Robbins TW, Barker RA. The Cognitive Ability of an Incident Cohort of Parkinson's Patients in the Uk. The Campaign Study. Brain 2004;127:550560.

3. Janvin C, Aarsland D, Larsen JP, Hugdahl K. Neuropsychological Profile of Patients with Parkinson's Disease without Dementia. Dementia and geriatric cognitive disorders 2003;15:126-131.

4. Weintraub D, Doshi J, Koka D, Davatzikos C, Siderowf AD, Duda JE, et al. Neurodegeneration across Stages of Cognitive Decline in Parkinson Disease. Archives of Neurology 2011;68:1562-1568.

5. Kehagia AA, Barker RA, Robbins TW. Neuropsychological and Clinical Heterogeneity of Cognitive Impairment and Dementia in Patients with Parkinson's Disease. The Lancet Neurology 2010;9:1200-1213.

6. Dujardin K, Leentjens AF, Langlois C, Moonen AJ, Duits AA, Carette AS, et al. The Spectrum of Cognitive Disorders in Parkinson's Disease: A Data-Driven Approach. Movement Disorders 2013;28:183-189.

7. Dujardin K, Moonen AJ, Behal H, Defebvre L, Duhamel A, Duits A, et al. Cognitive Disorders in Parkinson's Disease: Confirmation of a Spectrum of Severity. Parkinsonism and Related Disorders 2015; 21: 1299-1305.

8. Gibb W, Lees A. The Relevance of the Lewy Body to the Pathogenesis of Idiopathic Parkinson's Disease. J Neurol, Neurosur Ps 1988;51:745-752.

9. Morris JC. The Clinical Dementia Rating (Cdr): Current Version and Scoring Rules. Neurology 1993;43:2412-2414.

10. Emre M, Aarsland D, Brown R, Burn DJ, Duyckaerts C, Mizuno Y, et al. Clinical Diagnostic Criteria for Dementia Associated with Parkinson's Disease. Movement Disorders 2007;22:1689-1707.

11. Goetz CG, Tilley BC, Shaftman SR, Stebbins GT, Fahn S, Martinez-Martin P, et al. Movement Disorder Society-Sponsored Revision of the Unified Parkinson's Disease Rating Scale (Mds-Updrs): Scale Presentation and Clinimetric Testing Results. Movement Disorders 2008;23:2129-2170.

12. Hamilton M. A Rating Scale for Depression. Journal of neurology, neurosurgery, and psychiatry 1960;23:56.

13. Leentjens AF, Dujardin K, Pontone GM, Starkstein SE, Weintraub D, Martinez-Martin P. The Parkinson Anxiety Scale (Pas): Development and Validation of a New Anxiety Scale. Movement Disorders 2014;29:1035-1043.

14. Sockeel P, Dujardin K, Devos D, Deneve C, Destée A, Defebvre L. The Lille Apathy Rating Scale (Lars), a New Instrument for Detecting and Quantifying Apathy: Validation in Parkinson's Disease. Journal of Neurology, Neurosurgery \& Psychiatry 2006;77:579-584.

15. Lawton MP, Brody EM. Assessment of Older People: Self-Maintaining and Instrumental Activities of Daily Living. The Gerontologist 1969;9:179-186.

16. Folstein MF, Folstein SE, McHugh PR. "Mini-Mental State": A Practical Method for Grading the Cognitive State of Patients for the clinician. J Psy Res 1975; 12:189-198. 
17. Mattis S. Mental Status Examination for Organic Mental Syndrome in the Elderly Patient, In : L. Bellak, T. L. (Eds). Geriatric psychiatry, Grune and Stratton, New York 1976;77-121.

18. Wechsler D. Wais-R: Wechsler Adult Intelligence Scale, Revised. 1986.

19. Smith A. Symbol Digits Modalities Test, Western Psychological Services, Los Angeles. 1982.

20. Reitan R. Tmt, Trail Making Test a \& B, South Tucson. 1992.

21. Brandt J, Benedict R. Hopkins Verbal Learning Test-Revised, Psychological Assessment Resources, Lutz. FI, 2001.

22. Graves RE, Bezeau SC, Fogarty J, Blair R. Boston Naming Test Short Forms: A Comparison of Previous Forms with New Item Response Theory Based Forms. Journal of Clinical and Experimental Neuropsychology 2004;26:891-902.

23. Benton AL, Varney NR, Hamsher KS. Visuospatial Judgment: A Clinical Test. Archives of Neurology 1978;35:364-367.

24. Ashburner J. A Fast Diffeomorphic Image Registration Algorithm. Neuroimage 2007;38:95-113.

25. Nielsen FA. The Brede Database: A Small Database for Functional Neuroimaging. 2003.

26. Lacadie CM, Fulbright RK, Rajeevan N, Constable RT, Papademetris X. More Accurate Talairach Coordinates for Neuroimaging Using Non-Linear Registration. Neuroimage 2008;42:717-725.

27. Burton EJ, McKeith IG, Burn DJ, Williams ED, O'Brien JT. Cerebral Atrophy in Parkinson's Disease with and without Dementia: A Comparison with Alzheimer's Disease, Dementia with Lewy Bodies and Controls. Brain 2004;127:791-800.

28. Song SK, Lee JE, Park HJ, Sohn YH, Lee JD, Lee PH. The Pattern of Cortical Atrophy in Patients with Parkinson's Disease According to Cognitive Status. Movement Disorders 2011;26:289-296.

29. Litvan I, Goldman JG, Tröster AI, Schmand BA, Weintraub D, Petersen RC, et al. Diagnostic Criteria for Mild Cognitive Impairment in Parkinson's Disease: Movement Disorder Society Task Force Guidelines. Movement Disorders 2012;27:349-356.

30. Aarsland D, Andersen K, Larsen JP, Perry R, Wentzel-Larsen T, Lolk A, et al. The Rate of Cognitive Decline in Parkinson Disease. Archives of Neurology 2004;61:19061911.

31. Aarsland D, Andersen K, Larsen JP, Lolk A. Prevalence and Characteristics of Dementia in Parkinson Disease: An 8-Year Prospective Study. Archives of Neurology 2003;60:387-392.

32. Harding A, Broe G, Halliday G. Visual Hallucinations in Lewy Body Disease Relate to Lewy Bodies in the Temporal Lobe. Brain 2002;125:391-403.

33. Perry EK, Marshall E, Kerwin J, Smith CJ, Jabeen S, Cheng AV, et al. Evidence of a Monoaminergic-Cholinergic Imbalance Related to Visual Hallucinations in Lewy Body Dementia. Journal of neurochemistry 1990;55:1454-1456.

34. Ferreira LK, Diniz BS, Forlenza OV, Busatto GF, Zanetti MV. Neurostructural Predictors of Alzheimer's Disease: A Meta-Analysis of Vbm Studies. Neurobiology of aging 2011;32:1733-1741.

35. Kalaitzakis M, Pearce RB. The Morbid Anatomy of Dementia in Parkinson's Disease. Acta Neuropathologica 2009;118:587-598. 
36. Kurosinski P, Guggisberg M, Götz J. Alzheimer's and Parkinson's DiseaseOverlapping or Synergistic Pathologies? Trends in molecular medicine 2002;8:3-5.

37. Jellinger K, Seppi K, Wenning G, Poewe W. Impact of Coexistent Alzheimer Pathology on the Natural History of Parkinson's Disease. Journal of neural transmission 2002;109:329-339.

38. Sabbagh MN, Adler CH, Lahti TJ, Connor DJ, Vedders L, Peterson LK, et al. Parkinson's Disease with Dementia: Comparing Patients with and without Alzheimer Pathology. Alzheimer disease and associated disorders 2009;23:295.

39. Compta Y, Parkkinen L, O'Sullivan SS, Vandrovcova J, Holton JL, Collins C, et al. Lewy-and Alzheimer-Type Pathologies in Parkinson's Disease Dementia: Which Is More Important? Brain 2011;134:1493-1505.

40. Williams-Gray C, Foltynie T, Brayne C, Robbins T, Barker R. Evolution of Cognitive Dysfunction in an Incident Parkinson's Disease Cohort. Brain 2007;130:1787-1798.

41. Levy G. The Relationship of Parkinson Disease with Aging. Archives of neurology 2007;64:1242-1246. 



\section{CHAPTER 6}

\section{Modeling DEPRESSION IN PARKINSON'S DISEASE: DISEASE-SPECIFIC AND}

NON-SPECIFIC RISK FACTORS

Neurology, 81: 1036-1043, 2013

Albert F.G. Leentjens, Anja J.H. Moonen, Kathy Dujardin, Laura Marsh, Pablo Martinez-Martin, Irene H. Richard, Sergio E. Starkstein, Sebastian Köhler 


\section{ABSTRACT}

Objective: To construct a model for depression in Parkinson's disease (PD) and to study the relative contribution of PD-specific and non-specific risk factors to this model.

Methods: Structural equation modeling of direct and indirect associations of risk factors with the latent depression outcome using a cross-sectional dataset of 342 PD patients.

Results: A model with acceptable fit was generated that explained $41 \%$ of the variance in depression. In the final model, three PD-specific variables (increased disease duration, more severe motor symptoms, the use of levodopa) and six nonspecific variables (female sex, a previous history of anxiety and/or depression, a family history of depression, worse functioning on activities of daily living, and worse cognitive status) were maintained and significantly associated with depression. Nonspecific risk factors had a three times higher influence in the model than PD-specific risk factors.

Conclusion: In this cross-sectional study, we showed that non-specific factors may be more prominent markers of depression than PD-specific factors. Accordingly, research on depression in PD should focus not only on factors associated with, or specific for PD, but should examine a wider scope of factors including also general risk factors for depression, not specific for PD, in their design. 


\section{INTRODUCTION}

Clinically relevant depressive symptoms are present in 35\% of patients with Parkinson's disease (PD) and depression has been identified as the most important determinant of quality of life in these patients. ${ }^{1-3}$ Depressive disorders, in the general population as well as in PD patients, develop in the context of multiple interacting risk and protective factors. These factors may or may not be related to PD. In the general population, longitudinal studies have shown that risk factors for depression include female sex, higher age, being single, a low level of education, physical disease, a personal or family history of depression, cognitive impairment, smoking, and alcohol. Moreover, personal circumstances, such as early childhood adversity, personality traits and coping, and recent positive and negative life events also play a role. ${ }^{4-7}$ In cross-sectional studies, several PD-specific risk factors for depression have been identified, including more severe motor symptoms, longer disease duration, more advanced disease stage, greater limitations in disease-related ADL, higher levodopa equivalent daily dose (LEDD), and the presence of non-motor symptoms such as hallucinations, sleep disturbances and dysautonomia. ${ }^{8-11} \mathrm{Few}$ studies have studied the role of general risk factors for depression in PD.

The objective of this study was to construct a model for depression in PD, and to compare the relative contribution of PD-specific and non-specific markers to this model.

\section{METHODS}

The present study was performed on the database from a cross-sectional multicenter study on anxiety disorders in PD that was conducted in 2008 and 2009, the results of which have been described in two papers published earlier. ${ }^{12,13}$

\section{Population}

The database includes 342 patients with PD, diagnosed according to the Queens Square Brain Bank clinical criteria. ${ }^{14}$ Subjects, recruited from the Movement Disorders clinics and the Neurology and Psychiatry clinics of six centers in the United States, Europe, and Australia, underwent a comprehensive neurological and neuropsychiatric assessment. Patients with neurodegenerative disorders other than PD were excluded. Patients with clinically relevant cognitive symptoms were also excluded. This was operationalized as a Mini-Mental State Examination (MMSE) 
score $<26$, following the recommendation of a Movement Disorders Society (MDS) Task Force. ${ }^{15,16}$ All types of neurological and psychopharmacological medication were allowed. Patients treated with deep brain stimulation (DBS) were excluded.

Approvals, registrations, and patient consents

The study was approved by the local Medical Ethics Committees of all participating institutions. Patients gave written informed consent before inclusion in the study.

\section{Assessment}

Demographic and disease-related variables were assessed during an unstructured clinical interview. Motor function, disease-related decline in ADL, and complications of therapy were assessed with the Unified Parkinson's Disease Rating Scale (UPDRS). ${ }^{17}$ Disease stage was assessed with the Hoehn \& Yahr staging system. ${ }^{18}$ Cognitive functions and instrumental ADL were assessed with the MMSE and Lawton Instrumental ADL (IADL) scale. ${ }^{15,19}$ Depressive symptom severity was quantified with the 17-item Hamilton Depression Rating Scale (HAMD) ${ }^{20}$; anxiety was assessed with the clinician-rated Hamilton Anxiety Rating Scale (HARS). ${ }^{21}$ Patients with 'on/off' fluctuations were only assessed during 'on' states, as advised by an MDS task force. ${ }^{22}$ Also in accordance with MDS task force recommendations, we followed an 'inclusive' approach to rating symptoms, meaning that symptoms were scored as observed or reported, irrespective of their assumed etiology. ${ }^{22}$ The presence of DSMIV-defined depressive and anxiety disorders was determined using the Mini International Neuropsychiatric Inventory (MINI, a structured interview for DSM-IV disorders) sections for depression (A, B) and anxiety (D, E, F, H) ${ }^{23}$

\section{Statistical analysis}

Structural equation modeling (SEM) was performed in Mplus 7 (Múthen \& Múthen, Los Angeles) with the aim of identifying the most parsimonious model (in terms of model fit and number of included parameters) that still accounted for a substantial part of the variance in the depression outcome. Independent variables were chosen on the basis of their known contribution to depression in the general population and in PD from the available literature. An exploratory correlation analysis was carried out between all potential parameters in order to discover collinearity. If the Pearson correlation co-efficient ' $r$ ' between two potential parameters was both significant $(P<$ 0.005 after correction for multiple testing) and larger than 0.40 , a decision was made to include only one of these parameters in the model, where general markers would 
have preference over PD-specific markers. An initial theoretical Multiple Indicators Multiple Causes (MIMIC) model was constructed with the remaining parameters. Since the MIMIC model included continuous, binary and ordered categorical variables, a mean and variance corrected weighted-least-squares estimator was used.

For the sake of the analysis, on the measurement side of the model, a latent variable of 'depression' was constructed by regressing the 17 items of the HAMD on a single continuous latent variable. A 'latent' variable in SEM is a hypothetical construct, which is not measured directly, but estimated in the model from several measured variables, in this case from the individual HAMD items. Compared to using observed HAMD total scores, this approach has the advantage that only shared variances among the items contribute to the depression factor, while non-shared (unique) variance is regarded as measurement error, resulting in a purer operationalization of the latent ("true") depression construct.

For the structural part of the model, direct and indirect paths of the variables theorized to influence the depression outcome were specified in an initial model (model 1). Model fit was primarily assessed by inspecting the Root Mean Squared Error of Approximation (RMSEA) and the Comparative Fit Index (CFI). For the RMSEA, scores $\leq 0.05$ indicate good fit, and scores $\leq 0.08$ indicate acceptable fit. The CFI ranges from 0 to 1 with scores $\geq 0.95$ indicating good fit, and scores $\geq 0.90$ indicating acceptable fit. ${ }^{24}$ Since the chi-square test is known to become positively biased with increasing sample size, this measure was not used to assess model fit. ${ }^{24}$

Based on model fit, the model was re-specified via a number of consecutive steps. First, paths that did not contribute substantially $(P>0.10)$ to the model were removed in a backward one-to-one fashion, starting with the model with the path with the highest p-value, resulting in a second model (model 2). Next, modification indices (Mls) were inspected to explore whether the model could be improved by specifying additional paths among the remaining variables. Additional paths were included based on the Mls and substantive interpretation. Finally, Mls were inspected for correlated errors (residual correlation) among variables whose specification might improve the model. This resulted in the final model.

In a last step, we examined whether PD-specific variables and PD non-specific variables contributed equally to the depression outcome. Since a direct comparison of the joint effect of the manifest variables was not possible due to the correlated nature of individual items, two continuous latent variables (factors) were generated 
and regressed on depression. Their standardized regression coefficients were compared using a Wald test. To have both factors on the same scale, their factor variances were fixed to 1.

\section{RESULtS}

The demographic and disease characteristics of the included sample are listed in Table 1. The study population comprised 207 men and 134 women with an average age of 64.8 years (SD 9.2 years). Based on the MINI, 48 participants (14.1\%) met diagnostic criteria for a current major depressive episode, 19 (5.6\%) met the diagnostic criteria for dysthymia, and 64 (18.8\%) had clinically relevant depressive symptoms (defined here as a score $\geq 12$ ), but did not meet the criteria for major depressive episode or dysthymia.

Results of the exploratory correlation analysis are shown in Suppl. Table 1. Based on the correlations, it was decided to exclude disease stage (Hoehn \& Yahr classification) from the model due to a moderately strong correlation with UPDRS sections 2 and 3 and IADL ( $r=0.60, r=0.46$ and $r=-0.51$ respectively; all $P<0.01)$. UPDRS 4 total score was excluded because of its strong correlation with off-periods $(r=0.66, P<0.01)$. In addition, it was considered more informative to include separate parameters for the presence of 'off-periods' and dyskinesias in the model instead of the single parameter of the UPDRS section 4. It was decided to keep both the UPDRS section 2 (ADL) and the score on the IADL scale in the model, despite moderate correlation $(r=0.46 ; P<0.01)$. This was done because it was thought that the UPDRS section 2 scores PD related ADL, while the IADL scale can be considered a more general measure of personal functioning. It was further decided to keep the UPDRS section 2 score in the model despite its moderate correlation with UPDRS section $3(r=0.51 ; P<0.01)$. 
Table 1. Demographic and disease characteristics of the sample $(n=342)$.

\begin{tabular}{|c|c|c|c|c|}
\hline \multirow{3}{*}{$\begin{array}{l}\text { Variak } \\
\text { Sex }\end{array}$} & & \multirow{3}{*}{$\begin{array}{c}\text { Percentage } \\
39 \\
61\end{array}$} & \multicolumn{2}{|c|}{$\begin{array}{l}\text { Mean (SD) or } \\
\text { median (range) }\end{array}$} \\
\hline & Female & & & \\
\hline & Male & & & \\
\hline \multicolumn{3}{|c|}{ Age (years) } & 64.8 & $(9.2)$ \\
\hline \multicolumn{3}{|c|}{ Duration of PD (years) } & 8.3 & $(5.6)$ \\
\hline \multicolumn{3}{|c|}{ UPDRS section 2 (ADL) } & 11.6 & $(6.8)$ \\
\hline \multicolumn{3}{|c|}{ UPDRS section 3 (motor) } & 26.4 & $(12.4)$ \\
\hline \multicolumn{3}{|c|}{ UPDRS section 4 (complications) } & 3.5 & $(3.5)$ \\
\hline \multicolumn{3}{|c|}{ Hoehn \& Yahr stage (median; range) } & 2 & $(1-5)$ \\
\hline \multicolumn{3}{|c|}{ On/off fluctuations } & & \\
\hline \multicolumn{2}{|c|}{ Major depression } & 14.1 & & \\
\hline \multicolumn{2}{|c|}{ Dysthymia } & 5.7 & & \\
\hline \multicolumn{2}{|c|}{ Previous history depression } & 46.1 & & \\
\hline \multicolumn{2}{|c|}{ Previous history anxiety disorder } & 31.9 & & \\
\hline \multicolumn{2}{|c|}{ Family history depression } & 33.4 & & \\
\hline \multicolumn{2}{|c|}{ Family history anxiety disorder } & 19.4 & & \\
\hline \multicolumn{2}{|c|}{ Family history of PD } & 19.7 & & \\
\hline \multicolumn{2}{|c|}{ HAMD score } & & 7.7 & $(5.9)$ \\
\hline \multicolumn{2}{|c|}{ HARS score } & & 11.3 & $(8.5)$ \\
\hline \multicolumn{2}{|c|}{ IADL score } & & 7 & $(1.5)$ \\
\hline \multicolumn{2}{|c|}{ MMSE score } & & 28.5 & $(1.7)$ \\
\hline \multicolumn{2}{|c|}{ Use of levodopa } & 85.3 & & \\
\hline \multicolumn{2}{|c|}{ Use of dopamine-agonist } & 61.6 & & \\
\hline \multicolumn{2}{|c|}{ Use of antidepressant \# } & 34.4 & & \\
\hline
\end{tabular}

PD = Parkinson's disease; UPDRS = Unified Parkinson's Disease Rating Scale; ADL = Activities of Daily Living; HAMD = Hamilton Depression Scale; HARS = Hamilton Anxiety Rating Scale; IADL = Lawton's Instrumental Activities of Daily Living scale; MMSE = Mini Mental State Examination

\# Antidepressants were used by $56 \%$ of patients suffering from major depressive disorder, by $42 \%$ of patients suffering from dysthymia, and by $30 \%$ of patients not suffering from current depressive symptoms (i.e., the depressive symptoms were in remission or the antidepressant was used for other indications, such as anxiety or sleep problems. 
The initial theoretical model is shown in Figure 1 and specified in Table 2. Nonspecific parameters included in this model were age, sex, cognitive status (MMSE total score), instrumental ADL function (IADL total score), a previous history of depression, a previous history of anxiety, a family history of depression, and a family history of anxiety. PD-specific parameters in the model were disease duration, motor symptom severity (UPDRS section 3 total score), disease-specific ADL (UPDRS section 2 total score), the presence of motor fluctuations (based on UPDRS section 4 ), the presence of dyskinesias (based on UPDRS section 4), the use of levodopa, and the use of a dopamine agonist. This model showed acceptable fit in terms of RMSEA, but poor fit in terms of CFI (RMSEA 0.069 with a $90 \% \mathrm{Cl}=0.064-0.074$; CFI 0.644).

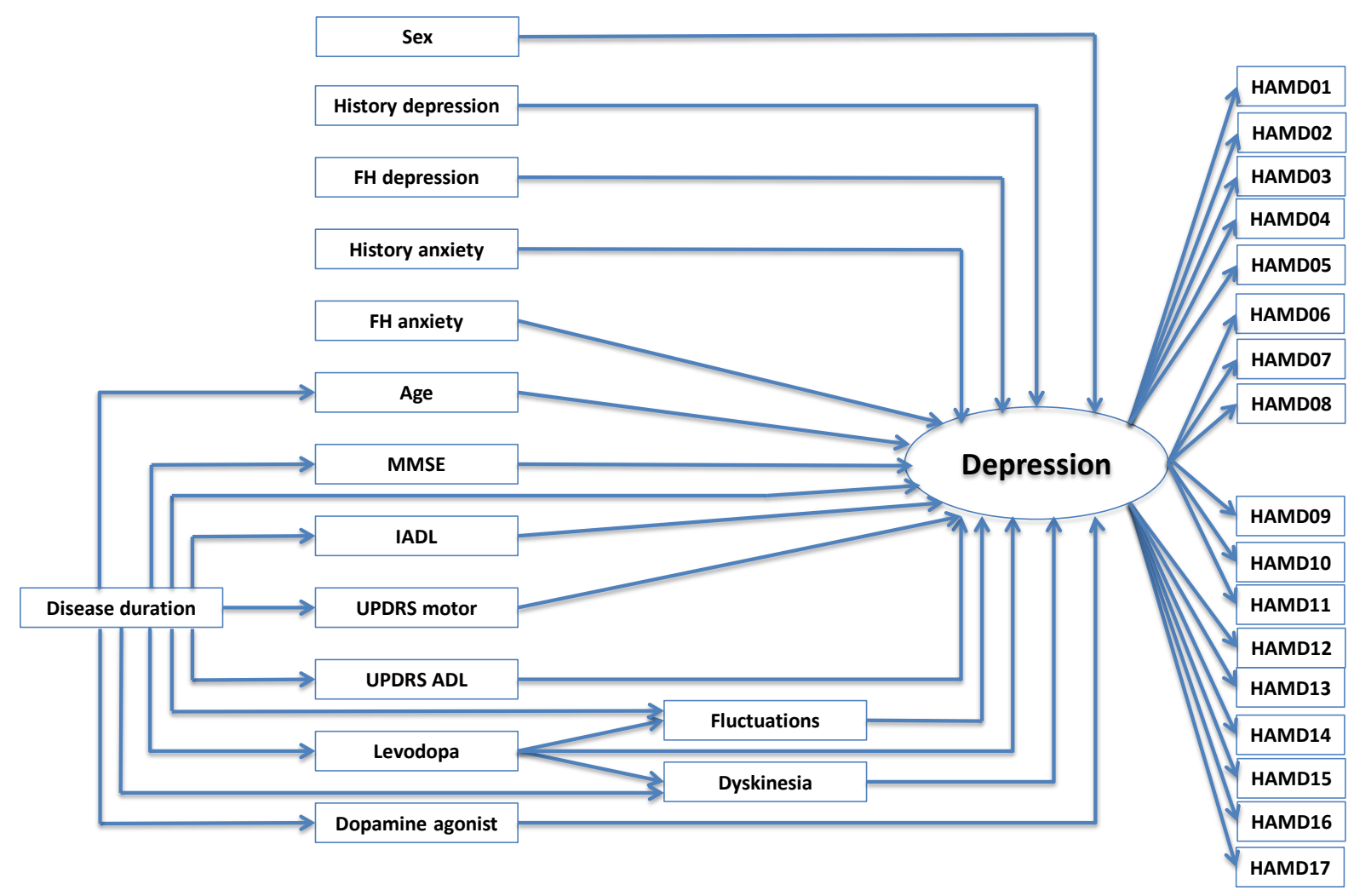

Figure 1. Initial model of depression in PD (model 1). This initial model is the hypothesized model and includes all non-specific and PD-specific variables. Model specifics and regression co-efficients are given in Table 2. $A D L=$ Activities of Daily Living; $F H=$ family history; HAMD = Hamilton Depression Scale; IADL = Lawton's Instrumental Activities of Daily Living scale; MMSE = Mini Mental State Examination; UPDRS = Unified Parkinson's Disease Rating Scale. 
Table 2. Standardized regression coefficients of the initial (theoretical) model including 15 variables (model 1 ).

\begin{tabular}{|c|c|c|c|c|c|c|}
\hline Parameter & $\begin{array}{l}\text { Dependent } \\
\text { variable }\end{array}$ & B & SE & $\beta$ & z-statistic & $P$-value \\
\hline Sex & depression & -0.371 & 0.148 & -0.151 & -2.498 & 0.012 \\
\hline Age & depression & -0.003 & 0.007 & -0.019 & -0.365 & 0.715 \\
\hline History depression & depression & 0.544 & 0.165 & 0.226 & 3.297 & 0.001 \\
\hline History Anxiety & depression & 0.400 & 0.165 & 0.160 & 2.423 & 0.015 \\
\hline FH depression & depression & 0.232 & 0.144 & 0.113 & 1.613 & 0.107 \\
\hline FH anxiety & depression & -0.081 & 0.171 & -0.026 & -0.471 & 0.638 \\
\hline MMSE & depression & -0.177 & 0.043 & -0.244 & -4.162 & $<0.001$ \\
\hline IADL & depression & -0.233 & 0.051 & -0.279 & -4.583 & $<0.001$ \\
\hline Disease duration & depression & -0.096 & 0.026 & -0.445 & -3.734 & $<0.001$ \\
\hline UPDRS motor (s.3) & depression & 0.063 & 0.011 & 0.378 & 5.819 & $<0.001$ \\
\hline UPDRS ADL (s.2) & depression & 0.061 & 0.012 & 0.335 & 5.120 & $<0.001$ \\
\hline Fluctuations & depression & 0.045 & 0.122 & 0.050 & 0.371 & 0.711 \\
\hline Dyskinesias & depression & -0.085 & 0.151 & -0.109 & -0.563 & 0.573 \\
\hline Use of levodopa & depression & 0.328 & 0.275 & 0.349 & 1.194 & 0.232 \\
\hline Use of dopa-agonist & depression & -0.084 & 0.083 & -0.071 & -1.018 & 0.309 \\
\hline Disease duration & age & 0.126 & 0.103 & 0.080 & 1.218 & 0.233 \\
\hline Disease duration & MMSE & -0.025 & 0.017 & -0.086 & -1.517 & 0.129 \\
\hline Disease duration & $I A D L$ & -0.068 & 0.014 & -0.264 & -4.918 & $<0.001$ \\
\hline Disease duration & levodopa & 0.143 & 0.021 & 0.625 & 6.856 & $<0.001$ \\
\hline Disease duration & dopa-agonist & 0.030 & 0.012 & 0.164 & 2.480 & 0.013 \\
\hline Disease duration & UPDRS s.2 & 0.373 & 0.062 & 0.316 & 5.975 & $<0.001$ \\
\hline Disease duration & UPDRS s. 3 & 0.274 & 0.072 & 0.214 & 3.808 & $<0.001$ \\
\hline Disease duration & fluctuations & 0.004 & 0.024 & 0.018 & 0.175 & 0.861 \\
\hline Levodopa & fluctuations & 0.671 & 0.134 & 0.647 & 5.020 & $<0.001$ \\
\hline Disease duration & dyskinesias & -0.024 & 0.033 & -0.087 & -0.710 & 0.478 \\
\hline Levodopa & dyskinesias & 0.971 & 0.251 & 0.810 & 3.862 & $<0.001$ \\
\hline
\end{tabular}

$\mathrm{ADL}=$ Activities of Daily Living; $\mathrm{FH}$ = family history; IADL = Lawton's Instrumental Activities of Daily Living scale; MMSE = Mini Mental State Examination; s. = section; SE = standard error; UPDRS = Unified Parkinson's Disease Rating Scale.

In a first revision, paths that did not contribute substantially to the model were deleted: age, a family history of anxiety, the use of a dopamine agonist, the presence of dyskinesias, and the indirect effects of levodopa. This resulted in a second, simpler model showing similar fit (RMSEA 0.074 with a $90 \% \mathrm{Cl}=0.069-0.080 ; \mathrm{CFI}$ 0.675). This intermediate model is shown in Supplementary Figure 2, and specified in Supplementary Table 2. 
A second revision, which allowed for correlated residuals among variables and extra paths, resulted in a model with better fit (RMSEA 0.051 with a $90 \% \mathrm{Cl}=0.044-$ 0.058; CFI 0.854). In this last model, the disease-specific ADL parameter was removed, and an indirect effect of the MMSE on depression through IADL function was added, as well as a correction for interaction of IADL and UPDRS section 3. This model is shown in Figure 2 and specified in Table 3 . The model explains $41 \%$ of the observed variance in the depression outcome.

We finally compared the effects of the PD-specific and PD non-specific variables in the model after both types of variables had been regressed on two latent variables. This showed that both factors were significantly associated with depression and together accounted for $69 \%$ of its variance. The PD non-specific factor was significantly more strongly related to depression $(\beta=0.742, \mathrm{SE}=0.078, P$ $<0.001)$ than the PD-specific factor $(\beta=0.247, \mathrm{SE}=0.082, P=0.003)$. This was confirmed in a Wald test comparing the effects of the two factors $\left(\chi^{2}=12.93, \mathrm{df}=1\right.$, $P<0.001)$.

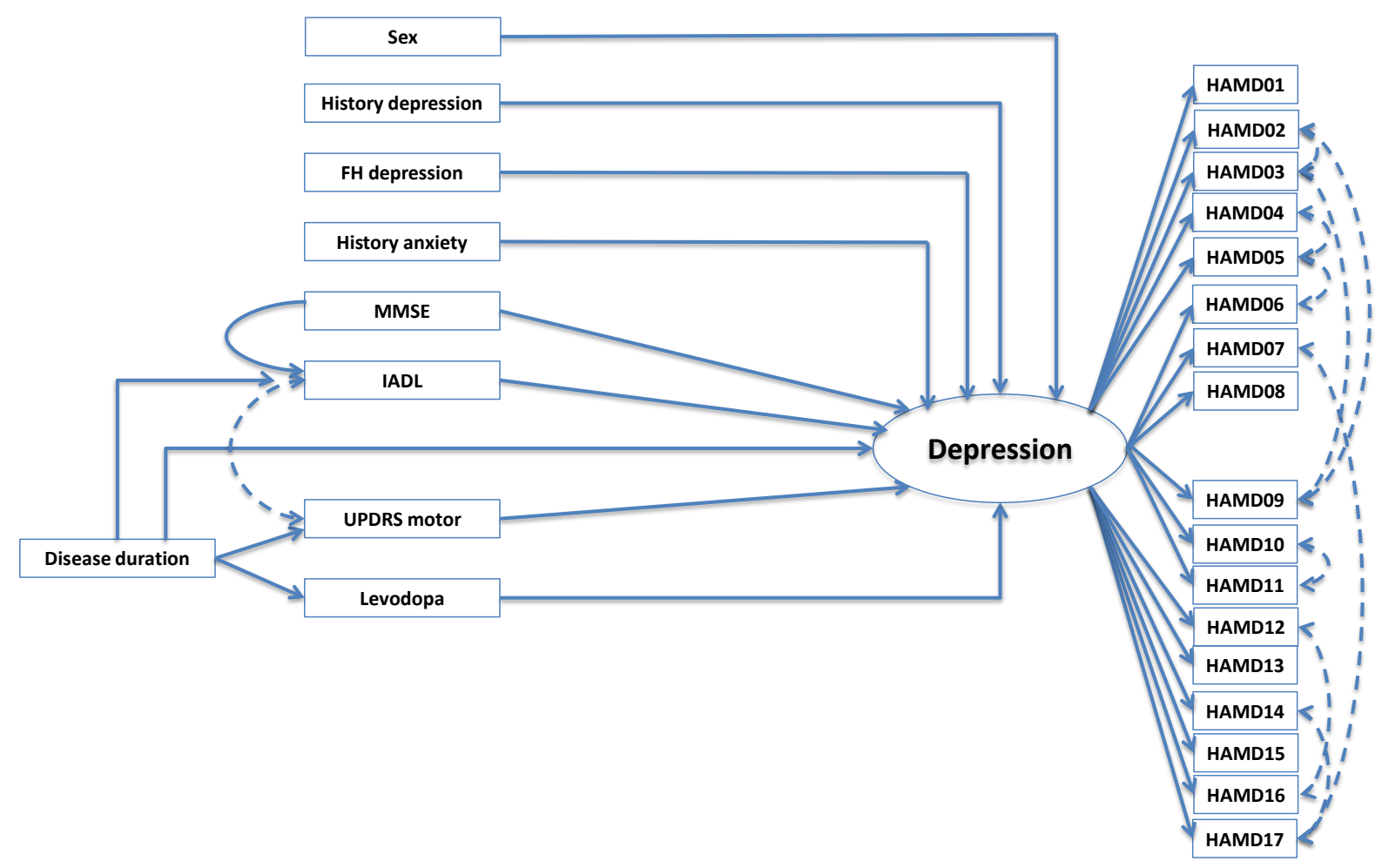

Figure 2. Final model of depression in PD (model 3). This final model allows for correlated residuals (dashed lines) among variables and extra paths. Model specifics and regression co-efficients are given in Table 3. FH = family history; HAMD = Hamilton Depression Scale; $I A D L=$ Lawton's Instrumental Activities of Daily Living scale; MMSE = Mini Mental State Examination; UPDRS = Unified Parkinson's Disease Rating Scale. 
Table 3. Standardized regression coefficients showing direct effects within specified paths for the final model including 8 variables (model 3 ).

\begin{tabular}{|c|c|c|c|c|c|c|}
\hline Parameter & $\begin{array}{l}\text { Dependent } \\
\text { variable }\end{array}$ & B & SE & $\beta$ & z-statistic & $P$-value \\
\hline Sex & depression & -0.442 & 0.163 & -0.166 & -2.711 & 0.007 \\
\hline $\begin{array}{l}\text { History } \\
\text { depression }\end{array}$ & depression & 0.670 & 0.179 & 0.256 & 3.743 & $<0.001$ \\
\hline History Anxiety & depression & 0.499 & 0.178 & 0.184 & 2.809 & 0.005 \\
\hline FH depression & depression & 0.312 & 0.147 & 0.140 & 2.116 & 0.034 \\
\hline MMSE & depression & -0.185 & 0.050 & -0.242 & -3.735 & $<0.001$ \\
\hline IADL & depression & -0.109 & 0.049 & -0.120 & -2.234 & 0.026 \\
\hline Disease duration & depression & -0.067 & 0.023 & -0.289 & -2.935 & 0.003 \\
\hline UPDRS motor (s.3) & depression & 0.056 & 0.012 & 0.302 & 4.722 & $<0.001$ \\
\hline Use of levodopa & depression & 0.306 & 0.109 & 0.303 & 2.799 & 0.005 \\
\hline Disease duration & UPDRS s.3 & 0.261 & 0.075 & 0.207 & 3.508 & $<0.001$ \\
\hline Disease duration & levodopa & 0.145 & 0.021 & 0.631 & 6.803 & $<0.001$ \\
\hline Disease duration & $I A D L$ & -0.065 & 0.014 & -0.253 & -4.753 & $<0.001$ \\
\hline MMSE & $I A D L$ & 0.171 & 0.037 & 0.202 & 4.557 & $<0.001$ \\
\hline
\end{tabular}

$\mathrm{FH}=$ family history; IADL = Lawton's Instrumental Activities of Daily Living scale; MMSE = Mini Mental State Examination; s. = section; SE = standard error; UPDRS = Unified Parkinson's Disease Rating Scale.

\section{Discussion}

In this study we showed that $41 \%$ of the variance in depressive symptoms in PD patients was explained by 9 variables: 6 variables not specific for PD, and 3 specific for PD. When both types of factors were regressed on two latent variables, they together explained $69 \%$ of variance. In this model, the latent variable representing the non-specific factors had a 3.0 times higher $\beta$, i.e., a three times larger influence, than the latent variable of all PD-specific factors. However, the manifest variables most strongly associated with depression in our final model were all PD specific markers: the UPDRS motor score, use of levodopa, and disease duration. Dopamine agonists have often been associated with mood-improving effects in PD patients. ${ }^{25}$ In our study however, the use of dopamine agonists does not have a significant negative (nor positive) association with the depression outcome. Taken together, both non-specific and PD-specific variables appear to contribute to depression in PD, with PD-specific factors showing strong individual associations, while non-specific factors seem to have a larger net effect on the depression outcome. 
Published research on the relative contribution of PD-specific and non-specific factors to the risk of depression in PD is scant. In one cross-sectional study involving 161 PD patients, a logistic regression model consisting of 5 general, PD-non-specific risk factors for depression (age, sex, prior history of depression, family history of depression, and somatic comorbidity), correctly predicted whether a patient was depressed or not in $75 \%$ of cases. Adding PD-specific risk factors into the logistic model did not increase the discriminative performance. ${ }^{26}$ Although other studies have included some general risk factors for depression, the influence of these factors has not been directly compared to that of PD-specific factors. In line with our findings, Riedel and colleagues as well as Becker and colleagues report female sex to be associated with depression. ${ }^{9,11}$ Higher age, cognitive decline, the use of non-steroid anti-inflammatory drugs (NSAIDs), and physical comorbidity other than PD are also related to depression in PD., ${ }^{8,11}$ Research seems to have moved away from the study of personality characteristics and coping in the etiology of depression in PD. Older studies report a premorbid personality in PD, characterized by inflexibility and obsessiveness, which may predispose for depression. ${ }^{27}$ Maladaptive cognitive coping is also associated with increased feelings of depression and anxiety. ${ }^{28,29}$ Finally, life events have been shown to play an important role in the development of major depression in PD, though its effect seems to be modified by social support and coping mechanisms. ${ }^{30}$ These factors should receive more consideration in future studies.

Our findings have important conceptual implications for our understanding of depression in PD. They show that several PD-specific factors are indeed important markers for depression, but that their true relevance is only understood by adopting a broad multifactorial approach to depression that also includes non-specific markers: PD specific factors are associated with depression, but their net effect is smaller than that of a number of general risk factors for depression, not specific for PD. Based on these findings one could hypothesize that those PD patients who develop depression are likely to have a pre-existing vulnerability due to their exposure to common risk factors for depression which are unrelated to PD. Our findings also imply that a broader approach should be followed in future studies into the etiology of depression in PD, including not only PD-specific, but also general non-specific risk factors.

Our study has several limitations. First of all, the analysis was performed on an existing database of a cross-sectional study into anxiety in patients with PD. Parameters selected to be included in the model were based on available data, 
which implies that other important parameters that may be considered markers of depression, such as marital status, the availability of a caregiver, personality, coping style, and past or recent life events were not included in the study. Also, protective factors, such as the use of antidepressants and recent positive life events were not included in the analysis. This may also explain why our final model explains $41 \%$ of the total variance in depression. Although this may seem a low percentage, it underscores that depressive syndromes are complex and etiologically multifactorial. Inclusion of more variables in the model would provide a more complete psychosomatic overview of all factors associated with depressive disturbances in PD. Notable psychological variables, such as the ones listed just above, warrant additional study given the fact that they are known risk factors for depression in the general population as well as in PD. Next, it is difficult to separate markers that are related to PD and those that are not directly related to PD. In PD patients, cognitive decline and decline in ADL functions may be due to PD, but these are also knownrisk factors for depression in the general population, and hence not specific for PD. This is why the authors used the terms 'PD-specific' and 'non-specific' rather than 'PD-related' and 'PD-unrelated' factors. Another limitation is the fact that our dataset is cross-sectional, and hence no causal interferences can be drawn. In spite of these shortcomings, the database was large enough to allow SEM analysis with a substantial number of parameters. Finally, model fit for all models was acceptable to good in terms of the RMSEA, although not in terms of CFI. Hence, our reliance on the RMSEA might seem arbitrary. However, CFI tends to worsen as the number of variables increases. ${ }^{31}$ In addition, use of the CFI (and other incremental measures of fit) has been discouraged if the baseline model has an RMSEA $<0.158,{ }^{32}$ which is the case in our data (RMSEA of the baseline model is 0.125). Again, these analyses are exploratory and limited as outlined above. Finally, the model requires confirmation in a longitudinal design that includes more psychological and contextual variables.

In this study, we showed that individual PD-specific factors are strongly associated with depression, but that non-specific factors, as compared to PD specific factors, contributed to a substantially larger degree to the presence of depressive disturbances. These data underscore the importance of understanding depression in PD within a complex multifactorial framework. Accordingly, it is critical that future studies into the etiology of depression in PD (and most likely studies addressing other psychopathological syndromes in PD as well) should have a wider scope and be designed to include general risk factors for depression, that are not specific for 
CHAPTER 6 | DEPRESSION IN PARKINSON'S DISEASE: MODELING RISKFACTORS

PD, as well as factors associated with PD. A restricted approach, limited to PDspecific factors, obscures the complex nature of psychopathological comorbidities encountered in PD, and may subsequently lead to wrong conclusions about what might be salient targets for prevention and treatment. 


\section{REFERENCES}

1. Reijnders JSAM, Ehrt U, Weber WEJ, Aarsland D, Leentjens AFG. A systematic review of prevalence studies of depression in Parkinson's disease. Movement Disorders 2008;23:183-189.

2. Global Parkinson's Disease Survey Steering Committee. Factors impacting on quality of life in Parkinson's disease: results from an international survey. Movement Disorders 2002;17:60-67.

3. Schrag A. Quality of life and depression in Parkinson's disease. Journal of the Neurological Sciences 2006;248:151-157.

4. Sonnenberg CM, Beekman ATF, Deeg DJH, Van Tilburg W. Sex differences in late life depression. Acta Psychiatrica Scandinavica 2000;101:286-292.

5. Von Korff MR, Scott KM, Gureje O. Global Perspectives on Mental-physical Comorbidity in the WHO World Mental Health Surveys. New York: Cambridge University Press, 2009.

6. Beekman ATF, Deeg DJH, Van Tilburg T, Smit JH, Hooijer C, Van Tilburg W. Major and minor depression in later life: a study of prevalence and risk factors. Journal of Affective Disorders 1995;36:65-75.

7. Schoevers RA, Beekman ATF, Deeg DJH, Geerlings MI, Jonker C, Van Tilburg W. Risk factors for depression in later life; results of a prospective community based study (AMSTEL). Journal of Affective Disorders 2000;59:127-137.

8. Dissanayaka NNW, Sellbach A, Silburn PA, O'Sullivan JD, Marsh R, Mellick GD. Factors associated with depression in Parkinson's disease. Journal of Affective Disorders 2011;132:82-88.

9. Becker C, Brobert GP, Johansson S, Jick SS, Meier CR. Risk of incident deporession in patients with Parkinson disease in the UK. European Journal of Neurology 2011;18:448-453.

10. Gallagher DA, Schrag A. Psychosis, apathy, depression and anxiety in Parkinson's disease. Neurobiology of Disease 2012;46:581-589.

11. Riedel O, Heuser I, Klotsche J, Dodel R, Wittchen HU, GEPAD Study Group. Occurrence risk and structure of depression in Parkinson disease with and without dementia: results from the GEPAD Study. Journal of Geriatric Psychiatry and Neurology 2010;23:27-34.

12. Leentjens AFG, Dujardin K, Marsh L, Richard IH, Starkstein SE, Martinez-Martin P. Anxiety rating scales in Parkinson's disease: a validation study of the Hamilton Anxiety Rating Scale, The Beck Anxiety Inventory and the Hospital Anxiety and Depression Scale. Movement Disorders 2011;26:407-415.

13. Leentjens AFG, Dujardin K, Marsh L, Richard IH, Starkstein SE, Martinez-Martin P. Symptomatology and markers of anxiety disorders in Parkinson's disease: a crosssectional study. Movement Disorders 2011;26:484-492.

14. De Rijk MC, Rocca WA, Anderson DW, Melcon MO, Breteler MMB, Maraganore DM. A population perspective on diagnostic criteria for Parkinson's disease. Neurology 1997;48:1277-1281.

15. Folstein MF, Folstein SE, McHugh PR. Mini Mental State. A practical method for grading the cognitive state of patients for the clinician. Journal of Psychiatric Research 1975;12:189-198. 
16. Dubois B, Burn D, Goetz C, et al. Diagnostic procedures for Parkinson's disease dementia: recommendations from the Movement Disorder Society task force. Movement Disorders 2007;16:2314-2324.

17. Fahn S, Elton RL, and members of the UPDRS committee. Unified Parkinson's Disease Rating Scale. In: Fahn S, Marsden CD, Goldstein M, Calne DB, eds. Recent developments in Parkinson's Disease. New Jersey: McMillan Health Care, 1987: 153163.

18. Hoehn MM, Yahr MD. Parkinsonism: onset, progression and mortality. Neurology 1967; 17:427-442.

19. Lawton MP, Brody EM. Assessment of older people: Self-maintaining and instrumental activities of daily living. Gerontologist 1969;9:179-186.

20. Hamilton M. A rating scale for depression. Journal of Neurology, Neurosurgery and Psychiatry 1960;23:56-62.

21. Hamilton M. The assessment of anxiety states by rating. British Journal of Medical Psychology 1959;32:50-55.

22. Schrag A, Barone $P$, Brown RG, et al. Depression rating scales in Parkinson's disease: critique and recommendations. Movement Disorders 2007;22:1077-1092.

23. Sheehan DV, Lecrubier $\mathrm{Y}$, Sheehan $\mathrm{KH}$, et al. The Mini-International Neuropsychiatric Interview (M.I.N.I.): the development and validation of a structured diagnostic psychiatric interview for DSM-IV and ICD-10. Journal of Clinical Psychiatry 1998;59 Suppl 20:22-33.

24. Byrne BM. Structural Equation Modeling with Mplus: Basic Concepts, Applications, and Programming. . New York: Taylor \& Francis Group, 2011.

25. Leentjens AFG. The role of dopamine agonists in the treatment of depression in patients with Parkinson's disease: a systematic review. . Drugs 2011;71:1-14.

26. Leentjens AFG, Lousberg R, Verhey FRJ. Markers for depression in Parkinson's disease. Acta Psychiatrica Scandinavica 2002;106:196-201.

27. Todes CJ, Lees A. The pre-morbid personality of patients with Parkinson's disease. Journal of Neurology Neurosurgery and Psychiatry 1985;48:97-100.

28. Allott R, Wells A, Morrison AP, Walker R. Distress in Parkinson's disease: contributions of disease factors and metacognitive style. British Journal of Psychiatry 2005;187:182183.

29. MacCarthy B, Brown R. Psychosocial factors in Parkinson's disease. British Journal of Clinical Psychology 1989;28:41-52.

30. Rod NH, Bordelon Y, Thompson A, Marcotte E, Ritz B. Major life events and the development of major depression in Parkinson disease patients. European Journal of Neurology 2012;doi: 10.1111/ene.12019. [Epub ahead of print]..

31. Kenny DA, McCoach DB. Effect of the number of variables on measures of fit in structural equation modelling. Structural Equation Modelling: a multidisciplinary journal 2003;10:333-351.

32. Kenny DA. http://davidakenny.net/cm/fit.htm [online]. Available at: http://davidakenny.net/cm/fit.htm. Accessed 27 January 2013. 
Supplementary Table 1. Correlation matrix of variables included in the initial model (model 1).

\begin{tabular}{|l|l|l|l|l|l|l|}
\hline & $\begin{array}{l}\text { UPDRS } \\
\text { s.3 }\end{array}$ & $\begin{array}{l}\text { UPDRS } \\
\text { s.4 }\end{array}$ & H\&Y & off-periods & dyskinesias & IADL \\
\hline UPDRS s.2 & $\mathbf{0 . 5 1}$ & $\mathbf{0 . 4 9}$ & $\mathbf{0 . 6 0}$ & & & 0.46 \\
\hline UPDRS s.3 & & & $\mathbf{0 . 4 6}$ & & & \\
\hline UPDRS s.4 & & & & 0.66 & & \\
\hline H\&Y & & & & & & $\mathbf{0 . 5 1}$ \\
\hline off-periods & & & & & 0.43 & \\
\hline dyskinesias & & & & & & \\
\hline IADL & & & & & & \\
\hline
\end{tabular}

Only parameters with significant correlation coefficients larger than 0.4 are shown. Significance is defined here as $P<0.005$ (after correction for multiple testing). Variables not shown (sex, age, the use of a dopamine agonist or levodopa) have either no significant $(P \geq 0.005)$ or only weak $(P \leq 0.4)$ correlations with any of the other variables.

UPDRS = Unified Parkinson's Disease Rating Scale; s. = section; H\&Y = Hoehn and Yahr staging system; IADL = Lawton's Instrumental Activities of Daily Living scale.

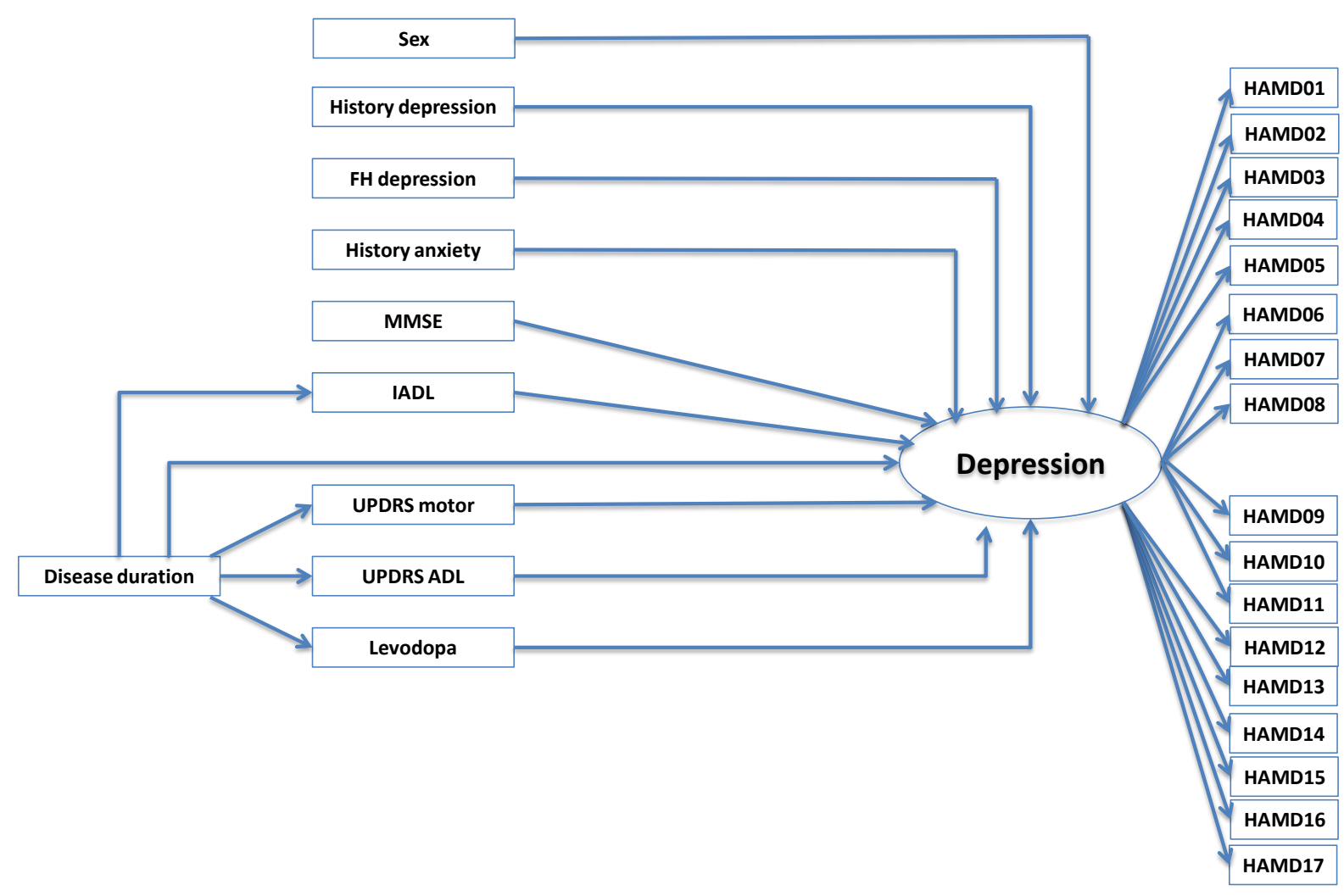

Supplementary Figure 1. Adjusted model of depression in PD (model 2). In this model, all paths that did not contribute substantially to the initial model were deleted. Model specifics and regression co-efficients are given in Supplementary Table 2. ADL = Activities of Daily Living; FH = family history; HAMD = Hamilton Depression Scale; IADL = Lawton's Instrumental Activities of Daily Living scale; MMSE = Mini Mental State Examination; UPDRS = Unified Parkinson's Disease Rating Scale. 
CHAPTER 6 | DEPRESSION IN PARKINSON'S DISEASE: MODELING RISKFACTORS

Supplementary Table 2. Standardized regression coefficients of the intermediate model including 10 variables (model 2)

\begin{tabular}{llrrrrr}
\hline Parameter & $\begin{array}{l}\text { Dependent } \\
\text { variable }\end{array}$ & B & SE & $\boldsymbol{\beta}$ & z-statistic & $\boldsymbol{P}$-value \\
\hline Sex & depression & -0.389 & 0.146 & -0.162 & -2.725 & 0.006 \\
History depression & depression & 0.606 & 0.160 & 0.251 & 3.800 & $<0.001$ \\
History Anxiety & depression & 0.462 & 0.159 & 0.185 & 2.900 & 0.004 \\
FH depression & depression & 0.281 & 0.133 & 0.136 & 2.118 & 0.034 \\
MMSE & depression & -0.184 & 0.046 & -0.260 & -3.981 & $<0.001$ \\
IADL & depression & -0.191 & 0.048 & -0.223 & -3.997 & $<0.001$ \\
Disease duration & depression & -0.081 & 0.023 & -0.378 & -3.590 & $<0.001$ \\
UPDRS motor (s.3) & depression & 0.057 & 0.011 & 0.334 & 5.390 & $<0.001$ \\
UPDRS ADL (s. 2) & depression & 0.054 & 0.011 & 0.296 & 4.845 & $<0.001$ \\
Use of levodopa & depression & 0.229 & 0.100 & 0.245 & 2.290 & 0.022 \\
& & & & & & \\
Disease duration & IADL & -0.065 & 0.014 & -0.259 & -4.753 & $<0.001$ \\
Disease duration & UPDRS s.2 & 0.359 & 0.062 & 0.307 & 5.753 & $<0.001$ \\
Disease duration & UPDRS s.3 & 0.261 & 0.075 & 0.207 & 3.508 & $<0.001$ \\
Disease duration & levodopa & 0.145 & 0.021 & 0.631 & 6.803 & $<0.001$ \\
\hline ADL Activis of Dally
\end{tabular}

$\mathrm{ADL}=$ Activities of Daily Living; $\mathrm{FH}=$ family history; IADL = Lawton's Instrumental Activities of Daily Living scale; MMSE = Mini Mental State Examination; $\mathrm{s}$. = section; SE = standard error; UPDRS = Unified Parkinson's Disease Rating Scale. 


\section{CHAPTER 7}

\section{SEVERITY OF DEPRESSION AND ANXIETY ARE PREDICTORS OF RESPONSE TO}

\section{ANTIDEPRESSANT tReatment in PARKINSON's disease}

Parkinsonism and Related Disorders, 20: 644-646, 2014

Anja J.H. Moonen*, Anke Wijers*, Albert F.G. Leentjens*, C.W. Christine, S. Factor, J.L. Juncos, J. Lyness, Laura Marsh, M. Panisset, R.P. Pfeiffer, D.A. Rottenberg, C. Serrano Ramos, L. Shulman, C. Singer, J.T. Slevin, W.M. McDonald, P. Auinger, Irene H. Richard

* These authors are dual first authors 


\section{ABSTRACT}

Background: Antidepressants have appeared to be more effective than placebo treatment in treating depressive syndromes in patients with Parkinson's disease (PD). Objective: To identify factors that predict improvement in depressive symptoms during antidepressant treatment in depressed PD patients.

Methods: A secondary analysis was performed on the dataset of the Randomized Placebo-controlled Study of Antidepressants in PD (SAD-PD), in which 76 patients received active treatment with either paroxetine or venlafaxine extended release $(X R)$, and 39 patients received placebo treatment. Backward stepwise regression analyses were conducted with change in 24-item Hamilton Depression Rating Scale (HAMD24) score between assessments at baseline and week 12 as the main outcome measure, and sex, age, baseline HAMD-24 score, Unified Parkinson's Disease Rating Scale section III (UPDRS-III) score, Mini-Mental State Examination (MMSE), and the Clinical Anxiety Scale (CAS) as independent variables.

Results: In both the active treatment and placebo groups, higher baseline HAMD-24 score and lower UPDRS-III score were associated with greater reduction in HAMD24 score. Higher anxiety scores predicted less response in the active treatment group. Higher MMSE scores predicted greater response only in the placebo treated group. Sex and age were no predictors of response.

Conclusions: Higher pre-treatment depression scores and lower pre-treatment anxiety scores are the two most important predictors for improvement during antidepressant treatment in depressed PD patients, which is in line with those found in treatment studies of depressed non-PD patients. Furthermore, our results indicate the requirement for different or more intensive treatment for depressed PD patients with more severe anxiety symptoms. 


\section{INTRODUCTION}

Depressive syndromes frequently occur in Parkinson's disease (PD), affecting quality of life, activities of daily living (ADL), motor function and cognitive function..$^{1-4}$ On average, $17 \%$ of PD patients suffer from a major depressive disorder, $22 \%$ from minor depression and $13 \%$ from dysthymia. ${ }^{1}$ Thirty-five percent of PD patients suffer from clinically significant depressive symptoms, irrespective of a DSM diagnosis of depressive disorder. ${ }^{1}$ Of those patients diagnosed with depression, only $35 \%$ received antidepressant treatment, and in only half of these was the treatment adequate. ${ }^{5}$ Recently, the randomized controlled Study of Antidepressants in PD (SAD-PD) demonstrated the effectiveness of paroxetine (a selective serotonin reuptake inhibitor) and venlafaxine extended release (XR) (a selective serotonin and norepinephrine reuptake inhibitor) in the active treatment of depressive syndromes in PD patients as compared to a placebo treatment. For both the paroxetine group and venlafaxine XR group the reduction on the 24-item Hamilton Depression Scale (HAMD-24) after 12 weeks (13 and 11 points respectively) was larger than that in the placebo group (6.8 points). ${ }^{2}$ Moreover, self-ratings at week 12 showed that $56 \%$ of the patients who received treatment with paroxetine and $43 \%$ of those who received venlafaxine $X R$ reported substantial improvement of depressive symptoms. An important next question would be whether there are factors that predict the efficacy of antidepressant treatment. $^{2}$

This paper presents a secondary analysis of the SAD-PD study data. Its aim was to identify factors that predict improvement in depressive symptoms during antidepressant treatment in depressed PD patients.

\section{METHODS}

This study is a secondary analysis on the dataset of the SAD-PD study. For a detailed description of the methods, the authors refer to Richard and colleagues. ${ }^{2}$ Here, we summarize the design and methods of the original study.

\section{SAD-PD study design:}

The SAD-PD study was a three arm randomized placebo-controlled clinical trial of the efficacy of the treatment of depression in PD patients (trial registration no NCT00086190). 115 PD-patients from 20 movement disorder clinics, suffering from either major depression, minor depression or dysthymia, as diagnosed with the Structured Clinical Interview for DSM-IV (SCID), or from 'operationally defined 
subsyndromal depression' (2; supplemental data), were randomized to treatment with paroxetine $(n=42)$, venlafaxine $X R(n=34)$ or placebo $(n=39)$. For a detailed participant flow chart we refer to Richard and colleagues. ${ }^{2}$

The double-blind treatment phase lasted 12 weeks. Patient visits took place at baseline and after 2, 4, 6, 8 and 12 weeks. Maximum daily dosages were 40mg for paroxetine and 225mg for venlafaxine XR. PD medications were optimized prior to participation and dosages were kept stable during the study as much as possible. The primary outcome measure was change in the HAMD-24 from baseline to week 12. In addition, motor function was quantified with the motor section of the Unified Parkinson's Disease Rating Scale (UPDRS-III), global cognitive function was assessed with the Mini Mental State Examination (MMSE), and anxiety was assessed with the Clinical Anxiety Scale (CAS). The latter scale is a 6-item rating scale, derived from the Hamilton Anxiety Rating Scale. All assessments took place while subjects were in their "on" state.

Approvals, registrations, and patient consents:

The study was approved by the local Medical Ethics Committees of all participating institutions. All patients gave written informed consent.

Materials and statistical methods of the current study:

All of the 115 patients from the SAD-PD study who completed the study were included in the current secondary analysis. Demographic and clinical variables of both active treatment groups taken together and the placebo group were compared with the chi-square test for categorical variables and the Kruskal-Wallis test for continuous variables. The active treatment groups were considered as one single group to achieve a higher group size, which allows for more independent variables in the regression analyses. In order to identify predictors of improvement in depressive symptoms, backward stepwise regression analyses were performed for the active treatment and placebo group. Change in HAMD-24 score between baseline and follow-up at 12 weeks was the primary outcome measure. Independent variables were sex, age, baseline HAMD-24 score, UPDRS-III, MMSE and CAS scores. Prior to analysis, we confirmed that the data met the assumptions of regression analyses: normality, homogeneity of variance and non-collinearity. The level of significance was set at 0.05 . All analyses were performed with IBM SPSS 21.0 software. 


\section{RESULTS}

Of the 115 randomized patients, 97 completed the study: 34 in the paroxetine group, 30 in the venlafaxine XR group and 33 in the placebo group. Baseline characteristics of patients included in the analysis are shown in Table 1. There were no significant differences between the two treatment groups for any of the baseline characteristics.

Table 1. Baseline Characteristics of included patients in the active and placebo treated groups.

\begin{tabular}{|c|c|c|c|c|c|}
\hline & & \multicolumn{2}{|c|}{ Active Treatment $(n=64)$} & \multicolumn{2}{|c|}{ Placebo $(n=33)$} \\
\hline \multicolumn{2}{|l|}{ Age } & 63.6 & $(10.3)$ & 61.9 & $(10.0)$ \\
\hline \multicolumn{2}{|c|}{ Sex $(\%$ male $)$} & \multicolumn{2}{|c|}{62.5} & \multicolumn{2}{|c|}{58.8} \\
\hline \multicolumn{2}{|c|}{ Years Since PD Onset } & 7.2 & $(4.3)$ & 7.3 & (3.8) \\
\hline \multicolumn{2}{|c|}{ Years Since PD Diagnosis } & 5.1 & $(4.1)$ & 5.4 & (3.9) \\
\hline \multirow[t]{3}{*}{ H\&Y Stage $(\%)$} & $1.0-1.5$ & \multicolumn{2}{|c|}{3.1} & \multicolumn{2}{|c|}{5.9} \\
\hline & $2.0-2.5$ & \multicolumn{2}{|c|}{81.3} & \multicolumn{2}{|c|}{79.4} \\
\hline & $3.0-4.0$ & \multicolumn{2}{|c|}{15.6} & \multicolumn{2}{|c|}{14.7} \\
\hline \multirow[t]{4}{*}{ UPDRS } & I (Mental) & 4.9 & $(2.2)$ & 4.7 & $(2.0)$ \\
\hline & II (ADL) & 10.7 & $(7.0)$ & 10.9 & $(5.3)$ \\
\hline & III (Motor) & 27.0 & $(10.9)$ & 25.9 & $(11.7)$ \\
\hline & Total & 42.4 & (17.1) & 41.4 & $(16.0)$ \\
\hline Use of PD & Levodopa & \multicolumn{2}{|c|}{82.8} & \multicolumn{2}{|c|}{79.4} \\
\hline \multirow{4}{*}{ medication (\%) } & Dopa- Agonist & \multicolumn{2}{|c|}{54.7} & \multicolumn{2}{|c|}{52.9} \\
\hline & COMT Inhibitor & \multicolumn{2}{|c|}{18.8} & \multicolumn{2}{|c|}{32.4} \\
\hline & Amantadine & \multicolumn{2}{|c|}{12.5} & \multicolumn{2}{|c|}{17.6} \\
\hline & Anticholinergic & \multicolumn{2}{|c|}{10.9} & \multicolumn{2}{|c|}{8.8} \\
\hline \multicolumn{2}{|c|}{ Baseline HAMD-24 } & 21.8 & $(6.2)$ & 21.4 & $(4.8)$ \\
\hline \multicolumn{2}{|c|}{ CAS } & 7.4 & $(4.2)$ & 7.2 & (3.8) \\
\hline \multicolumn{2}{|l|}{ MMSE } & 29.0 & $(1.5)$ & 28.5 & $(1.5)$ \\
\hline \multicolumn{2}{|c|}{ PDQ-39 } & 36.6 & $(16.3)$ & 39.7 & $(15.6)$ \\
\hline Major Depressic & $(\%)$ & \multicolumn{2}{|c|}{65.6} & \multicolumn{2}{|c|}{58.8} \\
\hline
\end{tabular}

Values are presented as mean (SD) unless otherwise indicated. $A D L=$ Activities of daily living; $C A S=$ Clinical Anxiety Scale; COMT = Catechol-O-methyltransferase; HAMD = Hamilton Depression Rating Scale; $\mathrm{H} \& Y=$ Hoehn and $Y$ ahr staging system; MMSE = Mini Mental State Examination; UPDRS = Unified Parkinson's Disease Rating Scale.

Backwards stepwise regression analyses were performed in order to identify predictors of change in HAMD-24 score for the active treatment group and placebo group. In the active treatment group, three predictors remained in the regression model after conducting the analyses. Higher pre-treatment HAMD-24 score, lower baseline anxiety score (CAS), and a lower UPDRS-III score were significant predictors of improvement in HAMD-24 score. Of these, pre-treatment HAMD-24 score was the strongest predictor, with an even three times greater influence in the 
regression model than the other two predictors (Table 2). The model explained $43 \%$ of the variance in the improvement in HAMD-24 score.

In the placebo group, backward stepwise regression analyses showed that a higher HAMD-24 score, and lower UPDRS-III score, both at baseline, were associated with greater reduction in HAMD-24 (Table 2). A higher MMSE score was marginally significant (Table 2). These three variables had a comparable influence in the model, which overall explained $26 \%$ of the variance in the change on the HAMD24 score. For both the active treatment group and placebo group, age and sex were no significant predictors of change in HAMD-24 score.

Table 2: Backward-stepwise regression analysis for predictors of greater reduction in HAMD24 score in the active treatment group versus placebo group.

\begin{tabular}{lcccccc} 
Group & B & {$[95 \%$ CI } & SE & Beta & T-value & $P$-value \\
\hline $\begin{array}{l}\text { ACTIVE TREATMENT } \\
\text { Constant }\end{array}$ & 0.034 & {$[-4.841-4.910]$} & 2.437 & & 0.014 & 0.989 \\
HAMD-24 Baseline & 0.705 & {$[0.494-0.916]$} & 0.105 & .741 & 6.694 & $<0.001$ \\
CAS & -0.381 & {$[-0.689--0.072]$} & 0.154 & -.272 & -2.468 & 0.016 \\
UPDRS III & -0.111 & {$[-0.219--0.003]$} & 0.054 & -.251 & -2.060 & 0.044 \\
PLACEBO & & & & & & \\
Constant & -46.521 & {$[-98.784-5.742]$} & 25.591 & & -1.818 & 0.079 \\
HAMD-24 Baseline & 0.595 & {$[0.043-1.1469]$} & 0.270 & .412 & 2.203 & 0.035 \\
MMSE & 1.647 & {$[-0.006-3.300]$} & 0.809 & .346 & 2.034 & 0.051 \\
UPDRS III & -0.263 & {$[-0.483--0.043]$} & 0.108 & -.429 & -2.444 & 0.021 \\
\hline ACtive
\end{tabular}

Active treatment: Model fit: R2 = .434; Excluded Variables: Age, Sex, MMSE score. Placebo:

Model fit: R2 = .259; Excluded Variables: Age, Sex, CAS score. SE = standard error.

\section{Discussion}

The present study aimed to identify predictors of improvement in depressive symptoms in PD patients who received either anti-depressant treatment (i.e., paroxetine or venlafaxine XR) or placebo treatment. For the active treatment group, a higher baseline HAMD-24 score was found to be the most important predictor of improvement in depressive symptoms after 12 weeks, with lower baseline anxiety score being the second most important predictor. Both these variables have been 
identified as predictors of antidepressant response in the treatment of depressed patients without PD., ${ }^{3,6,7}$ These findings imply that depressed PD patients with more severe anxiety may need more intensive or different treatments.

In the placebo group, a higher baseline HAMD-24 score was also associated with a greater reduction in HAMD-24 score. This is surprising since in most studies involving non-PD subjects, a higher baseline HAMD-24 score is associated with a smaller placebo response.$^{3,8} \mathrm{~A}$ possible explanation for this may be the fact that this study included patients with less severe depressive syndromes. This may lead to lower HAMD scores compared to those found in other studies in which only patients who suffered from major depression were selected. This in turn would have limited the placebo response. Interestingly, in the placebo group a higher MMSE and lower UPDRS-III score predict a larger reduction in HAMD-24 score. The placebo response is known to be related to expectation of reward (or in this case: improvement of depressive symptoms), which in turn depends on information processing skills. Therefore a better cognitive status may help to enhance these expectations, and potentially induce a larger placebo response. In addition, better cognitive functioning and less motor problems may indicate less severe injury of the dopaminergic pathways, including those in the mesolimbic reward system, which may be involved in the placebo response. ${ }^{9}$

This study has several limitations. First, with an $R^{2}$ of $43 \%$ for the active treatment group and $26 \%$ for the placebo group, a large part of the variance remains unexplained. This implies that, additional variables, not included in this analysis, may be responsible for the unaccounted variance. ${ }^{3,6,8,10}$ Alternatively, if the sample size per group had been bigger, perhaps other (non-significant) predictors may have added value to the model. Secondly, although the ratio of number of predictors to number of subjects was adequate for the active treatment group, it may have been high for the placebo group. This could increase the chance of a type 2 error. Finally, in order to increase the number of independent variables that could be included in the regression analysis, both active treatment groups were considered as one. Subanalyses of data in the original paper suggest that venlafaxine $X R$ may be more effective than paroxetine in women, in patients who use dopamine agonists, and in patients with longer disease duration [Ref ${ }^{2}$; supplemental data]. However, in posthoc per group regression analyses we did not identify different predictors in the venlafaxine $X R$ group. In the paroxetine group, pre-treatment depression score remained as the only significant predictor of improvement of depressive symptoms. 
Moreover, due to power issues, we were not able to differentiate between the influence of different dopaminergic medications on both treatment groups separately.

In conclusion, in this study we found that the two most important predictors of response during antidepressant treatment of depressed PD patients were higher pretreatment depression scores and lower pre-treatment anxiety scores. These predictors are consistent with those found in treatment of depressed non-PD patients, which seems to indicate that these predictors are not specific for PD. Our findings further imply that depressed PD patients with more severe anxiety may need more intensive or different treatment. 


\section{REFERENCES}

1. Reijnders JSAM, Ehrt U, Weber WEJ, Aarsland D, Leentjens AFG. A Systematic Review of Prevalence Studies of Depression in Parkinson's Disease. Mov Disord. 2008;23(2):183-9.

2. Richard IH, McDermott M, Kurlan R, Lyness J, Como $P$, Pearson $\mathrm{N}$, et al. A randomized, double-blind, placebo-controlled trial of antidepressants in Parkinson disease. Neurology. 2012;78(16):1129-36.

3. Khan A, Leventhal RM, Khan SR, Brown WA. Severity of Depression and Response to Antidepressants and Placebo: An Analysis of the Food and Drug Administration Database. J Clin Psychopharmacol. 2001;22(1):40-5.

4. Santangelo G, Vitale C, Trojano L, et al. Relationship between depression and cognitive dysfunction in Parkinson's disease without dementia. Journal of Neurol 2009;256:632-638.

5. Weintraub, D., Moberg, P. J., Duda, J. E., Katz, I. R., \& Stern, M. B. (2003). Recognition and treatment of depression in Parkinson's disease. J Geriatr Psych Neur, 16(3), 178-183.

6. Ising M, Lucae S, Binder E, Bettecken T, Uhr M, Ripke S, et al. A Genomewide Association Study Points to Multiple Loci That Predict Antidepressant Drug Treatment Outcome in Depression. Arch Gen Psychiatry. 2009;66(9):966-75.

7. Cohen A, Gilman SE, Houck PR, Szanto K, Reynolds CF 3rd. Socioeconomic status and anxiety as predictors of antidepressant treatment response and suicidal ideation in older adults. Soc Psychiatry Psychiatr Epidemiol. 2009;44:272-7.

8. Nelson JC, Zhang Q, Deberdt W, Marangell LB, Karamustafalioglu O, Lipkovich IA. Predictors of remission with placebo using an integrated study database from patients with major depressive disorder. Curr Med Res Opin. 2012;28:325-34.

9. Tobler PN, Fiorillo CD, Schultz W. Adaptive coding of reward value by dopamine neurons. Science 2005;307 (5715) 1642- 1645.

10. Hennings JM, Owashi T, Binder EB, Horstmann S, Menke A, Kloiber S, et al. Clinical characteristics and treatment outcome in a representative sample of depressed inpatients - Findings from the Munich Antidepressant Response Signature (MARS) project. J Psychiatr Res. 2008;43:215-29. 



\section{ChAPTER 8}

General Discussion 
The main aim of this thesis was to gain a better understanding of the underlying neurobiological mechanisms of neuropsychiatric disorders in Parkinson's disease (PD) by using various methods of magnetic resonance imaging (MRI). More specifically, we used functional and structural MRI techniques to study respectively the neural substrates of emotional processing (Chapter 3 ) and cognitive disorders (Chapter 5) in PD. In addition, we aimed to study risk factors that contribute to the development of depression in PD, and to successful antidepressant treatment. This chapter discusses the interpretation of our main findings, as well as the scientific and clinical implications. In addition, some important methodological issues will be addressed. To conclude, recommendations for future research are given.

\section{MAIN FINDINGS}

\section{Emotional processing}

In Chapter 2 we performed an extensive review on the evidence base of changes in emotional processing and its neurobiological correlates in patients with PD. The included studies have investigated multiple aspects of emotional processing, such as facial emotion recognition, physiological arousal, and emotional prosody. There was a large variability in methodology and clinical profiles among studies, making it difficult to draw unambiguous conclusions about the nature of emotional processing deficits in PD. However, the majority of studies reported that PD patients showed an intact ability to explicitly recognize and evaluate emotional stimuli. Despite this, several studies using structural MRI, functional MRI or electroencephalogram (EEG), found reduced grey matter density and altered brain activity in regions involved in implicit emotional processing, including the amygdala, anterior cingulate cortex (ACC), striatum, and prefrontal cortices.

According to neurobiological models, implicit and explicit aspects of emotional processing are associated with the involvement of respectively two separate neural systems: a ventral and dorsal system. ${ }^{1-3}$ Here, specific parts of a predominantly ventral system are critical for the implicit perception of emotions and for generating an appropriate behavioral emotional response. Regions within this "emotional" circuit mainly include frontal-subcortical limbic areas (e.g., amygdala, ventral striatum, ventral ACC, orbitofrontal cortex) that are highly dependent on dopaminergic neurotransmission, and have been described to be affected in PD. Regions within the dorsal system, on the other hand, (e.g., dorsolateral and dorsomedial prefrontal 
cortex, dorsal ACC, hippocampus) are more involved in top-down regulation of emotional appraisal and the affective state. This requires cognitive and regulatory aspects of emotion, which seem to remain largely intact in PD patients. The fact that PD patients are able to cognitively regulate or modulate their emotional responses despite reduced dopamine supplies, raised the question whether compensational neural mechanisms or behavioral strategies are effective in PD.

In Chapter 3, we aimed to address this question by investigating implicit emotional processing in medicated PD patients and healthy control subjects using blood-oxygen level dependent fMRI (BOLD-fMRI). We found reduced striatal activity but increased medial prefrontal activation and an intact ability in PD patients to explicitly recognize and evaluate emotional stimuli. An important role for the medial prefrontal network in emotion regulation has been described in the literature. ${ }^{2,4,5}$ The increased medial prefrontal activation in PD patients may have served as a compensatory (top-down) cognitive control mechanism to either restore or compensate for the disturbed subcortical limbic activity, or to modulate their emotional response to intense emotional stimuli by using emotion regulation strategies. Future neuroimaging studies should further explore the role of the medial prefrontal network in emotion regulation in PD, by measuring functional connectivity patterns between frontal and subcortical structures during emotional processing.

\section{Cognitive disorders}

Cognitive deficits are common in PD and already occur at early stages of the disease. The clinical presentation and progression of cognitive decline in PD has appeared to be heterogeneous and several attempts have been made to identify distinctive clinical profiles. ${ }^{6}$ Instead of using predefined categories, such as PD patients with mild cognitive impairment (PD-MCl) or with PD dementia (PDD), Chapter 4 describes a data-driven approach in which patient subgroups were based on cognitive performance at a large battery of neuropsychological tests. In this prospective cross-sectional study, we validated five cognitive profiles that were previously identified by an exploratory cluster analysis on retrospective neuropsychological data. ${ }^{7}$ As such, we identified the following five cognitive phenotypes: 1) Cognitively intact patients with normal performance in all cognitive domains $(25.64 \%), 2)$ Cognitively intact patients with slight mental slowing $(26.92 \%)$, 3) Patients showing mild cognitive deficits in attention, working memory, executive functioning, language and visuospatial functioning and slight deficits in episodic 
verbal memory with intact recognition $(37.18 \%), 4)$ Patients with impaired overall cognitive efficiency and severe deficits in all cognitive domains, particularly executive functioning (3.2\%), and 5) Patients with impaired overall cognitive efficiency and severe deficits in all cognitive domains, particularly working memory and recall in verbal episodic memory (7.05\%).

In Chapter 5, we aimed to identify regional differences in grey matter (GM) atrophy between each of the cognitive phenotypes in a prospective cohort study using structural MRI. Optimized voxel-based morphometry (VBM-Dartel) revealed a pattern of cerebral atrophy that could already be detected in PD patients with intact cognition. These patients showed a similar pattern of GM atrophy that is characteristic for PD patients with dementia, including the parietal-temporal lobe, prefrontal cortex, and posterior cingulate. ${ }^{8-10}$ The only difference between their cognitive profile and that of cognitively intact patients was related to slowed mental speed. These results provide important support for the view that slowed mental speed and it's corresponding pattern of grey matter loss can be considered as an early marker of cognitive decline in PD. Patients with a profile close to dysexecutive $\mathrm{MCl}$ showed increased GM atrophy in the parietal-temporal lobe, posterior cingulate, and bilateral amygdala. In addition, we observed GM loss in the bilateral hippocampus, therewith supporting the view that hippocampal neurodegeneration can be associated with early cognitive decline in PD-MCl. ${ }^{10}$ Patients with global cognitive decline and a profile of severe cognitive deficits in multiple domains showed a similar pattern of GM atrophy, yet additional GM loss in the left medial temporal lobe and left parahippocampal gyrus. This extension to medial temporal areas is in line with other VBM studies, ${ }^{8,10}$ and possibly reflects more widespread Lewy body pathology as well as a risk factor for conversion to PD dementia (PDD). Alternatively, the observed GM abnormalities may reflect an interaction of different neurodegenerative processes such as comorbid Alzheimer's disease pathology. ${ }^{11}$

Important to note is the substantial influence of age when investigating neuroanatomical correlates of cognitive disorders in PD. In our study, age appeared to be the main predictor of cognitive decline in patients groups with slowed mental speed and PD-MCl. Age was an even more important predictor of the rate of cognitive decline than age at onset of PD or disease duration. We only observed potential disease-related atrophy in addition to effects of advanced aging in the cognitively worst group. In order to disentangle the absolute contribution of disease 
to cognitive decline independently of age, designs are needed that include a healthy control group without PD.

\section{Etiology and pharmacological treatment of depression}

The average prevalence of major depressive disorder in PD is $17 \%$, whereas $35 \%$ of the patients suffer from clinically significant depressive symptoms. ${ }^{12}$ Depressive syndromes can negatively influence a patient's quality of life, activities of daily living (ADL), motor function, and cognitive functioning. ${ }^{12-15}$ In PD, research on risk factors for depression has primarily focused on disease-related factors, such as more severe motor symptoms, longer disease duration, advanced disease stages, reduced disease-related $A D L$, higher daily levodopa equivalent dose, and the presence of other non-motor symptoms such as hallucinations, sleep disturbances, and dysautonomia. ${ }^{16-19}$ The role of general risk factors nonspecific for PD, such as older age, female sex, a low level of education, physical disease, history of depression, smoking, alcohol abuse, positive and negative life events, personality, coping styles, and early childhood trauma, are less well understood. In Chapter 6, we used structural equation modeling (SEM) to explore the relative contribution of PD-specific and nonspecific risk factors in a model for depression in PD. Although PD-specific factors indeed appeared to be important markers for depression, the overall effect of non-specific factors on depression outcome appeared to be 3 times larger. This suggests that PD patients who develop depression possibly are those that have a preexisting vulnerability for depression due to the presence or constellation of general population risk factors for depression not related to PD. This may challenge the view that the pathophysiology of PD in itself leads to depressive symptoms, but rather that the PD pathophysiology may affect mood regulatory mechanisms or reduce compensatory mechanisms resulting in depression in patients with the highest pre-existing risk. This new approach may change our current view on etiology, prevention and treatment options for depression in PD.

In Chapter 7, we investigated which factors can predict improvement in depressive symptoms during antidepressant treatment in PD. Here, we found that PD patients with more severe depression and lower anxiety scores were more likely to show response to antidepressant treatment than those with milder depression and those with more severe anxiety. This is in line with results from treatment studies of depressed patients without PD. ${ }^{14,20,21}$ These results imply that depressed PD patients with more severe anxiety symptoms may require different or more intensive 
treatment. Other studies found evidence for a larger effect of antidepressant treatment in PD patients who used dopamine agonists or had a longer disease duration $^{13}$, and less improvement in patients in which postural instability was present pre-treatment. ${ }^{22}$ Again, this emphasizes the need to consider both disease-related factors and general factors not specific for PD when investigating depression in PD.

\section{Methodological Considerations}

\section{Diagnostic criteria}

The diagnosis of idiopathic PD was made with the use of the clinical operational criteria of the Queens Square Brain Bank (see Appendix). ${ }^{23,24}$ We did not perform additional assessments to verify this diagnosis, such as dopamine transporter single photon emission computed tomography (DAT-SPECT) scan in order to determine the availability of dopamine in the brain. As such, some patients may have been incorrectly diagnosed with idiopathic PD, and instead suffered from other causes of Parkinsonism. However, comparison with post-mortem histological findings showed that these operational criteria have a good diagnostic accuracy of $76 \%{ }^{24}$

\section{Study population}

The study described in Chapter 3 included PD patients who were recruited from the movement disorders clinic of the Maastricht University Medical Centre (MUMC). In Chapter 4 and 5, PD patients were recruited from the movement disorder clinics of both MUMC and the Lille University Medical Centre. The database from the study described in Chapter 6 included PD patients who were recruited from the movement disorders clinics and the neurology and psychiatry clinics from six centers in the United States, Europa, and Australia. Chapter 7 described a secondary analysis on the dataset of the randomized controlled trial Study of Antidepressants in PD (SADPD), in which subjects were recruited from 20 movement disorder clinics in the United States, Canada, and Puerto Rico.

In all studies, most patients exhibited mild-moderate severity of motor symptoms (Hoehn \& Yahr stage 1-3) and all patients participated on voluntary basis. This may have caused a selection bias with respect to the relatively low prevalence and mild levels of psychopathology in our samples, which may be higher in more advanced stages of the disease. 
Especially when investigating underlying neurobiological mechanisms of neuropsychiatric disorders in PD, it may be preferable to measure PD patients both on and off dopaminergic medication in order to accurately define and control for the role of dopamine. However, in neuroimaging studies this can be challenging as motor symptoms like tremor or dystonia can cause movement artifacts that may confound neuroimaging findings. However, it must be stressed that despite receiving dopamine replacement therapy (DRT) the patients in our samples still showed significant motor impairments, which indicates that the DRT did not fully compensate for the loss of dopamine. Alternatively, combining structural or functional MRI with more invasive methods such as PET or SPECT may provide important information about possible mediating effects of neurotransmitters with respect to neuropsychiatric disorders in PD.

\section{Study design}

The studies discussed in this thesis all have used a cross-sectional design, including the structural and functional MRI studies. The limitation of this type of design is that the study purposes can be merely descriptive and no conclusions can be drawn with respect to causality. A longitudinal study design would be necessary in order to draw firm conclusions on the progressive course of cognitive disorders in a specific subgroup of PD patients, on the predictive value of neuroanatomical markers and on risk factors for developing depressive disorders in PD. Longitudinal studies are very costly, though, and time-consuming. Moreover, especially in neuroimaging studies, scanner updates and new versions of software packages over time can be problematic. However, since the nature of our studies was mainly explorative, crosssectional designs are more useful when investigating possible associations that can be used in generating hypotheses for future research. ${ }^{25}$ Furthermore, we used advanced statistical methods, such as structural equation modeling (Chapter 6), that are considered valuable for estimating causal relationships. ${ }^{26}$

\section{Statistical methods and analyzing techniques}

Apart from descriptive statistics, chi-square tests, $t$-tests/analysis of variance (ANOVA), correlational analysis, and regression analysis, we also used more advanced techniques and statistical analyses, such as BOLD-fMRI (Chapter 3), cluster analysis (Chapter 4), optimized voxel-based morphometry (Chapter 5), and structural equation modeling (Chapter 6). 
We used task-related BOLD-fMRI in order to investigate differences between PD patients and healthy controls in functional activity related to emotional processing. The study was largely exploratory and hence we applied a whole brain approach with post hoc $\mathrm{ROI}$ analyses. A priori hypotheses about involved brain areas often include predefined regions of interest, which has the risk of missing less well-researched areas or possible compensatory activation. However, the BOLD signal is not a direct measure of neural activity, as it reflects hemodynamic changes for instance in the blood flow to brain regions that become more active during a task than regions with a lower BOLD response. ${ }^{27}$ Therefore, the functional MRI signal must always be interpreted with caution and the influence of factors such as noise, motion, and taskunrelated processing must always be taken into account.

In Chapter 4, we used confirmatory cluster analysis in order to validate the cognitive phenotypes that were derived from a previously applied explorative cluster analysis. ${ }^{7}$ In addition, factorial discriminant analysis assessed and supported the quality of the clusters' separation. Our five-cluster model was both statistically superior and clinically the most relevant and was not biased by predefined categories or a priori hypotheses. However, a drawback of using cluster analysis as a datadriven approach concerns the lack of control one has in defining the subgroups. As such, groups cannot be matched according to patient characteristics (e.g., age, level of education, gender) since these factors partially determine group membership. Moreover, our clusters were based on cognitive performance, which resulted in a low number of subjects for the two cognitively worst groups. For our neuroimaging analysis we had to merge these clusters for statistical power considerations, whereas the discriminant analysis clearly suggested differences in cognitive performance between the two clusters.

In Chapter 5, optimized VBM was used in order to explore differences in grey matter density between the patient subgroups defined in Chapter 4. VBM offers the possibility to apply automated whole brain analysis methods, which has the advantage of being less prone to subjectivity associated with ROI based methods and is not restricted by a priori assumptions about specific regions of interest. The disadvantage of using an automated approach concerns difficulties in detecting subtle changes/atrophy in areas that show high variability among elderly and diseased groups (e.g., the hippocampus) and may lead to misclassification of voxels due to reduced tissue contrast. Evaluating brain structure in neurodegenerative brains may profit from combining manual and automated approaches, for instance by 
creating a customized template from the acquired sample and use this adapted model brain for further automated analyzing steps.

Risk factors for developing depression in PD were investigated in Chapter 6 by using structural equation modeling (SEM). An important difference between SEM and more 'ordinary' regression models is its ability to estimate and test relationships among constructs rather than just testing the relation between an independent measure and the dependent outcome. However, one must always keep in mind that no temporal or causal conclusions van be drawn from a cross-sectional study. The nature of relationships can be hypothesized with SEM, but longitudinal studies are required to establish causality. Also, multiple regression models are often conducted in search for a model with acceptable fit. Although the original model is often based on theoretical assumptions, the revised models arise from statistical decisions and may deviate from the hypothesized model. On the other hand, this enables researchers to detect weaknesses in existing theories and models. ${ }^{28}$

\section{Clinical implications}

Our findings have several clinical implications. Our theory that medial prefrontal cortex activity may compensate or modulate emotion regulation in PD indicates that medial prefrontal cortex functioning may be an important target when treating affective disorders such as anxiety or depression in PD. Nonpharmacological treatments in patients with anxiety not suffering from PD such as Cognitive Behavioral Therapy (CBT) have been shown to restore impaired neuronal affective processing by increasing functional connectivity between limbic and prefrontal cortices. $^{29,30}$ Hence, PD patients who are cognitively intact may benefit from the preserved and potential compensatory involvement of medial prefrontal cortices in emotion regulation, despite the neurobiological deficits in affective circuitry related to the pathology of PD. Therapeutic interventions, such as CBT, that rely on frontal cognitive control mechanisms or emotion regulation strategies may therefore be particularly useful when treating affective disorders in this specific population. Future studies that measure functional connectivity patterns between frontal and subcortical limbic structures during emotional processing are warranted in order to confirm our hypothesis on increased compensatory prefrontal (top-down) cognitive control mechanisms in PD patients. 
Our study on neuroanatomical and neuropsychological markers of early cognitive decline in PD patients revealed a pattern of cerebral atrophy that could already be detected in PD patients with slowed mental speed but otherwise intact cognition. Clinicians should closely follow the course of decline in PD patients and offer them treatment options in an early phase in order to prevent further cognitive deterioration. Early identification of PD patients who are at risk of severe cognitive decline or conversion to dementia may offer treatment opportunities that can eventually delay the onset of dementia in these patients.

The large contribution of risk factors not related to PD in developing and treating depression may challenge the view that the pathophysiology of PD in itself leads to depressive symptoms. Instead the PD pathophysiology may affect mood regulatory mechanisms or reduce compensatory mechanisms resulting in depression in patients with the highest pre-existing risk. This new approach may change our current view on etiology, prevention and treatment options for depression in PD and emphasizes the need to consider both disease-related factors and general factors not specific for PD.

\section{FUTURE DIRECTIONS AND CONCLUDING REMARKS}

The findings of this thesis can provide several implications for future research on the etiology and neurobiology of emotional disturbances and cognitive decline in PD. Structural and functional neuroimaging approaches have appeared to be useful methods for exploring neural substrates of these non-motor symptoms. Researchers should however consider the use of explorative whole brain approaches (with post hoc analyses), rather than narrowing their area of interest beforehand according to previous findings. Although, this may be useful in order to replicate earlier findings, it may rule out the possibility to explore disturbances or even compensatory mechanisms elsewhere in the brain. In our fMRI study on emotional processing in PD we would probably have missed the potential compensatory medial prefrontal cortex activation if we had focused only on brain structures reported in previous studies (e.g., amygdala). Furthermore, future neuroimaging studies should consider combining multiple neuroimaging methods, such as functional MRI and Positron Emission Tomography (PET), as this could provide scientists with a better understanding of for instance the modulating effect of dopamine (or other neurotransmitters) on emotional processing in PD. 
In our study on cognitive disorders in PD, the combination of a data-driven approach to define groups and whole brain analysis turned out to be very useful for identifying early markers of cognitive decline in PD. However, future studies should longitudinally explore the extent to which neural substrates associated with early markers of cognitive impairment (e.g., slowed mental speed) can be used as a predictor of severe cognitive decline and conceivable conversion to dementia in PD. In addition, longitudinal studies can broaden our understanding of the contribution of specific neurochemical and neuropathological factors related to the course of cognitive decline in PD.

Future studies into the etiology of depression in PD should move their focus from solely PD-specific risk factors to a multifactorial framework involving both PDspecific and nonspecific risk factors. From a methodological perspective, SEM provides a useful statistical tool for verifying and possibly revising existing theories, yet, longitudinal studies are warranted to investigate true causality and assess the predictive value of specific risk factors. Nevertheless, both cross-sectional and longitudinal studies into depression, but also other psychopathological syndromes in PD, should not limit their focus to PD-specific factors as this may provide wrong conclusions about meaningful targets for prevention and treatment.

Finally, in this thesis we only discussed the neurobiology of emotional processing and cognitive disorders. However, another area in PD that urgently needs further research concerns the neurobiology and treatment of anxiety disorders. More than $35 \%$ of PD patients suffer from anxiety disorders, which have a negative effect on several motor symptoms including gait, dyskinesias, freezing, on/off fluctuations, and on quality of life in general. ${ }^{31,32}$ At present, there is no evidence-based treatment available. Progress in this area requires a better understanding of the underlying neurobiological mechanisms of anxiety in PD, which can provide the base for effective pharmacological or psychotherapeutic treatments options.

With this thesis we provided small pieces of the puzzle regarding the etiology and underlying neurobiology of emotion and cognition in PD. More research into this topic is of course needed to unravel the complete story. Nevertheless, I hope that our findings and recommendations can be valuable for the design of future studies and eventually for the development of effective treatment options that will contribute to an increase in quality of life of patients with Parkinson's disease and their caregivers. 


\section{REFERENCES}

1. Ochsner KN, Gross JJ. The Neural Architecture of Emotion Regulation. Handbook of emotion regulation 2007;1:87-109.

2. Phillips ML, Drevets WC, Rauch SL, Lane R. Neurobiology of Emotion Perception I: The Neural Basis of Normal Emotion Perception. Biolol Psych 2003;54:504-514.

3. Phillips ML, Ladouceur CD, Drevets WC. A Neural Model of Voluntary and Automatic Emotion Regulation: Implications for Understanding the Pathophysiology and Neurodevelopment of Bipolar Disorder. Molecular psychiatry 2008;13:833-857.

4. Gross JJ. Emotion Regulation. Handbook of emotions 2008;3:497-513.

5. Gross JJ. Antecedent-and Response-Focused Emotion Regulation: Divergent Consequences for Experience, Expression, and Physiology. Journal of personality and social psychology 1998;74:224.

6. Kehagia AA, Barker RA, Robbins TW. Neuropsychological and Clinical Heterogeneity of Cognitive Impairment and Dementia in Patients with Parkinson's Disease. The Lancet Neurology 2010;9:1200-1213.

7. Dujardin K, Leentjens AF, Langlois C, Moonen AJ, Duits AA, Carette AS, et al. The Spectrum of Cognitive Disorders in Parkinson's Disease: A Data-Driven Approach. Movement Disorders 2013;28:183-189.

8. Burton EJ, McKeith IG, Burn DJ, Williams ED, O'Brien JT. Cerebral Atrophy in Parkinson's Disease with and without Dementia: A Comparison with Alzheimer's Disease, Dementia with Lewy Bodies and Controls. Brain 2004;127:791-800.

9. Song SK, Lee JE, Park HJ, Sohn YH, Lee JD, Lee PH. The Pattern of Cortical Atrophy in Patients with Parkinson's Disease According to Cognitive Status. Movement Disorders 2011;26:289-296.

10. Weintraub D, Doshi J, Koka D, Davatzikos C, Siderowf AD, Duda JE, et al. Neurodegeneration across Stages of Cognitive Decline in Parkinson Disease. Archives of Neurology 2011;68:1562-1568.

11. Sabbagh MN, Adler CH, Lahti TJ, Connor DJ, Vedders L, Peterson LK, et al. Parkinson's Disease with Dementia: Comparing Patients with and without Alzheimer Pathology. Alzheimer disease and associated disorders 2009;23:295.

12. Reijnders JS, Ehrt U, Weber WE, Aarsland D, Leentjens AF. A Systematic Review of Prevalence Studies of Depression in Parkinson's Disease. Movement Disorders 2008;23:183-189.

13. Richard I, McDermott M, Kurlan R, Lyness J, Como P, Pearson N, et al. A Randomized, Double-Blind, Placebo-Controlled Trial of Antidepressants in Parkinson Disease. Neurology 2012;78:1229-1236.

14. Khan A, Leventhal RM, Khan SR, Brown WA. Severity of Depression and Response to Antidepressants and Placebo: An Analysis of the Food and Drug Administration Database. Journal of clinical psychopharmacology 2002;22:40-45.

15. Santangelo G, Vitale C, Trojano L, Longo K, Cozzolino A, Grossi D, et al. Relationship between Depression and Cognitive Dysfunctions in Parkinson's Disease without Dementia. Journal of neurology 2009;256:632-638.

16. Dissanayaka NN, Sellbach A, Silburn PA, O'Sullivan JD, Marsh R, Mellick GD. Factors Associated with Depression in Parkinson's Disease. Journal of affective disorders 2011;132:82-88. 
17. Riedel O, Heuser I, Klotsche J, Dodel R, Wittchen H-U. Occurrence Risk and Structure of Depression in Parkinson Disease with and without Dementia: Results from the Gepad Study. Journal of geriatric psychiatry and neurology 2009.

18. Becker C, Brobert G, Johansson S, Jick S, Meier C. Risk of Incident Depression in Patients with Parkinson Disease in the Uk. European Journal of Neurology 2011;18:448-453.

19. Gallagher DA, Schrag A. Psychosis, Apathy, Depression and Anxiety in Parkinson's Disease. Neurobiology of disease 2012;46:581-589.

20. Ising M, Lucae S, Binder EB, et al. A Genomewide Association Study Points to Multiple Loci That Predict Antidepressant Drug Treatment Outcome in Depression. Archives of General Psychiatry 2009;66:966-975.

21. Cohen A, Gilman S, Houck P, Szanto K, Reynolds C, III. Socioeconomic Status and Anxiety as Predictors of Antidepressant Treatment Response and Suicidal Ideation in Older Adults. Social Psychiatry and Psychiatric Epidemiology 2009;44:272-277.

22. Weintraub D, Taraborelli D, Morales KH, Duda JE, Katz IR, Stern MB. Escitalopram for Major Depression in Parkinson's Disease: An Open-Label, Flexible-Dosage Study. J Neuropsy Clin Neursci 2006;18:377-383.

23. De Rijk M, Rocca W, Anderson D, Melcon M, Breteler M, Maraganore D. A Population Perspective on Diagnostic Criteria for Parkinson's Disease. Neurology 1997;48:12771281.

24. Hughes AJ, Daniel SE, Kilford L, Lees AJ. Accuracy of Clinical Diagnosis of Idiopathic Parkinson's Disease: A Clinico-Pathological Study of 100 Cases. J Neurol Neurosurg Psychiatry 1992;55:181-184.

25. Levin KA. Study Design lii: Cross-Sectional Studies. Evid-based Dent 0000;7:24-25.

26. Anderson JC, Gerbing DW. Structural Equation Modeling in Practice: A Review and Recommended Two-Step Approach. Psychological bulletin 1988;103:411.

27. Thompson JK, Peterson MR, Freeman RD. Single-Neuron Activity and Tissue Oxygenation in the Cerebral Cortex. Science 2003;299:1070-1072.

28. Markus KA. Principles and Practice of Structural Equation Modeling by Rex B. Kline. Structural Equation Modeling: A Multidisciplinary Journal 2012;19:509-512.

29. Okai D, Samuel M, Askey-Jones S, David AS, Brown RG. Impulse Control Disorders and Dopamine Dysregulation in Parkinson's Disease: A Broader Conceptual Framework. European Journal of Neurology 2011;18:1379-1383.

30. Mansson KN, Carlbring P, Frick A, Engman J, Olsson C-J, Bodlund O, et al. Altered Neural Correlates of Affective Processing after Internet-Delivered Cognitive Behavior Therapy for Social Anxiety Disorder. Psychiatry Research: Neuroimaging 2013;214:229-237.

31. Leentjens AFG, Dujardin K, Marsh L, Richard IH, Starkstein SE, Martinez-Martin P. Anxiety Rating Scales in Parkinson's Disease: A Validation Study of the Hamilton Anxiety Rating Scale, the Beck Anxiety Inventory, and the Hospital Anxiety and Depression Scale. Movement Disorders 2011;26:407-415.

32. Leentjens AFG, Dujardin K, Pontone GM, Starkstein SE, Weintraub D, Martinez-Martin P. The Parkinson Anxiety Scale (Pas): Development and Validation of a New Anxiety Scale. Movement Disorders 2014;29:1035-1043.

33. Hughes AJ, Daniel SE, Kilford L, Lees AJ. Accuracy of Clinical Diagnosis of Idiopathic Parkinson's Disease: A Clinico-Pathological Study of 100 Cases. Journal of Neurology, Neurosurgery \& Psychiatry 1992;55:181-184. 


\section{APPENDIX}

\section{Queen Square Brain Bank criteria for Parkinson's disease ${ }^{23,33}$}

Step 1. Diagnosis of Parkinsonian Syndrome

- Bradykinesia (slowness of initiation of voluntary movement with progressive reduction in speed and amplitude of repetitive actions)

- At least one of the following:

- Muscular rigidity

- 4-6 Hz rest tremor

- Postural instability not caused by primary visual, vestibular, cerebellar, or proprioceptive dysfunction

Step 2. Exclusion criteria for Parkinson's disease

- History of repeated strokes with stepwise progression of parkinsonian features

- History of repeated head injury

- History of definite encephalitis

- Oculogyric crises

- Neuroleptic treatment at onset of symptoms

- More than one affected relative

- Sustained remission

- Strictly unilateral features after 3 years

- Supranuclear gaze palsy

- Cerebellar signs

- Early severe autonomic involvement

- Early severe dementia with disturbances of memory, language, and praxis

- Babinski sign

- Presence of cerebral tumor or communication hydrocephalus on CT scan

- Negative response to large doses of levodopa in absence of malabsorption

- MPTP exposure

Step 3. Supportive prospective positive criteria for Parkinson's disease

- Unilateral onset

- Rest tremor present

- Progressive disorder

- Persistent asymmetry affecting side of onset most

- Excellent response (70-100\%) to levodopa

- Severe levodopa-induced chorea

- Levodopa response for 5 years or more

- Clinical course of ten years or more 


\section{SUMMARY}


Parkinson's disease (PD) is one of the most prevalent neurodegenerative disorders among the elderly population, with an estimated prevalence of approximately $1 \%$ among people of 65 years or older that increases with age. Apart from the classical motor symptoms, such as bradykinesia, resting tremor, rigidity, and postural instability, many PD patients suffer from non-motor symptoms such as neuropsychiatric disorders, sleep disorders, autonomic symptoms, sensory symptoms, and cognitive disorders. Non-motor symptoms severely affect the quality of life of PD patients and their caregivers and have a negative impact on the prognosis of the disease. Although the impact of these disorders on a patient's health status has been widely recognized, treatments are still scarce and non-optimal. In order to develop effective interventions, both pharmacological and psychotherapeutic, it is essential that we first unravel the underlying neurobiological mechanisms of non-motor symptoms in PD. This thesis aimed to expand our understanding of neurobiological mechanisms for at least a subgroup of non-motor symptoms in PD, namely emotional disturbances and cognitive decline.

Chapter 1 is a general introduction into the theme and aims of this thesis. It provides information about the theoretical background of motor- and non-motor symptoms related to Parkinson's disease, as well as the research questions and outline of this thesis.

Chapter 2 provides a systematic review on the neurobiology of emotional processing deficits in PD. The included studies assessed both behavioral and neurobiological aspects of emotional processing and have investigated multiple aspects of emotional processing, such as facial emotion recognition, physiological arousal, and emotional prosody. Despite a large variation in methodology and clinical profiles among studies, the majority of studies reported that PD patients showed an intact ability to explicitly recognize and evaluate emotional stimuli. However, several studies that used structural magnetic resonance imaging (MRI), functional MRI or electroencephalogram (EEG), reported reduced grey matter density and altered brain activity in regions involved in implicit emotional processing, including the amygdala, anterior cingulate cortex, striatum, and prefrontal cortices. Implicit emotional processing appears to be associated with the involvement of a predominantly ventral system of affective neurocircuitry that is more strongly dependent on dopaminergic neurotransmission, and have been described to be affected in PD. Regions within the dorsal system, on the other hand can be more associated with cognitive and regulatory aspects of emotion, which seem to remain largely intact in PD patients. 
Following the results of the studies described in this review, PD patients are able to cognitively regulate or modulate their emotional responses despite reduced dopamine supplies, which can have important implications for the treatment of affective disorders in PD.

In Chapter 3 we cross-sectionally investigated implicit emotional processing and its functional correlates in PD patients and matched healthy control subjects using blood-oxygen-level dependent fMRI (BOLD-fMRI). We found reduced striatal activity but increased medial prefrontal activation and an intact ability in PD patients to explicitly recognize and evaluate emotional stimuli. While the reduced striatal activity is likely to be intimately related to the pathophysiology of PD, the increased medial prefrontal activation in PD patients may well underlie a compensatory topdown mechanism utilizing cognitive control to restore or modulate emotional processing at the behavioral level, despite striatal dysfunction. These results imply that therapeutic interventions for affective disorders that rely on cognitive control mechanisms may be particularly useful for PD patients who are cognitively intact, as they may benefit from a compensatory influence of prefrontal areas.

Chapter 4 explores the heterogeneity of the clinical presentation and progression of cognitive decline in PD. We used a data-driven approach in which patient subgroups were based on cognitive performance at a large battery of neuropsychological tests. In this cross-sectional study, we identified the following five cognitive profiles ranging from patients with performance in the normal range to patients with severe disorders in all cognitive domains. The groups differed in terms of age, apathy and frequency of hallucinations that were all higher in the clusters with mild or severe cognitive deficits, and the duration of formal education was lower in those groups.

In Chapter 5, we aimed to identify regional differences in grey matter (GM) atrophy between each of the cognitive phenotypes described in Chapter 4 in a prospective cohort study using structural MRI. Optimized voxel-based morphometry (VBM-Dartel) revealed a pattern of cerebral atrophy that could already be detected in PD patients with slight mental slowing but otherwise intact cognition. These patients showed increased GM atrophy in temporal, parietal, and frontal areas. Patients with a profile close to dysexecutive mild cognitive impairment showed increased GM atrophy in the parietal-temporal lobe, posterior cingulate, bilateral hippocampus, and bilateral amygdala. Patients with severe cognitive deficits in multiple domains showed a similar pattern of GM atrophy, with additional loss in the left medial 
temporal lobe and left parahippocampal gyrus. These results provide important support for the view that slowed mental speed and it's corresponding pattern of grey matter loss can be considered as an early neuroanatomical marker of cognitive decline in PD. Although age at evaluation appeared to be an important predictor of differences in GM loss, patients with severe cognitive deficits showed GM abnormalities unrelated to age. This may point to a more widespread Lewy body pathology with potential comorbid Alzheimer's disease pathology in these patients.

In Chapter 6, we used structural equation modeling (SEM) to explore the relative contribution of PD-specific factors (e.g., more severe motor symptoms, longer disease duration, advanced disease stages) and nonspecific risk factors (i.e., older age, female sex, lower education, history of depression) in a model for depression in PD. We found that PD-specific factors indeed appeared to be important markers for depression, yet the overall effect of nonspecific risk factors on depression outcome appeared to be three times larger. This suggests that PD patients who develop depression possibly are those that have a preexisting vulnerability for depression due to the presence or constellation of general population risk factors for depression not related to PD. This may challenge the view that the pathophysiology of PD in itself leads to depressive symptoms, but rather that the PD pathophysiology may affect mood regulatory mechanisms or reduce compensatory mechanisms resulting in depression in patients with the highest pre-existing risk.

In Chapter 7, we investigated which factors can predict improvement in depressive symptoms during antidepressant treatment in PD. Here, we found that PD patients with more severe depression and lower anxiety scores at baseline were more likely to show response to antidepressant treatment than those with milder depression and those with more severe anxiety. These findings have important implications for the treatment of depression in this population, as depressed PD patients with more severe anxiety symptoms may require different or more intensive treatment than those with no or mild anxiety.

Chapter 8 summarizes and discusses the main findings of all previous chapters in the light of current scientific research. Methodological issues are addressed and clinical implications and recommendations for future research are provided. 
SAMENVATTING 
De ziekte van Parkinson (ZvP) is een van de meest voorkomende neurodegeneratieve stoornissen bij ouderen, met een prevalentie van ongeveer $1 \%$ bij mensen van 65 jaar of ouder, die stijgt naarmate leeftijd toeneemt. Naast de klassieke motorische symptomen, zoals bradykinesie, rusttremor, rigiditeit en posturale instabiliteit, lijden veel parkinsonpatiënten aan zogenaamde nietmotorische symptomen zoals neuropsychiatrische stoornissen, slaapstoornissen, autonome symptomen, sensorische symptomen en cognitieve stoornissen. Nietmotorische symptomen hebben ernstige gevolgen voor de kwaliteit van leven van parkinsonpatiënten en hun omgeving en hebben een negatieve invloed op de prognose van de ziekte. Hoewel het effect van deze aandoeningen op de gezondheidstoestand van een patiënt algemeen is erkend, zijn effectieve behandelingen nog schaars. Om effectieve interventies te kunnen ontwikkelen, zowel farmacologisch als psychotherapeutisch, is het essentieel om allereerst de onderliggende neurobiologische mechanismen van niet-motorische symptomen bij de ZvP te onderzoeken. Dit proefschrift richt zich op het vergroten van onze kennis met betrekking tot de neurobiologische mechanismen van een subgroep van nietmotorische symptomen bij de ZvP, namelijk emotionele stoornissen en cognitieve achteruitgang.

Hoofdstuk 1 bevat een algemene inleiding in het onderwerp en de doelstellingen van dit proefschrift. Het hoofdstuk bevat tevens informatie over de theoretische achtergrond omtrent motorische- en niet-motorische symptomen bij de ZvP, evenals de onderzoeksvragen en opbouw van dit proefschrift.

Hoofdstuk 2 bevat een systematische review over de neurobiologie van verstoorde emotionele informatieverwerking bij de ZvP. De geïncludeerde studies onderzochten emotionele informatieverwerking zowel op gedragsniveau als op neurobiologisch niveau. Er werden bovendien meerdere aspecten van emotionele informatieverwerking onderzocht, zoals het herkennen van emotioneel geladen gezichtsuitdrukkingen, fysiologische 'arousal' of geprikkeldheid in reactie op emotionele stimuli, en het herkennen van emotionele prosodie (emotioneel geladen spraak). Ondanks dat deze studies een grote variatie in methodologie en klinische patiëntprofielen lieten zien, kwam uit de meeste studies naar voren dat parkinsonpatiënten geen afwijkingen lieten zien voor wat betreft het expliciet herkennen en evalueren van emotionele stimuli. Echter, een aantal studies toonden middels structurele Magnetic Resonance Imaging (MRI), functionele MRI of elektroencefalografie (EEG), een afname van grijze materie (grijze stof) in de hersenen van 
parkinsonpatiënten evenals afwijkende hersenactiviteit in gebieden die normaal gezien betrokken zijn bij impliciete emotionele informatieverwerking (zoals de amygdala, de anterieure cingulate cortex, het striatum en de prefrontale cortex). Deze defecten lijken met name voor te komen in het ventrale gedeelte van het neuronale emotionele circuit. Dit systeem is vooral betrokken bij impliciete emotionele informatieverwerking en is sterker afhankelijk van dopaminerge neurotransmissie. Het dorsale systeem, daarentegen, is meer betrokken bij expliciete emotionele informatieverwerking, namelijk de cognitieve en regulerende aspecten van emoties, welke grotendeels intact lijken te zijn bij parkinsonpatiënten. Concluderend laten de studies beschreven in deze review zien dat parkinsonpatiënten in staat zijn om emotionele reacties op gedragsniveau te herkennen en reguleren, ondanks de afname van dopamine in de hersenen van deze patiënten.

In Hoofdstuk 3 wordt een cross-sectionele studie beschreven waarin we impliciete emotionele informatieverwerking en de corresponderende neurobiologische correlaten hebben onderzocht bij parkinsonpatiënten en gematchte gezonde proefpersonen door middel van blood-oxygen-level dependent fMRI (BOLDfMRI). Bij parkinsonpatiënten vonden we enerzijds verminderde hersenactiviteit in het striatum, maar anderzijds een verhoogde activatie van de mediale prefrontale cortex. Daarnaast scoorden parkinsonpatiënten even goed als gezonde proefpersonen op een gedragstaak die expliciete herkenning en evaluatie van emotionele stimuli meet. De verminderde activiteit in het striatum is waarschijnlijk nauw verbonden met de pathofysiologie van de ZvP, terwijl de verhoogde mediale prefrontale activatie bij parkinsonpatiënten mogelijk duidt op een compensatiemechanisme waarmee emotionele informatieverwerking middels cognitieve controle (top-down) gemoduleerd kan worden, ondanks defecten in het emotionele systeem (striatum). Deze resultaten impliceren dat therapeutische interventies voor affectieve stoornissen waarbij gebruikt gemaakt wordt van deze cognitieve controlemechanismen bijzonder nuttig kunnen zijn voor parkinsonpatiënten waarvan de cognitieve functies nog voldoende intact zijn.

In Hoofdstuk 4 hebben we gekeken naar de heterogeniteit in klinische presentatie en progressie met betrekking tot cognitieve achteruitgang bij de ZvP. In een prospectieve studie hebben we patiëntsubgroepen kunnen creëren op basis van cognitieve prestaties op een uitgebreide neuropsychologische testbatterij. De cognitieve profielen van deze subgroepen varieerden van cognitieve prestaties 
binnen de normale range tot ernstige cognitieve achteruitgang in alle cognitieve domeinen. De groepen verschilden bovendien qua leeftijd, frequentie van apathie en hallucinaties, hetgeen hoger was in de clusters waarbij sprake was van milde tot ernstige cognitieve achteruitgang. Het opleidingsniveau was juist lager in deze groepen ten opzichte van de groepen zonder of met zeer lichte cognitieve achteruitgang.

Hoofdstuk 5 bevat een structureel beeldvormingsonderzoek naar verschillen in grijze stof atrofie tussen elk van de cognitieve fenotypes zoals beschreven in hoofdstuk 4. In een prospectieve cohortstudie hebben we door middel van optimalized voxel-based morphometry (VBM-Dartel) een patroon van cerebrale atrofie kunnen identificeren dat reeds zichtbaar was bij parkinsonpatiënten zonder cognitieve tekorten behoudens lichte mentale traagheid. De grijze stof atrofie bij deze patiënten was met name zichtbaar in temporale, pariëtale en frontale hersengebieden. Patiënten met een profiel dat sterk lijkt op een lichte cognitieve stoornis op het gebied van de executieve functies, vertoonden verhoogde grijze stof atrofie in de pariëtale-temporale kwab, in de posterieure gyrus cinguli, bilaterale hippocampus, en bilaterale amygdala. Patiënten met ernstige cognitieve tekorten in meerdere domeinen vertoonden een vergelijkbaar patroon van grijze stof atrofie, met bijkomend verlies in de linker mediale temporale kwab en linker parahippocampale gyrus. Deze resultaten tonen aan dat mentale traagheid en het bijbehorende patroon van grijze stof atrofie kunnen worden beschouwd als een vroege neuroanatomische marker van cognitieve achteruitgang bij de ZvP. Hoewel leeftijd bij evaluatie een belangrijke voorspeller bleek van verschillen in de mate en locatie van grijze stof atrofie, vonden we bij patiënten met ernstige cognitieve stoornissen grijze stof afwijkingen die niet gerelateerd waren aan leeftijd. Dit kan wijzen op meer diffuse Lewy body pathologie en mogelijk op co-morbide alzheimerpathologie bij deze patiënten.

In Hoofdstuk 6 hebben we 'structural equation modeling' (SEM) gebruikt om de relatieve bijdrage te onderzoeken van ziektespecifieke factoren (bijvoorbeeld ernstigere motorische symptomen, langere ziekteduur, meer gevorderd ziektestadium) en niet ziektespecifieke of algemene risicofactoren (bijvoorbeeld hogere leeftijd, vrouwelijk geslacht, lager opleidingsniveau, geschiedenis van depressie) in een model voor depressie bij de ZvP. We vonden dat ziektespecifieke factoren inderdaad belangrijke voorspellers waren voor de uitkomst op een depressievragenlijst. Echter, het totale effect van niet-specifieke risicofactoren voor 
depressie bleek drie keer zo groot te zijn. Dit suggereert dat het ontwikkelen van een depressie bij parkinsonpatiënten eerder verband houdt met een reeds bestaande kwetsbaarheid ten gevolge van algemene risicofactoren voor depressie, en niet zozeer verband houdt met factoren gerelateerd aan de ziekte zelf. Deze bevinding weerspreekt bovendien de visie dat depressie bij parkinsonpatiënten direct wordt veroorzaakt door de pathofysiologie van de ZvP. Parkinsonpatiënten waarbij reeds sprake is van algemene risicofactoren voor het ontwikkelen van een depressie, kunnen mogelijk wel eerder depressief worden wanneer neuronale stemmingsmechanismen of compensatiemechanismen aangetast worden door de ZvP.

In Hoofdstuk 7 hebben we gekeken naar factoren die een verbetering van depressieve symptomen kunnen voorspellen middels behandeling met antidepressiva bij depressieve parkinsonpatiënten. Hier vonden we dat parkinsonpatiënten met een ernstigere depressieve stoornis en geen of lichte angstklachten op baseline meer kans hadden op verbetering van depressieve symptomen middels antidepressiva dan patiënten met mildere depressieve klachten en ernstigere angstklachten. Deze resultaten hebben belangrijke implicaties voor de behandeling van depressie in deze populatie, aangezien depressieve parkinsonpatiënten met ernstigere angstsymptomen een andere of intensievere behandeling nodig hebben dan patiënten met geen of lichte angstklachten.

Hoofdstuk 8 geeft een overzicht van de hoofdbevindingen van dit proefschrift zoals beschreven in voorgaande hoofdstukken. Daarnaast worden methodologische aspecten besproken en klinische implicaties van de bevindingen gegeven. Tenslotte worden er aanbevelingen gedaan voor toekomstig onderzoek. 

KNOWLEDGE VALORIZATION 
The goal of this valorization paragraph is to describe how the knowledge resulting from the research in this thesis can be made valuable for clinical and societal use. This thesis focuses mainly on the etiology and neurobiological mechanisms of emotional disturbances and cognitive disorders in Parkinson's disease (PD).

\section{SOCIETAL RELEVANCE}

Non-motor symptoms of PD are now more and more recognized as being an integral part of the disease, with a significant impact on the quality of life of patients and their caregivers and leading to greater health care expenses. This thesis focuses on a subgroup of non-motor symptoms that are particularly disabling for patients: neuropsychiatric symptoms, and emotional disturbances and cognitive disorders in particular.

Although the impact of these disorders on a patient's health status has been widely recognized, treatments are still scarce and non-optimal. As such, the search for effective treatments is still ongoing. However, in order to develop effective interventions, both pharmacological and psychotherapeutic, it is essential that we first unravel the underlying neurobiological mechanisms of neuropsychiatric disorders in PD. The research in this thesis showed that a large network of subcortical limbic areas and cortical frontal areas is involved in emotional processing and that a disbalance in this system may lead to disturbances in emotion regulation. Taking into account the complexity and diversity of the disease, pharmacological and nonpharmacological treatments should be tailored to the needs and capabilities of PD patients in order to be effective. As such, knowledge about the neurobiological correlates of emotional dysfunction as well as potential compensation mechanisms in PD may provide an important base for successful treatment, as PD patients may for instance benefit from these compensational mechanisms with respect to emotion regulation. Consequently, successful treatment will contribute to an increase in quality of life of patients and caregivers, and eventually also to a decrease in health care costs and societal burden.

For cognition, we see a large spectrum of cognitive disorders where PD patients with different cognitive phenotypes show specific cognitive deficits. By exploring the neural correlates of these cognitive phenotypes we may be able to promptly identify patients who are at risk for developing more severe cognitive decline and PD dementia. As such, more specific and patient tailored pharmacotherapy and cognitive training options can be developed and provided in 
early stages of cognitive decline. Moreover, PD patients whose cognitive profile can be associated with comorbid Alzheimer's disease (AD) pathology may benefit from additional therapy directed at AD.

\section{TARGET AUDIENCE}

The results of this thesis are relevant for various target groups who are confronted with emotional disturbances and cognitive disorders in PD and it's consequences.

The results can be particularly important for health care professionals, such as neurologists, (neuro-)psychologists, psychiatrists and other clinicians who work with PD patients suffering from these symptoms. Information about the nature, risk factors and underlying causes of neuropsychiatric disorders will aid them in recognizing symptoms and early indicators of affective and cognitive disorders. As a result, they can provide patients and their caregivers with information about specific treatment options at an early stage. Also, they may consider regular MRI scans in PD patients who start to show signs of slight cognitive decline, and inform about early pharmacotherapy and cognitive training options that can help prevent further cognitive deterioration.

Furthermore, fellow scientists and clinicians who aim to develop new interventions for neuropsychiatric symptoms in $P D$ can use the results and recommendations made in this thesis. As stated before, the complexity and diversity of the disease requires pharmacological and non-pharmacological treatments that are tailored to the needs and capabilities of PD patients. Knowledge about the etiology and neurobiological correlates of neuropsychiatric disturbances in PD is very relevant to this respect.

Finally, results from this thesis can be valuable for researchers and clinicians who work with neurodegenerative diseases other than PD. Symptoms of anxiety, depression and apathy are for instance very common in Alzheimer's disease and other types of dementia, but also in other movement disorders such as Huntington's disease.

\section{Products / InNOVATION}

The research described in this thesis can be considered innovative in several ways. First, we applied an alternative approach of measuring and analyzing functional imaging data that enabled us to reveal for the first time potential compensatory activation during implicit emotional processing in PD patients. This finding can be 
particularly relevant for the development of new therapeutic interventions as PD patients may benefit from these compensatory mechanisms when being treated for affective disorders.

Another innovative approach within this thesis concerned the investigation of cognitive disorders in PD as a continuum rather than using diagnostic categories. This enabled us to revealed a pattern of cerebral atrophy that could already be detected in PD patients with slowed mental speed but otherwise intact cognition. Instead of searching for signs of mild cognitive impairment, clinicians should consider mental slowing as an early "red flag" for potential further cognitive decline. Hence, they should consider closely following the course of decline in these PD patients and offering them treatment options in an early phase in order to prevent further cognitive deterioration.

In order to explore the relative contribution of PD-specific and nonspecific risk factors in a model for depression in PD, we used an advanced statistical model that also takes into account mutual correlations among factors and their influence on the outcome. Unlike previous research and theories that focus on the effect of diseasespecific factors on the risk for developing depression in PD, this model showed us that it is equally important, if not more important, to take into account general risk factors for depression. This new approach may drastically change our current view on etiology, prevention and treatment options for depression in PD.

\section{IMPLEMENTATION}

The knowledge acquired from the studies in this thesis will be used for continuation of our research into underlying neurobiological mechanisms of neuropsychiatric disorders in PD. As became apparent from this thesis, PD patients show altered emotion regulation that can be partly associated with the progressive degeneration of dopaminergic systems that is characteristic for the disease. In addition, brains of PD patients deviate in general from healthy brains due to factors like cell death and other degenerative processes. Therefore, we aim to develop more advanced methods for analyzing imaging data from PD patients that take into account the neural alterations caused by the disease. Consequently, we intend to apply these methods for exploring another area in PD that urgently needs further research: anxiety disorders. With no evidence-based treatment available at present, it is again essential to first unravel the underlying neurobiological mechanisms of anxiety in PD. To this end, we will focus on functional connectivity patterns among areas within the neural emotional 
network. In addition, we aim to study structural and functional features of subregions of the amygdala, as they appear to play a central role in emotion regulation. Finally, we will use our findings for the development of a new psychotherapeutic intervention for anxiety in PD. To this end, we recently obtained a research grant from the Michael J. Fox Foundation for Parkinson's research to develop and investigate the clinical effectiveness of a specialized Cognitive Behavioural Therapy module for anxiety in PD. In addition, we will use structural and functional neuroimaging techniques to study changes in cerebral connectivity associated with successful treatment. Our main future goal is that this treatment module will be recognized and accepted as an effective non-pharmacological intervention for the treatment of anxiety symptoms in PD patients, and that it will find its way into widespread clinical use.

We further intend to follow-up the PD patients with different cognitive profiles in order to longitudinally explore the extent to which neural substrates associated with early markers of cognitive impairment (e.g., slowed mental speed) can be used as a predictor of severe cognitive decline and conceivable conversion to dementia in PD. Ultimately, this may open the door to early identification of PD patients who are at risk of severe cognitive decline or conversion to dementia and offers better treatment opportunities that can eventually delay the onset of dementia in these patients. 



\section{DANKWOORD}

ACKNOWLEDGMENTS 
Drie jaar lang lijkt het schrijven van het proefschrift nog zo ver weg. lets waar je nog lang niet mee bezig hoeft te zijn. En dan opeens ligt het er. Na een vliegensvlugge eindsprint kan het hoge woord er dan eindelijk uit: het is klaar! Een mooi moment om even terug te blikken op de afgelopen jaren en op al die mensen zonder wiens hulp dit proefschrift er nooit (op tijd) gekomen was.

Allereerst wil ik alle mensen bedanken die in de afgelopen jaren, soms meermaals, hebben deelgenomen aan mijn studies. Jullie waren een enorme bron van inspiratie en motivatie voor mij, en wisten mij er op de juiste momenten aan te herinneren waar ik het allemaal voor deed. Een diepe buiging voor jullie inzet en enthousiasme, en voor jullie bereidheid om een steentje bij te dragen aan dit belangrijke onderzoeksgebied.

Een speciaal woord van dank gaat uit naar mijn promotieteam, bestaande uit Frans Verhey en Albert Leentjens. Frans, bedankt dat je mij vier jaar geleden de kans hebt gegeven om de stap te zetten naar promotieonderzoek. Om mij enigszins mentaal voor te bereiden beschreef je dit traject destijds als 'Bloed, zweet en tranen'. Dat was het zeker, maar stiekem was toch ook wel heel leuk! Albert, bedankt voor je vertrouwen in mij, voor je kritische blik, en voor je inzet waarmee je mij door de vele obstakels van de afgelopen jaren heen hebt geholpen. Twee kritische blikken vormen niet altijd de makkelijkste weg, maar bereiken uiteindelijk wel het mooiste resultaat.

Ook wil ik graag de leden van de beoordelingscommissie bedanken voor het lezen en beoordelen van mijn proefschrift.

I would like to thank all co-authors of the articles in this thesis. It was a pleasure to collaborate and your valuable contribution to the quality of this thesis is highly appreciated.

Dr. Pontone and Dr. Bakker, dear Greg and Arnold, thank you very much for the opportunity to work with you at Johns Hopkins University. The experience to work in such a renowned lab has been of great value for my development as a researcher and as a person. I felt very welcome from the first day, for which I also have to thank your wonderful staff workers: Carrie, Aparna, Kate, and Leora. Thank you for all your help and for all the fun things we did during and after work. And of course my $\mathrm{JH}$ roomie: Tammy thank you so much for making me feel at home, for all your help, and for showing me the nice parts of Baltimore. Hopefully we will see each other again some day! 
Prof. dr. Dujardin, dear Kathy, thank you for the pleasant collaboration and your valuable input to my research and this thesis. I am pleased that our collaboration continues in such a wonderful new project.

Prof. Dr. Fink, Prof. dr. Weiss-Blankenhorn, thank you for collaborating with us in the seemingly infinite, but nevertheless finished research project about PD and emotional processing. Thank you, Michael and Ralph, for all your help and especially for your patience in teaching me about neuroimaging.

Ook dank aan iedereen die mij geholpen heeft bij de inclusie van patiënten voor mijn onderzoeken. Mirella Waber, Mayke Oosterloo, Mark Kuijf, Gerrit Tissingh, Peter van Domburg, Annelien Duits, excuus voor de herhaaldelijke 'spam' over het uitkijken naar mogelijke deelnemers, maar vooral ook enorm bedankt! En natuurlijk een oneindig woord van dank aan mijn stagiaires/redders in nood: Anke Wijers, Thera Reus en Niels de Graaf. Jullie hebben mij in de afgelopen jaren meer dan eens uit de brand geholpen en hebben daarmee een enorm belangrijke bijdrage geleverd aan de onderzoeken in dit proefschrift.

Verder wil ik graag alle (oud) collega's van de afdeling Psychiatrie en Neuropsychologie bedanken voor alle hulp en vooral voor de fijne werksfeer. De betrokkenheid naar elkaar is groot op onze afdeling, iets wat niet per se vanzelfsprekend hoeft te zijn in de onderzoekswereld. Nico, held, ik ben je eeuwig dankbaar voor als je reddingsacties en je hulp. Ze zeggen wel eens dat niemand onmisbaar is. Think again! Collega's van de afdeling Radiologie en Klinische Neurofysiologie, dank voor al jullie hulp en medewerking!

Behalve zelf een aantal keren van kamer te hebben gewisseld, zijn ook meerdere 'roomies' de revue gepasseerd. Jennifer, bedankt dat je me in het begin zo goed op weg hebt geholpen en voor alle gezelligheid. Olga, jij hebt me misschien wel op mijn meest warrige momenten meegemaakt (een tandenborsteloplader aanzien voor een laptopoplader??). Gelukkig hebben we ook veel lol gehad en heb ik veel gehad aan je wijze raad (werk- en niet werk gerelateerd). Inez, van jou heb ik nog niet zo lang geleden afscheid moeten nemen als kamergenootje. Wat hebben we samen gelachen om alle stommiteiten van onszelf (en van anderen..). Gelukkig zit je maar een paar deuren verder en kan ik af en toe nog eens binnenlopen voor advies en vooral veur get sjtomme kal. Mijn nieuwe roomies, Lisa en Nikos, een gezellige drukke boel is het meestal bij ons. Hopelijk kunnen we elkaar nog veel neuroimaging wijsheid bijbrengen de komende tijd! En dan last but not least, roomie \#1 Liselot, wat een supergezellige, leuke, lieve meid ben jij. Zo fijn dat ik met jou zowel mijn 
frustraties als mijn crush voor Tom Smith kan delen, als ook mijn stuitermomenten en alles wat daar bij hoort. Gelukkig blijven we nog gezellig een aantal jaartjes tegenover elkaar zitten! Geen roomie, maar wel een lieve buurvrouw, Syenna. Turbulente jaren kunnen we het wel noemen, met gelukkig ook veel mooie en hilarische momenten. Ik verheug me op de tijd die komen gaat, twee kleine hummelkes, die hopelijk net zo'n lieve vriendjes worden als wij!

Lieve paranimfjes, Lizzy en Joany, lieve Rosalie, wat ben ik blij dat ik jullie heb leren kennen. Ik heb het geloof ik al heel vaak gezegd, maar zonder jullie was ik al minstens 18 keer gestorven in de afgelopen paar jaar. Ik vind het heel bijzonder hoe we er voor elkaar geweest zijn, hebben gelachen, geklaagd, gehuild, nog meer gelachen en vooral ontzettend veel lol gehad met elkaar. Met name Florence gaat wat mij betreft de boeken in als de meest hilarische 'vakantie' ooit. Ik heb er een paar hele lieve vriendinnetjes bij gekregen. Dankjewel voor al jullie steun en mental support. Hopelijk gaan we samen nog heel veel spritzer avondjes beleven (met of zonder karaoke...)! En natuurlijk gaan we een glansrijke carrière tegemoet bij Dr. T!

Anouscha, bedankt voor de fijne samenwerking in het afgelopen jaar. Samen met onze achterban hebben we toch maar iets heel moois neergezet. Parkinson Café Maastricht is een feit en blijft hopelijk nog lang evenzo succesvol en gezellig!

Lieve vriendjes en vriendinnetjes, lieve (schoon)familie, dank voor jullie interesse en steun in de afgelopen jaren. Het was af en toe misschien een mysterie waar ik nu eigenlijk mee bezig was, maar desondanks wisten jullie toch de benodigde mental support te geven. Lieve papa en mama, jullie bijdrage aan mijn ontwikkeling in de afgelopen jaren is van onschatbare waarde geweest. Dankjewel, voor álles.

Een laatste woord van dank aan jou lief, mijn verloofde, mijn rustpunt en enorme steun. Jij weet als geen ander hoe ik dit traject beleefd heb. Aan een 'zonder jou..' ga ik niet eens beginnen. Mèt jou heb ik alle behaalde deadlines en andere succesmomenten doorgemaakt, maar ook alle kommer en kwel. Samen hebben we de mooiste reizen gemaakt, avonturen beleefd en een prachtig thuis gecreëerd. Maar vooral ook gebouwd aan onze toekomst. Nog eventjes geduld, en dan mogen we beginnen aan een nieuw avontuur, wij samen. 
CuRriculum VitaE 


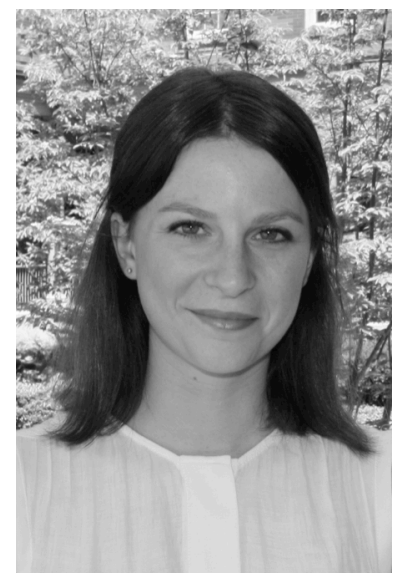

Anja Moonen was born on May 271987 in Geleen ('de Wereldstad'). After graduating from high school in 2005 she started to study Psychology and Neuroscience at Maastricht University. In 2008 she started her master in Neuropsychology, for which she performed a research and clinical internship at the Department of Psychiatry and Neuropsychology of the Maastricht University Medical Centre (MUMC). After she received her Master of Science degree for Psychology and Neuroscience in 2010, she started working as a psychologist in the same department. Before diving into the academic world she temporarily left Maastricht to learn French in Paris, France, and to experience the Parisian culture. Upon her return in 2011, Anja started working as a PhD candidate at the department of Psychiatry and Neuropsychology under supervision of Prof. dr. Frans Verhey and Dr. Albert Leentjens. This resulted in the work described in the current thesis. Within the scope of her PhD trajectory, she has worked as a visiting scientist at the Research Institute Jülich, Germany, where she did an fMRI study into the neurobiology of emotional processing in Parkinson's disease. During the final year of her PhD project, Anja did a short-term scholarship at the department of Psychiatry and Behavioral Sciences, division of Psychiatric Neuroimaging at the Johns Hopkins University School of Medicine (Baltimore, USA). Here she investigated neural correlates of anxiety in Parkinson's disease by using high-resolution structural and functional neuroimaging techniques. She is founding member of the Parkinson support group in Maastricht and contact person in Maastricht for the Dutch Parkinson Patient Foundation (Parkinsonvereniging). Recently, Anja obtained a research grant from the Michael J. Fox foundation in collaboration with Dr. Leentjens en Prof. dr. Kathy Dujardin, that allowed her to continue her research into Parkinson's disease as a postdoctoral researcher at Maastricht University. 
Publications 
Dujardin K, Leentjens AFG, Langlois C, Moonen, AJH, Duits AA, Carette AS, Duhamel A. (2013). The spectrum of cognitive disorders in Parkinson's disease: a data-driven approach. Movement Disorders, 28(2), 183-189.

Leentjens AFG, Moonen AJH, Dujardin K, Marsh L, Martinez-Martin P, Richard IH, Starkstein SE, Köhler S. (2013). Modeling depression in Parkinson disease: disease-specific and nonspecific risk factors. Neurology, 81(12), 1036-1043.

Moonen AJH, Wijers A, Leentjens AFG, Christine CW, Factor S, Juncos, JL, Lyness JM, Marsh L, Panisset M, Pfeiffer R, Rottenberg D, Serrano Ramos C, Shulman L, Singer C, Slevin J, McDonald W, Auinger P, Richard I. (2014). Severity of depression and anxiety are predictors of response to antidepressant treatment in Parkinson's disease. Parkinsonism \& Related Disorders, 20(6), 644-646.

Broen MPG, Moonen AJH, Kuijf ML, Dujardin K, Marsh L, Richard IH, Starkstein SE, Martinez-Martin P, Leentjens AFG. (2015). Factor analysis of the Hamilton Depression Rating Scale in Parkinson's disease. Parkinsonism \& Related Disorders 21(2), 142-6.

Dujardin K, Moonen AJH, Behal H, Defebvre L, Duhamel A, Duits AA, Plomhause L, Tard C, Leentjens AFG. (2015). Cognitive disorders in Parkinson's disease: confirmation of a spectrum of severity. Parkinsonism \& Related Disorders, 21(11), 1299-1305.

Broen MPG, Köhler S, Moonen AJH, Kuijf ML, Dujardin K, Marsh L, Richard IH, Starkstein SE, Martinez-Martin P, Leentjens AFG. (2015). Modeling anxiety in Parkinson's disease. Movement Disorders (in press).

Moonen AJH, Wijers A, Dujardin K, Leentjens AFG. Neurobiological correlates of emotional processing in Parkinson's disease: a systematic review of experimental studies. Submitted.

Moonen AJH, Weiss PH, Wiesing M, Weidner R, Fink GR, Reijnders JSAM, Weber WM, Leentjens AFG. Emotional processing in Parkinson's disease: can medial prefrontal activation compensate for striatal dysfunction? Submitted. 
Moonen AJH, Dujardin K, Behal H, Defebvre L, Duhamel A, Duits AA, Plomhause L, Tard C, Hofman P, van Bussel F, Leentjens AFG. Reduced grey matter density associated with early cognitive decline in Parkinson's disease: a voxel-based morphometry study. Submitted. 



\section{THESIS DEFENSES FROM MHENS}

School for Mental Health and Neuroscience 
2013

Rob Havermans: Bipolar disorder in daily life; Mood and cortisol responses to naturally occurring events. Supervisor: Prof.dr. M. de Vries; Co-Supervisor: Dr. N. Nicolson. Véronique Moers-Hornikx: Deep brain stimulation and the cerebellum.

Supervisors: Prof.dr. J. Vles / Prof.dr. Y. Temel; Co-Supervisor: Dr. G. Hoogland.

Nicole Veldhorst-Janssen: Intranasal delivery of rapid acting drugs.

Supervisors: Prof.dr. M. Marcus / Prof.dr. C. Neef; Co-Supervisor: Dr. P.H. van der Kuy.

Stéphanie Knippenberg: Vitamin D and Multiple Sclerosis: immunological and clinical outcome.

Supervisor: Prof.dr. J. Cohen-Tervaert; Co-Supervisors: Dr. J. Damoiseaux/Dr. Y. Bols.

Erik D. Gommer: Dynamic Cerebral Autoregulation: from methodology towards clinical application.

Supervisors: Prof.dr. W.H. Mess / Prof.dr. R.B. Panerai, UK; Co Supervisor: Dr.ir. J.P.H. Reulen.

Olga A.H. Reneerkens: Can PDE inhibition improve cognition? Translational insights.

Supervisor: Prof.dr. H.W.M. Steinbusch; Co-Supervisor: Dr. J. Prickaerts.

Lyzel S. Elias-Sonnenschein: Clinical and biomarker correlates of genetic risk factors for

Alzheimer's disease. Supervisor: Prof.dr. F.R.J. Verhey; Co-Supervisor: Dr. P.J. Visser.

Diego F. Mastroeni: Epigenetic Dysregulation and the Pathophysiology of of Alzheimer's

Disease. Supervisors: Prof.dr. H.W.M. Steinbusch / Prof.dr. P.D. Coleman, Sun City,

Arizona; Co-Supervisors: Dr. B.P.F. Rutten / Dr. D.L.A. van den Hove.

Leonidas Chouliaras: Epigenetic Regulation in Aging and Alzheimer's disease: A

translational perspective. Supervisor: Prof.dr. H.W.M. Steinbusch; Co-Supervisors:

Dr. B.P.F. Rutten / Dr. D.L.A. van den Hove.

Liesbeth Knaepen: Perinatal events and altered pain sensitivity in later life.

Supervisors: Prof.dr. E.A.J. Joosten / Prof.dr. D. Tibboel, EUR; Co-Supervisor: Dr. J. Patijn.

Marisela Martinez-Claros: Hippocampal plasticity and corticosterone: From dendrites to behaviour. Supervisor: Prof.dr. H.W.M. Steinbusch; Co-Supervisors: Dr. J.L. Pawluski /Dr. J. Prickaerts.

Marcus D. Lancé: A circle of improvement in bleeding management: from laboratory to clinic and back. Supervisors: Prof.dr. M.A.E. Marcu / Prof.dr. J.W.M. Heemskerk; CoSupervisor: Dr. Y.M.C. Henskens.

Hilde Braakman: Imaging the brain; neuronal correlates of cognitive impairment in children with frontal lobe epilepsy. Supervisors: Prof.dr. A.P. Aldenkamp/Prof.dr. J.S.H. Vles; Co-Supervisors: Dr.ir. W.H. Backes / Dr. P.A.M. Hofman.

Willem H. van Zwam: Aneurysmal subarachnoid hemorrhage: imaging strategies and cost- 
effectiveness aspects in diagnostic work-up and post-therapeutic follow-up. Supervisors: Prof.dr. J.T. Wilmink / Prof.dr. J.E. Wildberger; Co-Supervisor: Dr. P.A.M. Hofman.

Klara De Cort: The Pathogenesis of Panic Disorder. Supervisors: Prof.dr. I. Myin-Germeys /

Prof.dr. E.J.L. Griez; Co-Supervisors: Dr. K.R.J. Schruers / Dr. I. Van Diest, Leuven. Kim van Wijck: Mind the Gap; experimental studies on splanchnic hyperfusion and gastrointestinal integrity loss in man. Supervisors: Prof.dr. W.A. Buurman / Prof.dr. C.H.C. Dejong; Co-Supervisor: Dr. K. Lenaerts.

Yvette Roke: Antipsychotic-induced hyperprolactinemia in children and adolescents with mainly autism spectrum disorders. Prevalence, symptoms, clinical consequences and genetic risk factors.

Supervisors: Prof.dr. P.N. van Harten/Prof.dr. J.K. Buitelaar (RUN); Co-Supervisor: Dr. A. Boot (UMCG).

Fleur Goezinne: Retinal detachment surgery: pre and postoperative prognostic factors.

Supervisors: Prof.dr. F. Hendrikse / Prof.dr. C.A.B. Webers; Co-Supervisor: Dr. E.C. La Heij (Amsterdam).

Ralph L.J.G. Maassen: The Merits of Videolaryngoscopy during Glottic Visualisation for Endotracheal Intubation. Supervisors: Prof.dr. M. Marcus / Prof.dr. A. van Zundert (University of Queensland).

Maria J. de Sousa Guerreiro: The role of sensory modality in age-related distraction.

Supervisor: Prof.dr. C.M. van Heugten; Co-Supervisor: Dr. P.W.M. van Gerven. Ine Rayen: Effects of developmental fluoxetine exposure on neurobehavioral outcomes. Supervisor: Prof.dr. H.W.M. Steinbusch; Co-Supervisors: Dr. J.L. Pawluski/Dr. T.D. Charlier (Ohio University, USA).

Nynke M.G. Bodde: Psychogenic non-epileptic seizures; a separate disorder or part of a continuum?

Supervisors: Prof.dr. R. van Oostenbrugge / Prof.dr. K. Vonck (UZ Gent); Co-Supervisors:

Dr. R. Lazeron / Dr. A. de Louw (Epilepsiecentrum Kempenhaeghe, Heeze).

Alejandro M. Gomez: Novel strategies for making myasthenia less gravis: targeting plasma cells and the neuromuscular junction. Supervisor: Prof.dr. M.H. De Baets; CoSupervisors: Dr. M. Losen/Dr. P. Martinez-Martinez.

Mohammad S. Rahnama'i: Prostaglandins and Phosphodiesterases in the Urinary Bladder

Wall. Supervisors: Prof.dr. Ph. Van Kerrebroeck / Prof.dr. S. de Wachter (Universiteit Antwerpen); Co-Supervisor: Dr. G. van Koeveringe.

Mariken B. de Koning: Studying biomarkers in populations at genetic and clinical high risk for psychosis. Supervisors: Prof.dr. T. Amelsvoort / Prof.dr. J. Booij (AMC).

Fabien Boulle: Epigenetic regulation of BDNF/TrkB signaling in the pathophysiology and 
treatment of mood disorders. Supervisors: Prof.dr. H.W.M. Steinbusch/Prof.dr. L. Lanfumey (Universiteit Parijs); Co-Supervisors: Dr. D. van den Hove/Dr. G. Kenis.

2014

Iris Nowak-Maes: Tinnitus; assessment of quality of life \& cost-effectiveness.

Supervisors: Prof.dr. M. Peters / Prof.dr. B. Kremer; Co-Supervisors: Dr. M. Joore /

Dr. L. Anteunis.

Marjolein Huijts: Cognitive function in patients with cerebral small vessel disease.

Supervisor: Prof.dr. R.J. van Oostenbrugge; Co-Supervisors: Dr. A.A. Duits / Dr. J. Staals.

Markus Gantert: Fetal inflammatory injury as origin of long term disease: Lessons from animal models. Supervisors: Prof.dr. B. Kramer / Prof.dr. L. Zimmermann; CoSupervisor: Dr. A. Gavilanes.

Elke Kuypers: Fetal development after antenatal exposures: Chorioamnionitis and maternal glucocorticoids. Supervisors: Prof.dr. B.W. Kramer / Prof.dr. H.W. Steinbusch / Prof.dr. Suhas G. Kallapur (University of Cincinnati, Ohio, USA).

Pieter Kubben: Ultra low-field strength intraoperative MRI for Glioblastoma Surgery.

Supervisor: Prof.dr. J.J. van Overbeeke; Co-Supervisor: Dr. H. van Santbrink. Laura Baijens: Surface electrical stimulation of the neck for oropharyngeal dysphagia in Parkinson's disease: therapeutic aspects and reliability of measurement.

Supervisor: Prof.dr. B. Kremer; Co-Supervisor: Dr. R. Speyer, Townsville.

Janneke Hoeijmakers: Small fiber neuropathy and sodium channels; a paradigm shift.

Supervisor: Prof.dr. R.J. van Oostenbrugge; Co-Supervisors: Dr. C.G. Faber / Dr. I.S.J. Merkies.

Stephanie Vos: The Role of biomarkers in preclinical and prodromal Alzheimer's disease.

Supervisor: Prof.dr. F.R. Verhey; Co-Supervisor: Dr. P.J. Visser.

Muriël Doors: The Value of Optical Coherence Tomography in Anterior Segment Surgery.

Supervisors: Prof.dr. R.M. Nuijts / Prof.dr. C.A. Webers; Co-Supervisor: Dr. T.T.J.M. Berendschot.

Anneke Maas: Sleep problems in individuals with genetic disorders associated with intellectual disability. Supervisors: Prof.dr. I. Curfs / Prof.dr. R. Didden.

Sebastiaan van Gorp: Translational research on spinal cord injury and cell-based therapies;

a focus on pain and sensorimotor disturbances. Supervisors: Prof.dr. B. Joosten /

Prof.dr. M. van Kleef; Co-Supervisors: Dr. J. Patijn /Dr. R. Deumens, KU Leuven.

Andrea Sannia: High risk newborns and brain biochemical monitoring.

Supervisor: Prof.dr. J.S.H. Vles; Co-Supervisors: Dr. D. Gazzolo, Alessandria, Italy I

Dr. A.W.D. Gavilanes. 
Julie A.D.A. Dela Cruz: Dopamine mechanisms in learning and memory: Evidence from rodent studies. Supervisors: Prof.dr. H.W.M. Steinbusch/Prof.dr. R.J. Bodnar, New York; Co-Supervisor: Dr. B.P.F. Rutten.

René Besseling: Brain wiring and neuronal dynamics; advances in MR imaging of focal epilepsy. Supervisors: Prof.dr. A.P. Aldenkamp / Prof.dr.ir. W.H. Backes; Co-Supervisor: dr. J.F.A. Jansen.

Maria Quint-Fens: Long-term care after stroke; development and evaluation of a long-term intervention in primary care. Supervisors: Prof.dr. J.F.M. Metsemakers / Prof.dr. C.M. van Heugten / Prof.dr. M. Limburg, Almere; Co-Supervisor: dr. G.H.M.I. Beusmans.

Veronique Moulaert: Life after survival of a cardiac arrest; the heart of the matter.

Supervisors: Prof.dr. J.A. Verbunt / Prof.dr. C.M. van Heugten / Prof.dr. D.T. Wade, Oxford, UK.

Feikje Smeets: The hallucinatory-delusional state: a crucial connection in the psychosis symptom network. Supervisor: Prof.dr. J. van Os; Co-Supervisor: Dr. T. Lataster.

Lies Clerx: Alzheimer's disease through the MR-eye; novel diagnostic markers and the road to clinical implementation". Supervisor: Prof.dr. F. Verhey; Co-Supervisors:

Dr. P.J. Visser / P. Aalten.

Sonny Tan: The subthalamic nucleus in Parkinson's disease. Supervisors: Prof.dr. Y. Temel/ Prof.dr. H.W.M. Steinbusch / Prof.dr. T. Sharp, Oxford, UK / Prof.dr. V. VisserVandewalle, Koln.

Koen van Boxem: The use of pulsed radiofrequency in the management of chronic lumbosacral radicular pain. Supervisors: Prof.dr. M. van Kleef / Prof.dr. E.A.J. Joosten; Co-Supervisor: Assoc. Prof.dr. J. van Zundert.

Jérôme Waterval: Hyperostosis cranialis interna. Supervisors: Prof.dr. J.J. Manni / Prof.dr. R.J. Stokroos.

Sylvie Kolfschoten-van der Kruijs: Psychogenic non-epileptic seizures; the identification of neurophysiological correlates. Supervisors: Prof.dr. A.P. Aldenkamp/Prof.dr. K.E.J. Vonck, Universiteit Gent; Co-Supervisors: Dr. J.F.A. Jansen / Dr. R.H.C. Lazeron, Kempenhaeghe.

Wouter Pluijms: Spinal cord stimulation and pain relief in painful diabetic: polyneuropathy, a translational approach. Supervisors: Prof.dr. M. van Kleef / Prof.dr. E.A. Joosten; Co-supervisor: Dr. C.G. Faber.

Ron Handels: Health technology assessment of diagnostic strategies for Alzheimer's disease. Supervisors: Prof.dr. F.R.J. Verhey / Prof.dr. J.L. Severens (EUR); Co-Supervisor: Dr. M.A. Joore / Dr. C.A.G. Wolfs.

Evelyn Peelen: Regulatory T cells in the pathogenesis of Multiple Sclerosis: potential targets for vitamin D therapy. Supervisors: Prof.dr. R.M.M. Hupperts / Prof.dr. J.W. 
Cohen Tervaert; Co-Supervisor: Dr. J.G.M.C. Damoiseaux / Dr. M.M.G.L.Thewissen, Diepenbeek.

Reint Jellema: Cell-based therapy for hypoxic-ischemic injury in the preterm brain.

Supervisors: Prof.dr. B.W.W. Kramer/Prof.dr. H.W.M. Steinbusch; Co-Supervisor:

Dr. W.T.V. Germeraad/Dr. P. Andriessen, Veldhoven.

Maria Wertli: Prognosis of Chronic Clinical Pain Conditions: The Example of Complex

Regional Pain Syndrome 1 and Low Back Pain. Supervisors: Prof.dr. M. van Kleef;

Co-Supervisor: Dr. F. Brunner, Zürich / Dr. R. Perez, VUmc.

Dagmar Zeef: An experimental model of Huntington's disease: Validation \& Stimulation.

Supervisors: Prof.dr. Y. Temel / Prof.dr. H.W.M. Steinbusch; Co-supervisor: Dr. A. Jahanshahi.

Jeroen Decoster: Breaking Down Schizophrenia into phenes, genes and environment.

Supervisors: Prof.dr. I. Myin-Germeys / Prof.dr. M. De Hert, KU Leuven; Co-Supervisor:

Dr. R. van Winkel.

Eaja Anindya Sekhar Mukherjee: Fetal Alcohol Spectrum Disorders: exploring prevention

and management. Supervisor: Prof.dr. L.M.G. Curfs; Co-Supervisor: Prof. S. Hollins, St.

George's University of London, UK.

Catherine van Zelst: Inside out; On stereotype awareness, childhood trauma and stigma in psychosis. Supervisors: Prof.dr. Ph. Delespaul / Prof.dr. J. van Os.

Ibrahim Tolga Binbay: Extended Psychosis Phenotype in the Wider Social Environment.

Supervisor: Prof.dr. J. van Os; Co-Supervisor: Dr. M. Drukker.

Frank Van Dael: OCD matters in psychosis. Supervisors: Prof.dr. J. van Os / Prof.dr. I. MyinGermeys.

Pamela Kleikers: NOXious oxidative stress: from head toe too and back.

Supervisors: Prof.dr. H.H.H.W. Schmidt / Prof.dr. H.W.M. Steinbusch; Co-Supervisor:

Dr. B. Janssen.

José Luis Gerardo Nava: In vitro assay systems in the development of therapeutic interventions strategies for neuroprotection and repair. Supervisors: Prof.dr.med. J.

Weis / Prof.dr. H.W.M. Steinbusch; Co-Supervisor: Dr. G.A. Brook, RWTH Aachen.

Eva Bollen: Cyclic nucleotide signaling and plasticity. Supervisors: Prof.dr. H.W.M.

Steinbusch / Prof.dr. R. D'Hooge, KU Leuven; Co-Supervisor: Dr. J. Prickaerts.

2015

Jessica A. Hartmann: A good laugh and a long sleep; Insights from prospective and ambulatory assessments about the importance of positive affect and sleep in mental health. Supervisor: Prof.dr. J. van Os; Co-Supervisors: C.J.P. Simons / Dr. M. Wichers. 
Bart Ament: Frailty in old age; conceptualization and care innovations.

Supervisors: Prof.dr. G.I.J.M. Kempen / Prof.dr. F.R.J. Verhey; Co-Supervisor: Dr. M.E. de Vugt.

Mayke Janssens: Exploring course and outcome across the psychosis-continuum.

Supervisor: Prof.dr. I. Myin-Germeys; Co-Supervisor: Dr. T. Lataster.

Dennis M.J. Hernau: Dopayours is not dopamine: genetic, environmental and pathological variations in dopaminergic stress processing. Supervisor: Prof.dr. I. Myin-Germeys;

Co-Supervisors: Prof.dr. F.M. Mottaghy / Dr. D. Collip.

Ingrid M.H. Brands: The adaptation process after acquired brain injury Pieces of the puzzle.

Supervisors: Prof.dr. C.M. van Heugten / Prof.dr. D.T. Wade, Oxford UK; Co-Supervisors:

Dr. S.Z. Stapert / Dr. S. Köhler.

Francesco Risso: Urinary and salivary S100B monitoring in high risk infants.

Supervisor: Prof.dr. J.S.H. Vles; Co-Supervisors: Dr. D. Gazzolo, Genoa,Italy / Dr. A.W.D. Gavilanes.

Alessandro Borghesi: Stem and Progenitor Cells in Preterm Infants: Role in the

Pathogenesis and Potential for Therapy. Supervisor: Prof.dr. L. Zimmermann; Prof.dr.

B. Kramer; Co-Supervisors: Dr. D. Gazzolo, Genoa,Italy / Dr. A.W.D. Gavilanes.

Claudia Menne-Lothmann: Affect dynamics; A focus on genes, stress, and an opportunity

for change.

Supervisor: Prof.dr. J. van Os; Co-Supervisors: Dr. M. Wichers / Dr. N. Jacobs.

Martine van Nierop: Surviving childhood new perspectives on the link between childhood

trauma and psychosis. Supervisors: Prof.dr. I. Myin-Germeys / Prof.dr. J. van Os; Co-

Supervisor: Dr. R. van Winkel.

Sylvia Klinkenberg: VNS in children; more than just seizure reduction.

Supervisors: Prof.dr. J. Vles / Prof.dr. A. Aldenkamp; Co-Supervisor: Dr. H. Majoie.

Anouk Linssen: Considerations in designing an adult hearing screening programme.

Supervisor: Prof.dr. B. Kremer; Co-Supervisors: Dr. L. Anteunis / Dr. M. Joore.

Janny Hof: Hearing loss in young children; challenges in assessment and intervention.

Supervisors: Prof.dr. B. Kremer/Prof.dr. R. Stokroos / Prof.dr. P. van Dijk, RUG; Co-Supervisor:

Dr. L. Antheunis.

Kimberly Cox-Limpens: Mechanisms of endogenous brain protection; Clues from the

transcriptome. Supervisors: Prof.dr. J. Vles / Prof.dr. L. Zimmermann; Co-Supervisor:

Dr. A. Gavilanes.

Els Vanhoutte: Peripheral Neuropathy outcome measures; Standardisation (PeriNomS)

study part 2: Getting consensus. Supervisors: Prof.dr. C. Faber / Prof.dr. P. van Doorn;

Co-Supervisor: Dr. I. Merkies, Spaarne ziekenhuis Hoofddorp.

Mayienne Bakkers: Small fibers, big troubles; diagnosis and implications of small fiber 
neuropathy. Supervisors: Prof.dr. C. Faber / Prof.dr. M. de Baets; Co-Supervisor: Dr. I. Merkies, Spaarne ziekenhuis Hoofddorp.

Ingrid Kramer: Zooming into the micro-level of experience: An approach for understanding and treating psychopathology. Supervisor: Prof.dr. J. van Os; Co-Supervisors: Dr. M. Wichers, UMC Groningen / Dr. C. Simons.

Esther Bouman: Risks and Benefits of Regional Anesthesia in the Perioperative Setting. Supervisors: Prof.dr. M. van Kleef/Prof.dr. M. Marcus, HMC, Qatar/Prof.dr. E. Joosten; Co-Supervisor: Dr. H. Gramke.

Mark Janssen: Selective stimulation of the subthalamic nucleus in Parkinson's disease; dream or near future. Supervisors: Prof.dr. Y. Temel / Prof.dr. V. Visser-Vandewalle, Keulen / Prof.dr. A. Benazzouz, Bordeax, France.

Reina de Kinderen: Health Technology Assessment in Epilepsy; economic evaluations and preference studies. Supervisors: Prof.dr. S. Evers / Prof.dr. A. Aldenkamp; CoSupervisor: Dr. H. Majoie / Dr. D. Postulart, GGZ O-Brabant.

Saskia Ebus: Interictal epileptiform activity as a marker for clinical outcome.

Supervisors: Prof.dr. A. Aldenkamp / Prof.dr. J. Arends, TUE / Prof.dr. P. Boon, Universiteit Gent, België.

Inge Knuts: Experimental and clinical studies into determinants of panic severity. Supervisor: Prof.dr. I. Myin-Germeys; Co-Supervisor: Dr. K. Schruers; Influencing panic. Nienke Tielemans: Proactive coping post stroke: The Restored4Stroke Self-Management study. Supervisors: Prof.dr. C. van Heugten / Prof.dr. J. Visser-Meily, UMC Utrecht; Co-Supervisor: Dr. V. Schepers, UMC Utrecht.

Tom van Zundert: Improvements Towards Safer Extraglottic Airway Devices. Supervisors: Prof.dr. A.E.M. Marcus / Prof.dr. W. Buhre / Prof.dr. J.R. Brimacombe, Queensland, Australia / Prof.dr. C.A. Hagberg.

Tijmen van Assen: Anterior Cutaneous Nerve Entrapment Syndrome Epidemiology and surgical management. Supervisors: Prof.dr. G.L. Beets / Prof.dr. M. van Kleef / Dr. R.M.H. Roumen / Dr. M.R.M. Scheltinga, MMC Veldhoven.

Rohit Shetty: Understanding the Clinical, Immunological and Genetic Molecular Mechanisms of Keratoconus. Supervisors: Prof.dr. R.M.M.A. Nuijts / Prof.dr. C.A.B. Webers.

Christine van der Leeuw: Blood, bones and brains; peripheral biological endophenotypes and their structural cerebral correlates in psychotic disorder. Supervisor: Prof.dr. J. van Os; Co-supervisor: Dr. M. Marcelis.

Sanne Peeters: The Idle Mind Never Rests; functional brain connectivity across the psychosis continuum. Supervisor: Prof.dr. J. van Os; Co-supervisor: dr. M. Marcelis. Nick van Goethem: $\alpha 7$ nicotinic acetylcholine receptors and memory processes: 
mechanistic and behavioral studies. Supervisor: Prof.dr. H.W.M. Steinbusch; Cosupervisor: Dr. J. Prickaerts.

Nicole Leibold: A Breath of fear; a translational approach into the mechanisms of panic. Supervisor: Prof.dr. H.W.M. Steinbusch; Co-supervisors: Dr. K.R.J. Schruers / Dr. D.L.A. van den Hove.

Renske Hamel: The course of mild cognitive impairment and the role of comorbidity. Supervisor: Prof.dr. F.R.J. Verhey; Co-supervisors: Dr. I.H.G.B. Ramakers / Dr. P.J. Visser.

Lucia Speth: Effects of botulinum toxin $A$ injections and bimanual task-oriented therapy on hand functions and bimanual activities in unilateral Cerebral Palsy. Supervisors: Prof.dr. J. Vles; Prof.dr. R. Smeets; Co-supervisor: Dr. Y. Janssen-Potten, Adelante Hoensbroek.

Yuan Tian: The effects of Lutein on the inflammatory pathways in age-related macular degeneration(AMD). Supervisors: Prof.dr. C. Webers; Prof.dr. A. Kijlstra, WUR; Cosupervisor: Dr. M. Spreeuwenberg; Dr. H. Tange.

Peggy Spauwen: Cognition and Type 2 diabetes; the interplay of risk factors.

Supervisors: Prof.dr. F. Verhey; Prof.dr. C. Stehouwer; Co-supervisor: Dr. M. van Boxtel Marc Hilhorst: Crescentic glomerulonephritis in ANCA associated vasculitis.

Supervisors: Prof.dr. J. Cohen-Tervaert; Co-supervisor: Dr. P. van Paassen.

Martin Gevonden: The odd one out: exploring the nature of the association between minority status and psychosis. Supervisors: Prof.dr. J-P. Selten; Prof.dr. J. Booij, Uva; Prof.dr. I. Myin-Germeys.

Bart Biallosterski: Structural and functional aspects of sensory-motor Interaction in the urinary bladder. Supervisors: Prof.dr. Ph. Van Kerrebroeck; Prof.dr. S. De Wachter, UvAntwerpen; Co-supervisors: Dr. G. van Koeveringe; Dr. M. Rahnama'i.

Alexandra König: The use of information and communication technologies (ICT) for the assessment of patients with Alzheimer's Disease and related disorders.

Supervisors: prof.dr. F. Verhey; prof.dr. Ph. Robert, Nice, Fr; Co-supervisors: dr. P. Aalten; dr. R. David, Nice. Fr.

Michelene Chenault: Assessing Readiness for Hearing Rehabilitation.

Supervisors: prof.dr. M.P.F. Berger; prof.dr. B. Kremer; Co-supervisor: dr. L.J.C. Anteunis.

Anand Vinekar: Retinopathy of Prematurity. Recent advances in tele-medicine screening, risk factors and spectral domain optical coherence tomography imaging. Supervisor: prof.dr. C.A.B. Webers; Co-supervisor: dr. N.J. Bauer. 
Fleur van Dooren: Diabetes and Depression: exploring the Interface between

Pathophysiological and Psychological factors. Supervisors: prof.dr. F.R.J. Verhey; prof.dr. J.K.L. Denollet, UvT; prof.dr. F. Pouwer, UvT; Co-supervisor: dr. M.T. Schram. Gabriëlla Pons van Dijk: Taekwondo and physical fitness components in middle-aged healthy volunteers; the Sekwondo study. Supervisors: prof.dr. J. Lodder; prof.dr. H. Kingma; Co-supervisor: dr. A.F. Lenssen.

Yara Pujol López: Development and psychoneuroimmunological mechanisms in depression. Supervisor: prof.dr. H.W.M. Steinbusch; Co-supervisors: Dr. G. Kenis; Dr. D. van den Hove; Dr. Aye Mu Myint, München.

Romina Gentier: UBB ${ }^{+1}$; an important switch in the onset of Alzheimer's disease.

Supervisors: Prof. H. Steinbusch; Prof. D. Hopkins; Co-supervisor: Dr. F. van Leeuwen. Sanne Smeets: Insights into insight: studies on awareness of deficits after acquired brain injury.

Supervisor: Prof. C. van Heugten; Prof. R. Ponds; Co-supervisor: Dr. I. Winkens Kim Beerhorst: Bone disease in chronic epilepsy: fit for a fracture.

Supervisor: Prof. A. Aldenkamp; Prof. R. van Oostenbrugge; Co-supervisor: Dr. P. Verschuure.

Alex Zwanenburg: Cerebral and cardiac signal monitoring in fetal sheep with hypoxicischemic encephalopathy. Supervisor: Prof. T. Delhaas; Prof. B. Kramer; Cosupervisors: Dr. T. Wolfs; Dr. P. Andriessen, MMC.

Ismail Sinan Guloksuz: Biological mechanisms of environmental stressors in psychiatry. Supervisor: Prof. J. van Os; Co-supervisors: Dr. B. Rutten; Dr. M. Drukker.

Seyed Ehsan Pishva MD: Environmental Epigenetics in mental health and illness.

Supervisor: Prof.dr. J. van Os; Co-supervisors: Dr. B.P.F. Rutten; Dr. G. Kenis.

Ankie Hamaekers: Rescue ventilation using expiratory ventilation assistance; innovating while clutching at straws. Supervisors: Prof.dr. W.F. Buhre; Prof.dr. M. van Kleef.

Rens Evers. 22q11.2 deletion syndrome: intelligence, psychopathology and neurochemistry at adult age. Supervisors: Prof.dr. L.M.G. Curfs; Prof.dr. T. v. Amelsvoort.

Sarah-Anna Hescham. Novel insights towards memory restoration.

Supervisor: Prof.dr. Y. Temel; Co-supervisor: Dr. A. Blokland; Dr. A. Jahanshahi. João P. da Costa Alvares Viegas Nunes. Insulin receptor sensitization improves affective pathology in various mouse models. Supervisor: Prof.dr. H.W.M. Steinbusch; Co-supervisors: Dr. K-P. Lesch; Dr. T. Strekalova; Dr.B.H. Cline, Oxford. Yanny Ying-Yee Cheng. Clinical Outcomes After Innovative Lamellar Corneal Transplantation Surgery. Supervisor: Prof. dr. R.M.M.A. Nuijts; Co-supervisor: Dr. J.S.A.G. Schouten. 